BNL-96973-2009-IR

$\mathrm{C}-\mathrm{A} / \mathrm{AP} / \# 452$

Feb. 2012

\title{
Spin tracking simulations in AGS based on ray-tracing methods - bare lattice, no snakes -
}

\author{
F. Meot, L. Ahrens, J. Glenn, H. Huang, \\ A. Luccio, W. W. MacKay, T. Roser, N. Tsoupas
}

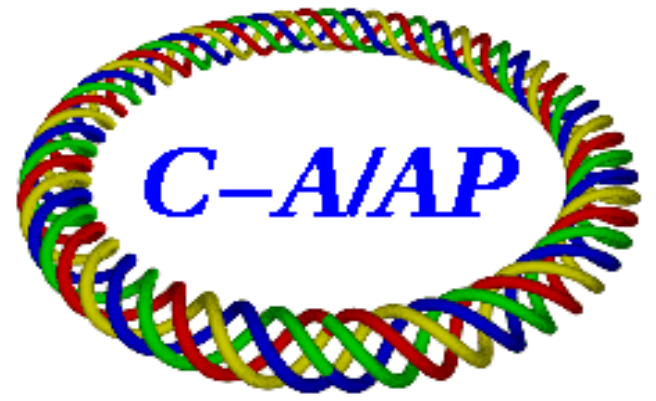

Collider-Accelerator Department Brookhaven National Laboratory

Upton, NY 11973

Notice: This document has been authorized by employees of Brookhaven Science Associates, LLC under Contract No. DE-AC02-98CH10886 with the U.S. Department of En ergy. The United States Government retains a nonexclusive, paid-up, irrevocable, world-wide license to publish or reproduce the published form of this document, or allow others to do so, for United States Government purposes. 
F. Méot, BNL, Sept. 2009

C-AD/AP/452

\title{
Spin tracking simulations in AGS based on ray-tracing methods - bare lattice, no snakes -
}

A work performed at BNL in September and October 2009, in collaboration with

L. Ahrens, J. Glenn, H. Huang, A. Luccio, W. W. MacKay, T. Roser, N. Tsoupas

\begin{abstract}
This Note reports on the first simulations of and spin dynamics in the AGS using the ray-tracing code Zgoubi.

It includes lattice analysis, comparisons with MAD, DA tracking, numerical calculation of depolarizing resonance strengths and comparisons with analytical models, etc. It also includes details on the setting-up of Zgoubi input data files and on the various numerical methods of concern in and available from Zgoubi.

This work has been followed by further spin dynamics studies in presence of the AGS helical snakes, see Note CAD/AP/453.
\end{abstract}




\section{Contents}

1 Introduction $\quad 4$

2 Preliminary data, working hypothesis $\quad 4$

2.1 Lattice . . . . . . . . . . . . . . . . . . . . . . . . . . 4

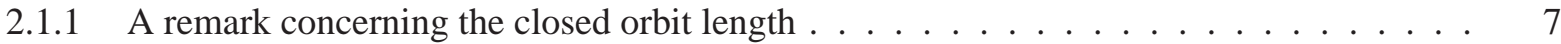

2.2 DA. Long term tracking . . . . . . . . . . . . . . . . . . . . . . . 7

2.2.1 Maximum stable amplitudes . . . . . . . . . . . . . . . . . . 7

2.2 .2 Dynamic aperture . . . . . . . . . . . . . . . . . . . 7

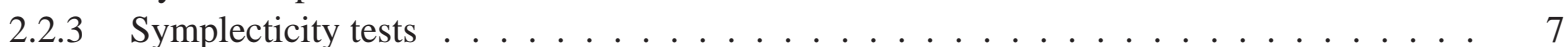

2.3 Quantities and formulas used in these spin tracking studies . . . . . . . . . . . . . 10

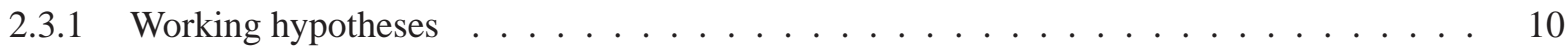

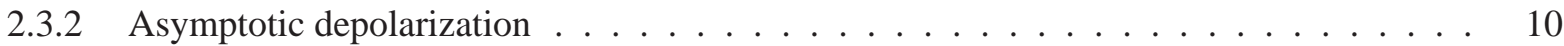

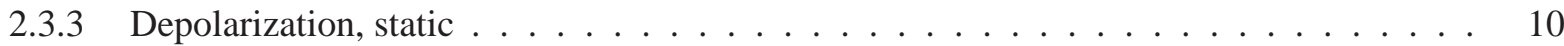

2.3.4 Weak resonance, Fresnel integral approximation . . . . . . . . . . . . . . . 11

2.3.5 Resonance strength, theoretical . . . . . . . . . . . . . . . . . . 11

3 Inventory of spin resonances, including spin tracking results 12

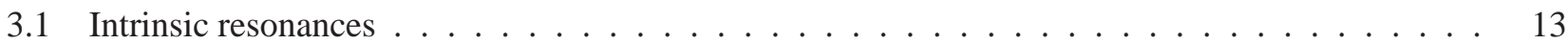

3.2 Imperfection resonances $\ldots \ldots \ldots \ldots \ldots \ldots \ldots \ldots$

4 Tracking through resonances $\quad 17$

4.1 Intrinsic resonances $\ldots \ldots \ldots \ldots \ldots \ldots \ldots \ldots \ldots$

$4.1 .1 \quad \gamma G=\nu_{z}(3.648013 \mathrm{GeV}) \ldots \ldots \ldots \ldots \ldots \ldots \ldots \ldots$

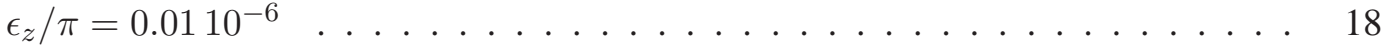

$\epsilon_{z} / \pi=0.0510^{-6} \ldots \ldots \ldots \ldots \ldots \ldots$

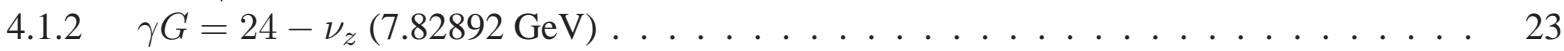

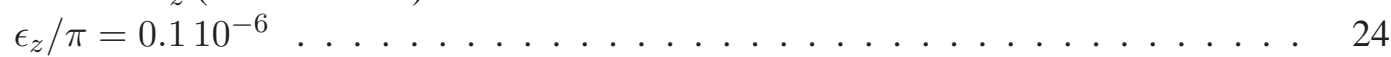

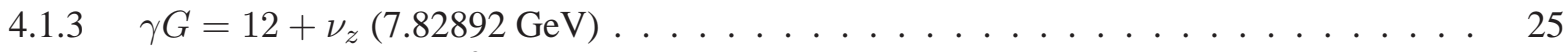

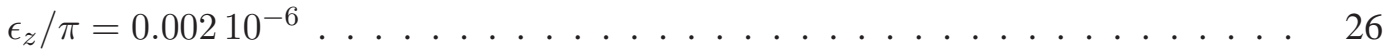

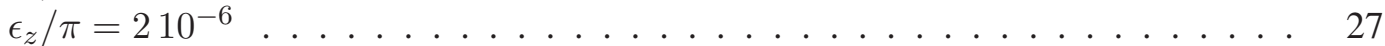

$4.1 .4 \gamma G=23+\nu_{z}(15.68487 \mathrm{GeV}) \ldots \ldots \ldots \ldots \ldots \ldots \ldots \ldots$

$4.1 .5 \gamma G=24+\nu_{z}(16.20822 \mathrm{GeV}) \ldots \ldots \ldots \ldots \ldots \ldots$

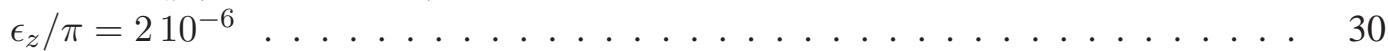

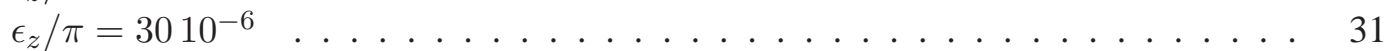

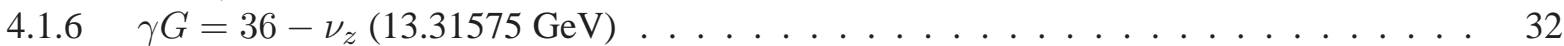

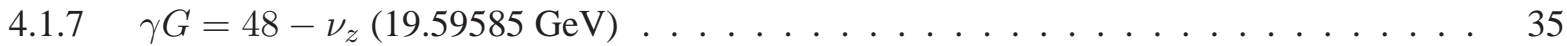

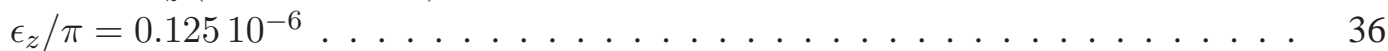

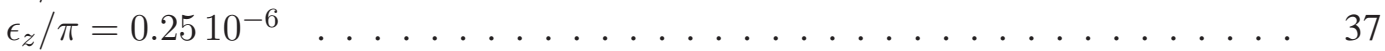

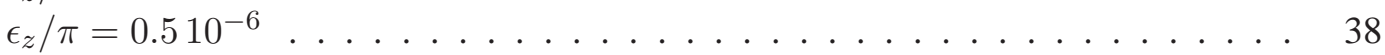

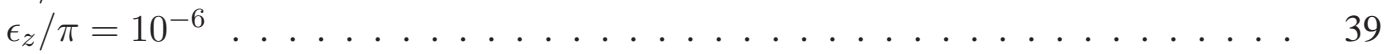

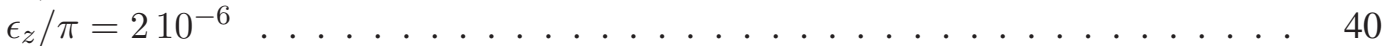

4.1.8 $\gamma G=48-\nu_{z}$, bare lattice (all bends' sextu off, all quads off) $\ldots \ldots \ldots \ldots$. . . . . 41

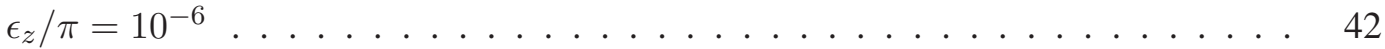

4.1.9 $\gamma G=36+\nu_{z}(22.48832 \mathrm{GeV}) \ldots \ldots \ldots \ldots \ldots \ldots$

$\epsilon_{z} / \pi=0.00210^{-6} \ldots \ldots \ldots \ldots \ldots \ldots \ldots \ldots$

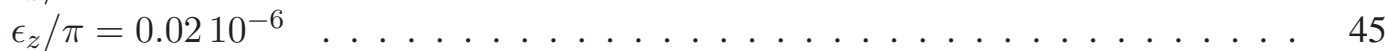

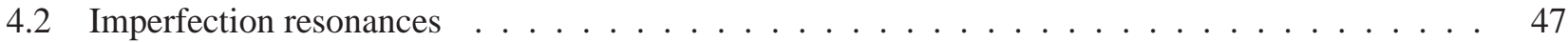

$4.2 .1 \quad \gamma G=9(3.77180 \mathrm{GeV}) \ldots \ldots \ldots \ldots \ldots \ldots \ldots \ldots$

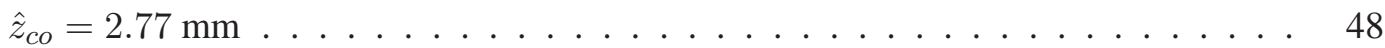

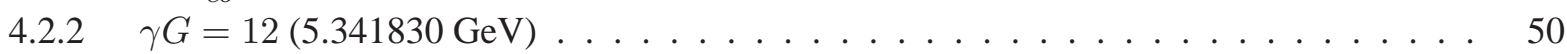

$4.2 .3 \gamma G=13(5.865171 \mathrm{GeV}) \ldots \ldots \ldots \ldots \ldots \ldots$ 
$4.2 .4 \quad \gamma G=23(11.09859 \mathrm{GeV}) \ldots \ldots \ldots \ldots \ldots \ldots \ldots \ldots$

$4.2 .5 \gamma G=27(13.19196 \mathrm{GeV}) \ldots \ldots \ldots \ldots \ldots \ldots \ldots$

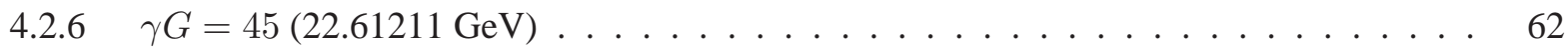

5 Static neighboring of resonances $\quad 65$

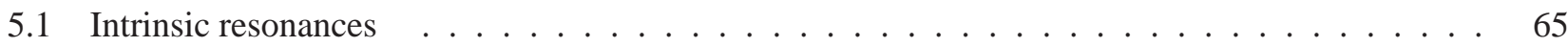

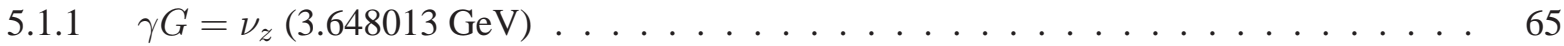

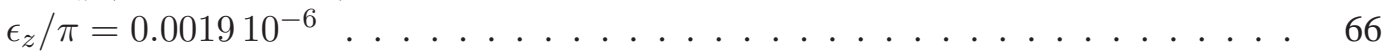

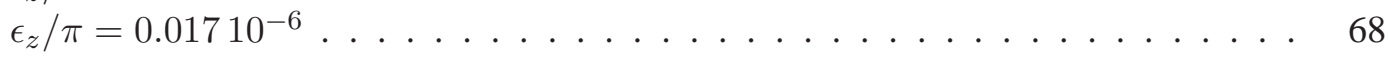

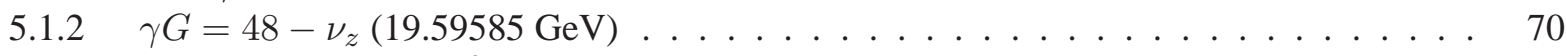

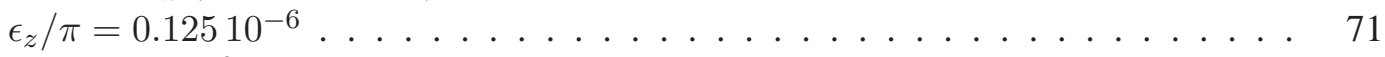

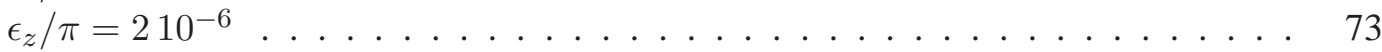

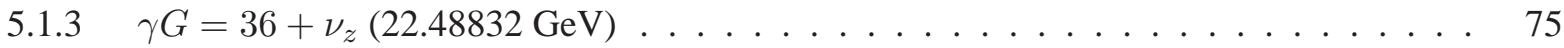

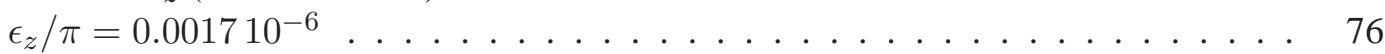

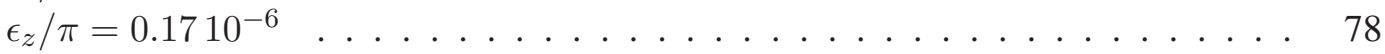

$\begin{array}{llr}6 & \text { Fresnel integrals approximation of weak resonances } & \mathbf{8 0}\end{array}$

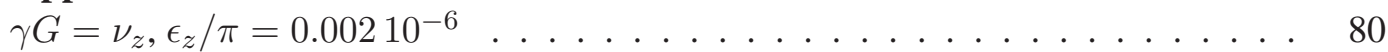

$\gamma G=\nu_{z}, \epsilon_{z} / \pi=0.0110^{-6} \ldots \ldots \ldots \ldots \ldots \ldots$

$\gamma G=36+\nu_{z}, \epsilon_{z} / \pi=0.000110^{-6} \ldots \ldots \ldots \ldots \ldots \ldots \ldots$

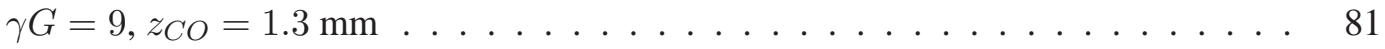

$\gamma G=45, z_{C O}=0.028 \mathrm{~mm} \ldots \ldots \ldots \ldots 1$

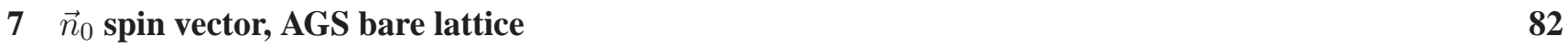

$\begin{array}{ll}\text { Appendix } & 84\end{array}$

$\begin{array}{lr}\text { A MAD files } & 84\end{array}$

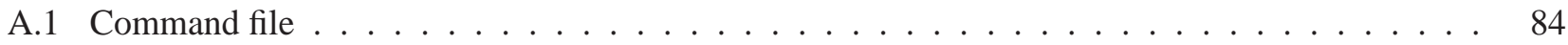

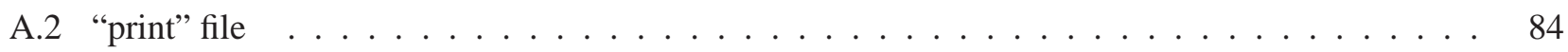

B Zgoubi data file specimen $\quad 85$

B.1 1-turn first order mapping calculation $\ldots \ldots \ldots \ldots \ldots \ldots$

B.2 Zgoubi files, $\vec{n}_{0}$ vector search using FIT $\ldots \ldots \ldots \ldots \ldots$

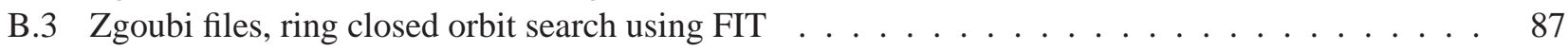




\section{Introduction}

Simulations of crossing and neighboring of spin resonances in AGS ring, bare lattice, without snake, have been performed, in order to assess the capabilities of Zgoubi in that matter, and are reported here. This yields a rather long document. The two main reasons for that are, on the one hand the desire of an extended investigation of the energy span, and on the other hand the fact that in doing so, it has been discovered that the agreement with DEPOL values for the resonance strengths is not good for weak resonances, thus entailing even more systematic exploration of the effects.

As to this disagreement in the resonance strengths, whether the weak reasoning is in Zgoubi or in DEPOL needs be determined, this is being worked at.

Section 2 details the working hypothesis : AGS lattice data, formulae used for deriving various resonance related quantities from the ray-tracing based "numerical experiments", etc.

Section 3 gives inventories of the intrinsic and imperfection resonances together with the strengths derived from the ray-tracing.

Section 4 and Section 4.2 give the details of the numerical simulations of resonance crossing, including behavior of various quantities (close orbit, synchrotron motion, etc.) aimed at controlling that the conditions of particle and spin motions are correct.

In a similar manner Section 5 gives the details of the numerical simulations of spin motion in the static case : fixed energy in the neighboring of the resonance.

Section 7 shows the computation of the $\vec{n}$ vector in the AGS lattice and tuning considered.

Many details on the numerical conditions as data files etc. are given in the Appendix Section, pages A and sqs.

\section{Preliminary data, working hypothesis}

This section details the working hypothesis, from both viewpoints of lattice data and of ray-tracing basic dynamics outputs.

\subsection{Lattice}

Tab. 1 displays the general optical parameters as obtained from MAD8, various input/output files of concern are reproduced in App. A.

Zgoubi optics file is translated from MAD8 "survey" output (a translator is available), parameter values so obtained are given in the middle col. ("Brute from MAD8) in Tab. 1 for comparison with MAD ones. A typical "zgoubi.dat" input file is displayed in App. B.1.

Note the $\approx 3.2 \mathrm{~cm}$ difference in orbit length (see below) and the $\approx 9 \%$ difference in $D_{x}$, to be explained. 
Table 1: AGS parameters. The middle column "Brute from MAD8" gives the working conditions in the numerical experiments reported in the following sections. The right most column shows how some parameter have to evolve so to obtain zero closed orbit at main bend ends.

\begin{tabular}{|c|c|c|c|c|}
\hline \multirow[b]{3}{*}{ Reference momentum } & \multirow[b]{3}{*}{ (relative) } & \multirow[t]{2}{*}{ MAD8 } & \multicolumn{2}{|c|}{$\begin{array}{c}\text { Ray-tracing }{ }^{1} \\
\text { (Hard edge) }\end{array}$} \\
\hline & & & Brute from MAD8 & Adjusted c.o. length ${ }^{5}$ \\
\hline & & 1 & 1 & 1.00280 \\
\hline Orbit length $^{4}$ & $(\mathrm{~m})$ & 807.07564 & 807.04378 & 807.07564 \\
\hline Perimeter of polygon ${ }^{(6)}$ & (m) & & 807.06007 & \\
\hline Qx, Qy & & $8.71060,8.76438$ & {$[8] .71195,[8] .76346^{2}$} & {$[8] .65346,[8] .76816$} \\
\hline Q’x, Q'y & & $-22.7340,1.7343$ & $-20.9864,1.7943$ & \\
\hline$\alpha, \sqrt{1 / \alpha}$ & & $0.01401,8.44965$ & $0.01400,8.45102$ & \\
\hline Periodic functions at "Begin AGS": & & & & \\
\hline$\beta_{x}, \beta_{y}$ & $(\mathrm{~m})$ & $19.785,11.701$ & $19.8432,11.6751$ & \\
\hline$\alpha_{x}, \alpha_{y}$ & & $-1.585,1.037$ & $-1.588,1.033$ & \\
\hline$D_{x}, D_{x}^{\prime}$ & $(\mathrm{m},-)$ & $2.211,0.154$ & $2.034,0.144$ & \\
\hline closed orbit, $x_{c o}, x_{c o}^{\prime}$ & $\mathrm{mm}, \mathrm{mrad}$ & 0,0 & $-6.43-0.50^{1,3}$ & $\approx 0, \approx 0$ \\
\hline
\end{tabular}

${ }^{1}$ Combined function dipoles are simulated using straight axis multipoles and $d^{n} B / d x^{n}$ gradients, the "MULTIPOL" keyword, whereas MAD uses SBEND.

${ }^{2}$ Obtained from either coordinate interpolation or multiturn Fourier analysis.

${ }^{3}$ The c.o. is induced by straight axis dipole and multipole components.

${ }^{4}$ Note the substantial difference in orbit lengths - origin to be determined.

${ }^{5}$ Closed orbit adjusted using FIT procedure, zgoubi.dat data in App. B.3.

${ }^{4}$ Polygon is comprised of the axis of optical elements, all straight.

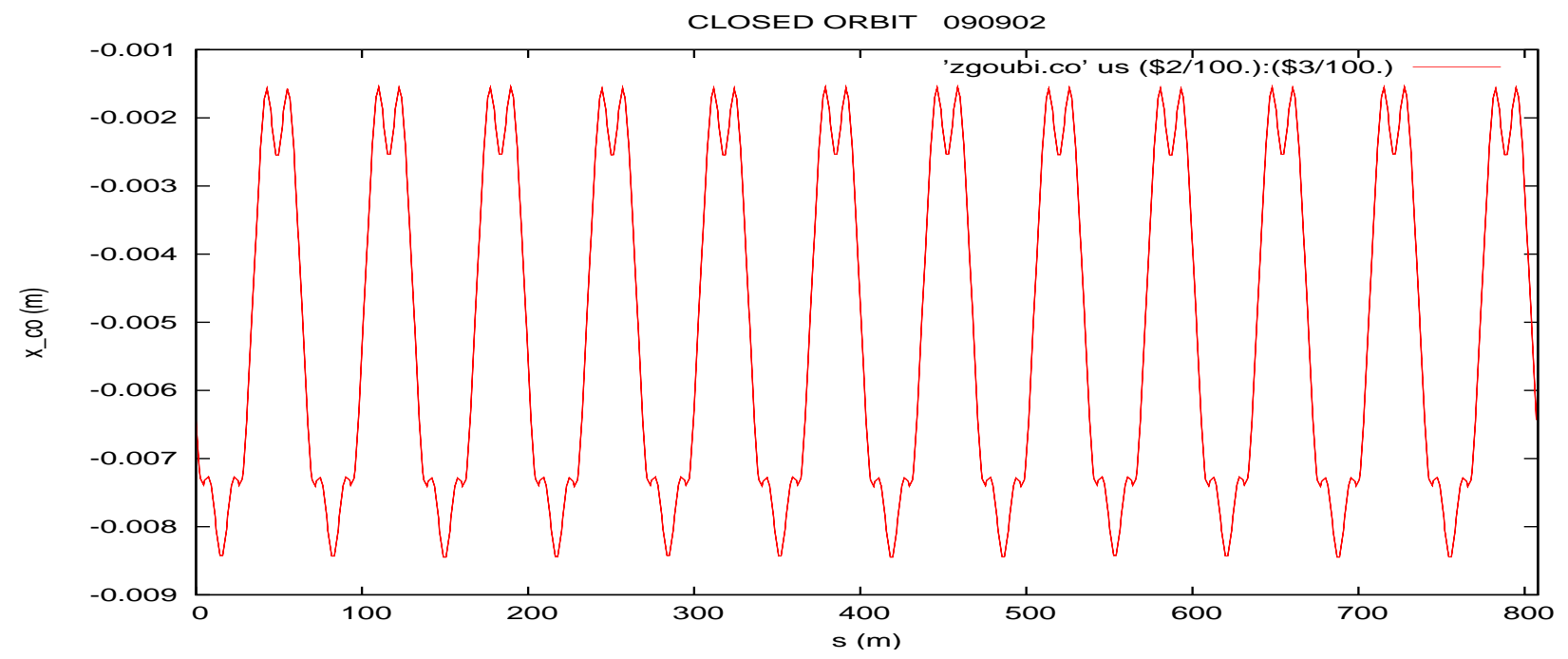

Figure 1: Closed orbit, induced by straight axis in bends (all hard-edge model). 
A superimposition of MAD/TWISS and Zgoubi outputs :

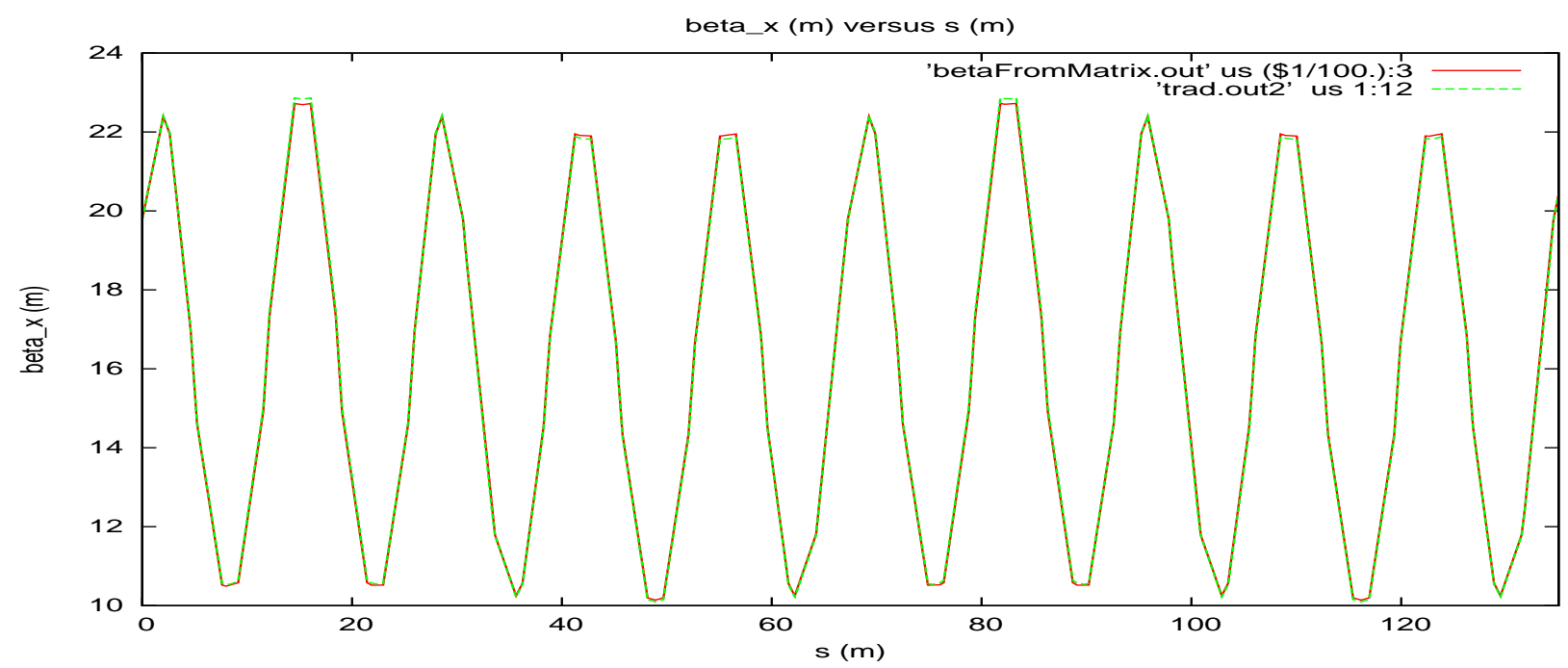

Figure 2: $\beta_{x}$ over the first two superperiods.

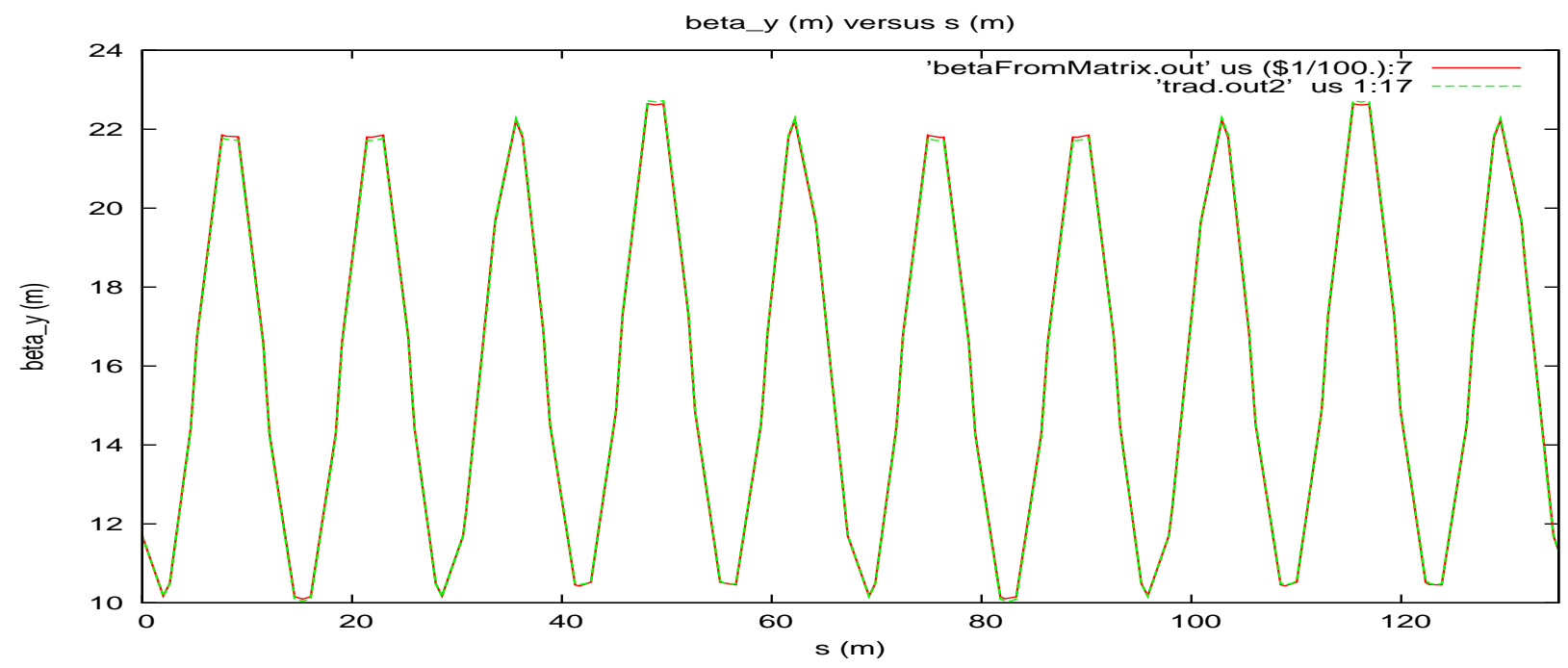

Figure 3: $\beta_{y}$ over the first two superperiods.

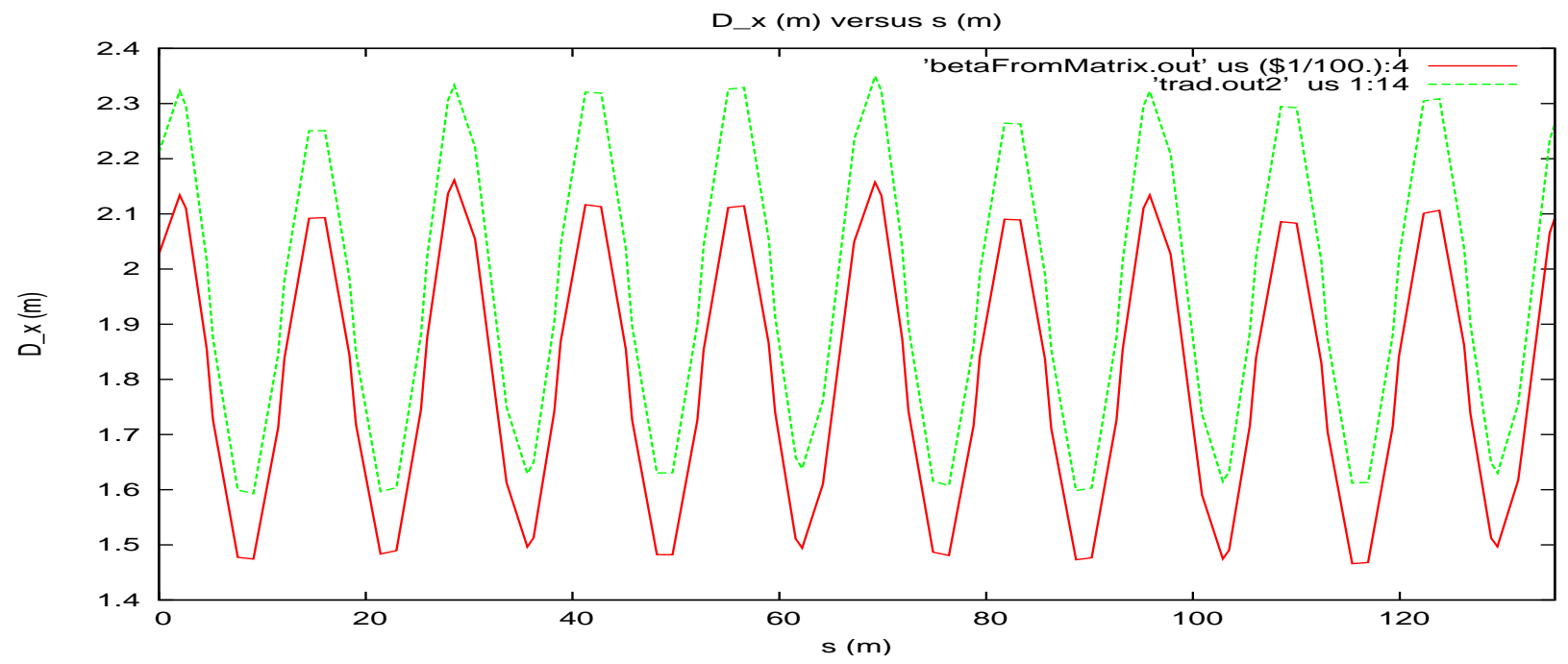

Figure 4: $D_{x}$ over the first two superperiods. 


\subsubsection{A remark concerning the closed orbit length}

The problem is the following : brute translation from MAD8 yields $3.2 \mathrm{~cm}$ shorter on-momentum closed orbit length from zgoubi (807.04378 m/zgoubi compared to 807.075641/MAD8, see cols. 1, 2 in Tab. 1.

Getting identical lengths can be achieved by changing the reference momentum, according to

$$
\Delta \mathcal{L} / L=\alpha \delta p / p
$$

$\alpha=0.01400$, an increase of $\Delta \mathcal{L} / L=(807.075641-807.04378) / 807.04378$ (Tab. 1), indicate that increasing the reference momentum in zgoubi by $d p / p \approx 2.810^{-3}$ is required.

However the closed orbit needs be found again for that new reference momentum value, so we use the FIT procedure to take care of both operations. The result in zgoubi.res is reproduced in App. B.3. (Note that, would tunes be matched using possible main bend windings, tune constraints could be added in the FIT.)

Tunes then change due to chromaticity mostly, according to

$\Delta \nu_{x}=\xi_{x} \delta p / p \approx-0.059 \Rightarrow \nu_{x} \approx 8.653$

$\Delta \nu_{y}=\xi_{y} \delta p / p \approx \Rightarrow \nu_{y} \approx 8.769$

this is confirmed by the ray-tracing values (rightmost column in Tab. 1).

\subsection{DA. Long term tracking}

Considering the importance of a good model of the ring dynamics if spin is to be tracked, we push further the investigation on the lattice we are working with, and of the ray-tracing outputs, by checking large excursion behavior, in terms of maximum stable amplitudes and DA. Long term tracking behavior is also tested.

\subsubsection{Maximum stable amplitudes}

Figs. 5, 6 show sample results of maximum stable amplitude tracking, 1000 turns.

It can be observed that the numerical integration in the horizontal case (Fig. 5) does not exhibit noticeable spiraling, an indication of correct symplectic behavior.

In the case of the vertical stability limit tracking (Fig. 6), coupling induces large horizontal motion, at the origin of spreading of the vertical invariant into a donut phase portrait ; the origin (e.g., width of non-linear coupling resonance, lack of tracking precision...) remains to be determined.

\subsubsection{Dynamic aperture}

Fig. 8 gives the dynamic aperture of the ring for $\delta p / p=0, \pm 1, \pm 2, \pm 3 \%$. These $(x, z)$ limits are obtained by scanning the $\mathrm{x}$-axis, step size $3 \mathrm{~cm}$, and looking, at each $x$-step, for the maximum vertical stable amplitude upon 1000-turn tracking (namely, doing what is illustrated in Fig. 6), with $2 \mathrm{~mm}$ precision on that $z$-limit. The operation is performed repeatedly for the various momenta.

\subsubsection{Symplecticity tests}

Long term tracking may be required in assessing depolarizing effects in AGS or RHIC. Fig. 9 displays some sample results, showing very good behavior. The integration step size is $\sim 1 \mathrm{~cm}$ in all optical elements.

In the case of RHIC, it the step size can be a little larger given that the dipoles are not combined function. It has been checked that $1.5 \mathrm{~cm}$ at top energy (where spin moves the fastest in the dipoles) does ensure in addition convergence of the solution of $\vec{S}^{\prime}=\vec{x} \vec{\omega}$. 
Spin has been tracked in AGS step by step as well during this $n \approx 510^{5}$-turn motion tracking. Zgoubi calculates independently the three components, $S_{x}, S_{y}, S_{z}$. It comes out that $|\vec{S}|^{2}=S_{x}^{2}+S_{y}^{2}+S_{z}^{2} \equiv 1$, from beginning to end. Similar behavior in RHIC at top energy with $1.5 \mathrm{~cm}$ step size.
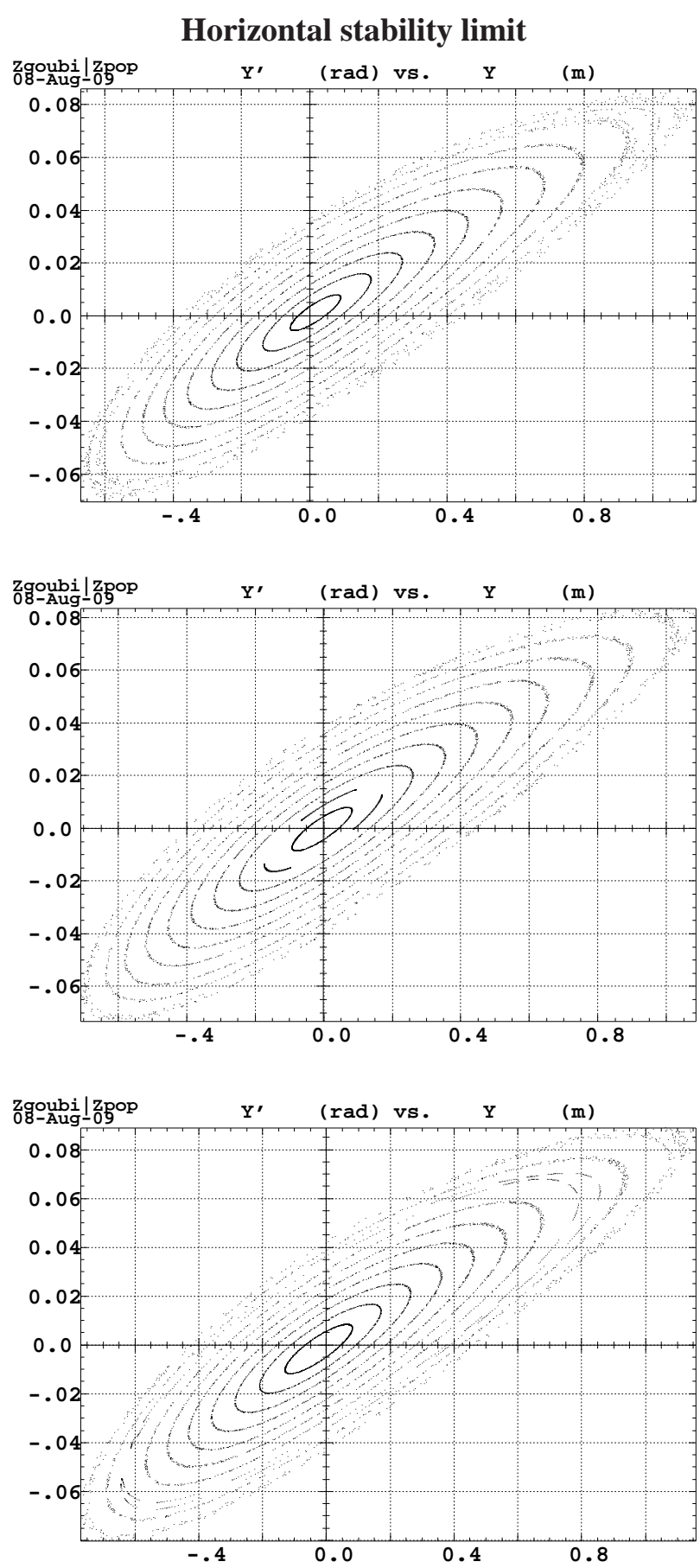

Figure 5: Maximum horizontal stable amplitude, 1000 turns in the ring, case of zero vertical emittance.

From top to bottom : $\delta p / p=+0.01,0,-0.01$. Hard edge optical elements.
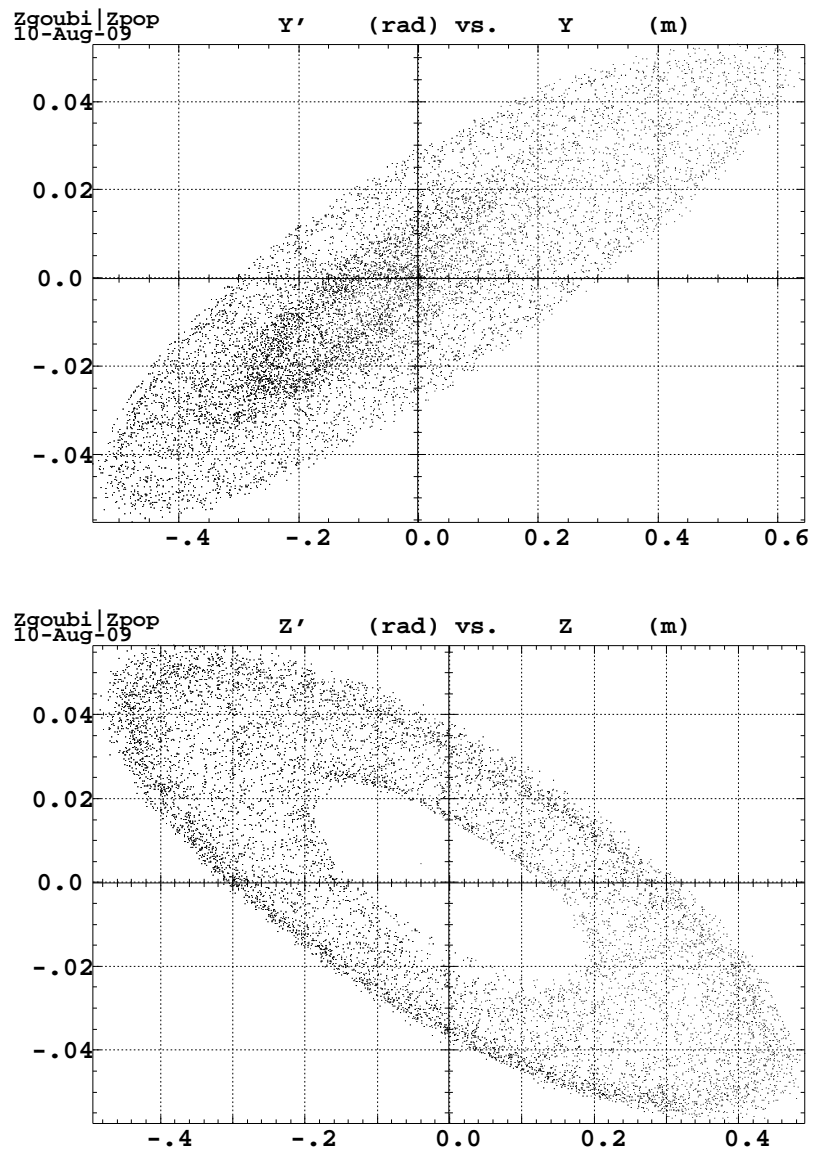

Figure 6: Maximum vertical stable amplitude, 1000 turns, showing $\mathrm{H}-\mathrm{V}$ emittance exchange, $\delta p / p=0$.

Top plot : coupling induced $x$-motion ; bottom plot : vertical donut at maximum stable amplitude.

Hard edge optical elements.
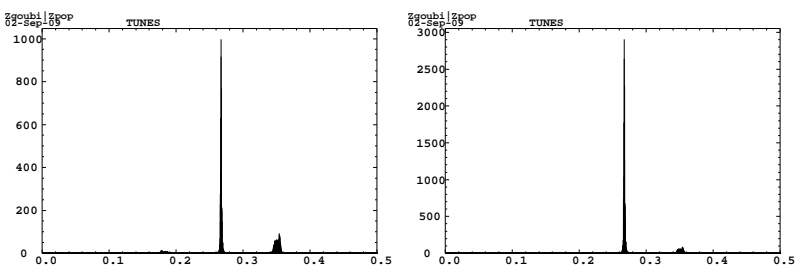

Figure 7: $x-x^{\prime}$ and $z-z^{\prime}$ spectra (left, right), exhibiting coupling. 


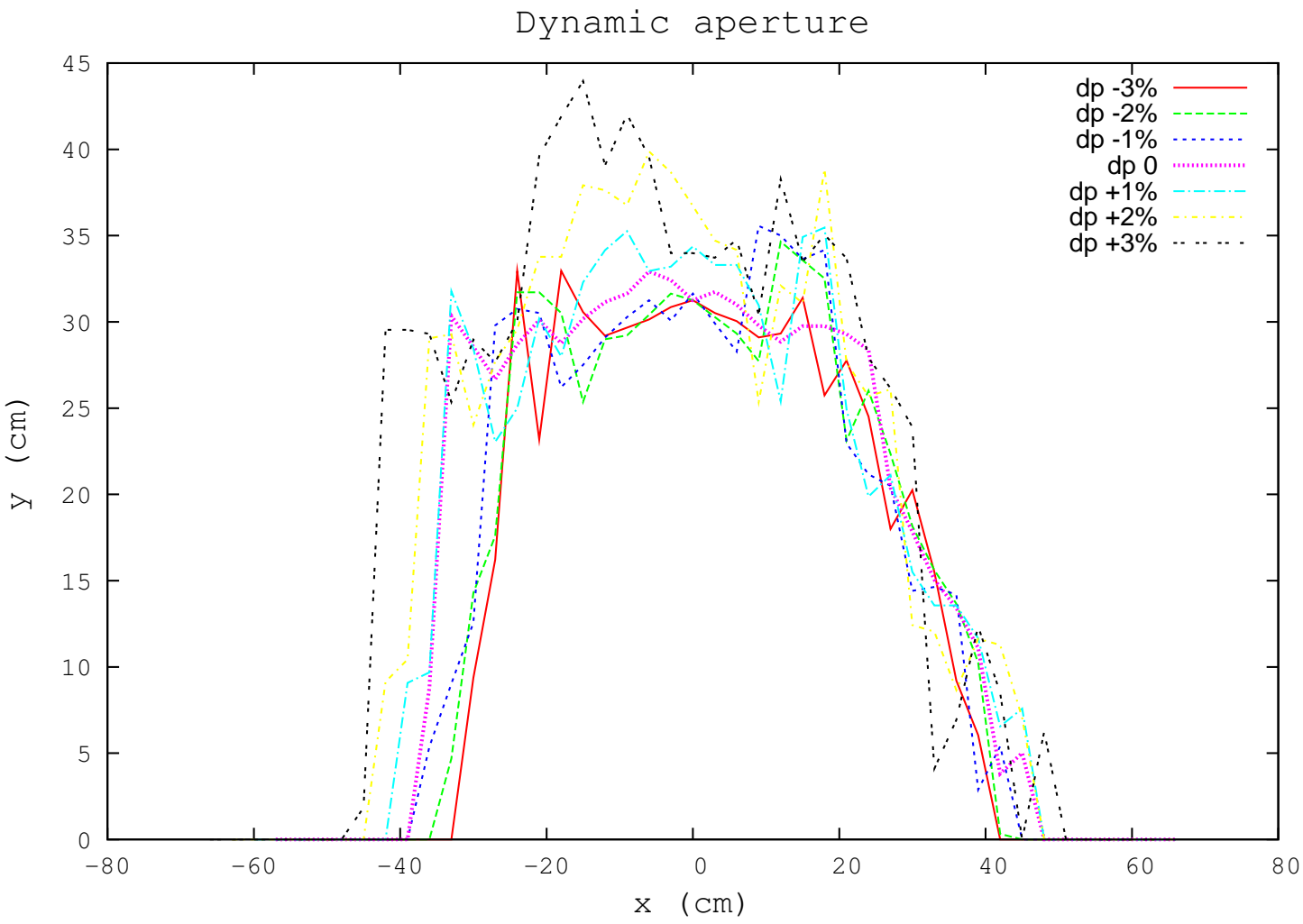

Figure 8: Dynamical apertures at $\delta p / p=0, \pm 1, \pm 2, \pm 3 \% .3 \mathrm{~cm}$ step in $x, 2 \mathrm{~mm}$ precision on 1000-turn maximum vertical stable amplitude.

Hard edge optical elements.
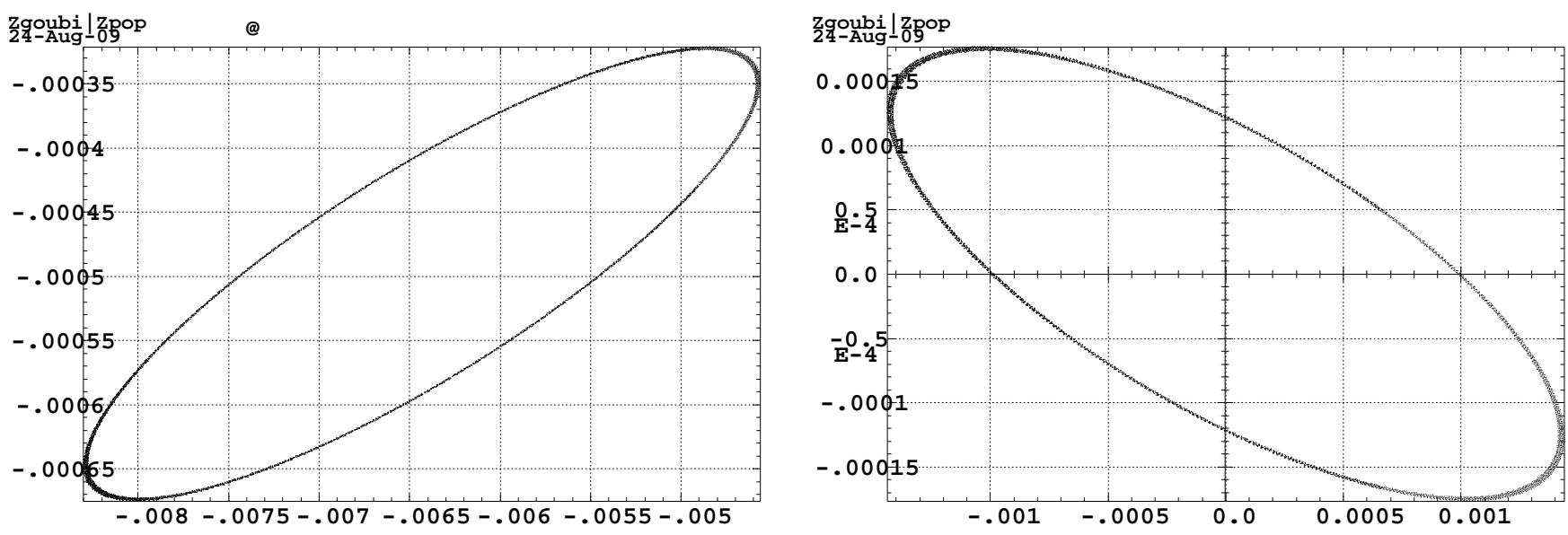

Figure 9: $410^{5}$-turn tracking for a particle launched on $\epsilon_{x} / \pi=\epsilon_{z} / \pi=0.35 \mathrm{~mm}$. mrad, at fixed rigidity, observed at "Begin AGS".

There is no substantial broadening of the invariants, nor visible spiraling.

Note : a matching of optical functions yields, $\beta_{x}=19.8 \mathrm{~m}, \alpha_{x}=-1.58, \beta_{z}=11.7 \mathrm{~m}, \alpha_{z}=1.03$, consistent with Tab. 1 data.

Hard edge optical elements. 


\subsection{Quantities and formulas used in these spin tracking studies}

Classical formulae which will be used in the "numerical experiments" to follow are recalled below, together with various numerical data values ${ }^{1}$.

\subsubsection{Working hypotheses}

The crossing speed writes

$$
\alpha=G \frac{d \gamma}{d \theta}=G \frac{1}{2 \pi} \frac{\Delta E}{M_{0}}
$$

with $M_{0}=938.27203 \mathrm{MeV}, G=1.7928474$. In the numerical simulations we take

$$
\begin{aligned}
& \hat{V}=290 \mathrm{kV} \\
& \phi_{s}=30 \text { degrees or } 150 \text { degrees }
\end{aligned}
$$

hence

$$
\Delta E=145 \mathrm{keV} / \mathrm{turn} \quad \text { and } \quad \alpha=4.409635610^{-5}
$$

Besides,

$$
\dot{B}=\Delta E /(2 \pi R \rho)=\Delta E /(\mathcal{C} \rho)
$$

The ring circumference is (Tab. 1)

$\mathcal{C}=807.04378 \mathrm{~m}$

whereas $1 / \rho=B / B \rho=0.01171255$ (see dipole component in 'SBEN' type of magnets, zgoubi.dat file in App. B.1). (Note : $\rho=85.378504$, packing factor $R / \rho=9.4525406$ ). This yields

$$
\dot{B}=2.104 \mathrm{~T} / \mathrm{s}
$$

\subsubsection{Asymptotic depolarization}

Crossing of an isolated resonance entails asymptotic depolarization given by the Froissard-Stora formula,

$$
\frac{p_{\text {final }}}{p_{\text {initial }}}=2 \exp \left(-A^{2}\right)-1=2 \exp \left(-\frac{\pi}{2} \frac{\left|J_{n}\right|^{2}}{\alpha}\right)-1
$$

\subsubsection{Depolarization, static}

Free oscillation of polarization vector $\vec{S}$ around arbitrary local precession vector $\vec{\omega}$, at fixed energy, starting with $\vec{S} \equiv \vec{S}_{z}$, satisfies

$$
\frac{(-)}{\sqrt{1-|C|^{2}}} \frac{d C}{d \theta}=J_{n} e^{j \Delta \theta} \text { with } \mathrm{C} \text { of the form } e^{j \mu \theta} \text { and } \rho=\text { constant yielding } \rho^{2}=\frac{1}{1+\Delta^{2} /\left|J_{n}\right|^{2}}
$$

with

$$
\begin{gathered}
\Delta=\text { distance to the resonance }=\gamma G-\left(n \times M-\nu_{z}\right) \\
\bar{S}_{z}^{2}=1-|\rho|^{2} \text { yields } \quad \bar{S}_{z}^{2}=\frac{1}{1+\left|J_{n}\right|^{2} / \Delta^{2}}
\end{gathered}
$$

with $\bar{S}_{z}$ the average value of $S_{z}$. This allows computing $\left|J_{n}\right|$ from the numerical value of $S_{z}$, following

$$
\left|J_{n}\right|^{2}=\Delta^{2} /\left(\frac{1}{\rho^{2}}-1\right)
$$

In particular,

$1 \%$ depolarization $\left(\bar{S}_{z}=0.99\right)$ corresponds to $\Delta=\gamma G-n=7\left|J_{n}\right|\left(\right.$ an energy band $\left.\pm \delta \gamma= \pm 7\left|J_{n}\right| / G\right)$

$86.6 \%$ depolarization $(\rho=0.5)$ corresponds to $\Delta=\gamma G-n=\sqrt{3}\left|J_{n}\right|$

\footnotetext{
${ }^{1}$ Ref. Gérard Leleux, Traversée des résonances de dépolarisation, unpublished, SATURNE, Saclay, 15 Février 1992.
} 


\subsubsection{Weak resonance, Fresnel integral approximation}

Reference : Gérard Leleux, Traversée des résonances de dépolarisation, unpublished, SATURNE, Saclay, 15 Février 1992.

Depolarization in the case of weak resonances (i.e., $p_{f} / p_{i} \approx 1$ ) is given by

- upstream of the resonance $(\theta<0):\left(p(\theta) / p_{i}\right)^{2}=1-\frac{\pi}{\alpha}\left|J_{n}\right|^{2}\left[\left(0.5-C\left(-\theta \sqrt{\frac{a}{\pi}}\right)\right)^{2}+\left(0.5-S\left(-\theta \sqrt{\frac{a}{\pi}}\right)\right)^{2}\right]$

- downstream of the resonance $(\theta>0):\left(p(\theta) / p_{i}\right)^{2}=1-\frac{\pi}{\alpha}\left|J_{n}\right|^{2}\left[\left(0.5+C\left(\theta \sqrt{\frac{a}{\pi}}\right)\right)^{2}+\left(0.5+S\left(\theta \sqrt{\frac{a}{\pi}}\right)\right)^{2}\right]$

where

$$
C(x)=\int_{0}^{x} \cos \left(\frac{\pi}{2} t^{2}\right) d t, \quad S(x)=\int_{0}^{x} \sin \left(\frac{\pi}{2} t^{2}\right) d t
$$

are the Fresnel integrals.

Note that

$$
p(\theta) / p_{i} \stackrel{\theta \rightarrow \infty}{\longrightarrow} 1-\frac{\pi}{\alpha}\left|J_{n}\right|^{2}
$$

i.e., Froissard-Stora formula (Eq. 2) taken to first order in $\left|J_{n}\right|^{2} / \alpha$. The approximation holds in the limit that higher order terms can be neglected, i.e. $\pi\left|J_{n}\right|^{2} / \alpha$ is small enough compared to 1 .

In Section 6, Zgoubi is tested against the weak-resonance approximation.

\subsubsection{Resonance strength, theoretical}

It can be computed from MAD type of output, this allows comparison with ray-tracing results. The following formulae hold.

Imperfection, thin lens approximation :

$$
J_{n}=\frac{1+\gamma G}{2 \pi} \Sigma_{\text {Qpoles }}\left\{\begin{array}{c}
\cos \left(\gamma G \alpha_{i}\right) \\
\sin \left(\gamma G \alpha_{i}\right)
\end{array}\right\}(K L)_{i} z_{c o, i}
$$

with $\alpha_{i}$ cumulated bend angle, $z_{c o, i}$ the closed orbit amplitude, at quadrupole $i$ with strength $(K L)_{i}$.

Intrinsic, thin lens approximation :

$$
J_{n}^{ \pm}=\frac{1+\gamma G}{4 \pi} \Sigma_{\text {Qpoles }}\left\{\begin{array}{c}
\cos \left(\gamma G \alpha_{i} \pm \psi_{i}\right) \\
\sin \left(\gamma G \alpha_{i} \pm \psi_{i}\right)
\end{array}\right\}(K L)_{i} \sqrt{\beta_{z, i} \epsilon_{z} / \pi}
$$

with

$$
\psi_{i}=\int_{0}^{\theta_{i}} \frac{d s}{\beta_{z}}, \quad{ }^{\prime}+^{\prime} \operatorname{sign} \text { for } \gamma G+\nu_{z}-n=0, \quad{ }^{\prime}-^{\prime} \operatorname{sign} \text { for } \gamma G-\nu_{z}-n=0
$$

and $(K L)_{i}$ strength of quadrupole $i$ located at $\theta_{i}$. 


\section{Inventory of spin resonances, including spin tracking results}

Two inventories of the intrinsic resonances are given in Tables 2, 3 respectively, below. They include the numerical value of the resonance strengths as obtained for the numerical ray-tracing, in both dynamic (crossing) and static cases. The difference between both (a factor 3-4) remains to be elucidated. 


\subsection{Intrinsic resonances}

Table 2: Intrinsic resonances. $\nu_{z}=8.76346, M=12$ superperiods. $A^{2}=\frac{\pi}{2} \frac{\left|J_{n}\right|^{2}}{\alpha}$.

Intrinsic resonances, systematic, $k M \pm \nu_{z}, M=12$, classed by energy.

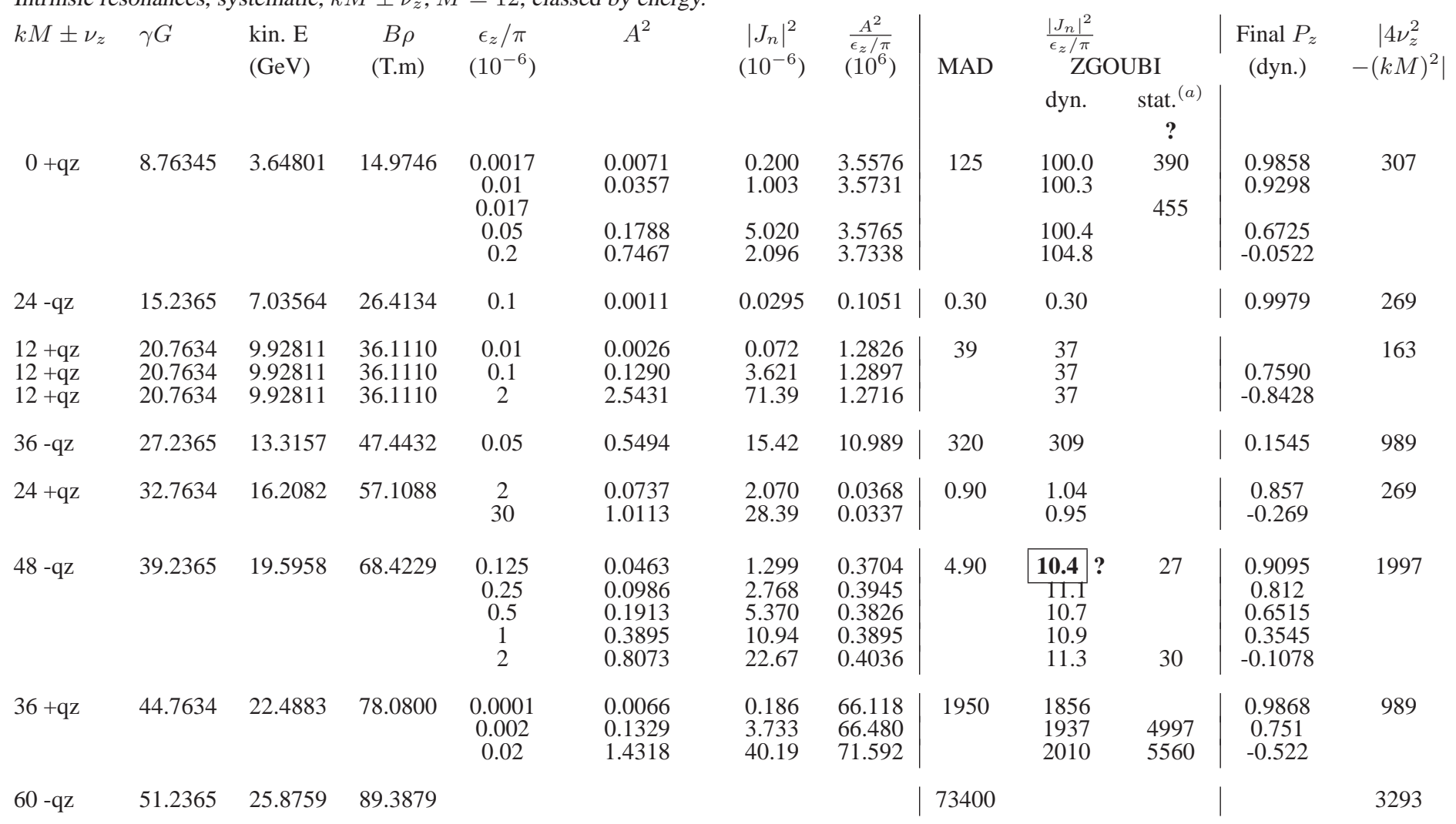

(a) Emittances in static case may slightly differ from that given in the ' $\epsilon_{z} / \pi$ ' column, exact values can be found in Sec. 5 .

\section{MAD, data obtained using :}

$J_{n}^{ \pm}=\frac{1+\gamma G}{4 \pi} \Sigma_{\text {Qpoles }}\left\{\begin{array}{c}\cos \left(\gamma G \alpha_{i} \pm \psi_{i}\right) \\ \sin \left(\gamma G \alpha_{i} \pm \psi_{i}\right)\end{array}\right\}(K L)_{i} \sqrt{\beta_{z, i} \epsilon_{z} / \pi}$
ZGOUBI , data obtained using :

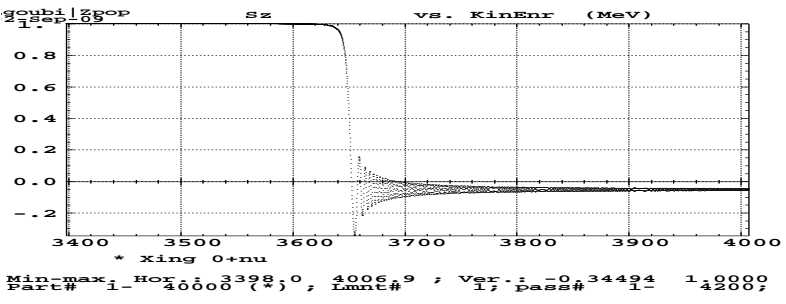

or

$\bar{S}_{z}=\sqrt{1 /\left(1+\left|J_{n}\right|^{2} / \Delta^{2}\right)}, \quad \Delta=\gamma G-\left(n \times M-\nu_{z}\right)$

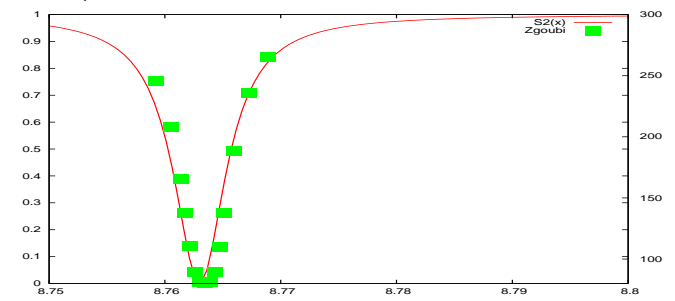




\subsection{Imperfection resonances}

A closed orbit is excited using a vertical kicker (DVCA02); the fourth column in Tab. 3 gives the maximum amplitude of the resulting closed orbit around AGS. 
Table 3: Closed orbit resonances.

\begin{tabular}{|c|c|c|c|c|c|c|c|c|c|}
\hline \multirow[t]{2}{*}{$\gamma G$} & \multirow{2}{*}{$\begin{array}{l}\text { kin. E } \\
(\mathrm{GeV})\end{array}$} & \multirow{2}{*}{$\begin{array}{c}B \rho \\
\text { (T.m) }\end{array}$} & \multirow{2}{*}{$\begin{array}{c}\hat{z} \\
(\mathrm{~mm})\end{array}$} & \multirow[t]{2}{*}{$A^{2}$} & \multirow{2}{*}{$\begin{array}{c}\left|J_{n}\right|^{2} \\
\left(10^{-5}\right)\end{array}$} & \multirow[t]{2}{*}{$A^{2} / \hat{z}^{2}$} & \multicolumn{2}{|c|}{$\left|J_{n}\right|^{2} / \hat{z}^{2}$} & \multirow[t]{2}{*}{ Final $P_{z}$} \\
\hline & & & & & & & MAD & $\begin{array}{c}\text { Zgoubi } \\
\text { dyn. }\end{array}$ & \\
\hline 5 & 1.678437 & 8.147990 & & & & & & & \\
\hline 6 & 2.201779 & 9.995557 & & & & & & & \\
\hline 7 & 2.725121 & 11.81217 & & & & & & & \\
\hline 8 & 3.248463 & 13.61023 & & & & & & & \\
\hline 9 & 3.771804 & 15.39624 & $\begin{array}{l}.139 \\
2.77\end{array}$ & 1.224737 & 3.4381555 & 159618 & 4.75 & 4.48 & -0.412 \\
\hline 10 & 4.295146 & 17.17396 & & & & & & & \\
\hline 11 & 4.818487 & 18.94571 & & & & & & & \\
\hline 12 & 5.341830 & 20.71305 & 13.84 & 0.6379532 & 1.7909011 & 3330 & .031 & 0.094 & 0.05674 \\
\hline 13 & 5.865171 & 22.47699 & 13.84 & 0.5469143 & 1.5353313 & 2855 & .019 & 0.080 & 0.15745 \\
\hline 14 & 6.388514 & 24.23830 & & & & & & & \\
\hline 15 & 6.911855 & 25.99749 & & & & & & & \\
\hline 16 & 7.435197 & 27.75499 & & & & & & & \\
\hline 17 & 7.958539 & 29.51107 & & & & & & & \\
\hline 18 & 8.481881 & 31.26600 & & & & & & & \\
\hline 19 & 9.005222 & 33.01994 & & & & & & & \\
\hline 20 & 9.528564 & 34.77305 & & & & & & & \\
\hline 21 & 10.05191 & 36.52545 & & & & & & & \\
\hline 22 & 10.57525 & 38.27723 & & & & & & & \\
\hline 23 & 11.09859 & 40.02848 & 13.84 & 1.734326 & 4.868705 & 905437 & .082 & 0.25 & -0.6468 \\
\hline 24 & 11.62193 & 41.77927 & & & & & & & \\
\hline 25 & 12.14527 & 43.52964 & & & & & & & \\
\hline 26 & 12.66861 & 45.27966 & & & & & & & \\
\hline 27 & 13.19196 & 47.02935 & 1.384 & 0.8763353 & 2.460102 & 457507 & 11.3 & 12.8 & -0.167355 \\
\hline 28 & 13.71530 & 48.77875 & & & & & & & \\
\hline 29 & 14.23864 & 50.52790 & & & & & & & \\
\hline 30 & 14.76198 & 52.27681 & & & & & & & \\
\hline 31 & 15.28532 & 54.02551 & & & & & & & \\
\hline 32 & 15.80867 & 55.77403 & & & & & & & \\
\hline 33 & 16.33201 & 57.52238 & & & & & & & \\
\hline 34 & 16.85535 & 59.27057 & & & & & & & \\
\hline 35 & 17.37869 & 61.01860 & & & & & & & \\
\hline 36 & 17.90203 & 62.76652 & & & & & & & \\
\hline 37 & 18.42537 & 64.51430 & & & & & & & \\
\hline 38 & 18.94872 & 66.26198 & & & & & & & \\
\hline 39 & 19.47206 & 68.00956 & & & & & & & \\
\hline 40 & 19.99540 & 69.75704 & & & & & & & \\
\hline 41 & 20.51874 & 71.50443 & & & & & & & \\
\hline 42 & 21.04208 & 73.25175 & & & & & & & \\
\hline 43 & 21.56543 & 74.99899 & & & & & & & \\
\hline 44 & 22.08877 & 76.74614 & & & & & 9.0 & & \\
\hline 45 & 22.61211 & 78.49324 & $\begin{array}{l}0.028 \\
0.275\end{array}$ & 0.2047056 & 0.57466214 & 2706852 & 68.3 & 76.0 & $\begin{array}{l}0.99596 \\
0.62962\end{array}$ \\
\hline 46 & 23.13545 & 80.24029 & & & & & 4.3 & & \\
\hline
\end{tabular}

MAD, data obtained using :

$$
J_{n}=\frac{1+\gamma G}{2 \pi} \Sigma_{\text {Qpoles }}\left\{\begin{array}{c}
\cos \left(\gamma G \alpha_{i}\right) \\
\sin \left(\gamma G \alpha_{i}\right)
\end{array}\right\}(K L)_{i} z_{c o, i}
$$




\section{I built a "Xing factory" from all that material, applicable to AGS and RHIC :}

- Starting from a MAD/TWISS file,

a sheet is produced, which containing strengths, vertical closed orbit etc. :

\begin{tabular}{|c|c|c|c|c|c|c|}
\hline & $+/-Q z$ & $\mathrm{Jn}^{\wedge}$ & ^2 $\mathrm{Nn}^{\wedge} 2 / \mathrm{ep}$ & $z \max$ & vkick & $2 \mathrm{pi}-2 \mathrm{pi}$ \\
\hline 0 & +8.7648 & 0.0 & 127.3933 & $0.0000 \mathrm{E}+00$ & $0.0000 \mathrm{E}+00$ & $0.2032 E-10$ \\
\hline 12 & +8.7648 & 0.0 & 39.2391 & $0.0000 \mathrm{E}+00$ & $0.0000 \mathrm{E}+00$ & $0.2032 E-10$ \\
\hline 4 & -8.7648 & 0.0 & 0.3019 & $0.0000 \mathrm{E}+00$ & $0.0000 \mathrm{E}+00$ & $0.2032 \mathrm{E}-10$ \\
\hline 24 & +8.7648 & 0.0 & 0.9011 & $0.0000 \mathrm{E}+00$ & $0.0000 \mathrm{E}+00$ & $0.2032 \mathrm{E}-10$ \\
\hline 36 & -8.7648 & 0.0 & 321.0361 & $0.0000 \mathrm{E}+00$ & $0.0000 \mathrm{E}+00$ & $0.2032 E-10$ \\
\hline 36 & +8.7648 & 0.0 & 1937.6307 & $0.0000 \mathrm{E}+00$ & $0.0000 \mathrm{E}+00$ & $0.2032 \mathrm{E}-10$ \\
\hline & -8.7648 & 0.0 & 4.87452 & $0.0000 \mathrm{E}+00$ & $0.0000 \mathrm{E}+00$ & $0.2032 \mathrm{E}-10$ \\
\hline
\end{tabular}

Strengths from lattice functions are computed using the regular thin-lens modelling :

- closed orbit : $\left(J_{n} / \hat{z}\right)=\frac{1+\gamma G}{2 \pi} \Sigma_{\text {Qpoles }}\left\{\begin{array}{c}\cos \left(\gamma G \alpha_{i}\right) \\ \sin \left(\gamma G \alpha_{i}\right)\end{array}\right\}(K L)_{i} z_{c o, i} / \hat{z}$

- intrinsic : $\left(J_{n}^{ \pm} / \sqrt{\left.\epsilon_{z} / \pi\right)}=\frac{1+\gamma G}{4 \pi} \Sigma_{\text {Qpoles }}\left\{\begin{array}{c}\cos \left(\gamma G \alpha_{i} \pm \psi_{i}\right) \\ \sin \left(\gamma G \alpha_{i} \pm \psi_{i}\right)\end{array}\right\}(K L)_{i} \sqrt{\beta_{z, i}}\right.$

Note that the long combined function AGS bends need be split into 4 pieces at least so to get the strengths in that table converged.

- Taking $n \pm \nu_{z}$ from that sheet, and including in addition working conditions for the tracking,

- under the form of a template zgoubi.dat file obtained from MAD translation,

- with specific values for $\hat{V}, \phi_{s}$, etc.

then Zgoubi will perform a scan of all resonances, one after the other.

Initial particle conditions are taken to be :

- closed orbit : $\hat{z}_{c o}=\sqrt{810^{-6}} /\left(J_{n} / \hat{z}_{c o}\right)$

- intrinsic : $\epsilon_{y} / \pi=\sqrt{810^{-6}} /\left(J_{n} / \epsilon_{y}\right)$

with $\sqrt{810^{-6}}$ in there just because $2 \exp \left(-\frac{\pi}{2} \frac{\left|J_{n}\right|^{2}}{\alpha}\right)-1 \approx 0.5$ (given $\alpha \approx 4.4110^{-5}$ ) is a convenient value for appropriate accuracy when drawing $p_{f} / p_{i}$ from the tracking data.

The distance $\Delta$ upstream of the resonance where to start from is given by (in units of $J_{n}$ ) $\Delta /\left|J_{n}\right|=S_{z} / \sqrt{1-S_{z}^{2}}$

taking for instance $S_{z}=1-10^{-4}$, which translates into a number of turns using $\gamma G=\Delta+\left(n \pm \nu_{z}\right)$ and the acceleration rate, $d E$ per turn.

This tool produces the typical following table :

\begin{tabular}{|c|c|c|c|c|c|c|c|c|}
\hline & $\&$ & Energy & $\&$ & $\mathrm{Qz}$ & $\&$ & e_z/pi & $\&$ & p_init \\
\hline & $\&$ & $(\mathrm{GeV})$ & $\&$ & & $\&$ & $(1 e-6)$ & $\&$ & \\
\hline $0+Q z$ & $\&$ & 4.2359 & $\&$ & 0.76345 & $\&$ & $3.604 \mathrm{E}-02$ & $\&$ & 1.000 \\
\hline $12+Q z$ & $\&$ & 10.516 & $\&$ & 0.76345 & $\&$ & 0.123 & $\&$ & 1.000 \\
\hline $24-Q z$ & $\&$ & 7.6222 & $\&$ & 0.76490 & $\varepsilon$ & 15.8 & $\&$ & 0.9994 \\
\hline $24+Q z$ & $\&$ & 16.796 & $\&$ & 0.76331 & $\&$ & 5.42 & $\&$ & 0.9972 \\
\hline $36-Q z$ & $\&$ & 13.902 & $\&$ & 0.76346 & $\&$ & $1.516 \mathrm{E}-02$ & $\&$ & 1.000 \\
\hline $36+Q z$ & $\&$ & 23.076 & $\&$ & 0.76346 & $\&$ & $2.537 \mathrm{E}-03$ & $\&$ & 1.000 \\
\hline $48-Q z$ & $\&$ & 20.182 & $\&$ & 0.76343 & $\&$ & 1.01 & $\&$ & 0.9992 \\
\hline
\end{tabular}

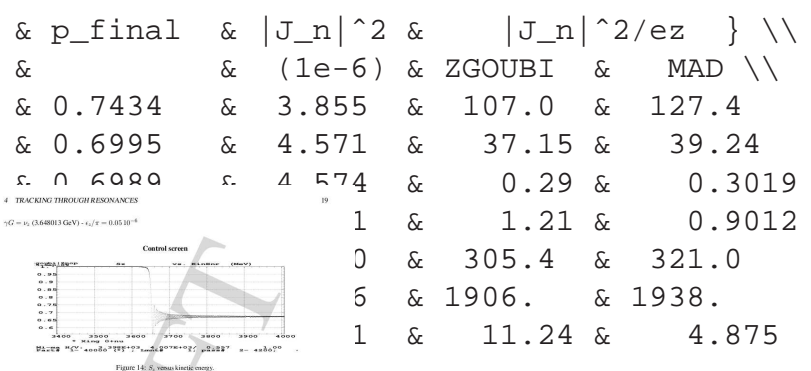

which includes provision for comparison between MAD and output data.

Tunes and emittances are "measured" in the vicinity of the crossing gamma, namely, considering a few hundred turns around $\left(n \pm \nu_{z}\right) / G$.

This tool also produces a "control panel" :

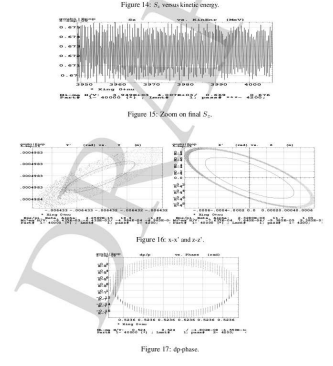




\section{Tracking through resonances}

\subsection{Intrinsic resonances}

\subsection{1 $\gamma G=\nu_{z}(3.648013 \mathrm{GeV})$}

\section{Tracking data}

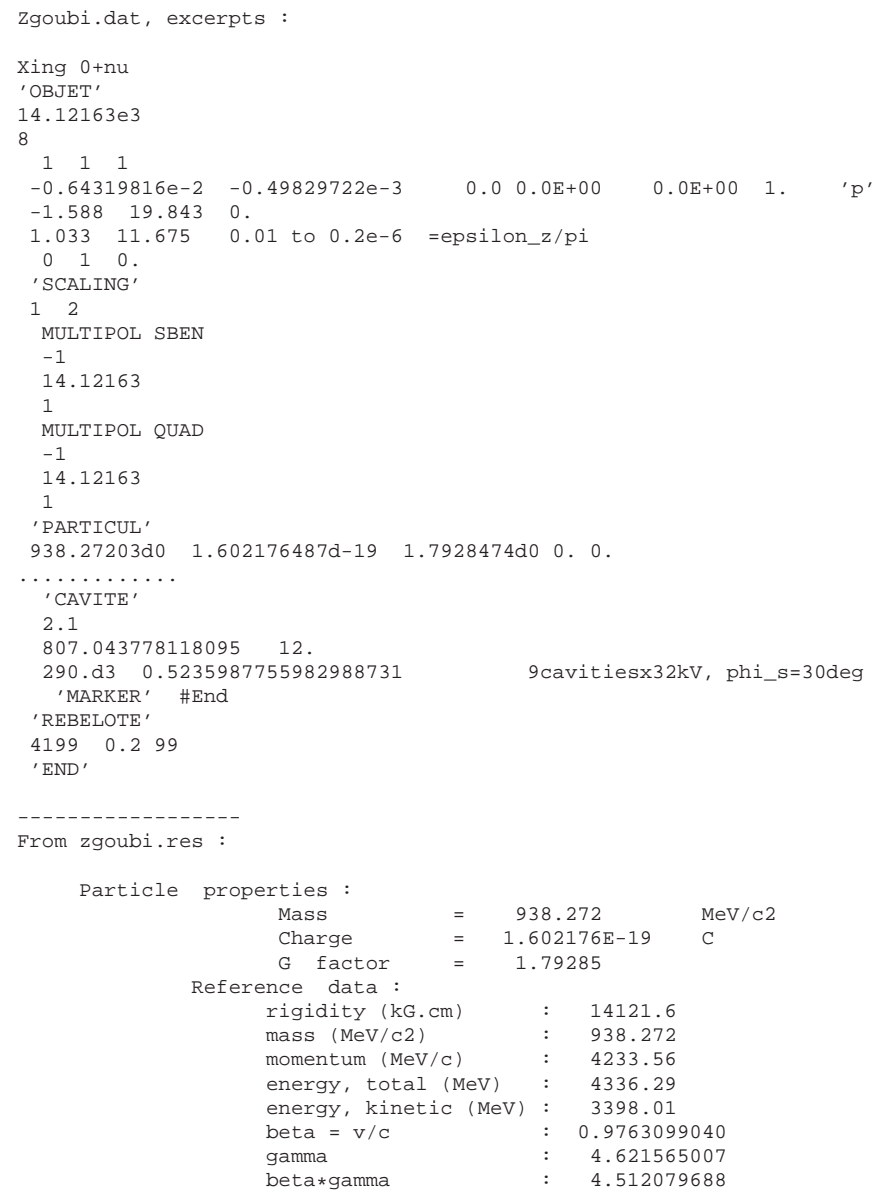

\section{Strengths}

From Figs. 10-23 one gets

$\begin{array}{ccccccc}\begin{array}{c}\epsilon_{z} / \pi \\ \left(10^{-6}\right)\end{array} & A^{2} & \begin{array}{c}\left|J_{n}\right|^{2} \\ \left(10^{-5}\right)\end{array} & A^{2} / \epsilon_{z} / \pi & \left|J_{n}\right|^{2} / \epsilon_{z} / \pi & p_{\text {init }} & p_{\text {final }} \\ 0.01 & 0.0357308 & 1.0030567 & 3573080 & 100.3057 & 1 & 0.9298 \\ 0.05 & 0.1788277 & 5.0201593 & 3576553 & 100.4032 & 1 & 0.6725 \\ 0.2 & 0.7467644 & 2.0963627 & 3733822 . & 104.8181 & 0.9999 & -5.22 \mathrm{E}-02\end{array}$


$\gamma G=\nu_{z}(3.648013 \mathrm{GeV})-\epsilon_{z} / \pi=0.0110^{-6}$

\section{Control screen}

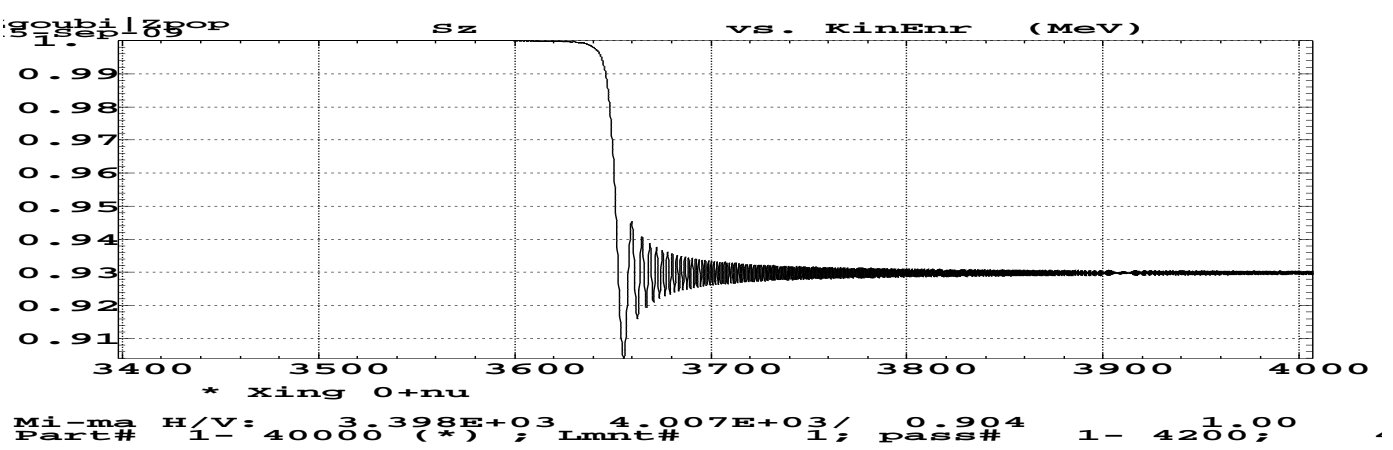

Figure 10: $S_{z}$ versus kinetic energy.

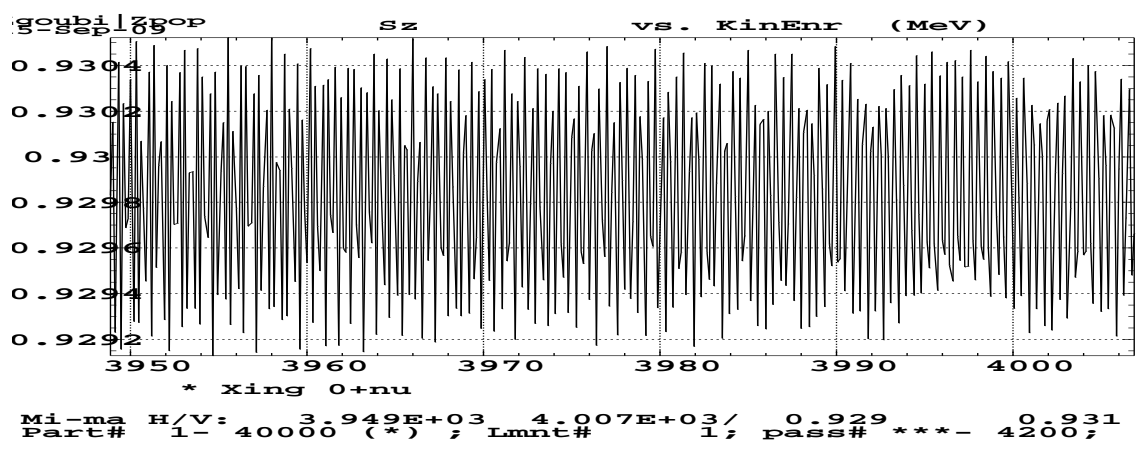

Figure 11: Zoom on final $S_{z}$.
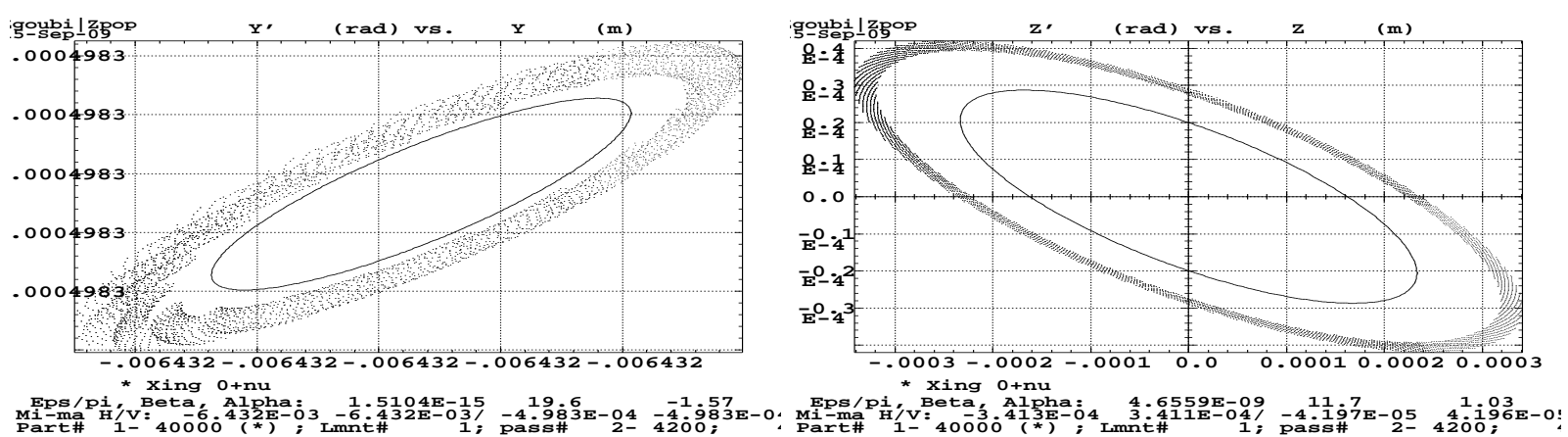

Figure 12: $x-x^{\prime}$ and $z-z^{\prime}$.

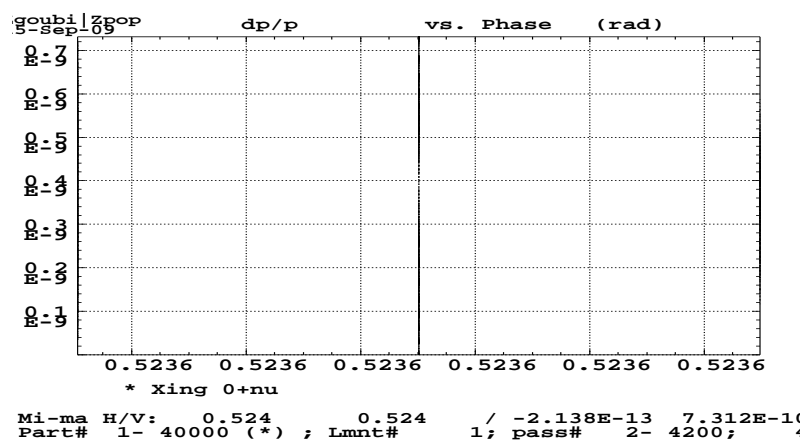

Figure 13: dp-phase. 
$\gamma G=\nu_{z}(3.648013 \mathrm{GeV})-\epsilon_{z} / \pi=0.0510^{-6}$

\section{Control screen}

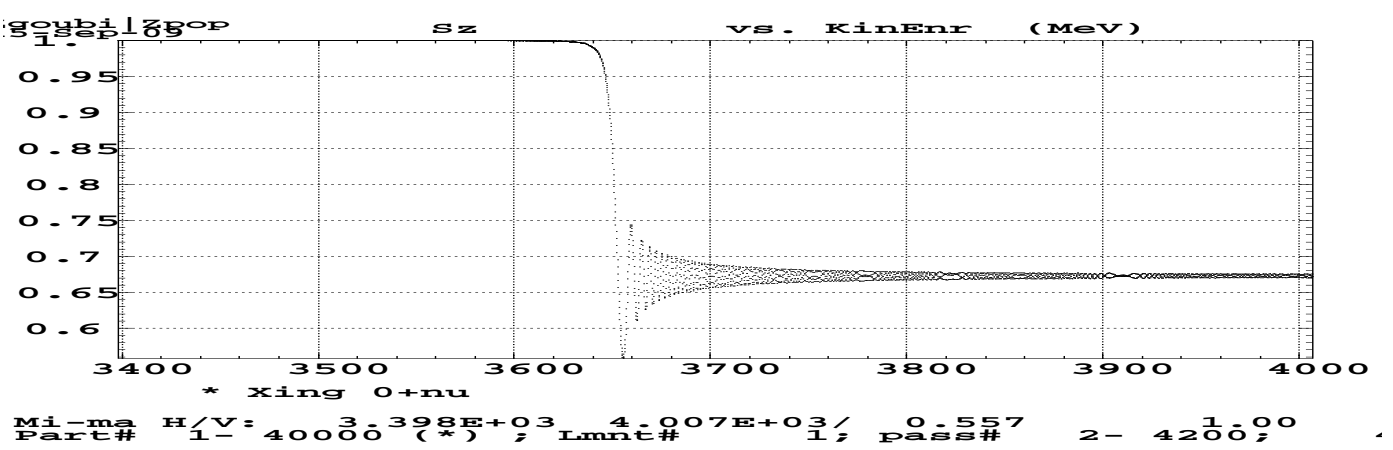

Figure 14: $S_{z}$ versus kinetic energy.

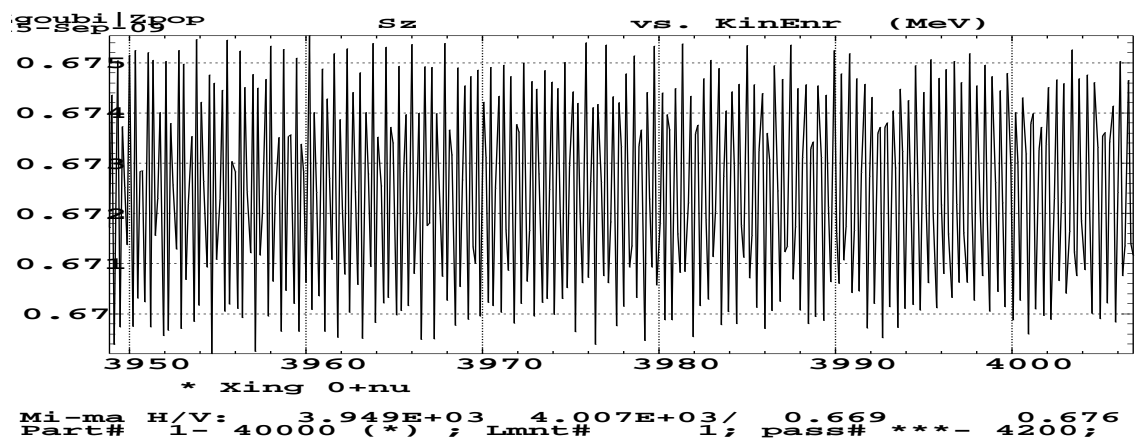

Figure 15: Zoom on final $S_{z}$.
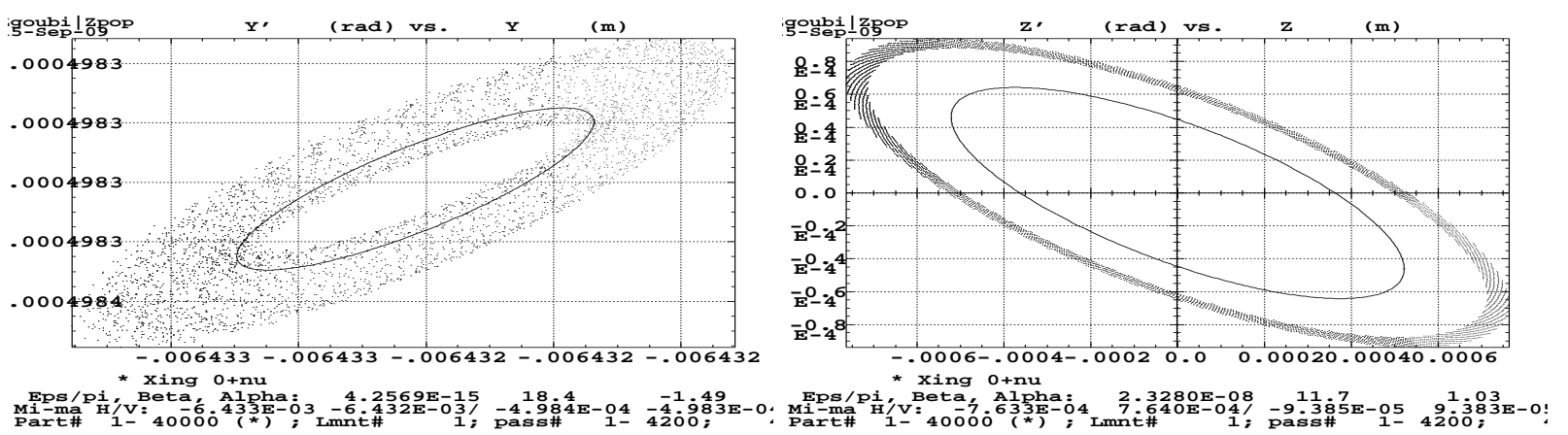

Figure 16: $\mathrm{x}-\mathrm{x}^{\prime}$ and $\mathrm{z}-\mathrm{z}^{\prime}$.

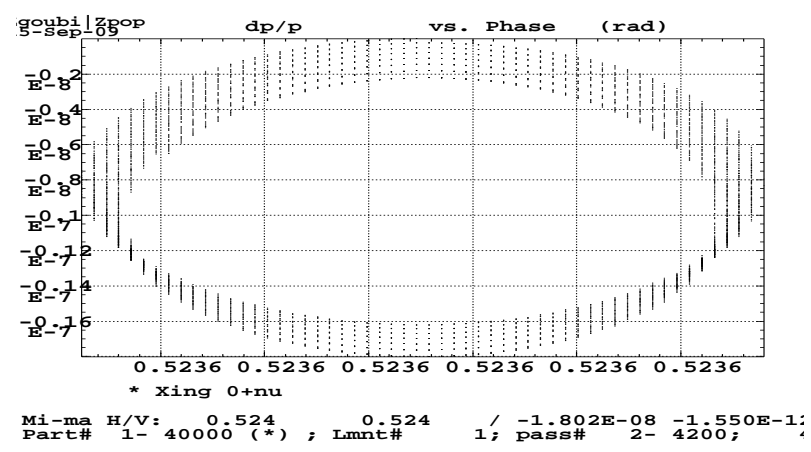

Figure 17: dp-phase. 
$\gamma G=\nu_{z}(3.648013 \mathrm{GeV})-\epsilon_{z} / \pi=0.210^{-6}$

\section{Control screen}

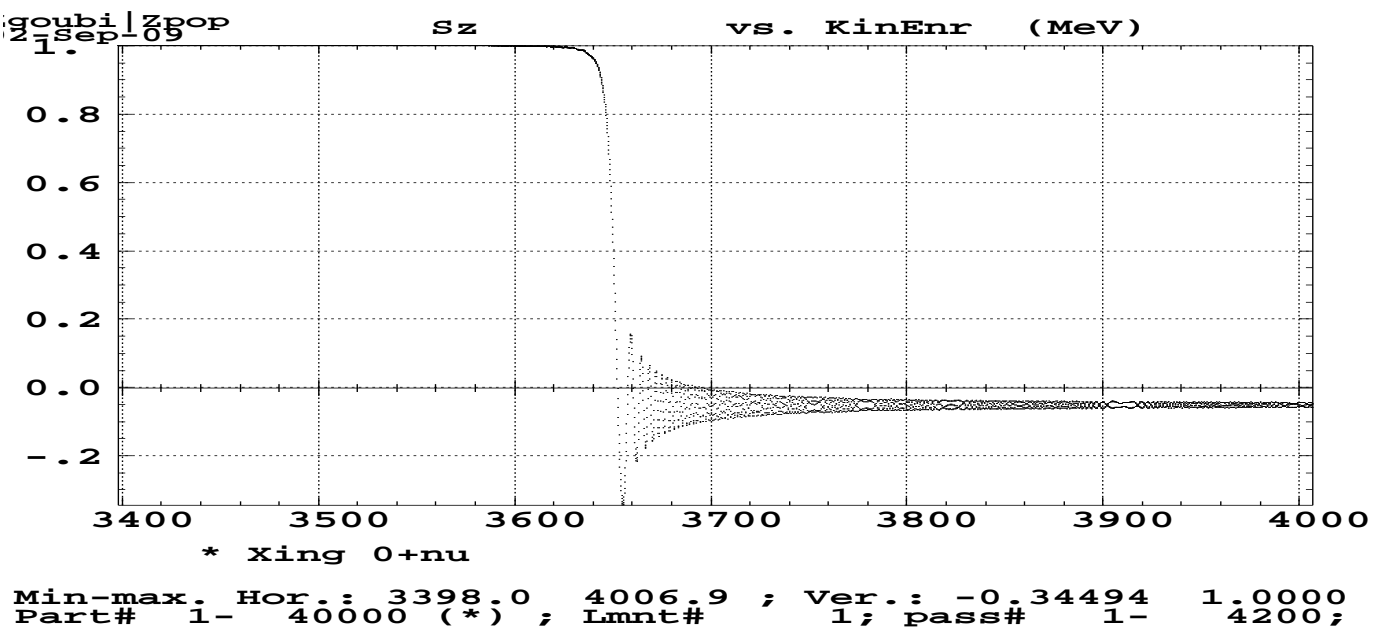

Figure 18: $S_{z}$ versus kinetic energy.

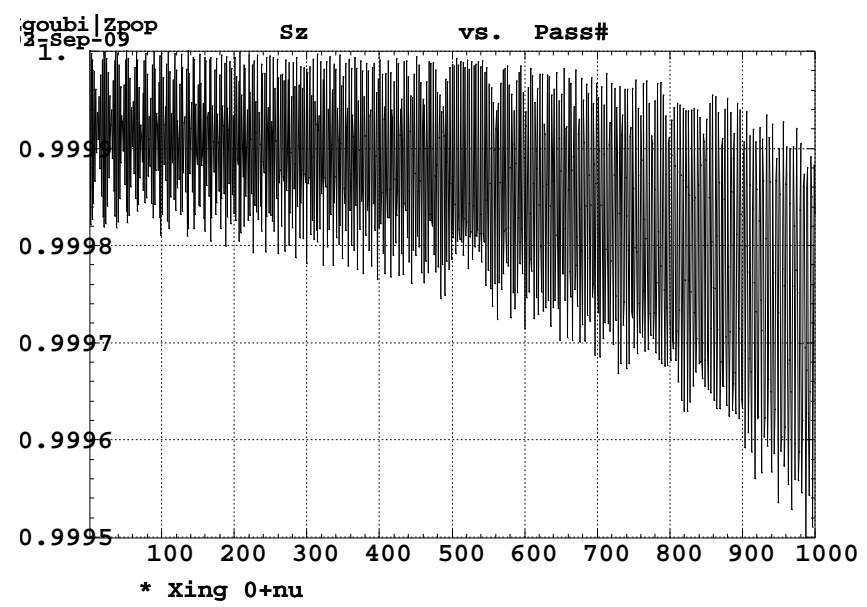

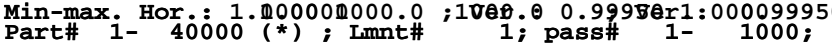

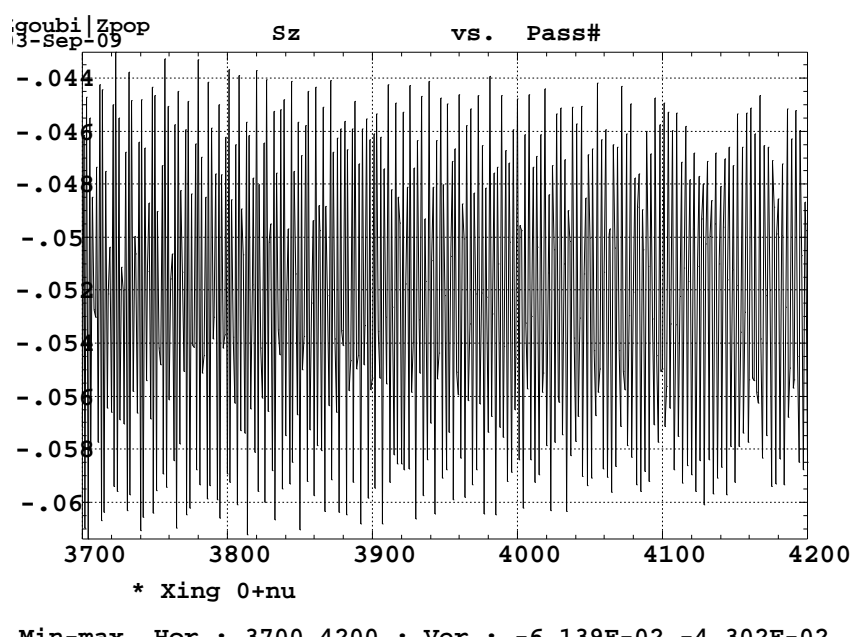

$\underset{\operatorname{Min}-\max }{\operatorname{Hor} \#}{ }_{40}{ }_{40000} 37004200 ;$ Ver.: $-6.139 \mathrm{E}-02-4.302 \mathrm{E}-02$

Figure 19: Zoom on initial $S_{z}$.

Figure 20: Zoom on final $S_{z}$.

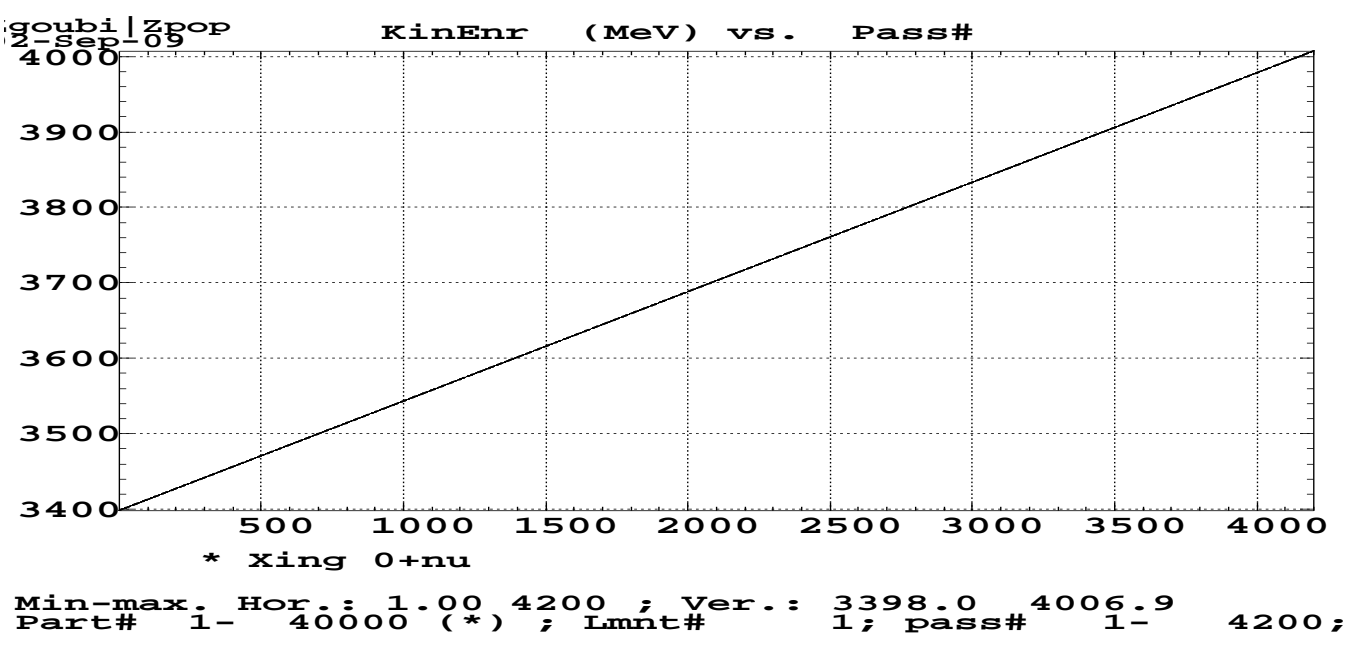

Figure 21: Kinetic E versus turn number. 
$\gamma G=\nu_{z}(3.648013 \mathrm{GeV})-\epsilon_{z} / \pi=0.210^{-6}$

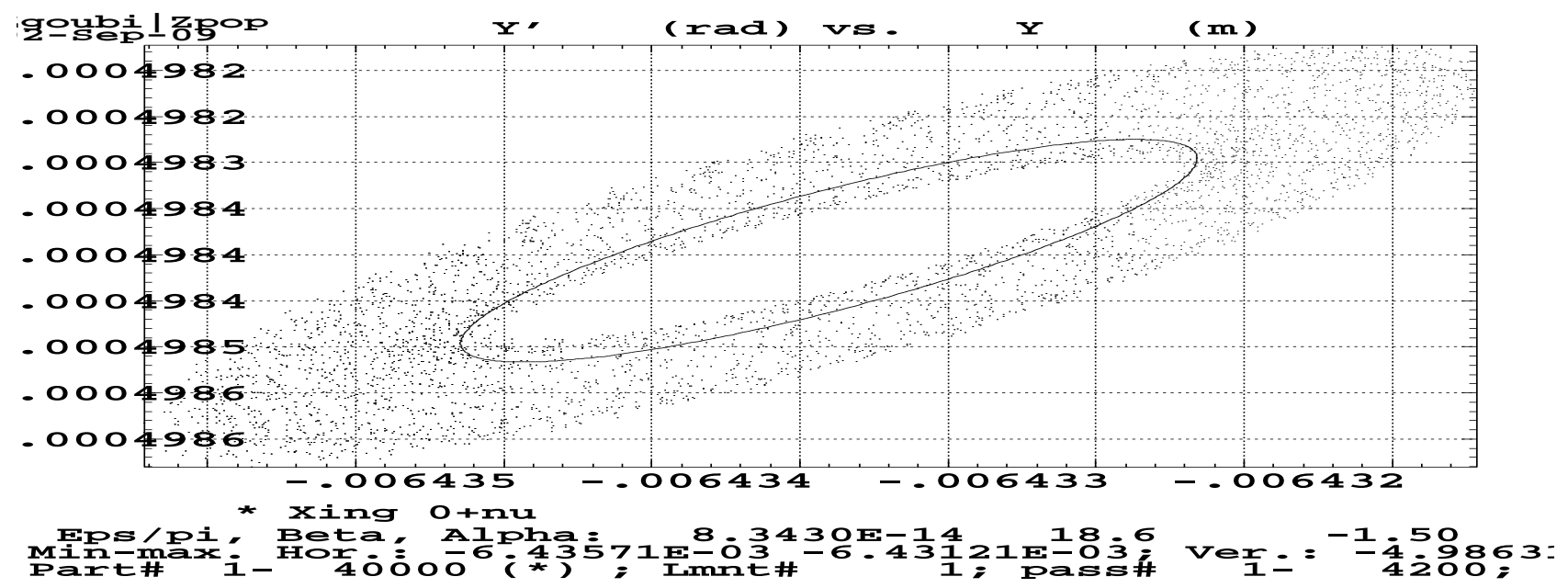

Figure 22: $x-x^{\prime}$.

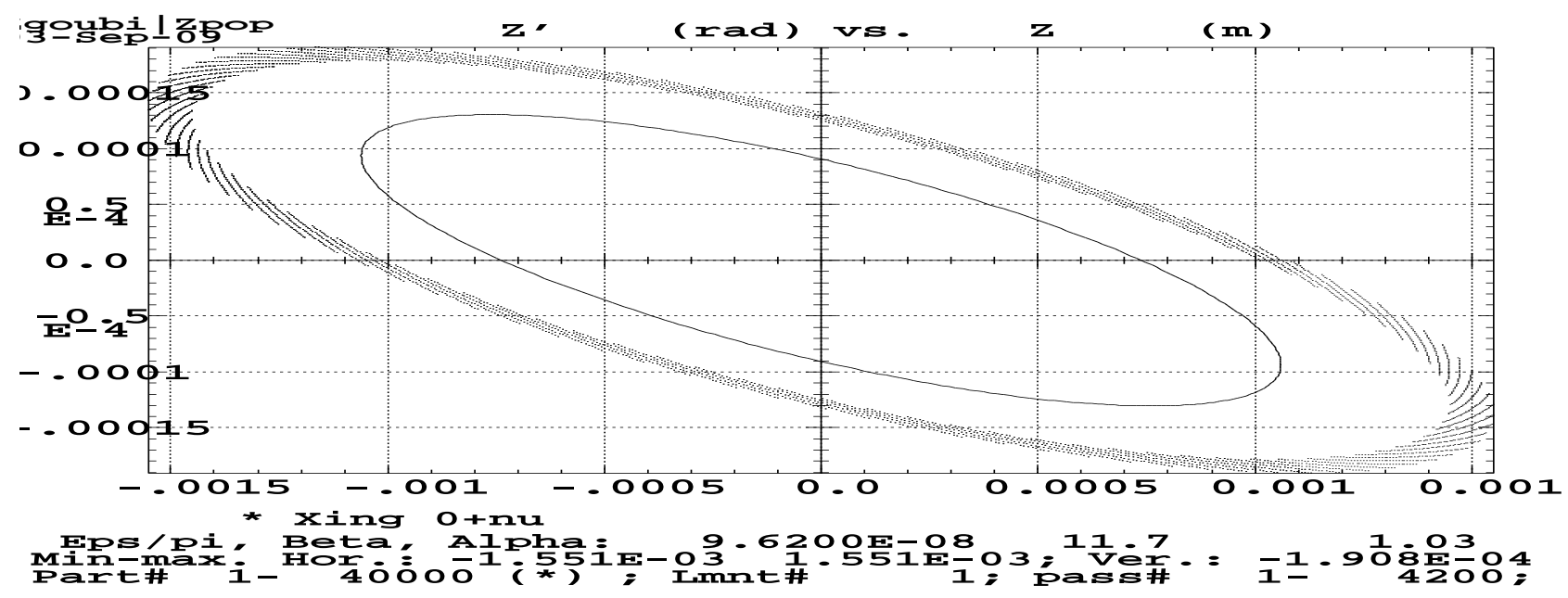

Figure 23: z-z'.

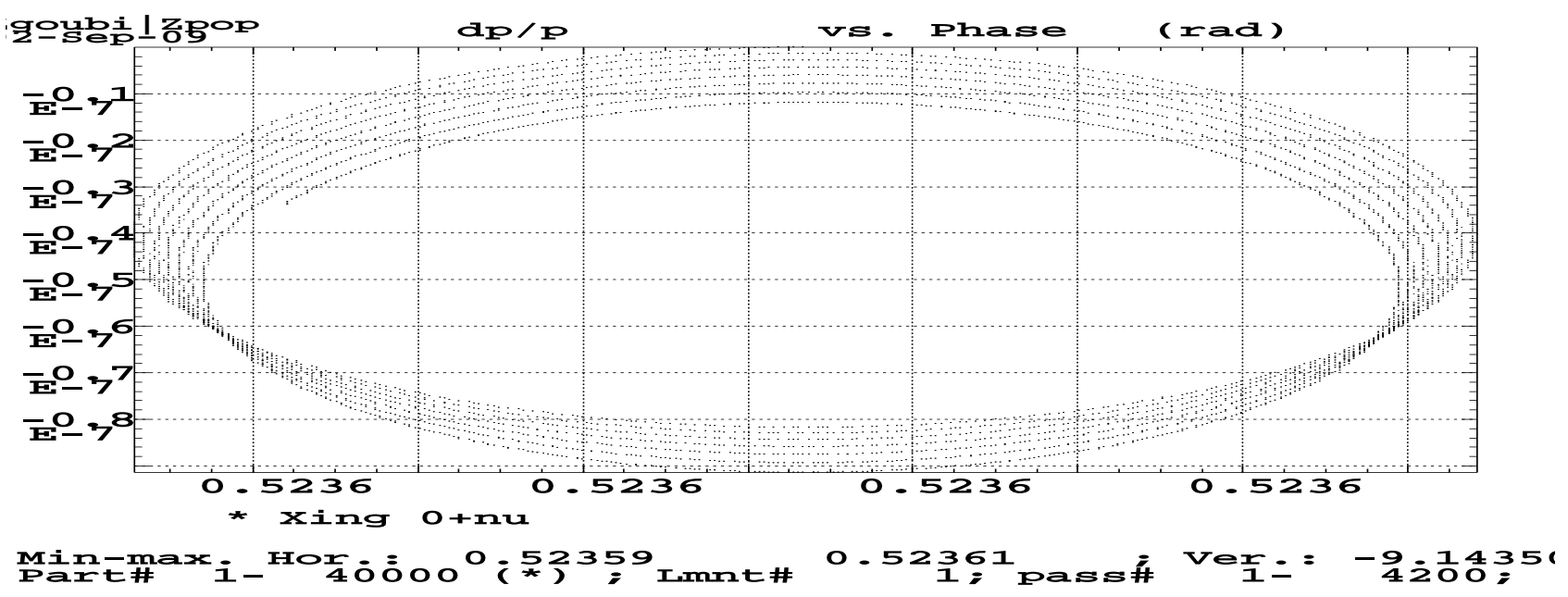

Figure 24: dp-phase. 


\section{Testing possible effect of betatron phase}

7 particles evenly spread on $\epsilon_{z} / \pi=0.2 \mathrm{~mm} . \mathrm{mrad}$ invariant are launched : no observable difference in spin dynamics.

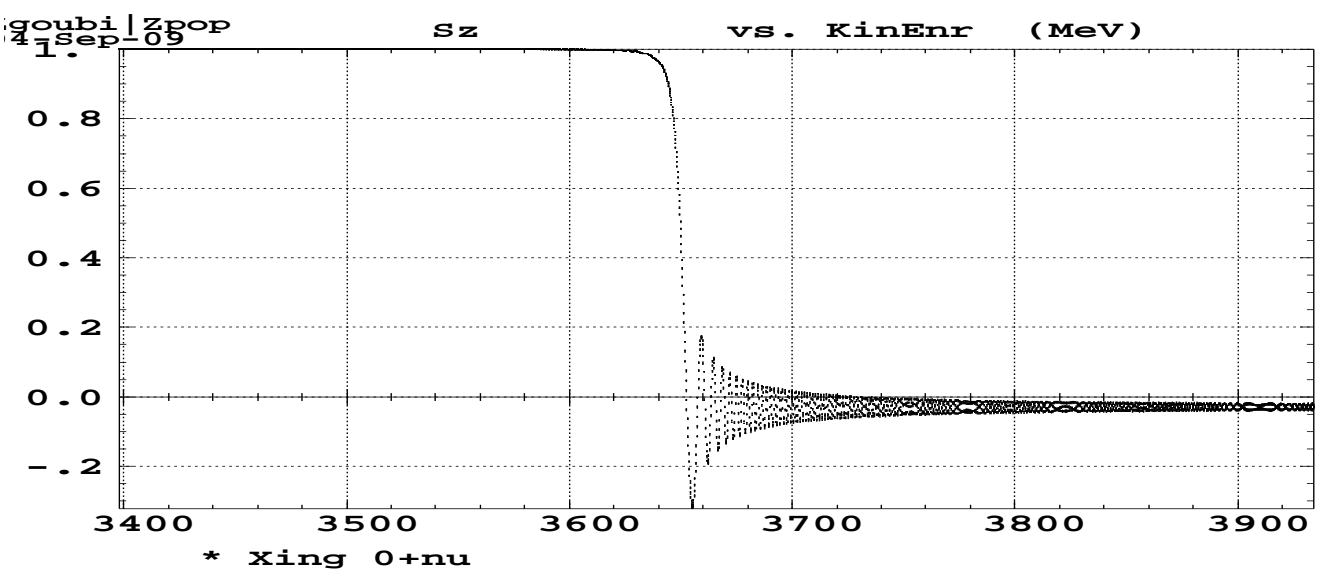

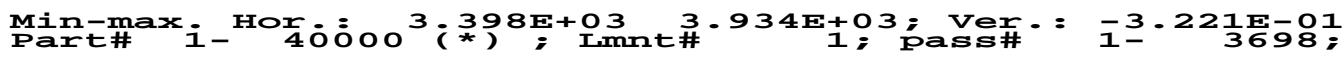

Figure 25: $S_{z}$ versus kinetic energy, 7 particles evenly spread on $\epsilon_{z} / \pi=0.2 \mathrm{~mm}$.mrad invariant. 


\subsection{2 $\gamma G=24-\nu_{z}(\mathbf{7 . 8 2 8 9 2} \mathbf{G e V})$}

\section{Tracking data}

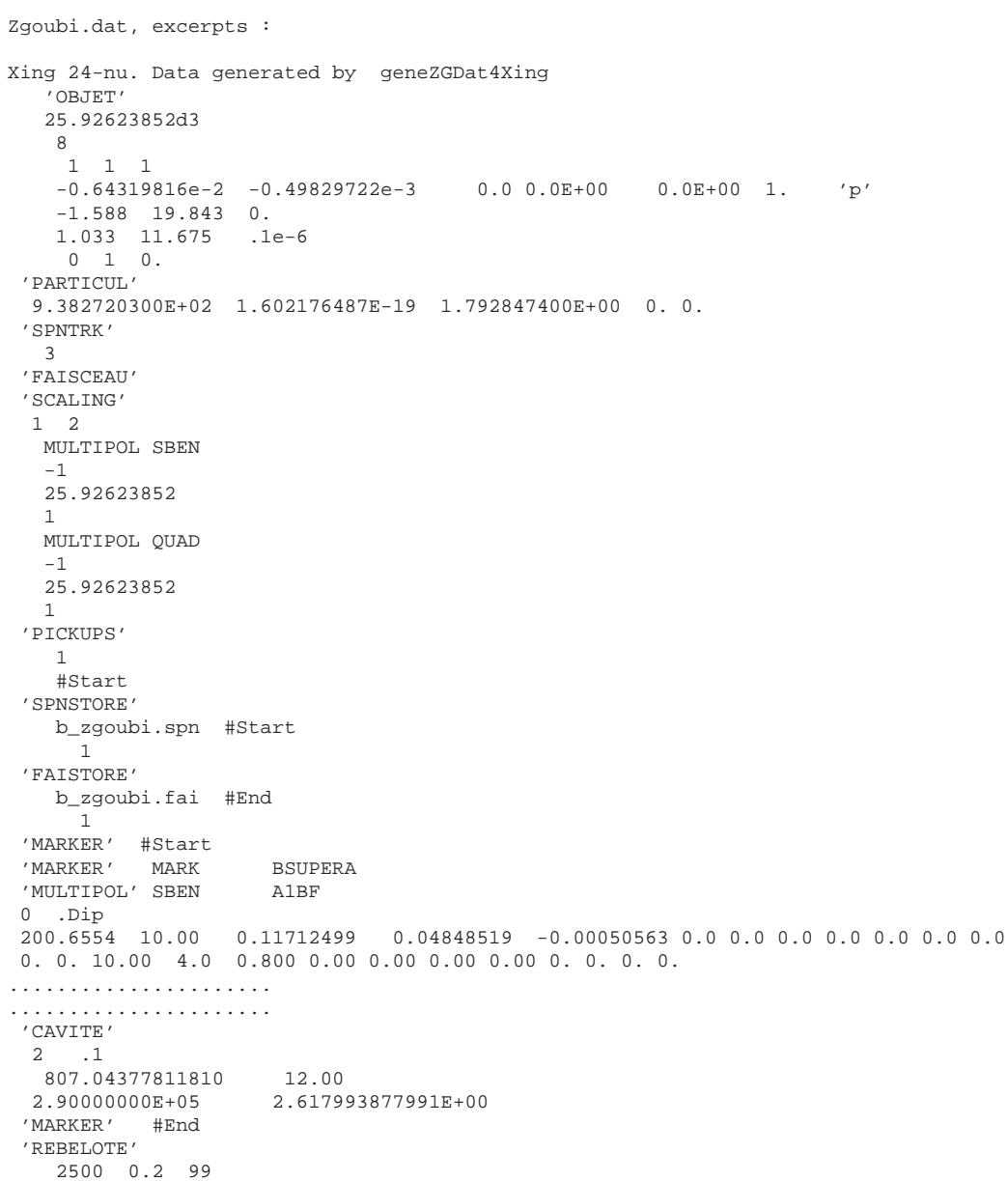

\section{Strengths}
$\epsilon_{z} / \pi$
$A^{2}$
$\left|J_{n}\right|^{2}$
$A^{2} / \epsilon_{z} / \pi$
$\left|J_{n}\right|^{2} / \epsilon_{z} / \pi$
$p_{\text {init }}$
$p_{\text {final }}$
$\left(10^{-6}\right)$ $\left(10^{-6}\right)$
$\begin{array}{lllllll}0.1 & 1.050547 \mathrm{E}-03 & 2.94915967 \mathrm{E}-2 & 10505 & 0.29491597 & 1 . & 0.997900\end{array}$ 
$\gamma G=24-\nu_{z}(7.82892 \mathrm{GeV})-\epsilon_{z} / \pi=0.110^{-6}$

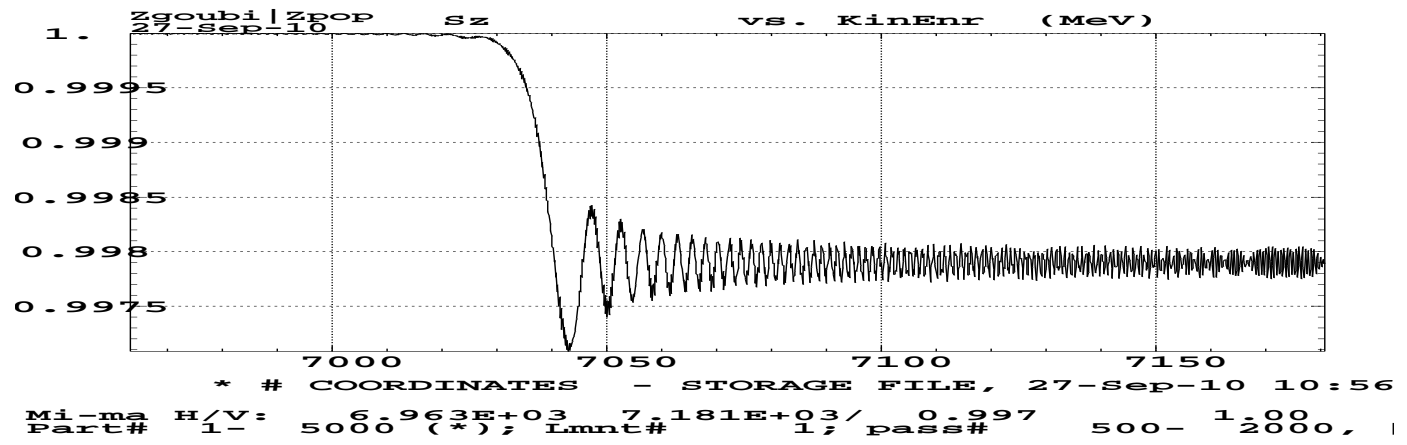

Figure 26: $S_{z}$ versus kinetic energy.
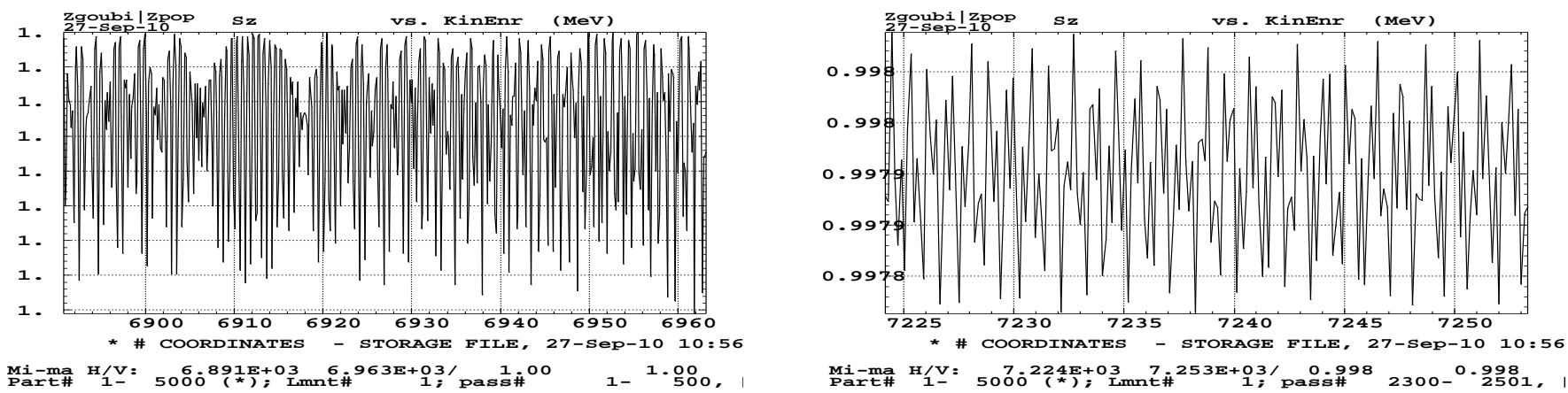

Figure 27: Zoom on initial $S_{z}$.

Figure 28: Zoom on final $S_{z}$.

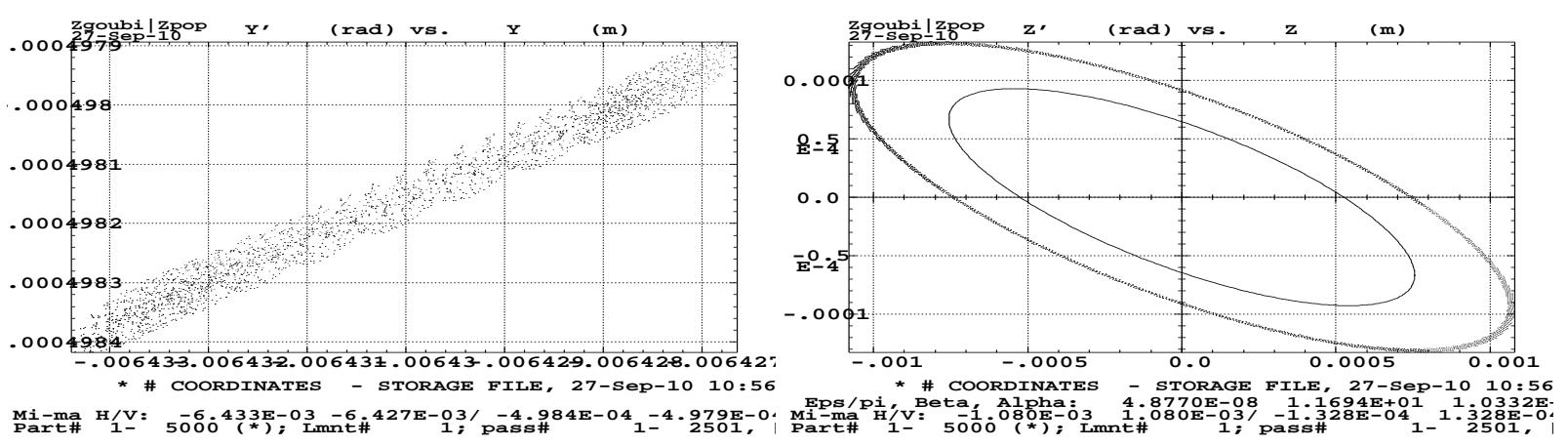

Figure 29: $x-x^{\prime}$ and $z-z^{\prime}$.

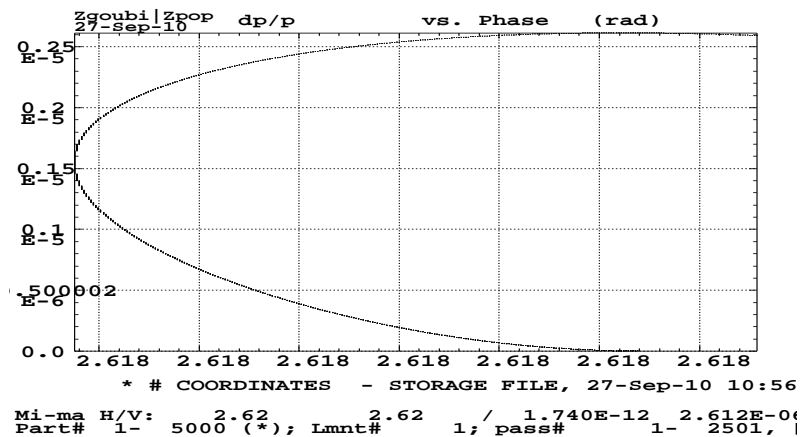

Figure 30: dp-phase. 


\subsection{3 $\gamma G=12+\nu_{z}(\mathbf{7 . 8 2 8 9 2} \mathbf{~ G e V})$}

\section{Tracking data}

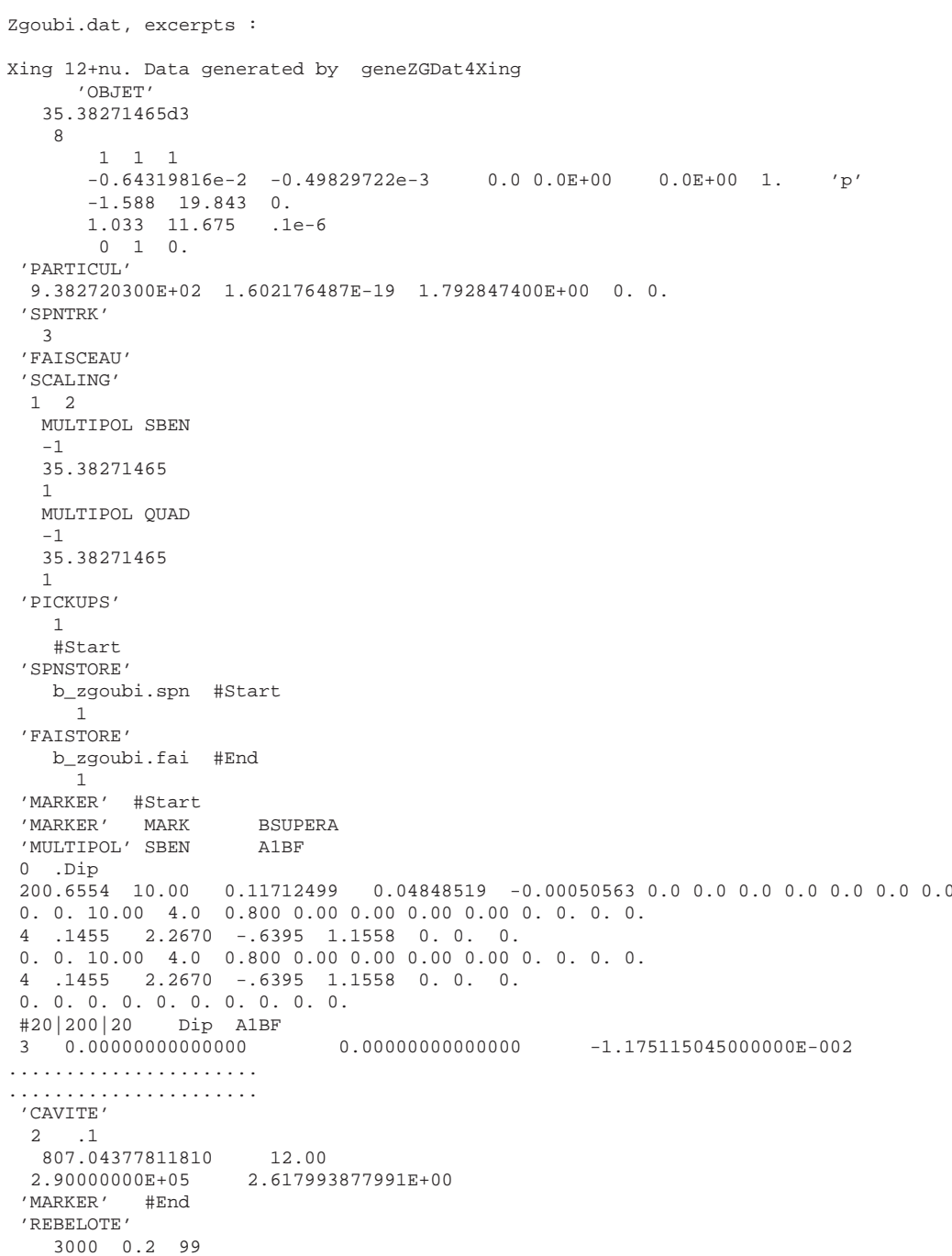

\section{Strengths}

$\begin{array}{ccccccc}\epsilon_{z} / \pi & A^{2} & \left|J_{n}\right|^{2} & A^{2} / \epsilon_{z} / \pi & \left|J_{n}\right|^{2} / \epsilon_{z} / \pi & p_{\text {init }} & p_{\text {final }} \\ \left(10^{-6}\right) & & \left(10^{-6}\right) & & & & \\ 0.002 & 0.00256527 & 0.07201394 & 1282637 & 36.006969 & 1 . & 0.994876 \\ 0.1 & 0.12897037 & 3.62053515 & 1289703 & 36.205349 & 1 . & 0.7590 \\ 2 & 2.5431926 & 71.3940608 & 1271596 & 35.697029 & 1 . & -0.842770\end{array}$


$\gamma G=12+\nu_{z}(7.82892 \mathrm{GeV})-\epsilon_{z} / \pi=0.00210^{-6}$

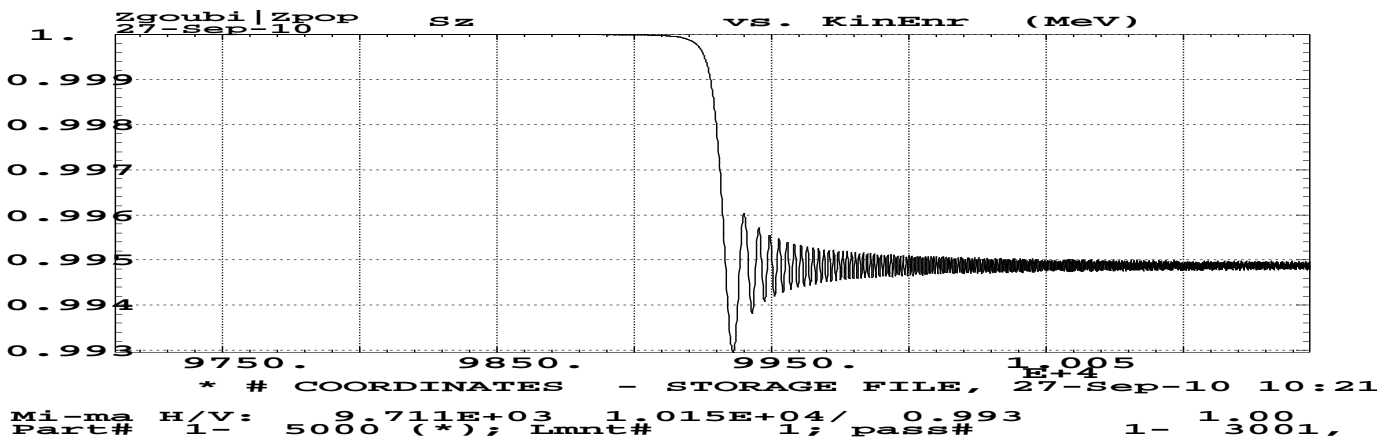

Figure 31: $S_{z}$ versus kinetic energy.

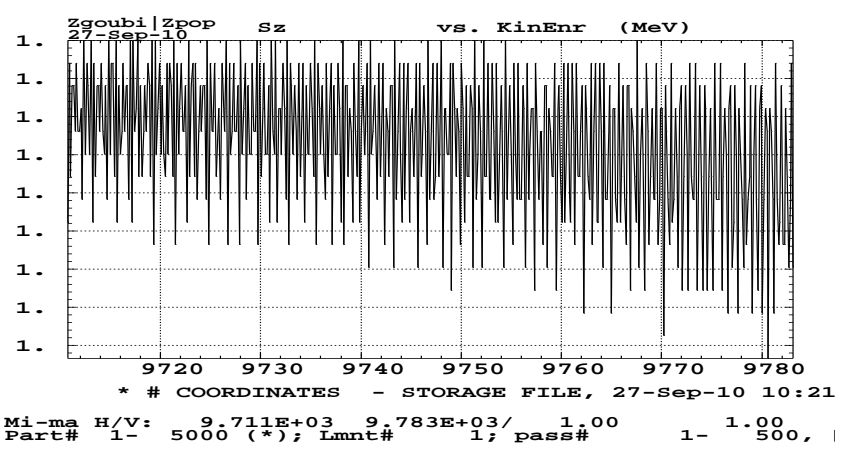

Figure 32: Zoom on initial $S_{z}$.

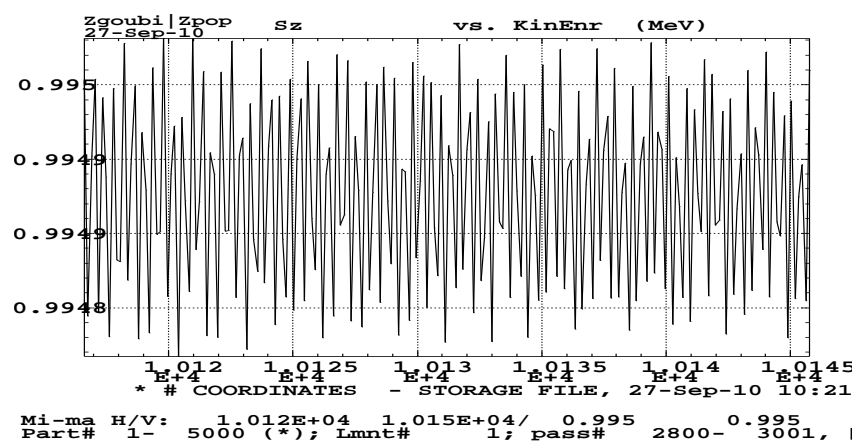

Figure 33: Zoom on final $S_{z}$.

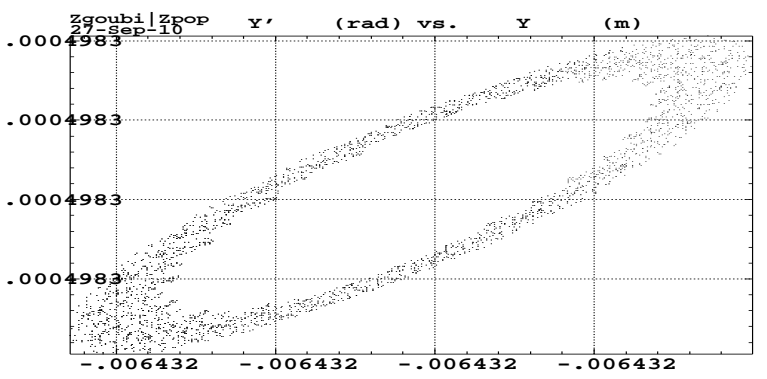

* \# COORDINATES - STORAGE FILE, 27-SeP-10 $10: 21$

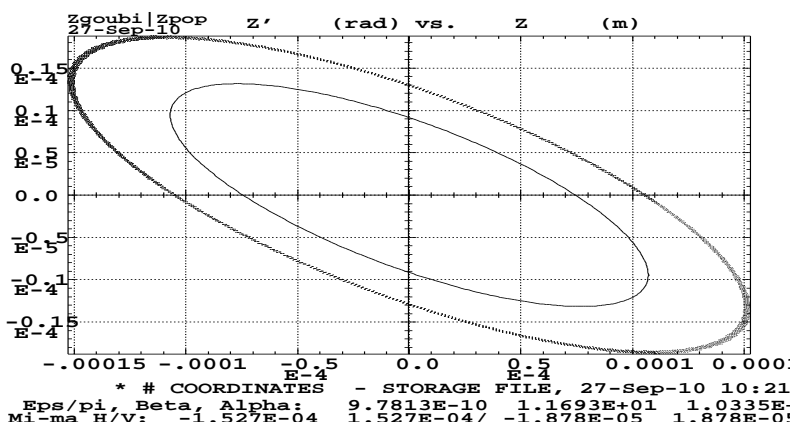

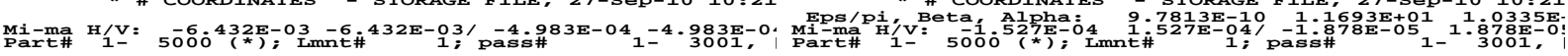

Figure 34: $x-x^{\prime}$ and $z-z^{\prime}$.

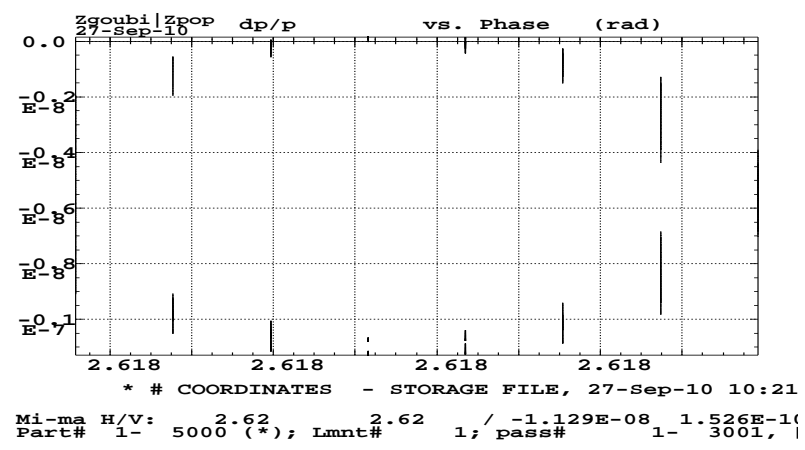

Figure 35: dp-phase. 
$\gamma G=12+\nu_{z}(7.82892 \mathrm{GeV})-\epsilon_{z} / \pi=210^{-6}$

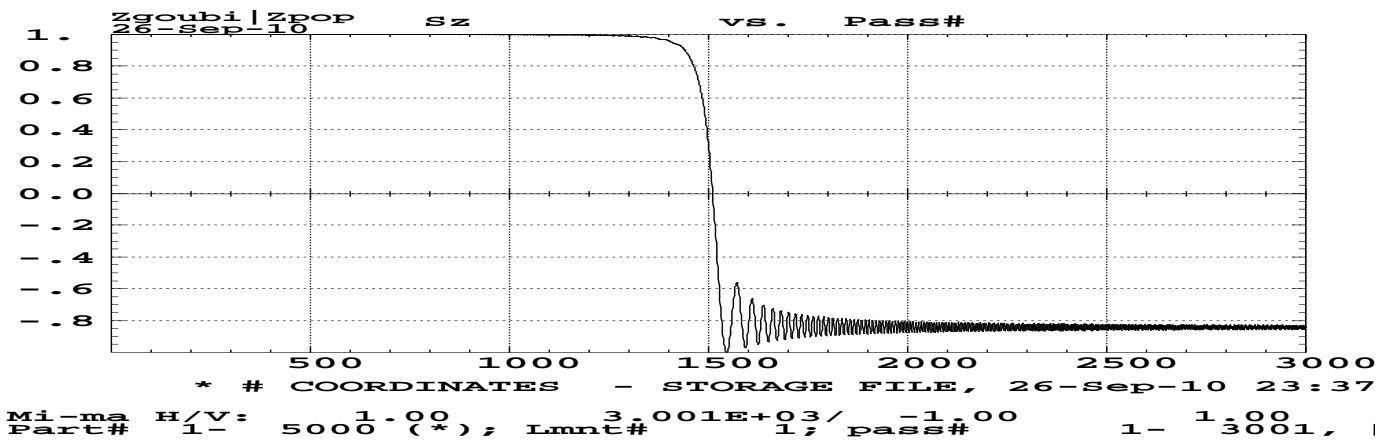

Figure 36: $S_{z}$ versus kinetic energy.

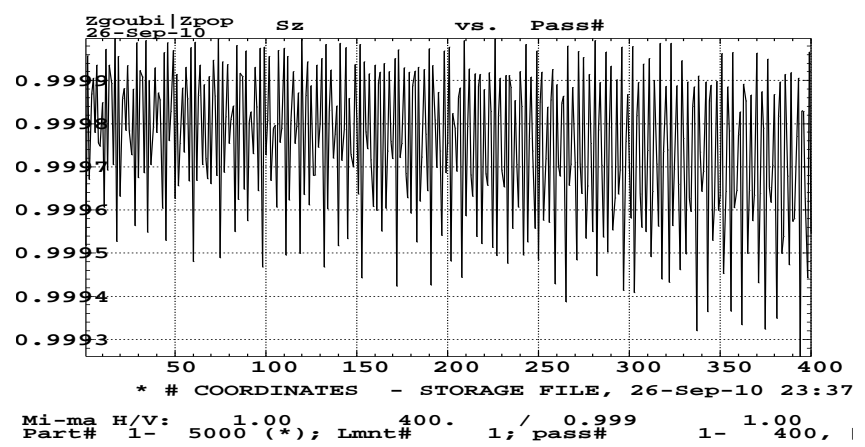

Figure 37: Zoom on initial $S_{z}$.

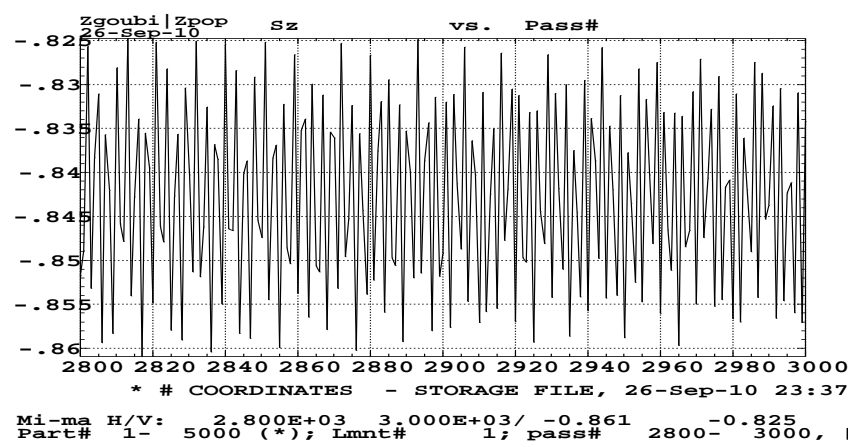

Figure 38: Zoom on final $S_{z}$.

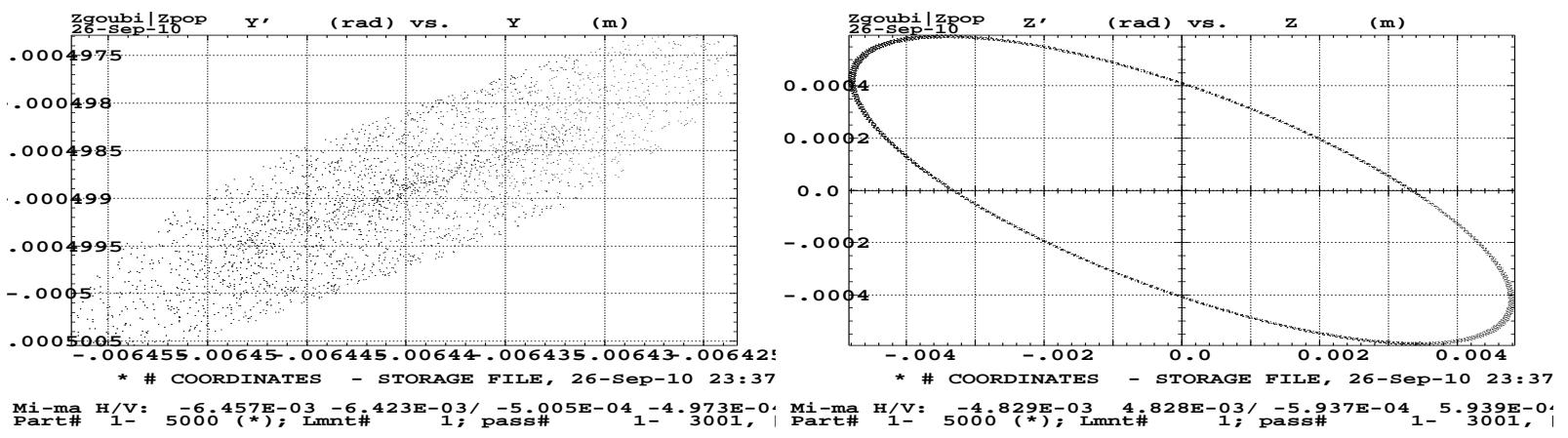

Figure 39: $x-x^{\prime}$ and $z-z^{\prime}$.

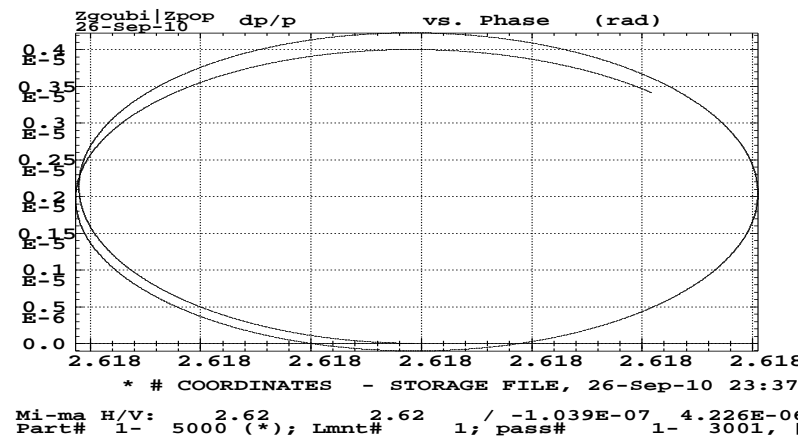

Figure 40: dp-phase. 


\subsection{4 $\gamma G=23+\nu_{z}(\mathbf{1 5 . 6 8 4 8 7} \mathbf{G e V})$}

\section{Tracking data}

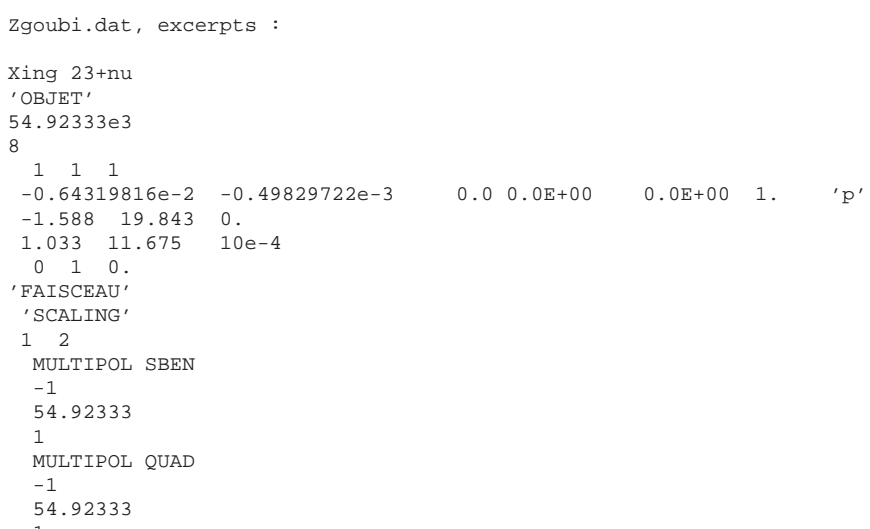

\section{Strength}

No visible resonance, $S_{z} \simeq 1, \forall G \gamma$, up to unreasonable $\mathrm{V}$ emittances... 


\subsection{5 $\gamma G=24+\nu_{z}(\mathbf{1 6 . 2 0 8 2 2} \mathbf{~ G e V})$}

\section{Tracking data}

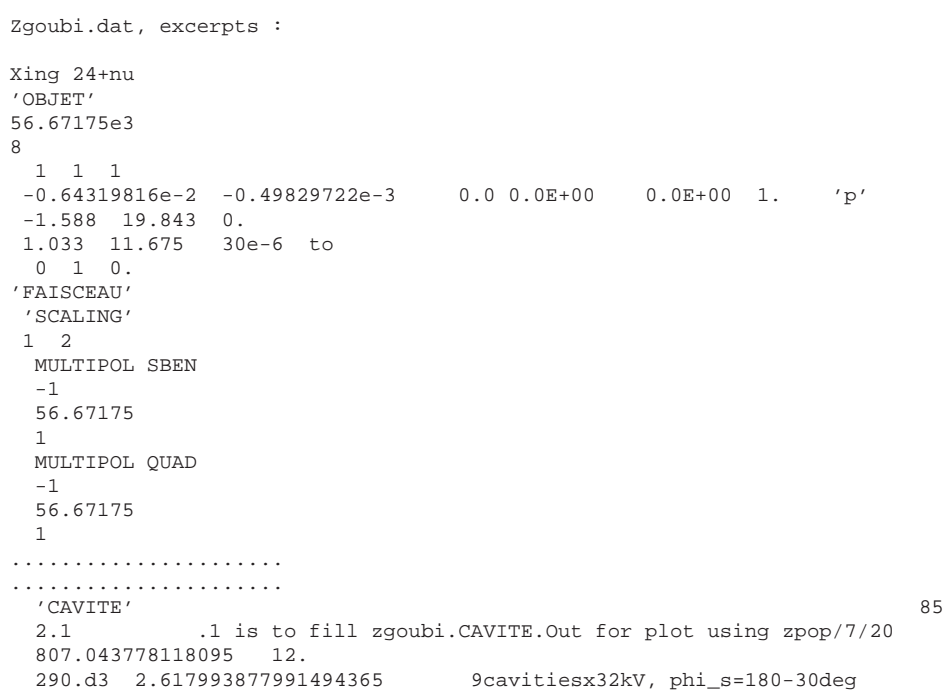

\section{Strengths}

$\begin{array}{ccccccc}\epsilon_{z} / \pi & A^{2} & \left|J_{n}\right|^{2} & A^{2} / \epsilon_{z} / \pi & \left|J_{n}\right|^{2} / \epsilon_{z} / \pi & p_{\text {init }} & p_{\text {final }} \\ \left(10^{-6}\right) & & \left(10^{-5}\right) & & & & \\ 30 & 1.011348 & 2.83912 & 33711.59 & 0.9463723 & 0.987 & -0.2690 \\ 2 & 0.0737230 & 0.20696 & 36861.53 & 1.034799 & 0.999 & 0.857\end{array}$


$\gamma G=24+\nu_{z}(16.20822 \mathrm{GeV})-\epsilon_{z} / \pi=210^{-6}$

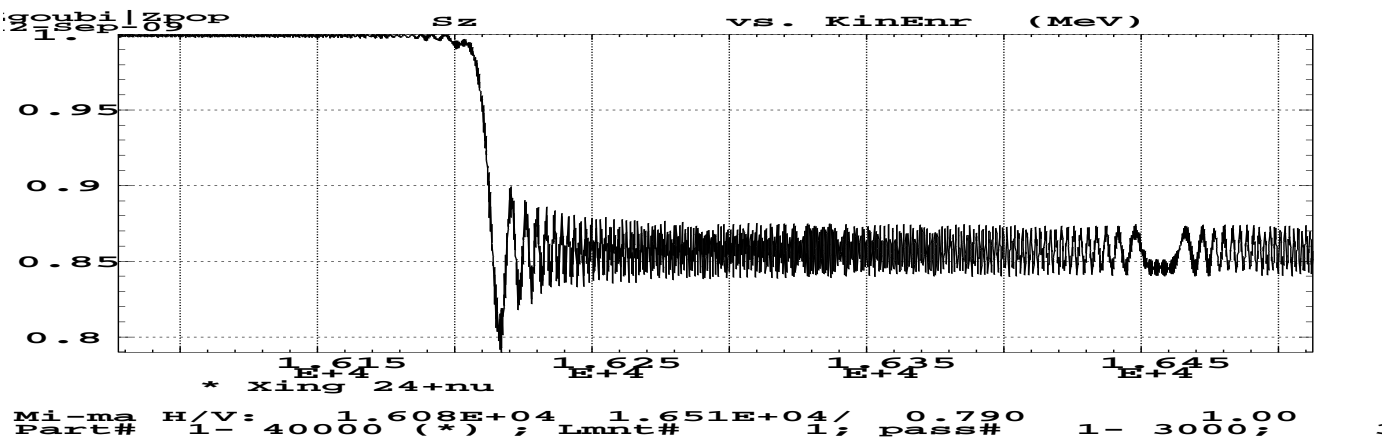

Figure 41: $S_{z}$ versus kinetic energy.

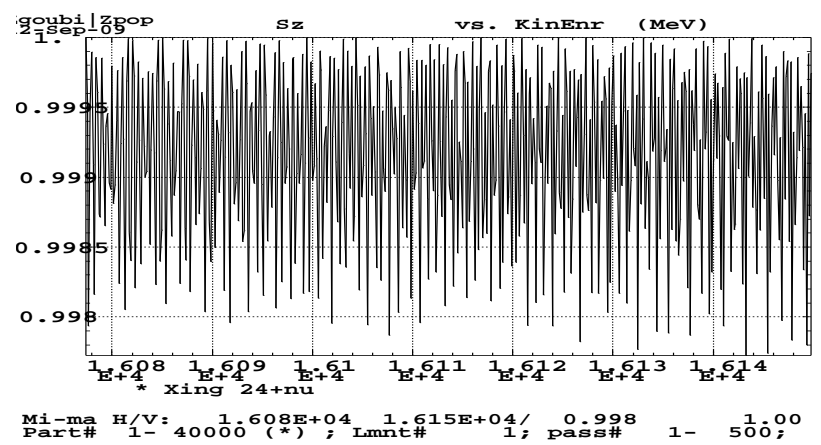

Figure 42: Zoom on initial $S_{z}$.

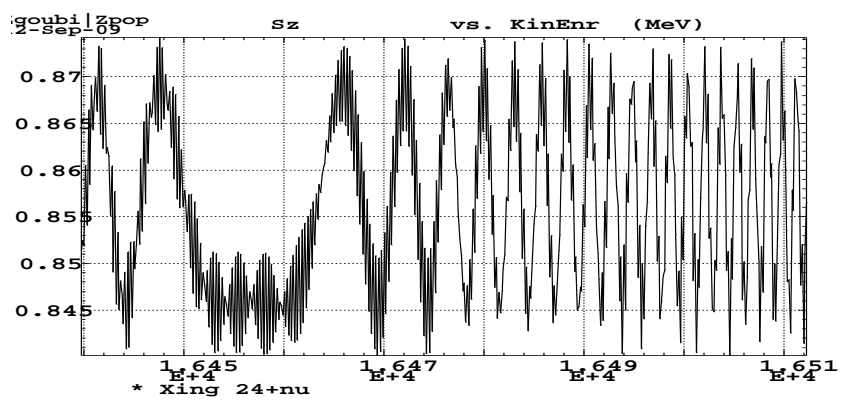

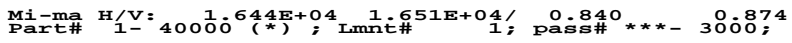

Figure 43: Zoom on final $S_{z}$.

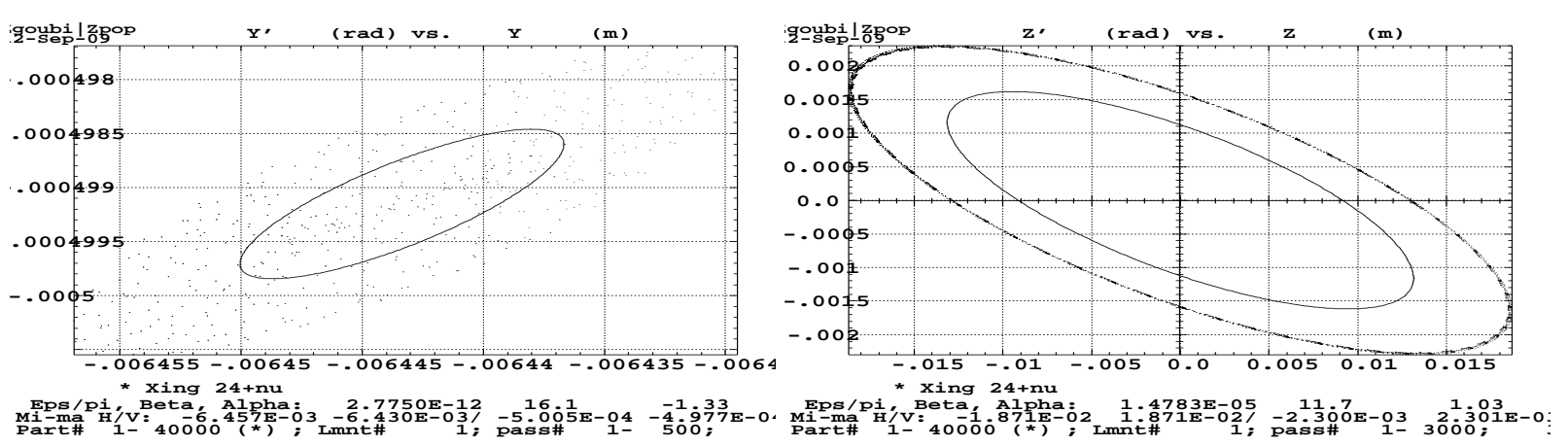

Figure 44: $\mathrm{x}-\mathrm{x}^{\prime}$ and $\mathrm{z}-\mathrm{z}^{\prime}$.

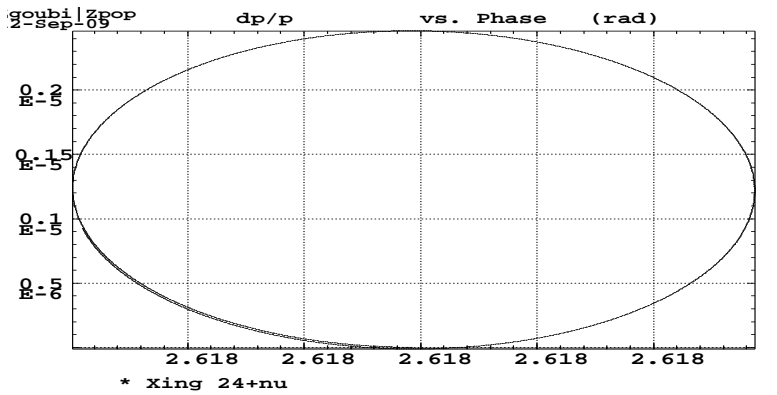

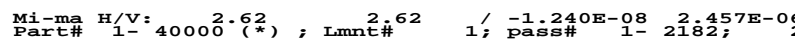

Figure 45: dp-phase. 
$\gamma G=24+\nu_{z}(16.20822 \mathrm{GeV})-\epsilon_{z} / \pi=3010^{-6}$

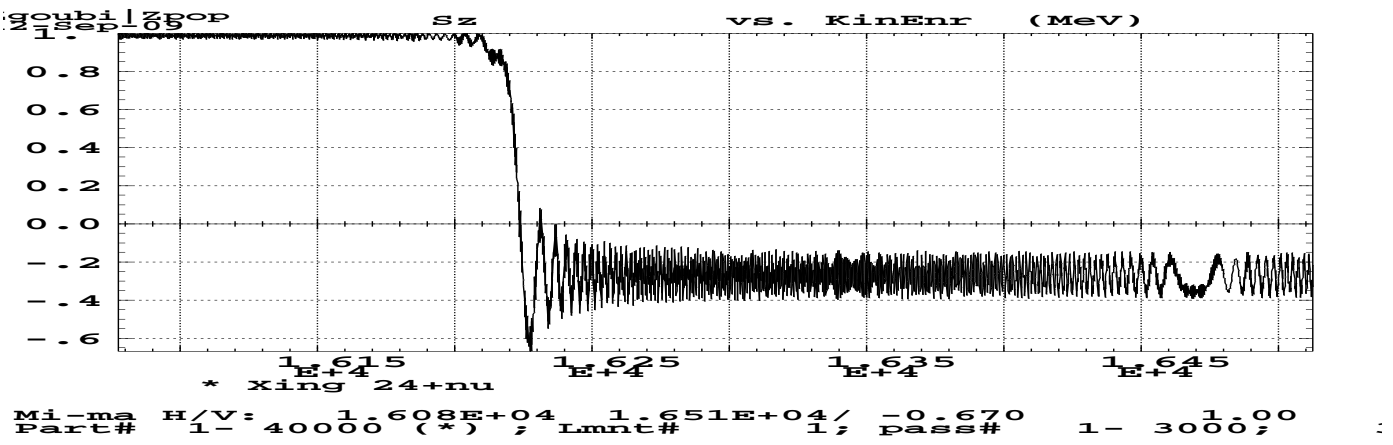

Figure 46: $S_{z}$ versus kinetic energy.

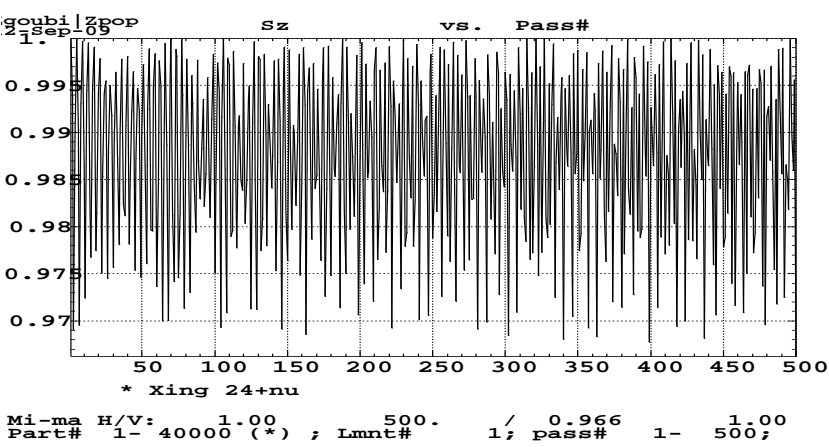

Figure 47: Zoom on initial $S_{z}$.

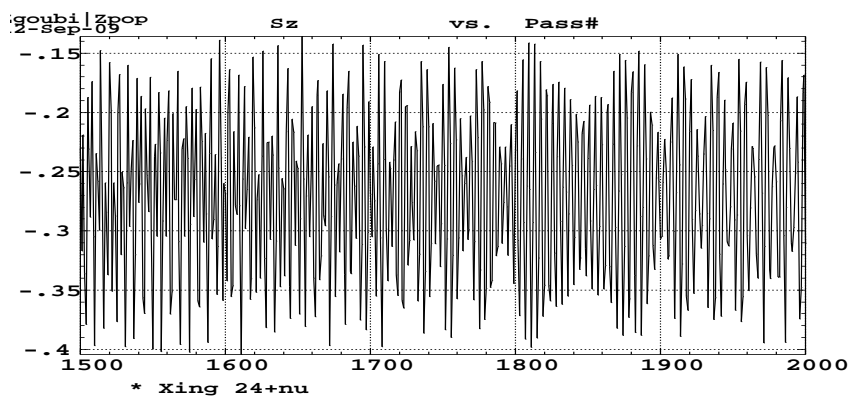

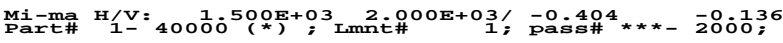

Figure 48: Zoom on final $S_{z}$.
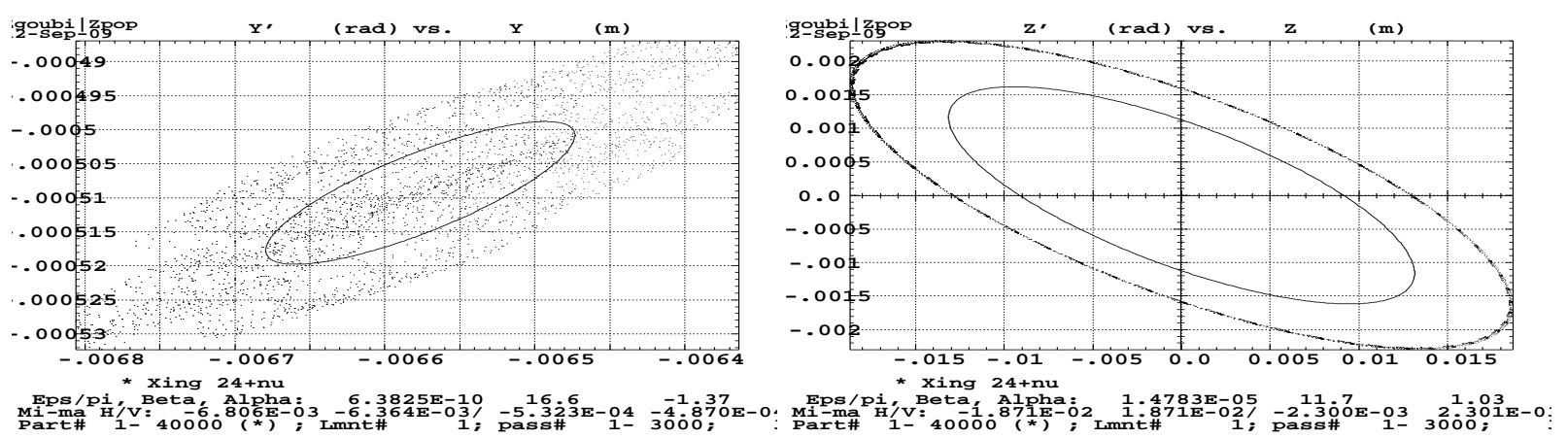

Figure 49: $x-x^{\prime}$ and $z-z^{\prime}$.

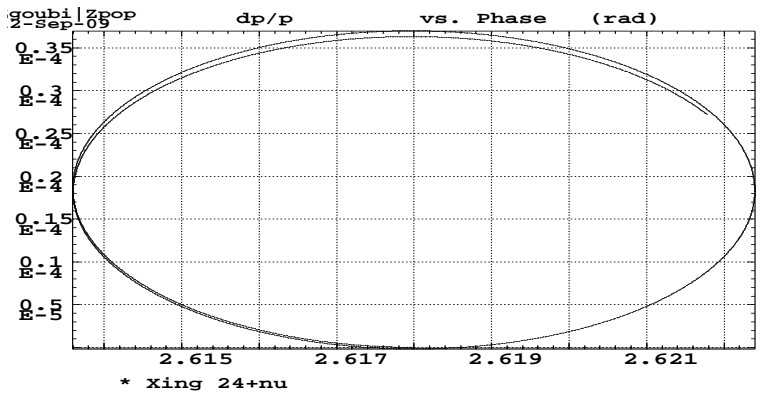

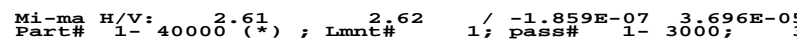

Figure 50: dp-phase. 


\subsection{6 $\gamma G=36-\nu_{z}(\mathbf{1 3 . 3 1 5 7 5} \mathbf{G e V})$}

\section{Tracking data}

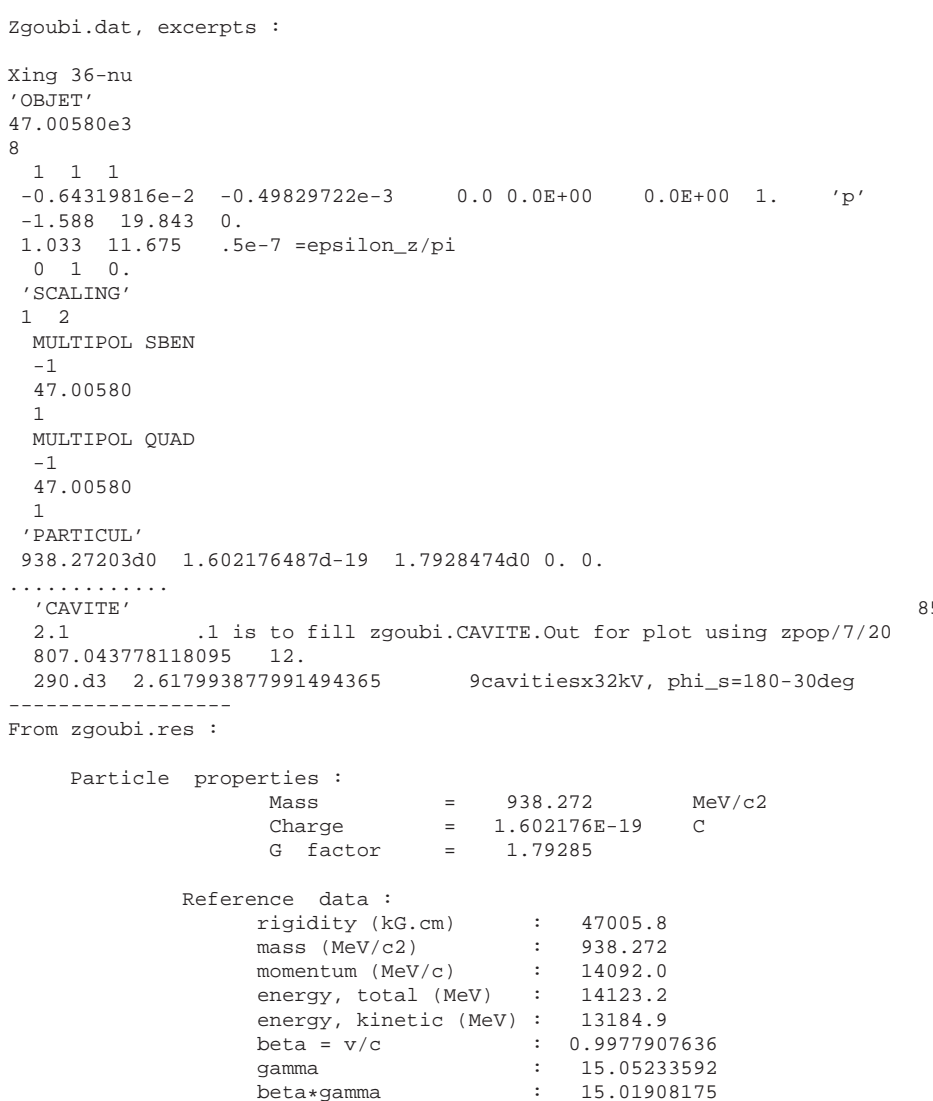

\section{Strength}

From Figs. 52, 53 one gets

$p_{\text {init }} \approx 0.9998300, \quad p_{\text {final }} \approx 0.1545000$

Eq. 2 yields

$$
\begin{aligned}
& A^{2}=0.5494571 \\
& \left|J_{n}\right|^{2}=1.5424697 E-05
\end{aligned}
$$




$$
\gamma G=36-\nu_{z}(\mathbf{1 3 . 3 1 5 7 5} \mathbf{G e V})
$$

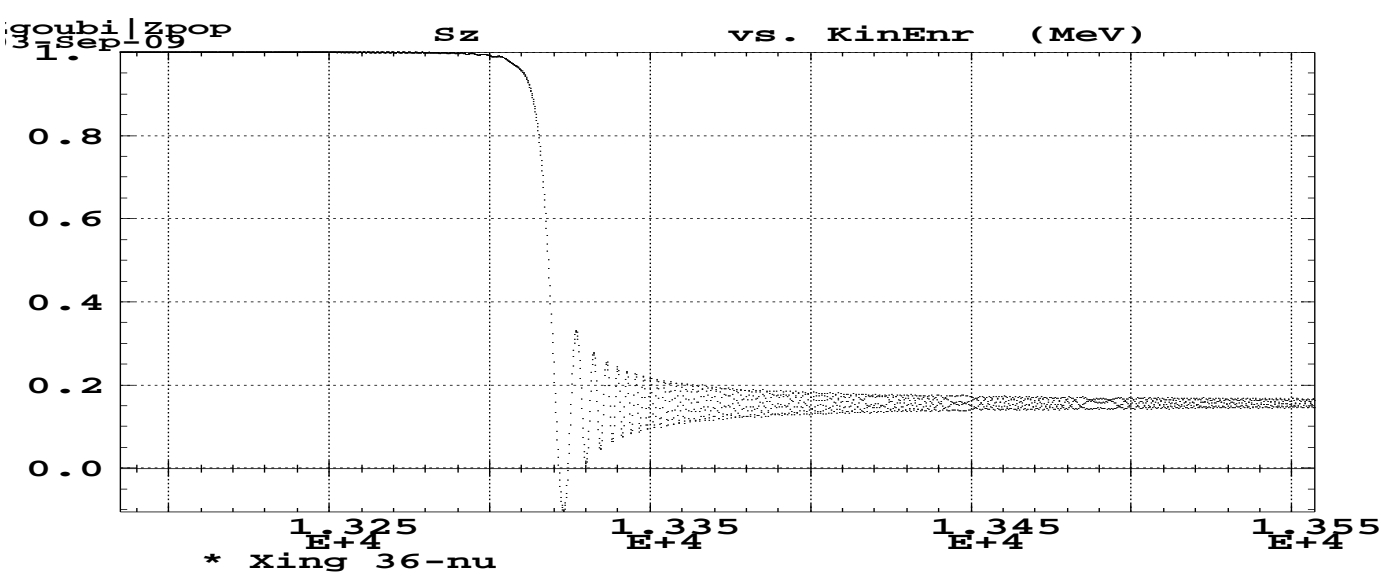

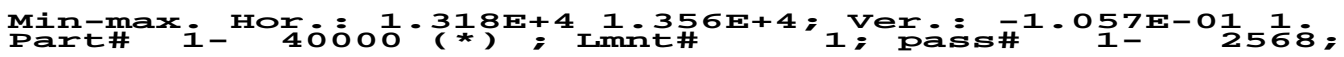

Figure 51: $S_{z}$ versus kinetic energy.

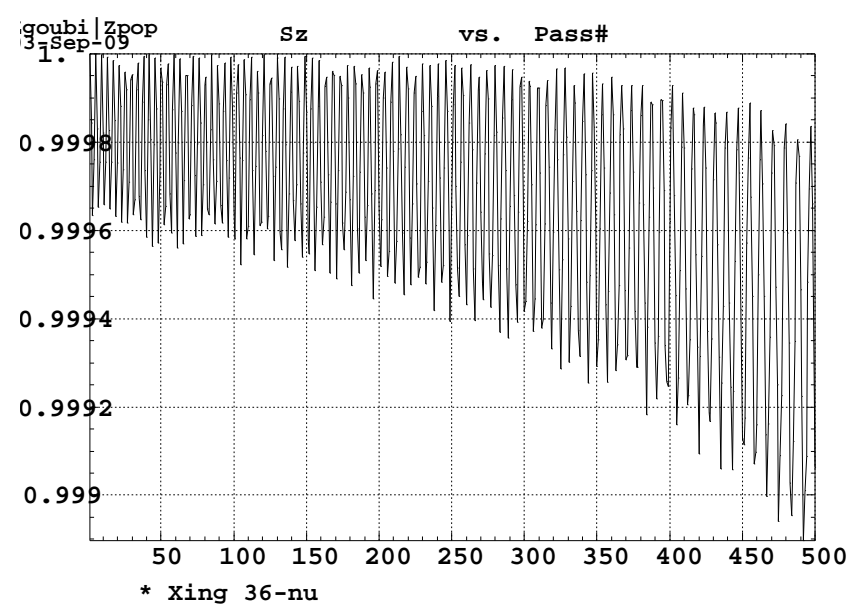

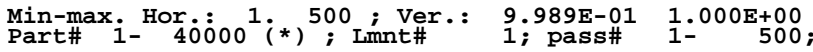

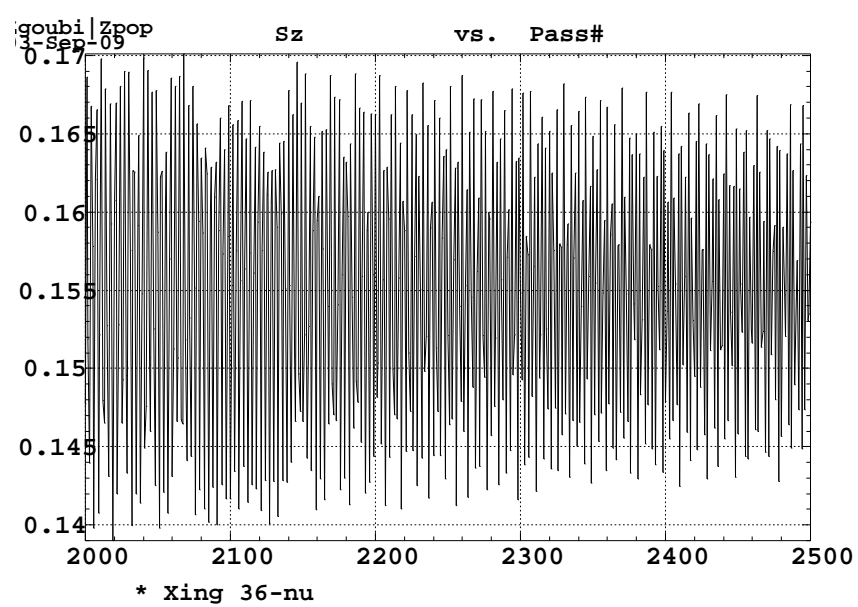

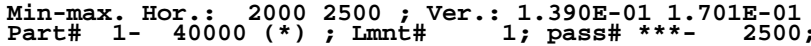

Figure 52: Zoom on initial $S_{z}$.

Figure 53: Zoom on final $S_{z}$.

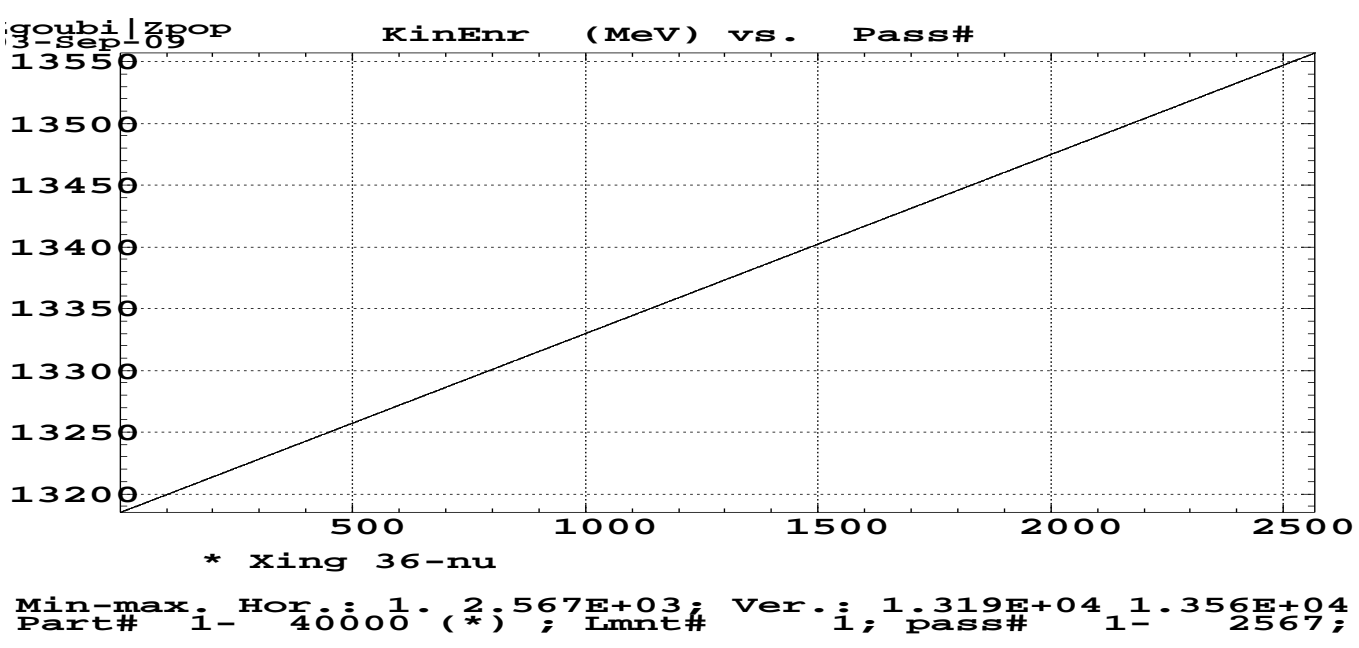

Figure 54: Kinetic E versus turn number. 


$$
\gamma G=36-\nu_{z}(\mathbf{1 3 . 3 1 5 7 5} \mathbf{G e V})
$$

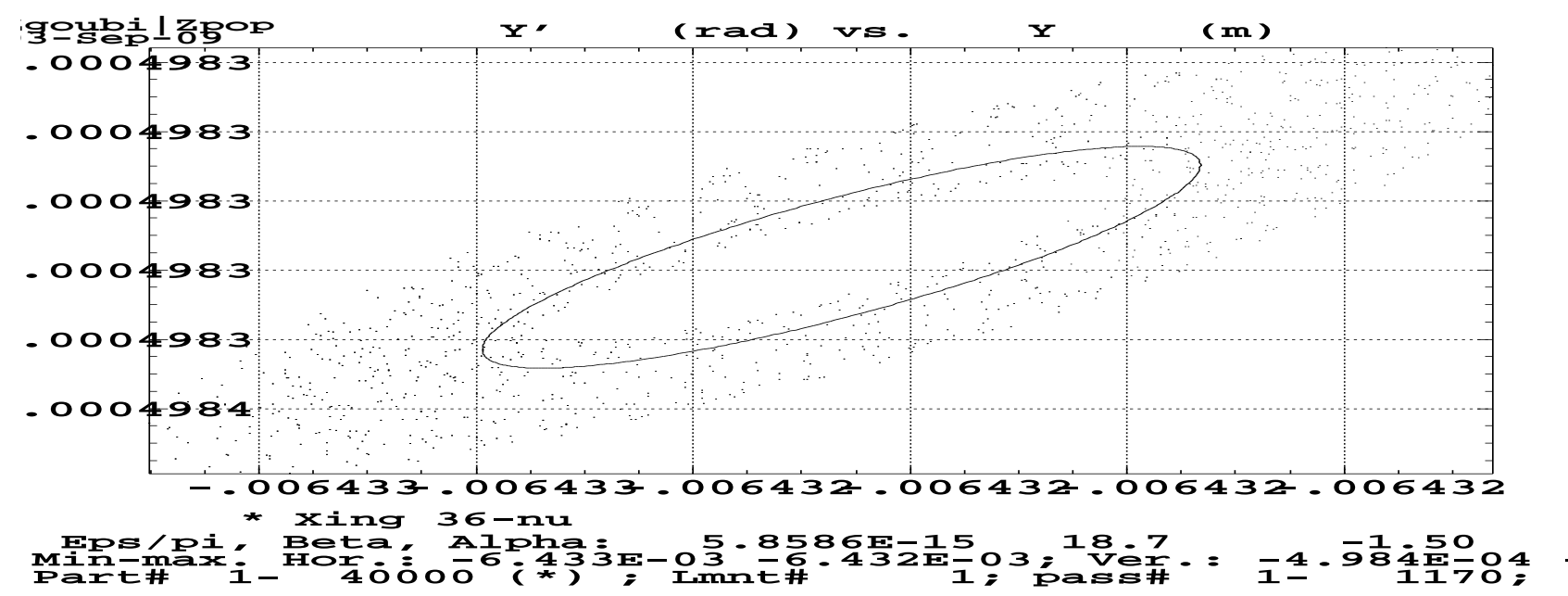

Figure 55: $x-x^{\prime}$.

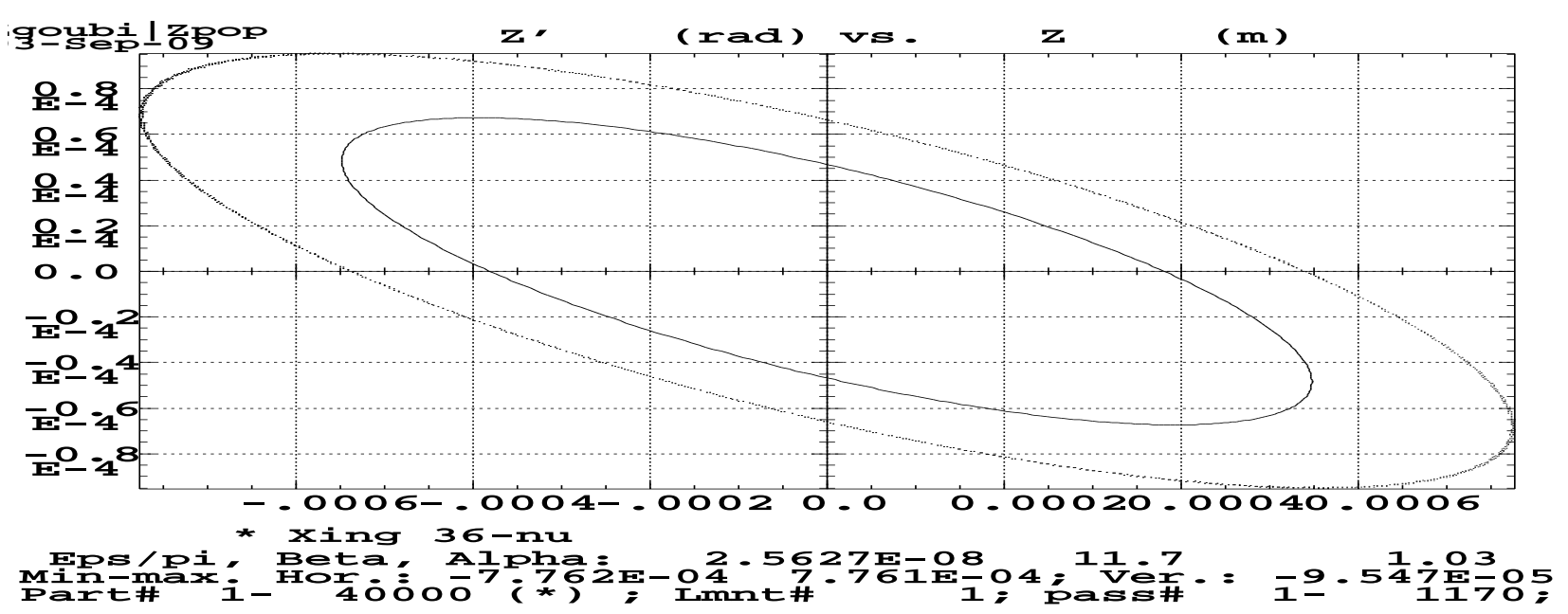

Figure 56: z-z'.

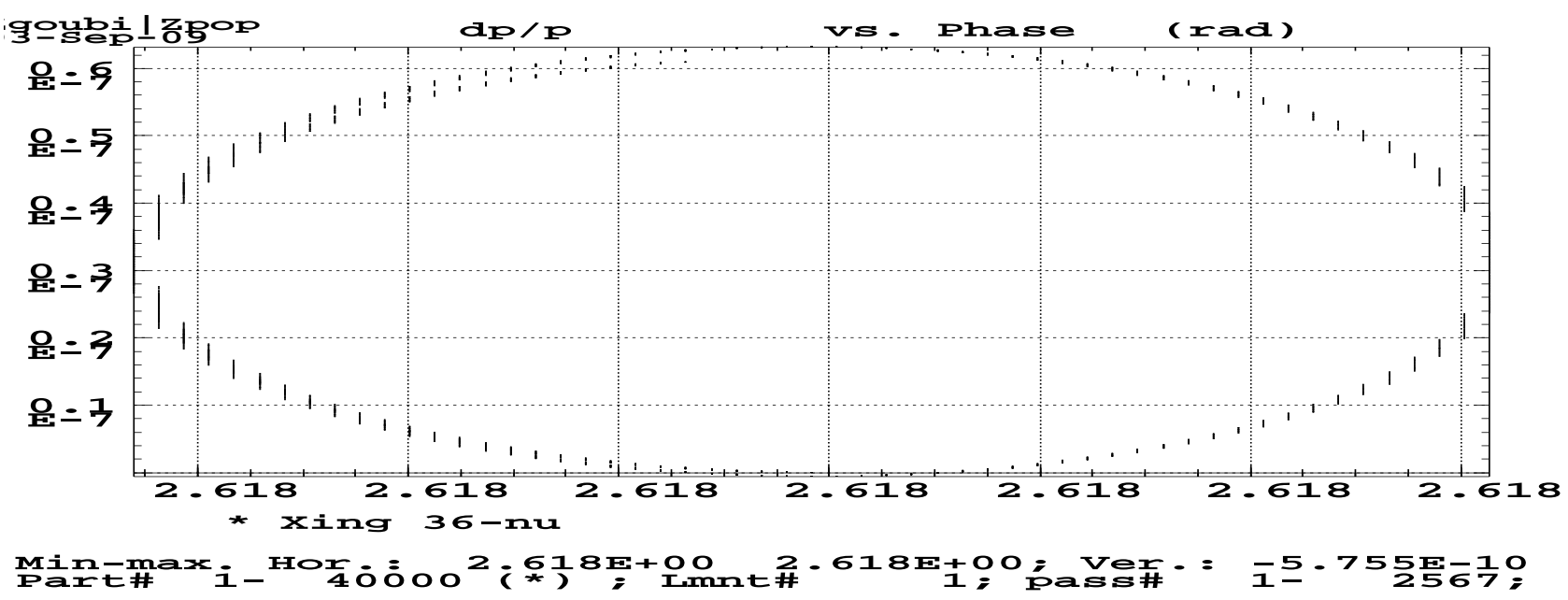

Figure 57: dp-phase. 


\subsection{7 $\gamma G=48-\nu_{z}(\mathbf{1 9 . 5 9 5 8 5} \mathbf{G e V})$}

\section{Tracking data}

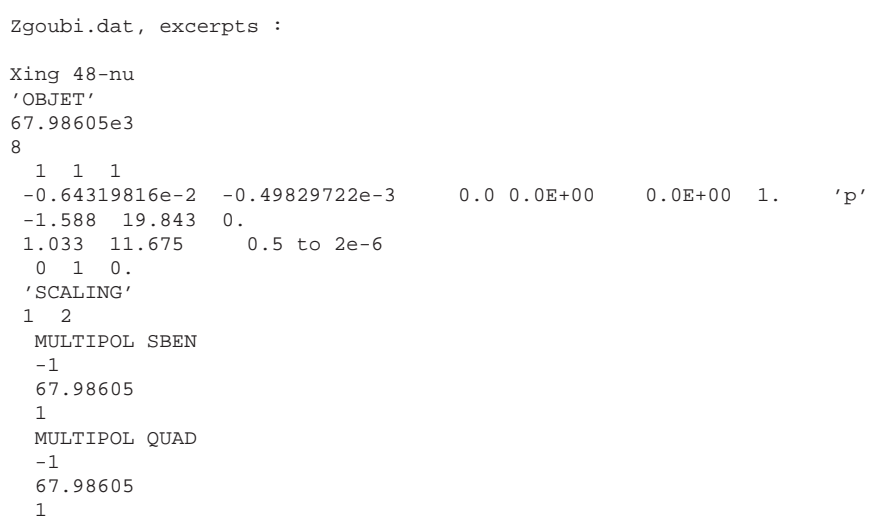

\section{Strength}

Eq. 2 yields the results displayed in Tab. 4 .

Table 4: Resonance strength for various vertical invariant values.

$\begin{array}{ccccccc}\epsilon_{z} / \pi & A^{2} & \left|J_{n}\right|^{2} & A^{2} / \epsilon_{z} / \pi & \left|J_{n}\right|^{2} / \epsilon_{z} / \pi & p_{\text {init }} & p_{\text {final }} \\ \left(\times 10^{-6}\right) & & & & & & \\ .125 & 0.04630575 & 1.2999233 \mathrm{E}-06 & 370446.0 & 10.39939 & 1 & 0.9095 \\ .25 & 0.09862635 & 2.7686992 \mathrm{E}-06 & 394505.4 & 11.07480 & 0.9998 & 0.812 \\ .5 & 0.1913054 & 5.3704412 \mathrm{E}-06 & 382610.8 & 10.74088 & 0.9996 & 0.6515 \\ 1 & 0.3895315 & 1.0935165 \mathrm{E}-05 & 389531.5 & 10.93517 & 0.9993 & 0.3545 \\ 2 & 0.8073937 & 2.2665648 \mathrm{E}-05 & 403696.8 & 11.33282 & 0.9985 & -0.1078\end{array}$


$\gamma G=48-\nu_{z}(19.59585 \mathrm{GeV})-\epsilon_{z} / \pi=0.12510^{-6}$

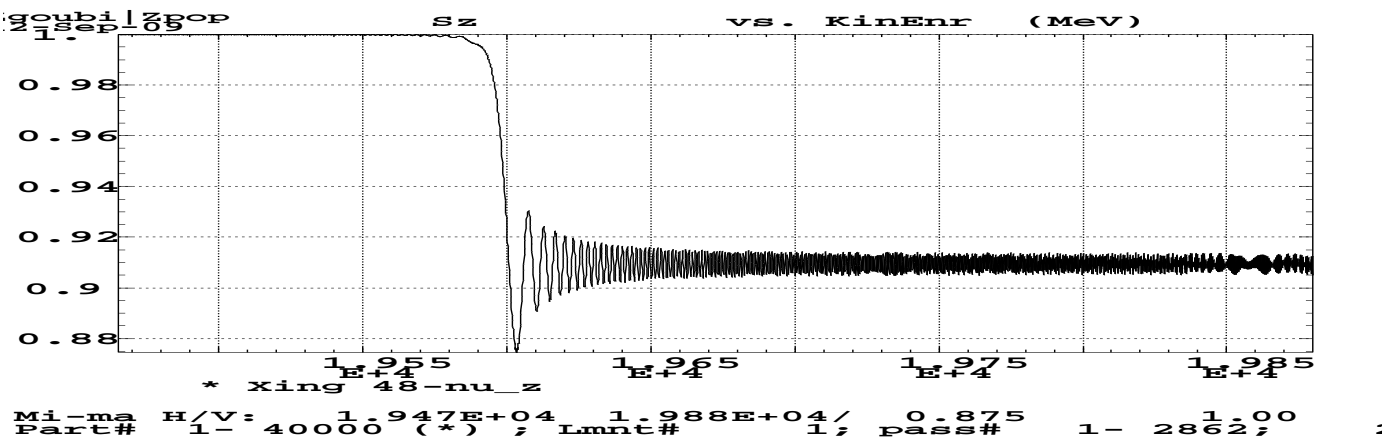

Figure 58: $S_{z}$ versus kinetic energy.

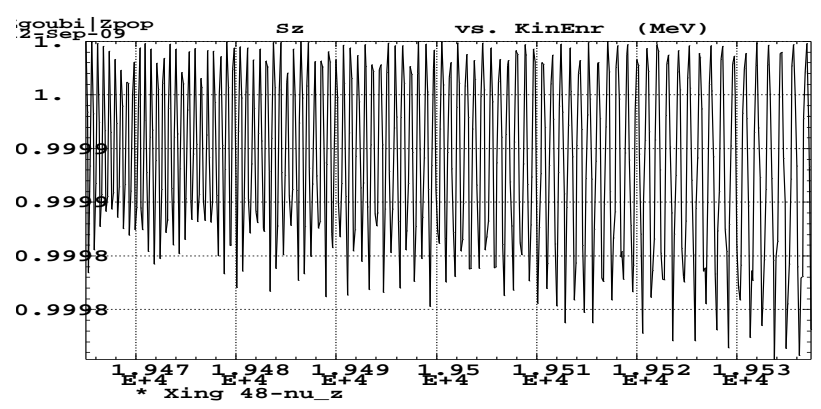

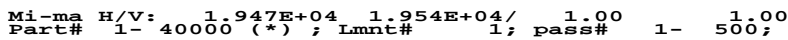

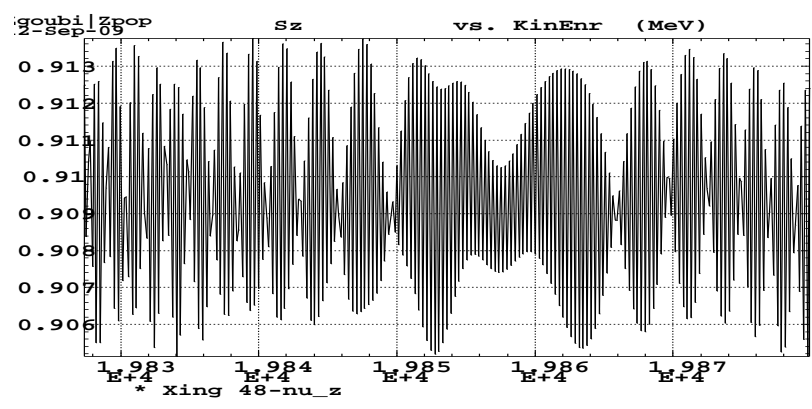

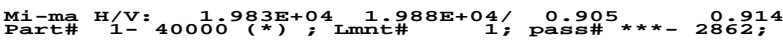

Figure 59: Zoom on initial $S_{z}$.

Figure 60: Zoom on final $S_{z}$.
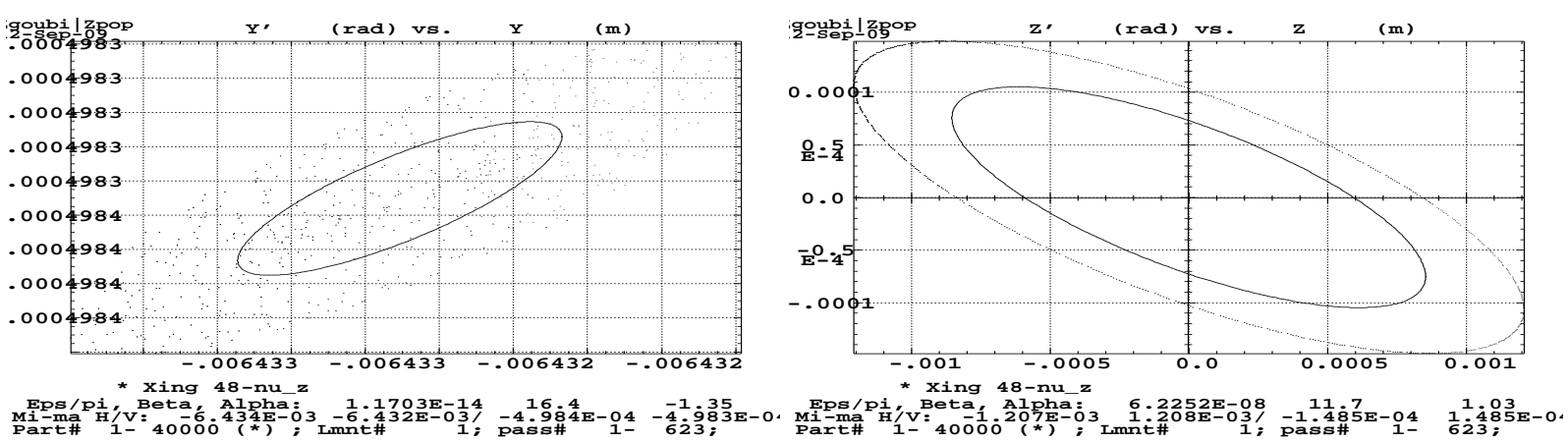

Figure 61: $x-x^{\prime}$ and z-z'.

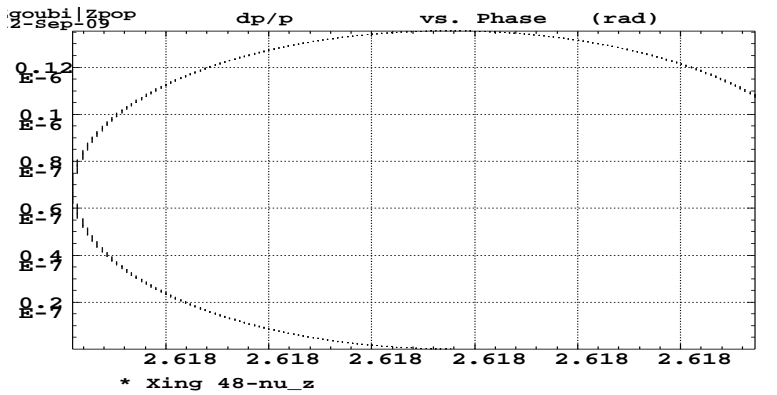

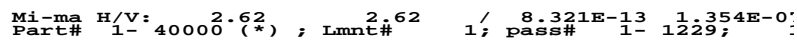

Figure 62: dp-phase (1350 turns). 
$\gamma G=48-\nu_{z}(19.59585 \mathrm{GeV})-\epsilon_{z} / \pi=0.2510^{-6}$

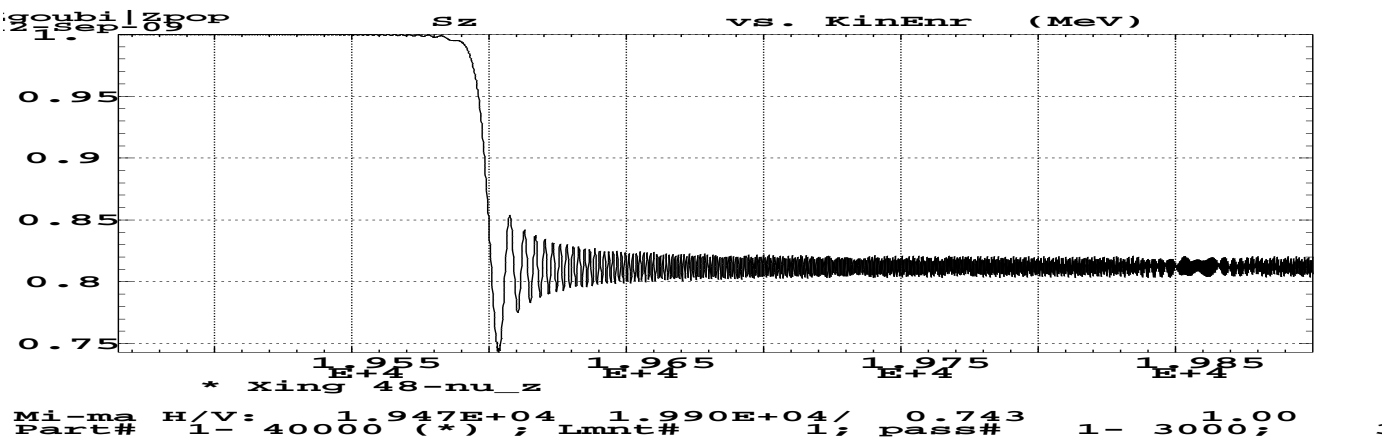

Figure 63: $S_{z}$ versus kinetic energy.

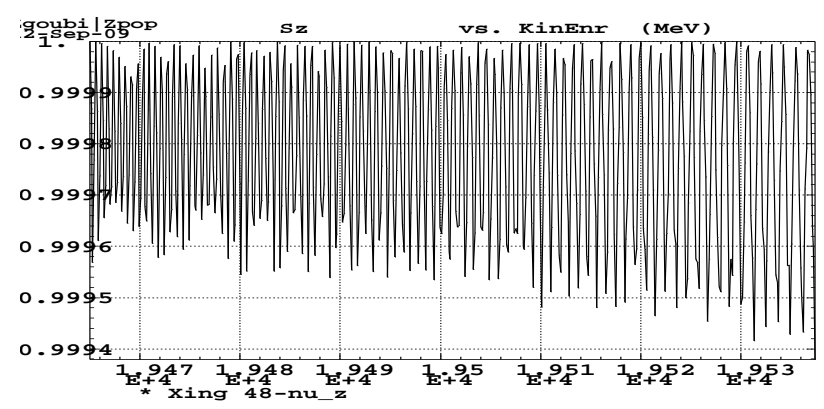

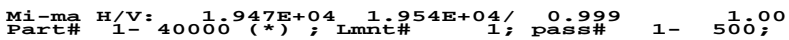

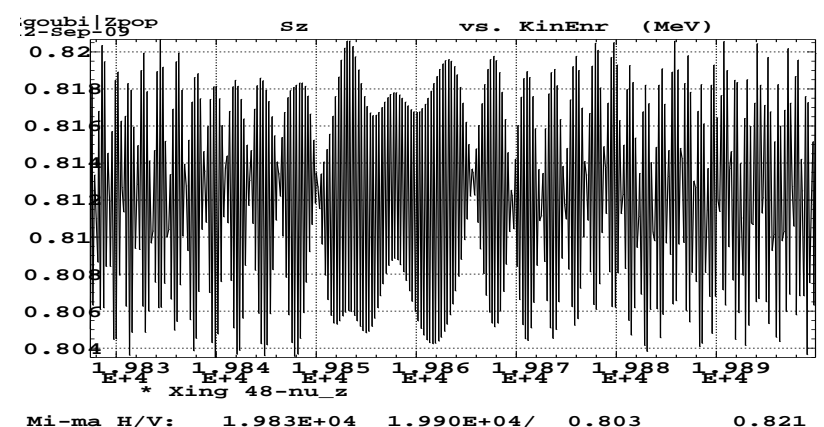

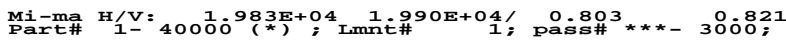

Figure 64: Zoom on initial $S_{z}$.

Figure 65: Zoom on final $S_{z}$.
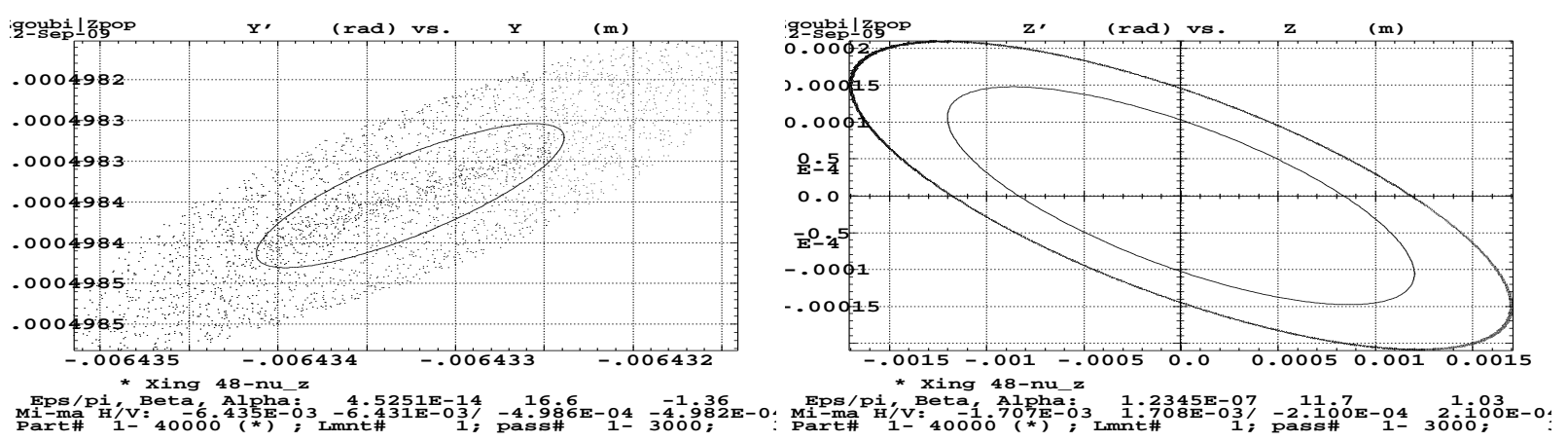

Figure 66: $x-x^{\prime}$ and $z-z^{\prime}$.

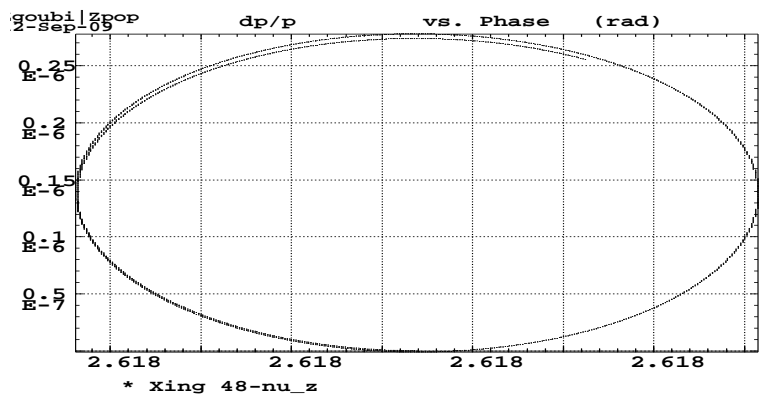

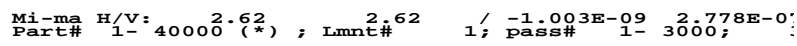

Figure 67: dp-phase. 
$\gamma G=48-\nu_{z}(19.59585 \mathrm{GeV})-\epsilon_{z} / \pi=0.510^{-6}$

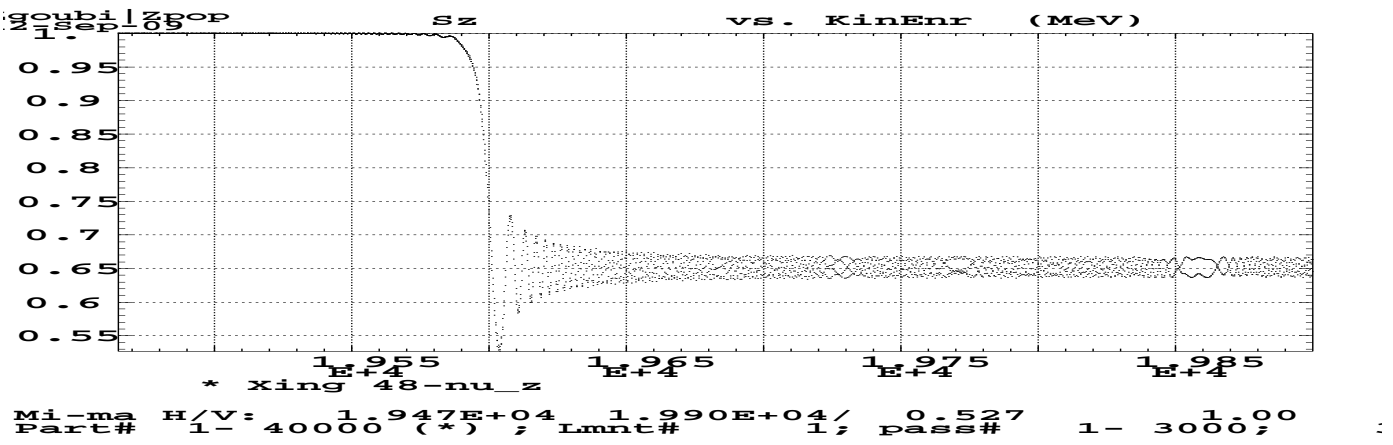

Figure 68: $S_{z}$ versus kinetic energy.

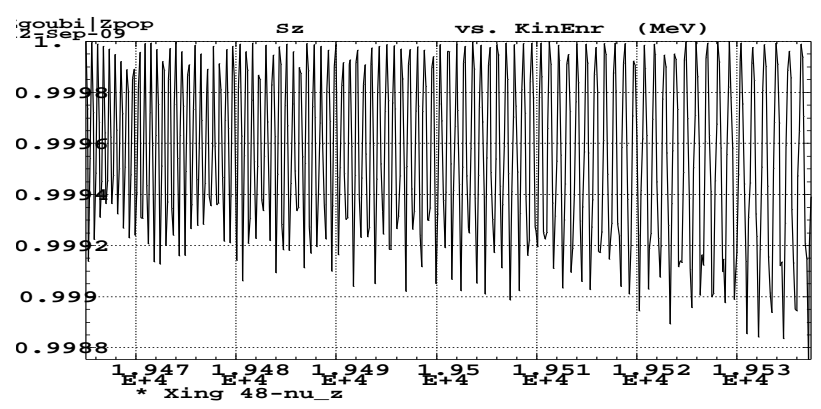

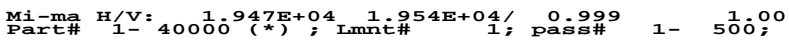

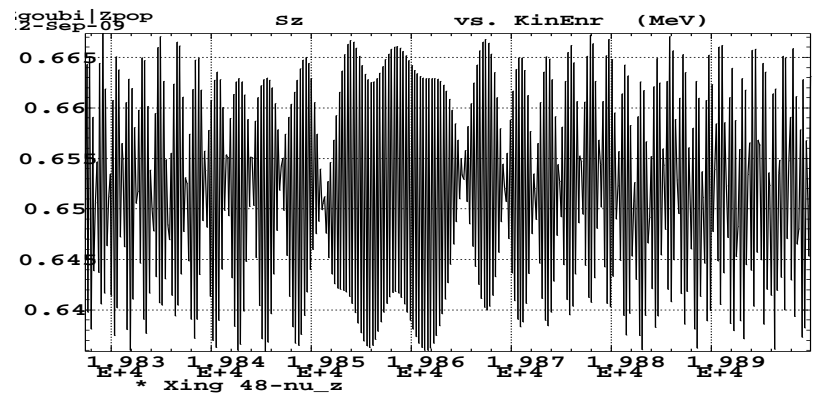

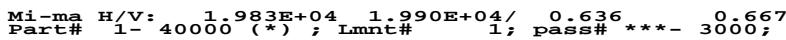

Figure 69: Zoom on initial $S_{z}$.

Figure 70: Zoom on final $S_{z}$.
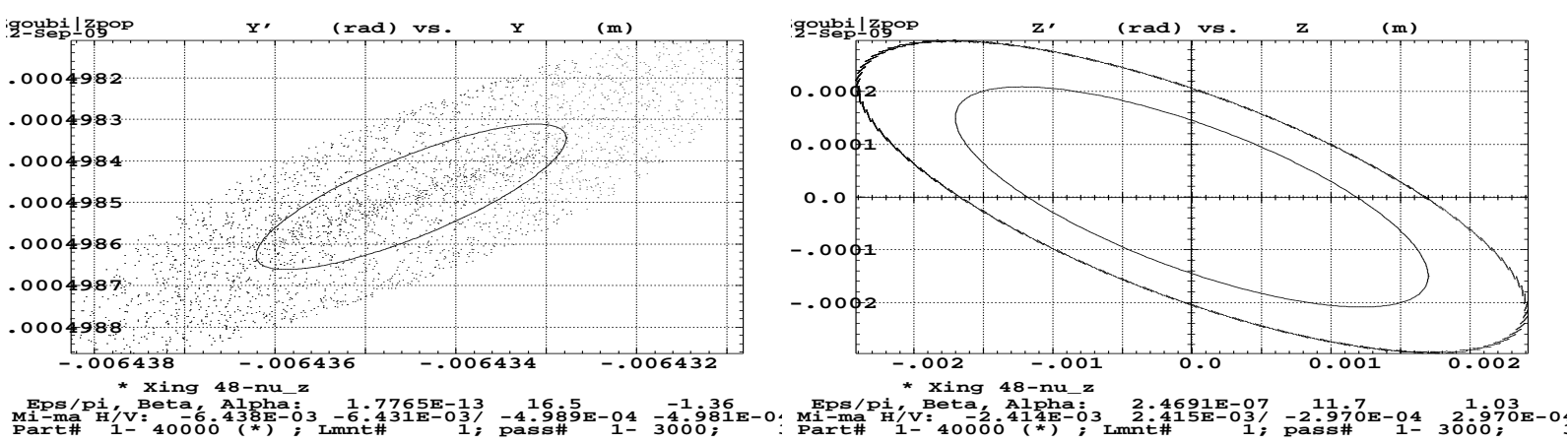

Figure 71: $\mathrm{x}-\mathrm{x}^{\prime}$ and $\mathrm{z}-\mathrm{z}^{\prime}$.

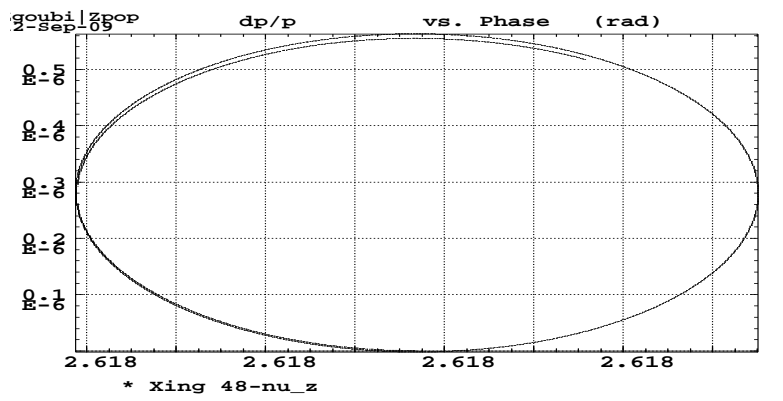

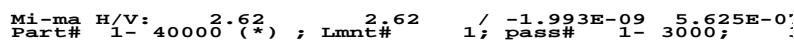

Figure 72: dp-phase. 
$\gamma G=48-\nu_{z}(19.59585 \mathrm{GeV})-\epsilon_{z} / \pi=10^{-6}$

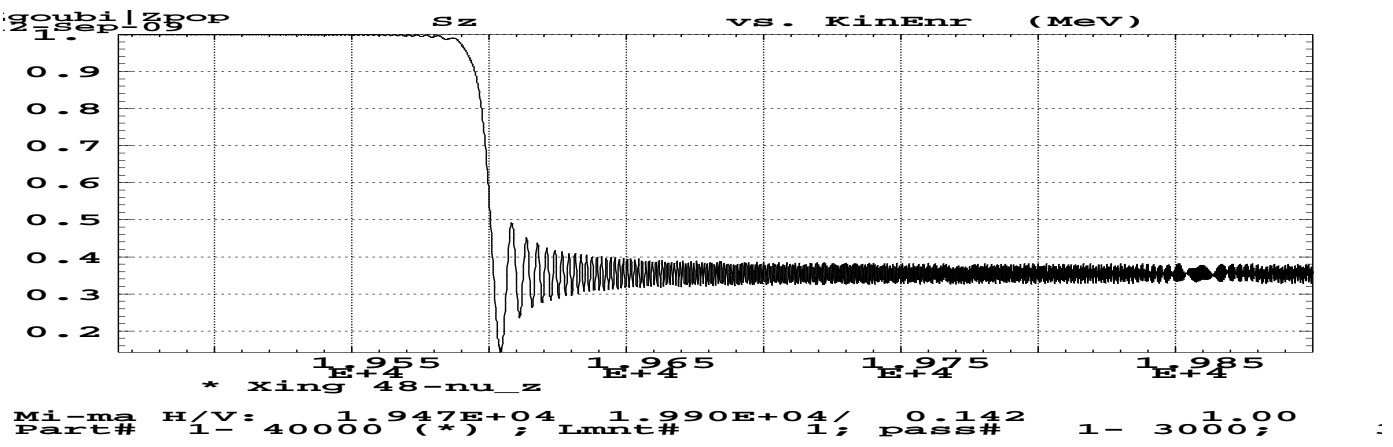

Figure 73: $S_{z}$ versus kinetic energy.

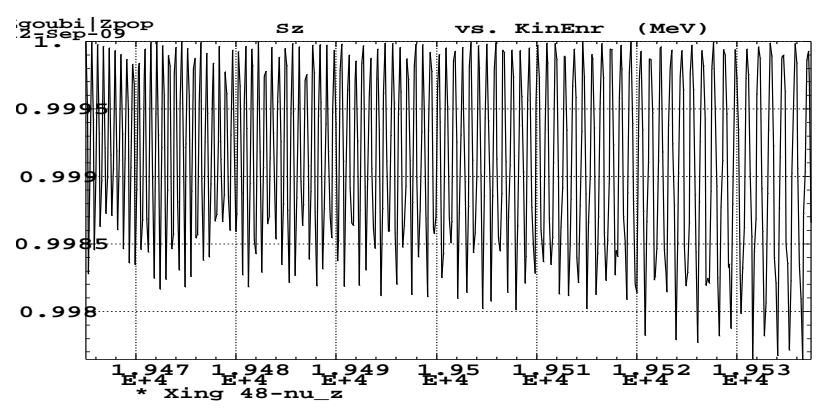

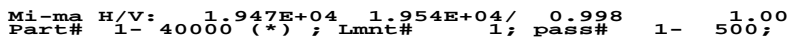

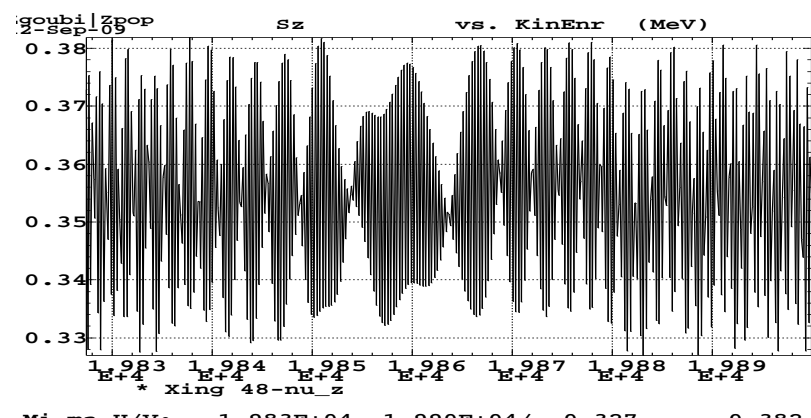

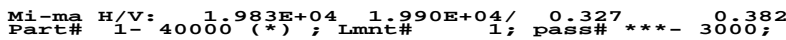

Figure 74: Zoom on initial $S_{z}$.

Figure 75: Zoom on final $S_{z}$.
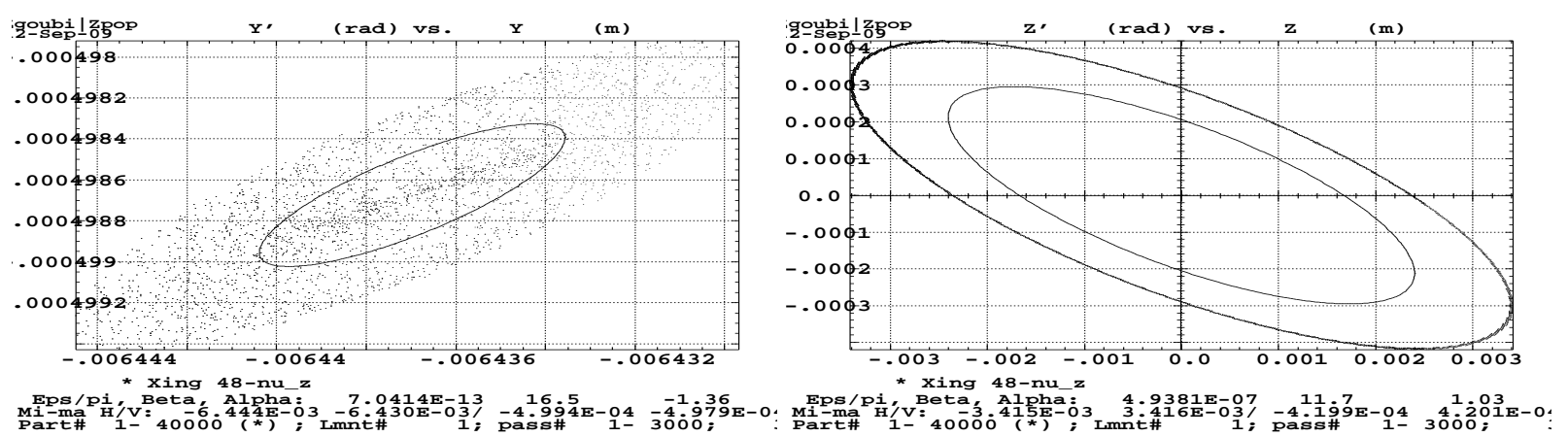

Figure 76: $x-x^{\prime}$ and $z-z^{\prime}$.

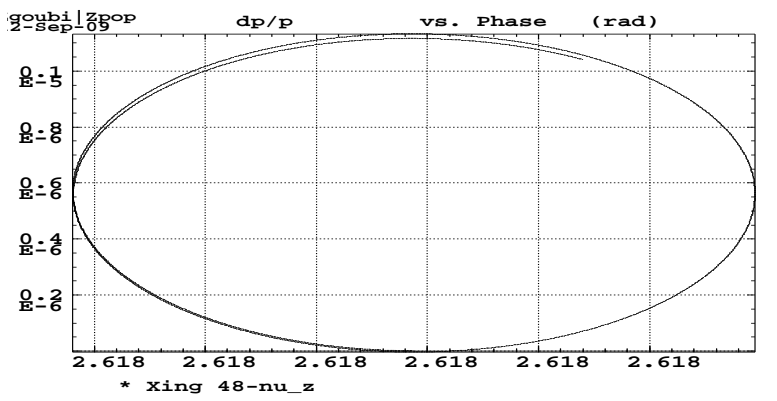

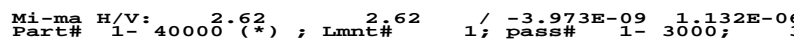

Figure 77: dp-phase. 
$\gamma G=48-\nu_{z}(19.59585 \mathrm{GeV})-\epsilon_{z} / \pi=210^{-6}$

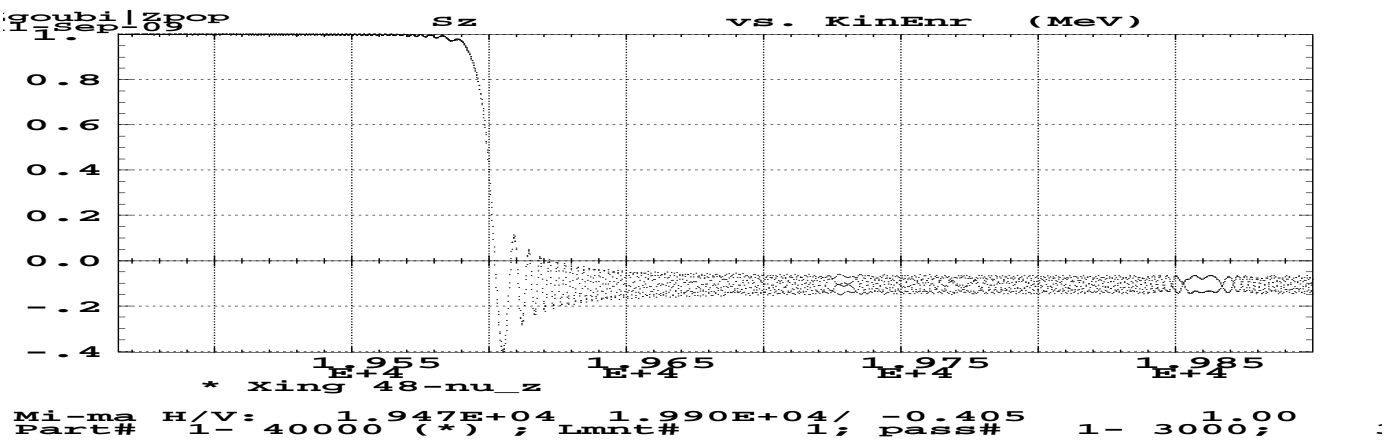

Figure 78: $S_{z}$ versus kinetic energy.

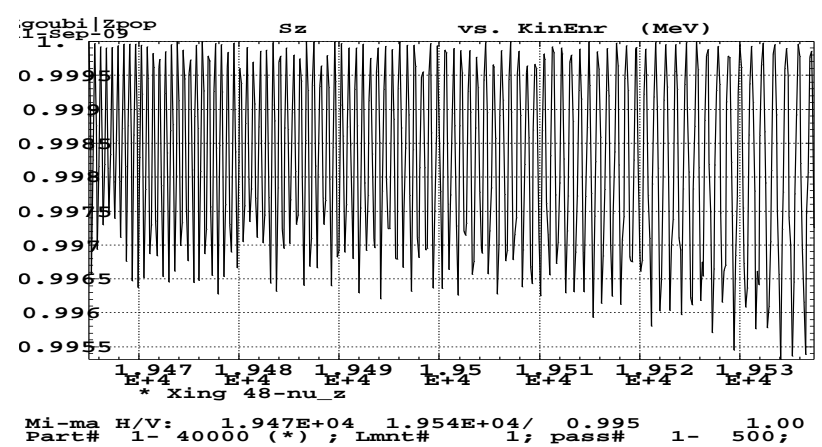

Figure 79: Zoom on initial $S_{z}$.

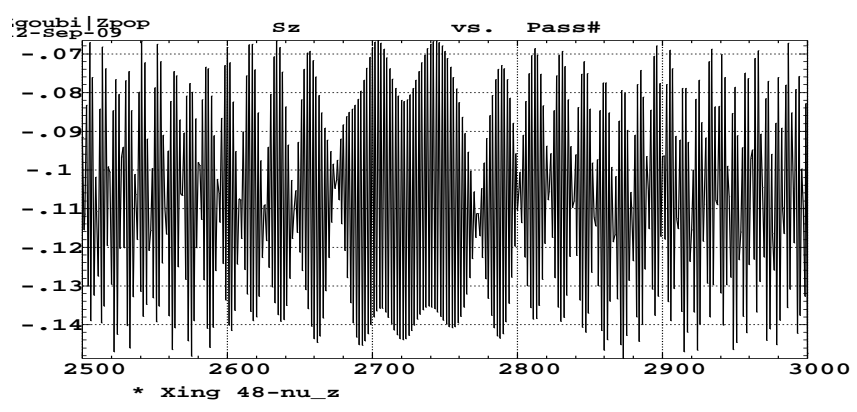

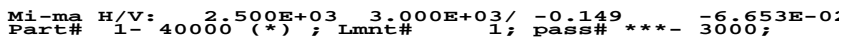

Figure 80: Zoom on final $S_{z}$.
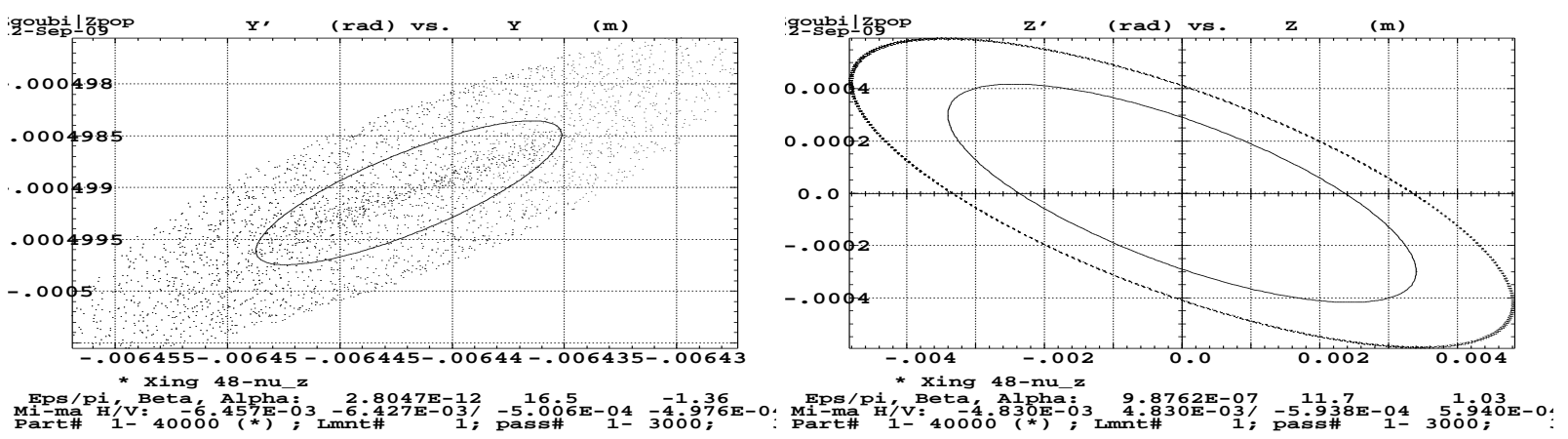

Figure 81: $\mathrm{x}-\mathrm{x}^{\prime}$ and $\mathrm{z}-\mathrm{z}^{\prime}$.

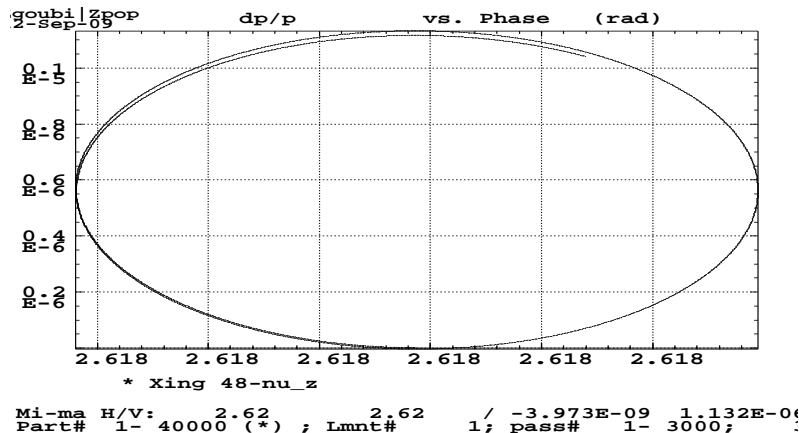

Figure 82: dp-phase. 


\subsection{8 $\gamma G=48-\nu_{z}$, bare lattice (all bends' sextu off, all quads off)}

Resonance strength computed from Zgoubi tracking differs from DEPOL's results in some cases of weak resonances, such as $\gamma G=48-\nu_{z}$. For that reason, in order to assess possible effect of sextupoles, we switch-off all sextupole components in all main bends. We also switch-off all tuning quadrupoles, the focusing is therefore due to the sole main bends' quadrupole component.

The following shows that the initial results are practically unchanged, the same resonance strength comes out of the ray-tracing.

Setting all sextupoles and quadrupoles off does not change much the ring parameters, 0 -th and 1-st order machine parameters take the values given in Tab. 5. Earlier values are recalled for comparison.

A particle with vertical emittance $\epsilon_{y} / \pi=10^{-6} \mathrm{~m}$.rad is launched through the resonance. Spin motion upon crossing does not change much either, this can be observed from Fig. 83, by comparison with Fig. 58, page 42.

Table 5: Bare lattice parameters. Old ones (copied from Tab. 1) are recalled in the right column for comparison.

\begin{tabular}{lccc} 
& & New parameters & Old parameters \\
Reference momentum & (relative) & 1 & 1 \\
Circumference & (m) & 807.0437 & 807.0438 \\
Qx, Qy & & $0.71066,0.76412$ & $0.71195,0.76346$ \\
$\begin{array}{l}\text { Periodic functions at "Begin AGS" : } \\
\beta_{x}, \beta_{y}\end{array}$ & & & \\
$\alpha_{x}, \alpha_{y}$ & $(\mathrm{~m})$ & $19.7850,11.7005$ & $19.8432,11.6751$ \\
$D_{x}, D_{x}^{\prime}$ & & $-1.585,-1.037$ & $-1.588,1.033$ \\
closed orbit, $x_{c o}, x_{c o}^{\prime}$ & $(\mathrm{m},-)$ & $2.043,0.144$ & $2.034,0.144$ \\
& $\mathrm{~mm}, \mathrm{mrad}$ & $-0.645,-0.50$ & $-6.43-0.50^{1,3}$ \\
$\gamma G=48-\nu_{z}$ & & & \\
kinetic energy on resonance & $(\mathrm{GeV})$ & 19.6097 & 39.236 \\
$B \rho$ on resonance & $(\mathrm{T}) \mathrm{m})$ & 68.4692 & 68.5958 \\
& & & \\
\hline
\end{tabular}

\section{Tracking data}

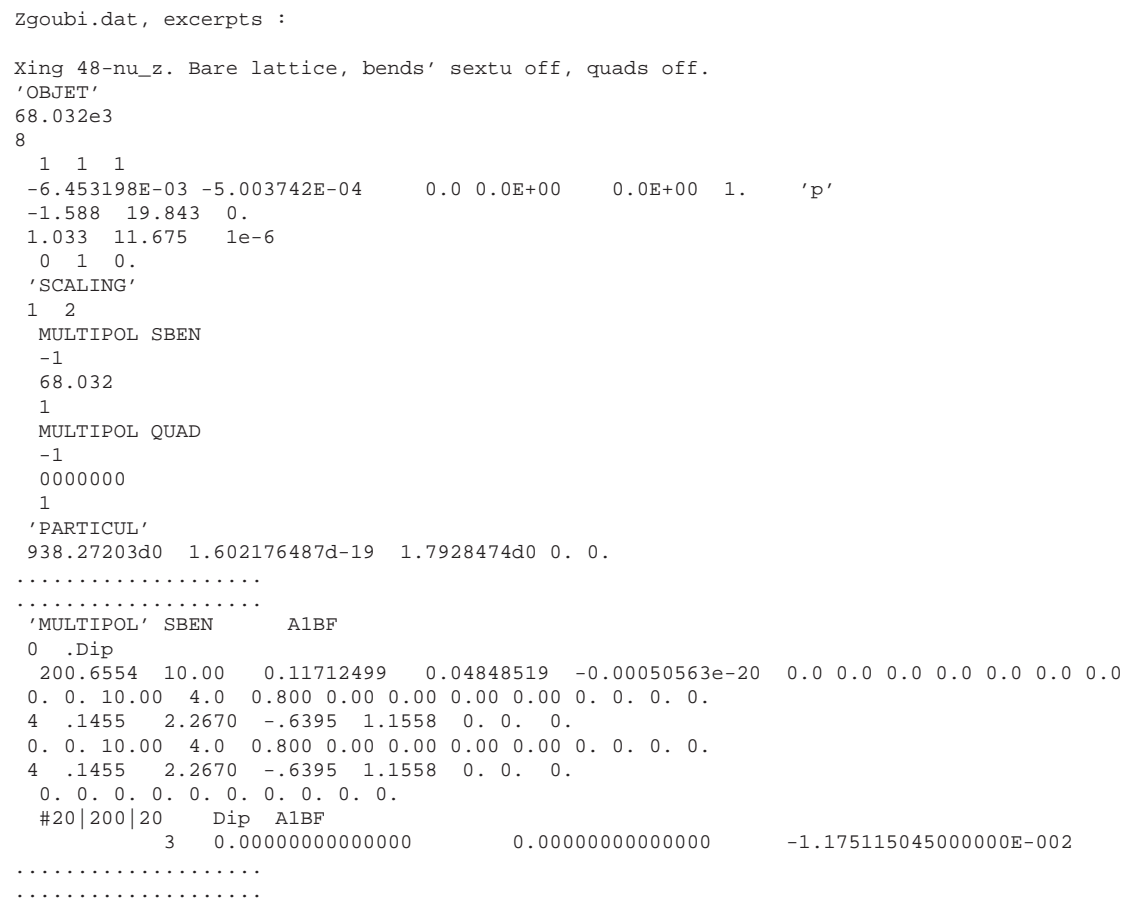


$\gamma G=48-\nu_{z}(19.6097 \mathrm{GeV})-\epsilon_{z} / \pi=10^{-6}$

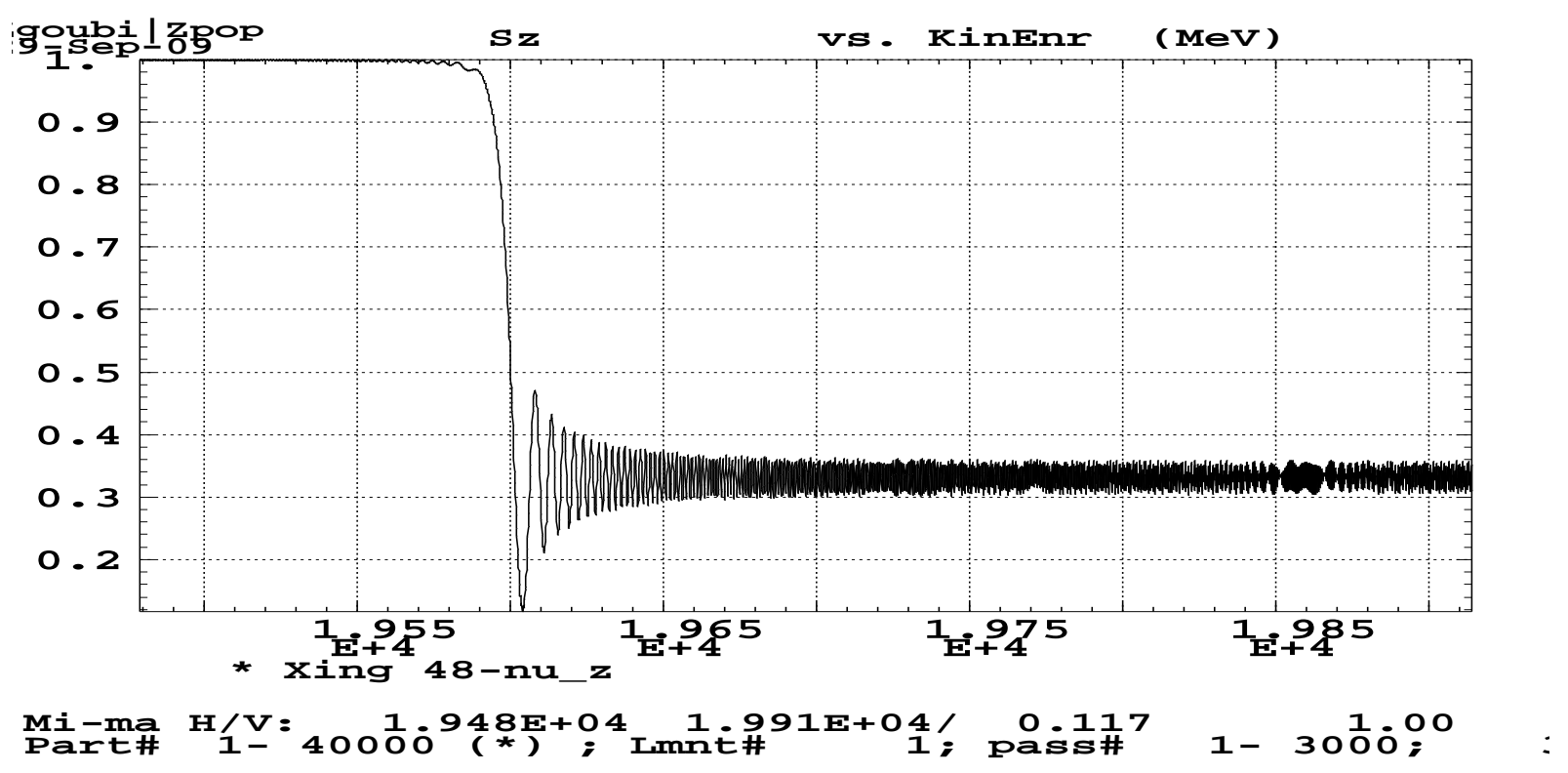

Figure 83: $S_{z}$ versus kinetic energy. See Fig. 58, page 42 for comparison. 


\subsection{9 $\gamma G=36+\nu_{z}(\mathbf{2 2 . 4 8 8 3 2} \mathbf{~ G e V})$}

\section{Tracking data}

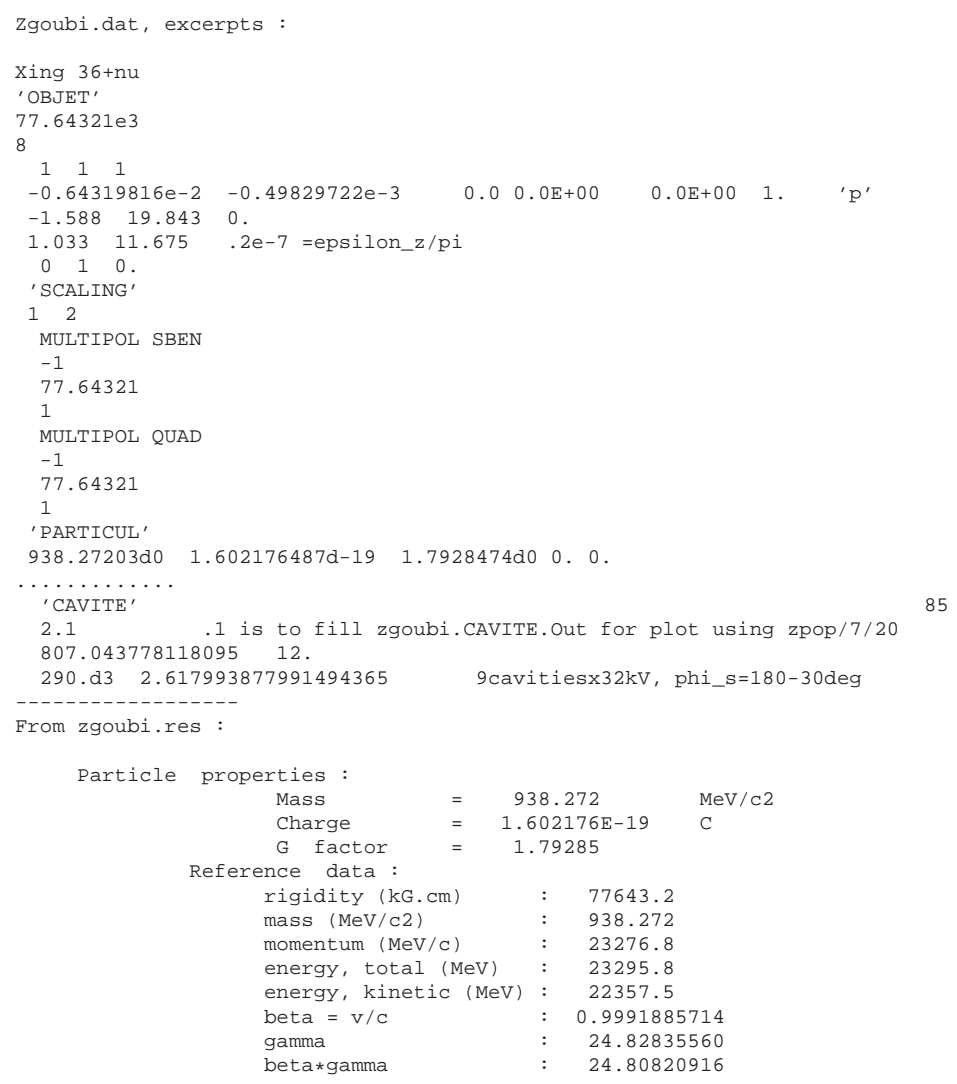

\section{Strengths}

From Figs. 20, 20 one gets

$\begin{array}{ccccccc}\epsilon_{z} / \pi & A^{2} & \left|J_{n}\right|^{2} & A^{2} / \epsilon_{z} / \pi & \left|J_{n}\right|^{2} / \epsilon_{z} / \pi & p_{\text {init }} & p_{\text {final }} \\ \left(10^{-6}\right) & & \left(10^{-5}\right) & & & & \\ 0.002 & 0.1329602 & 0.37325392 & 6.648009 \mathrm{E}+07 & 1866 & 1 . & 0.751 \\ 0.02 & 1.431838 & 4.0195435 & 7.1591912 \mathrm{E}+07 & 2009 & 1 . & -0.522\end{array}$


$\gamma G=36+\nu_{z}(22.48832 \mathrm{GeV})-\epsilon_{z} / \pi=0.00210^{-6}$

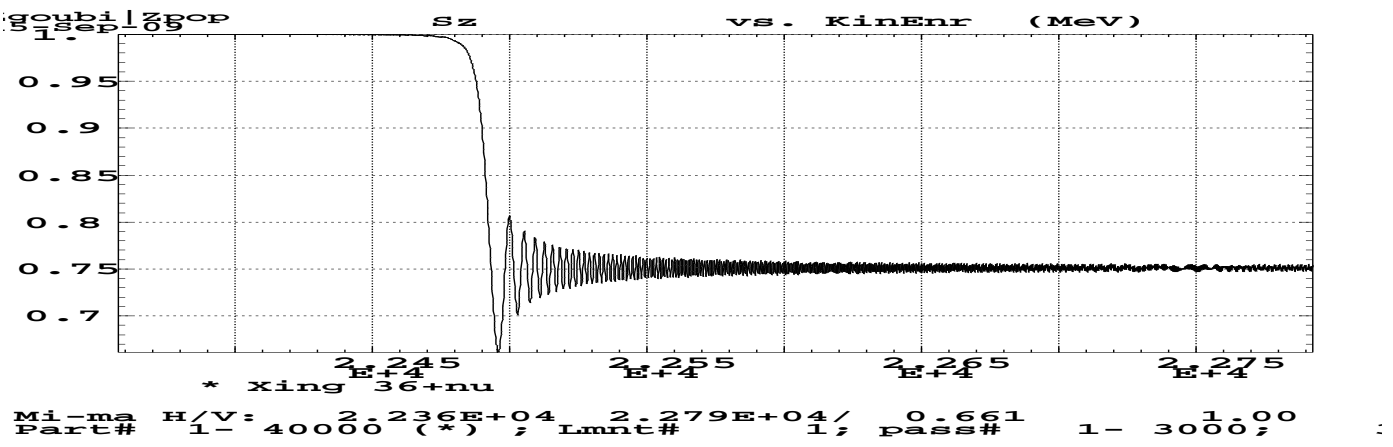

Figure 84: $S_{z}$ versus kinetic energy.

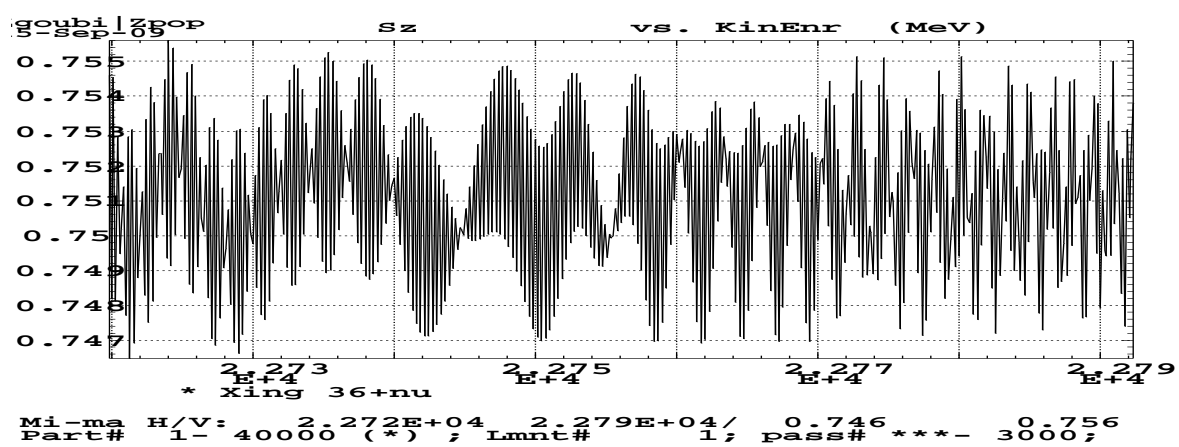

Figure 85: Zoom on final $S_{z}$.
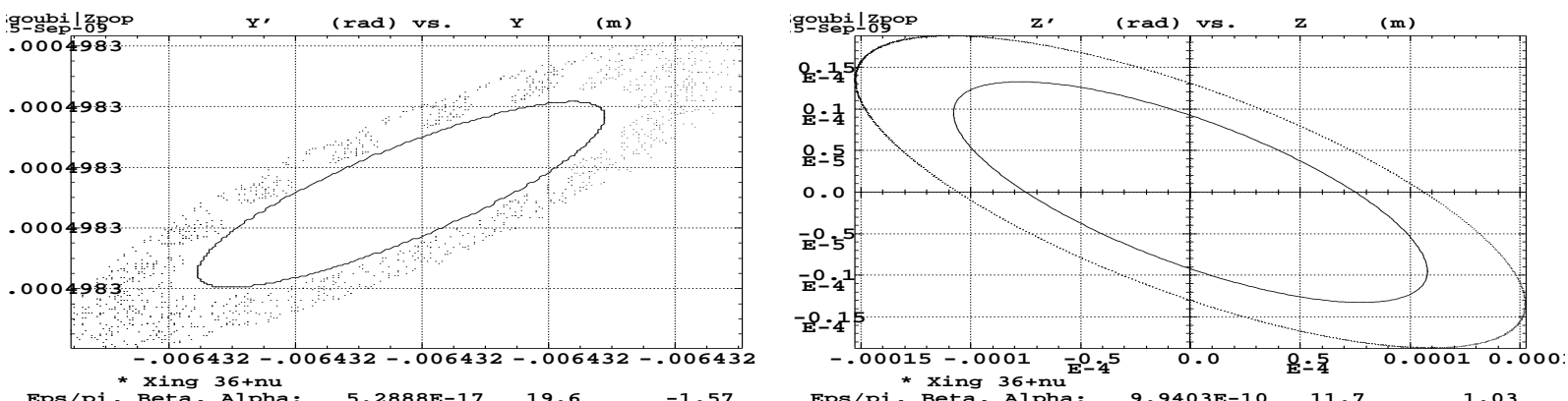

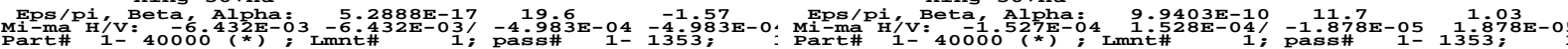

Figure 86: $x-x^{\prime}$ and z-z'.

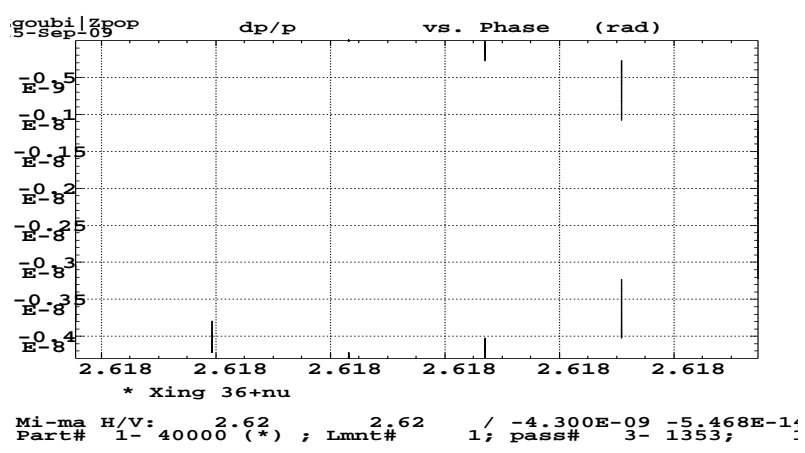

Figure 87: dp-phase. 
$\gamma G=36+\nu_{z}(22.48832 \mathrm{GeV})-\epsilon_{z} / \pi=0.0210^{-6}$

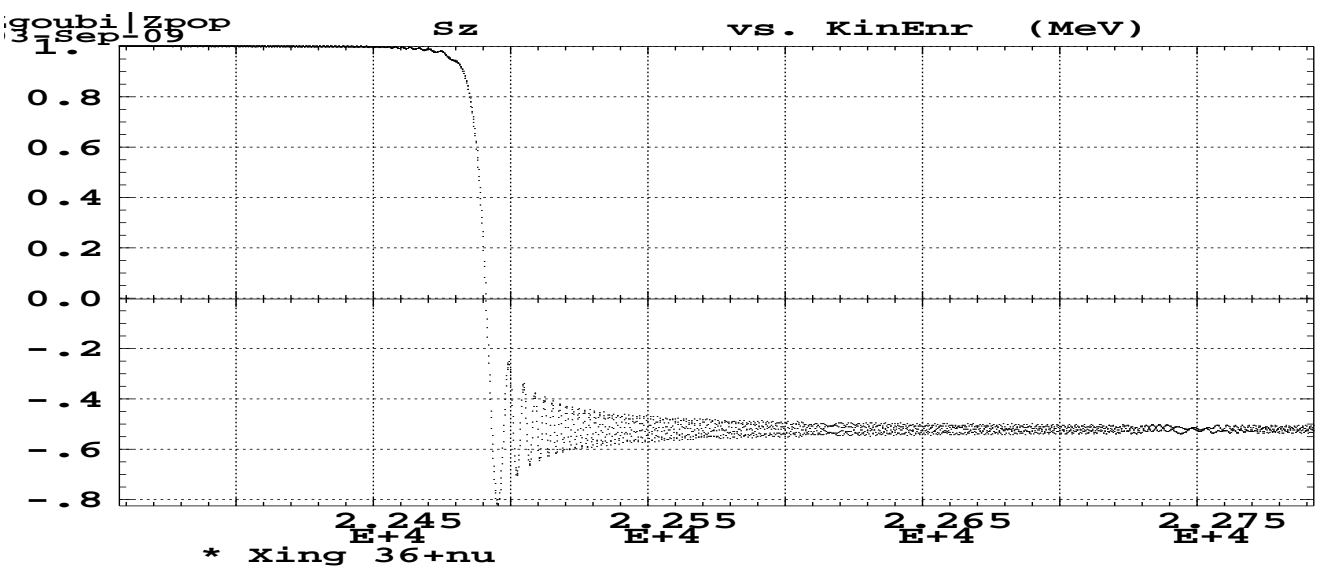

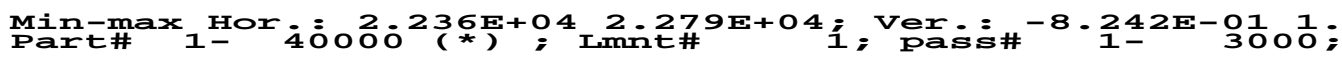

Figure 88: $S_{z}$ versus kinetic energy.

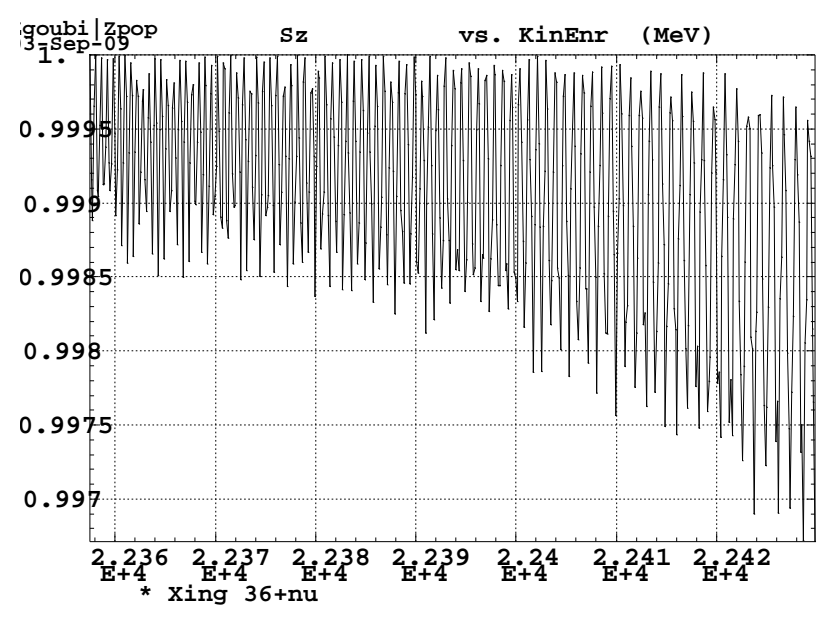

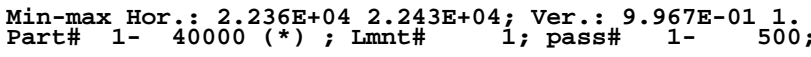

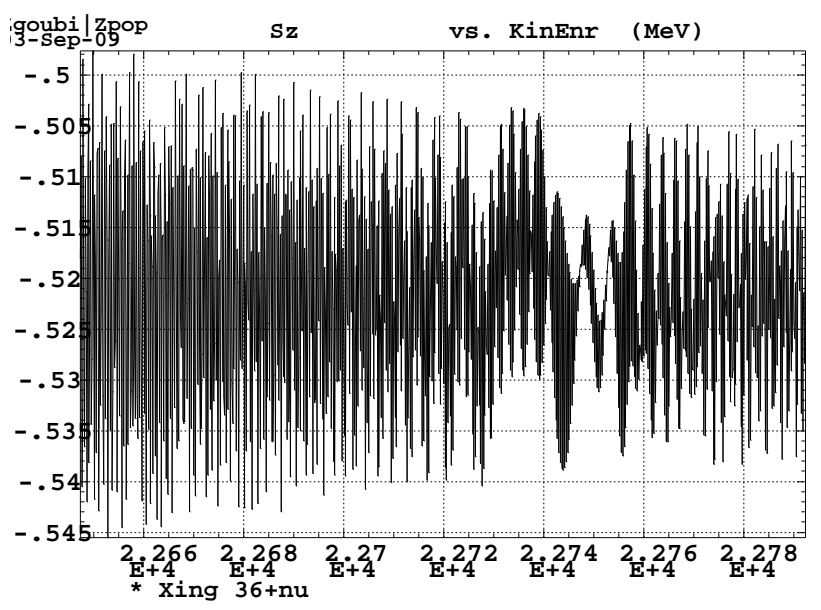

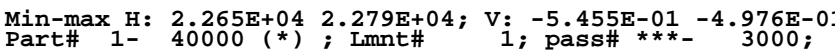

Figure 89: Zoom on initial $S_{z}$.

Figure 90: Zoom on final $S_{z}$.

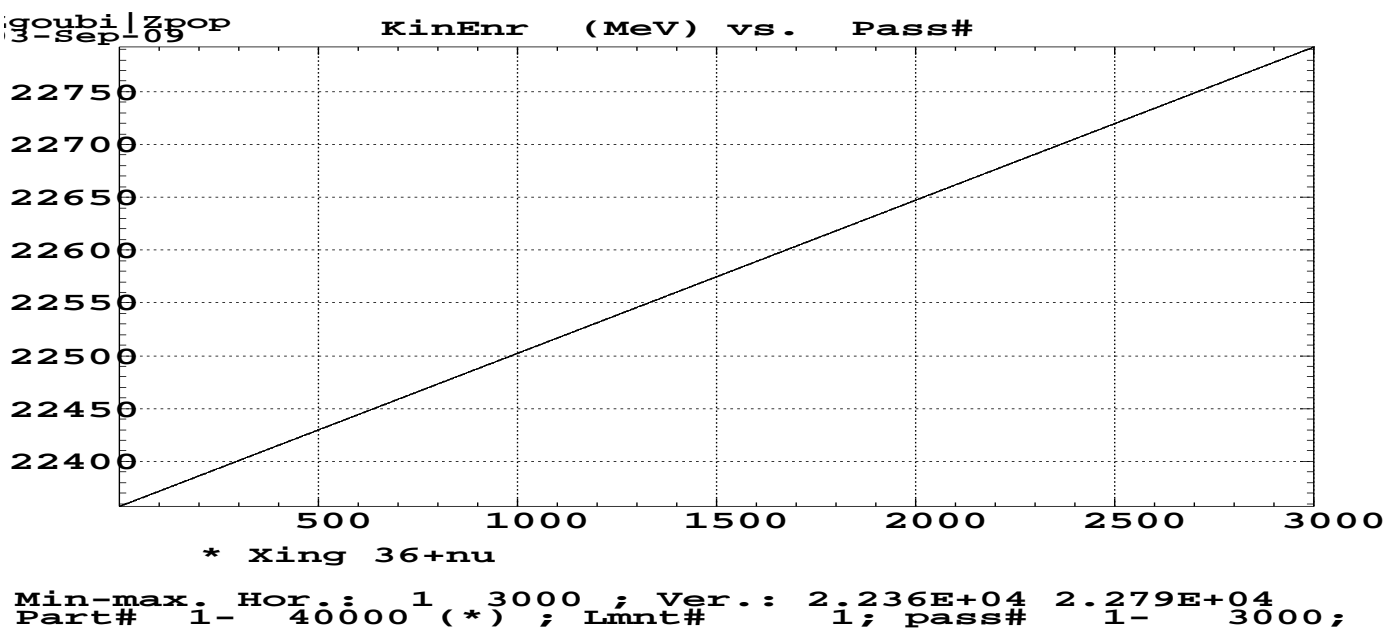

Figure 91: Kinetic E versus turn number. 
$\gamma G=36+\nu_{z}(22.48832 \mathrm{GeV})-\epsilon_{z} / \pi=0.0210^{-6}$

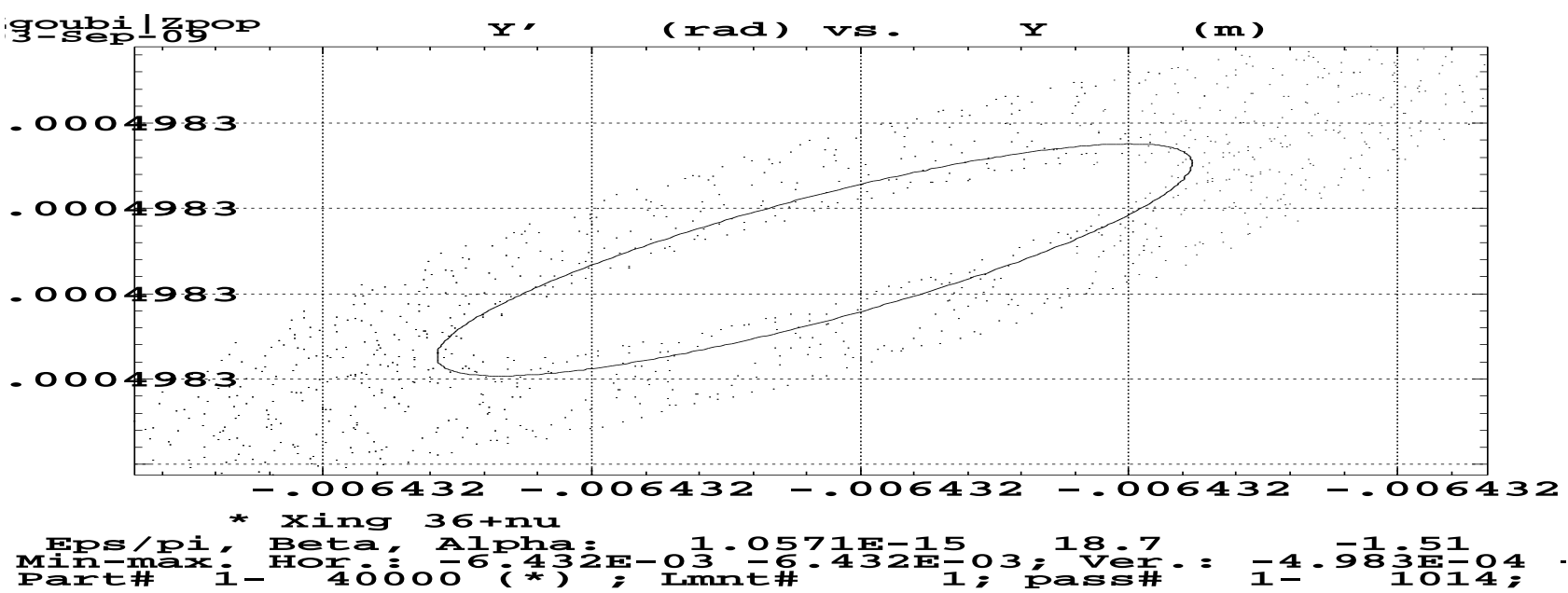

Figure 92: $x-x^{\prime}$.

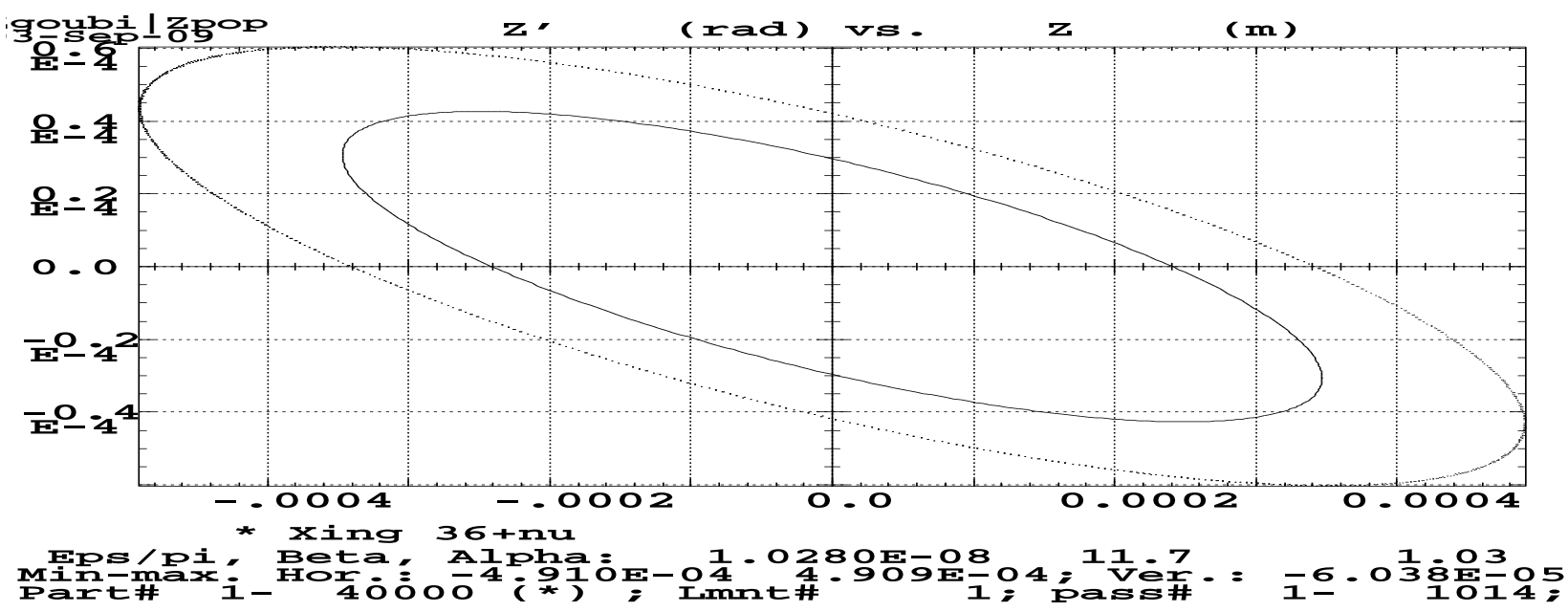

Figure 93: z-z'.

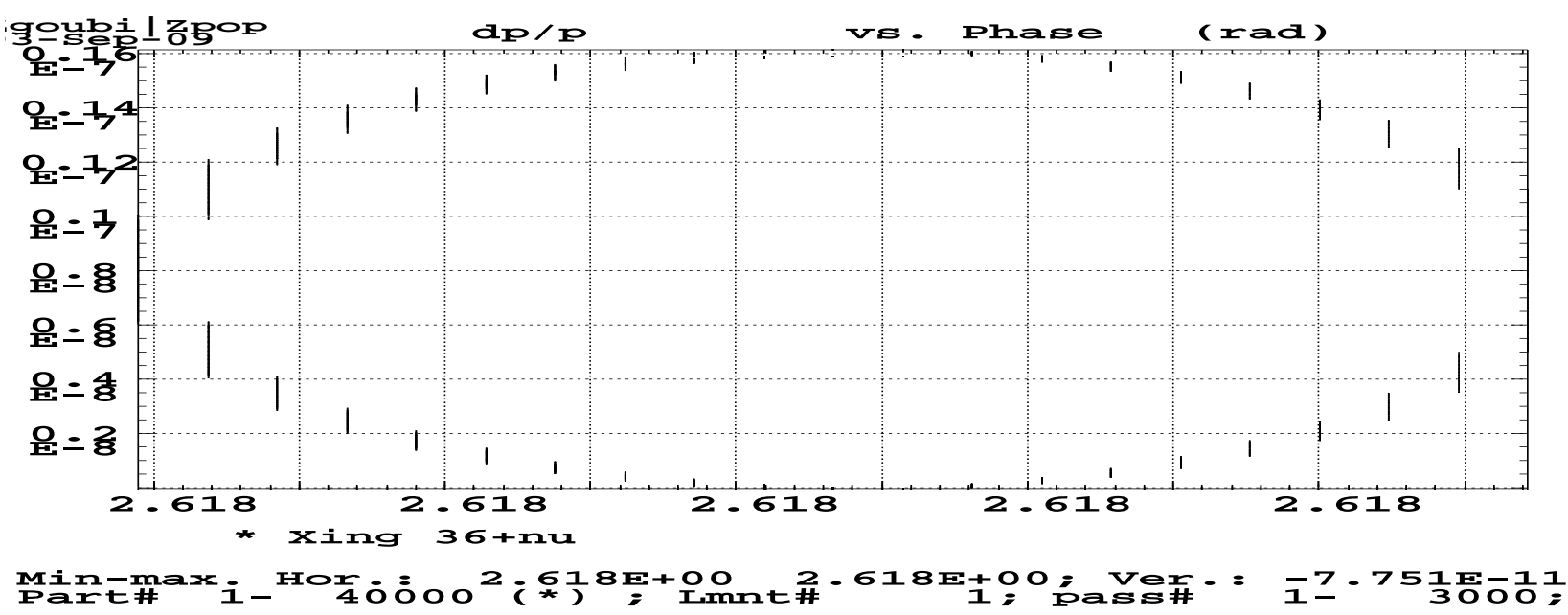

Figure 94: dp-phase. 


\subsection{Imperfection resonances}

\subsection{1 $\gamma G=9(3.77180 \mathrm{GeV})$}

\section{Tracking data}

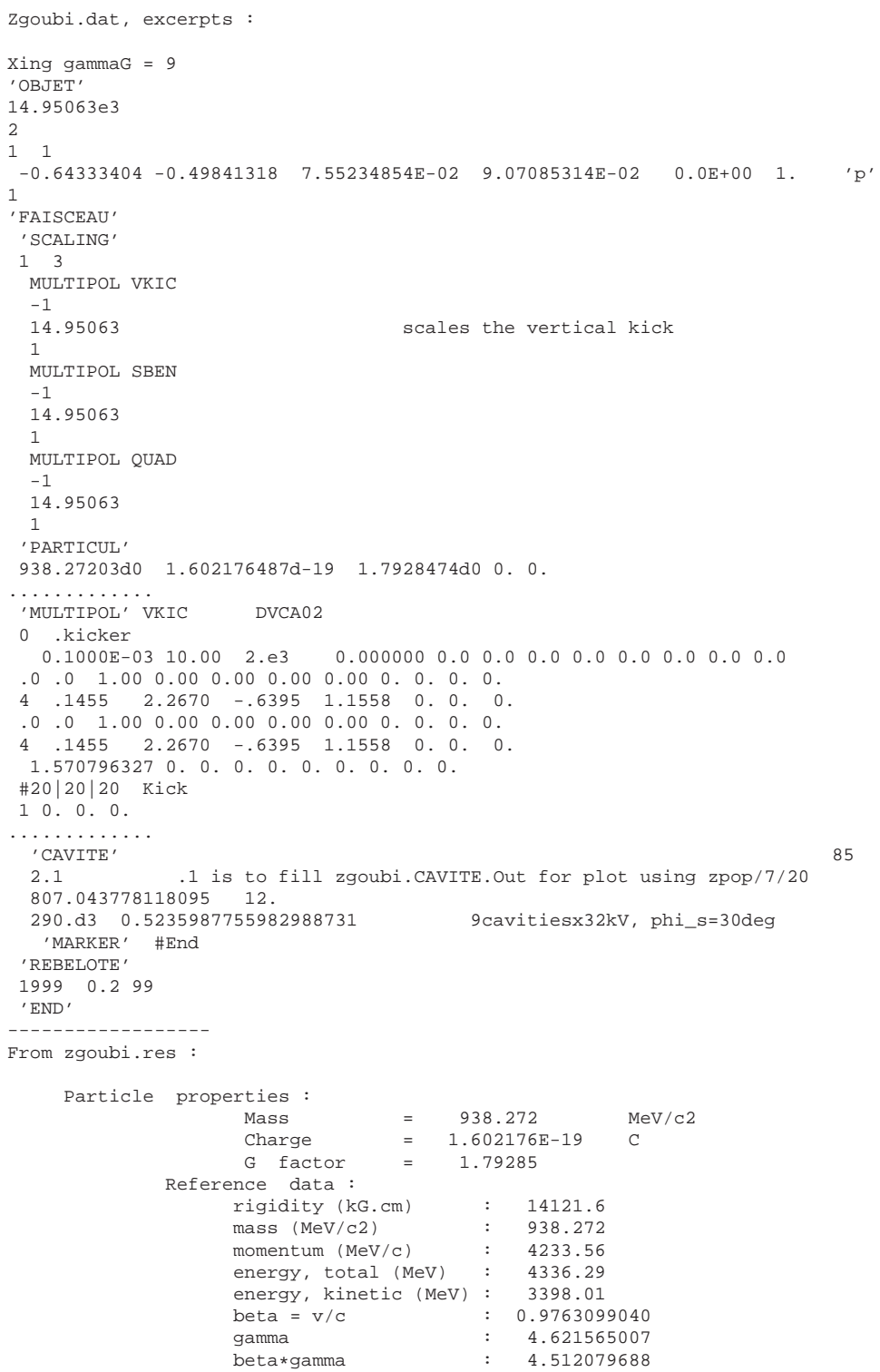

\section{Strengths}

From Figs. 96, 97, Eq. 2, one gets

$\begin{array}{cccccccc}\text { kick } & \hat{z}_{c o} & A^{2} & \left|J_{n}\right|^{2} & A^{2} / \hat{z}_{c o} & \left|J_{n}\right|^{2} / \hat{z}_{c o} & p_{\text {init }} & p_{\text {final }} \\ (\mathrm{mrad}) & (\mathrm{mm}) & & \left(10^{-6}\right) & & & & \\ 0.1 & 0.093 & & & & 4.1295695 & & 0.997457 \\ 1 & 0.93 & & & & 4.1102805 & 1 . & 0.762110 \\ & 2.77 & 1.224737 & 34.381555 & & & 0.992 & -0.412\end{array}$




$$
\gamma G=9-\hat{z}_{c o}=2.77 \mathrm{~mm}
$$

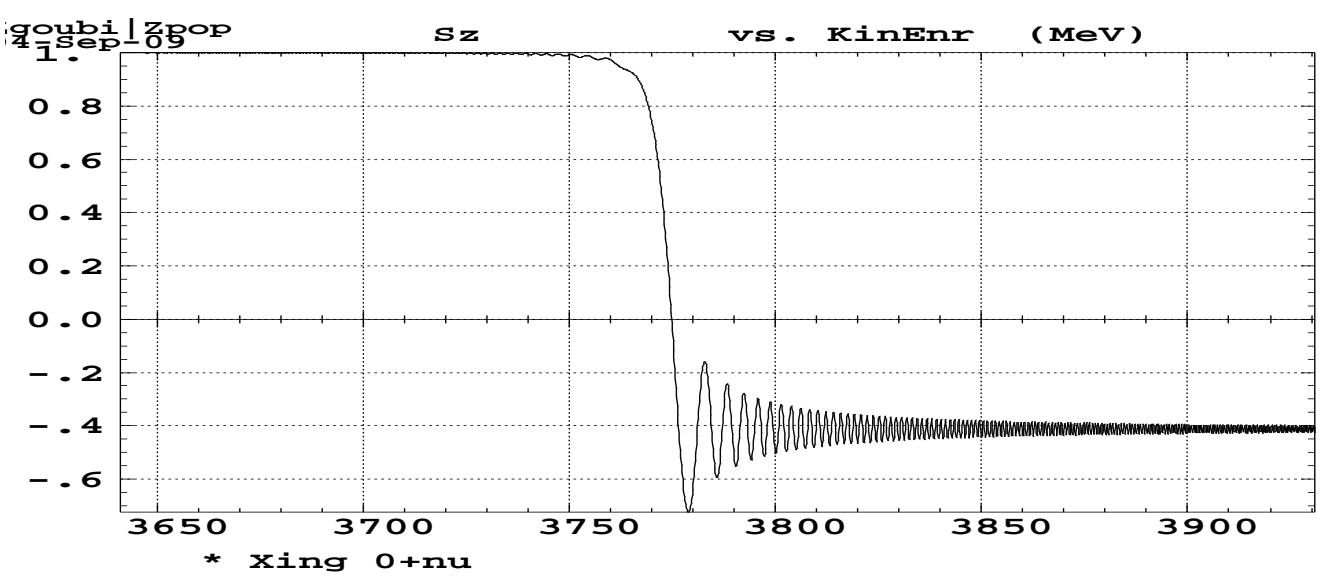

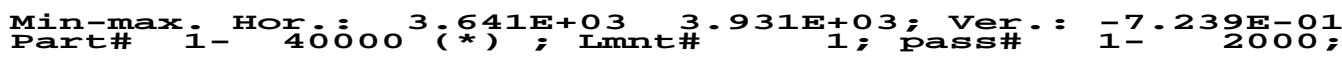

Figure 95: $S_{z}$ versus kinetic energy.

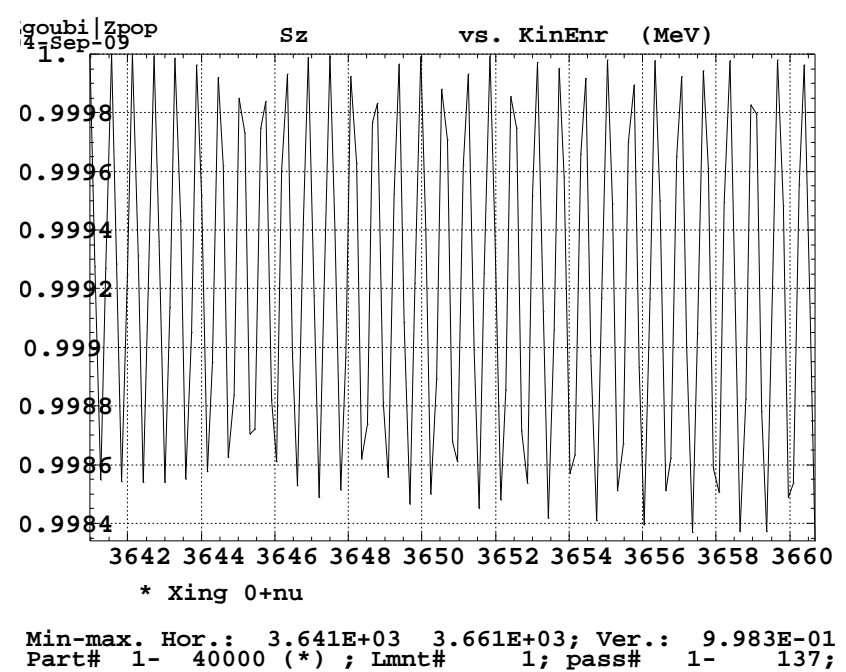

Figure 96: Zoom on initial $S_{z}$.

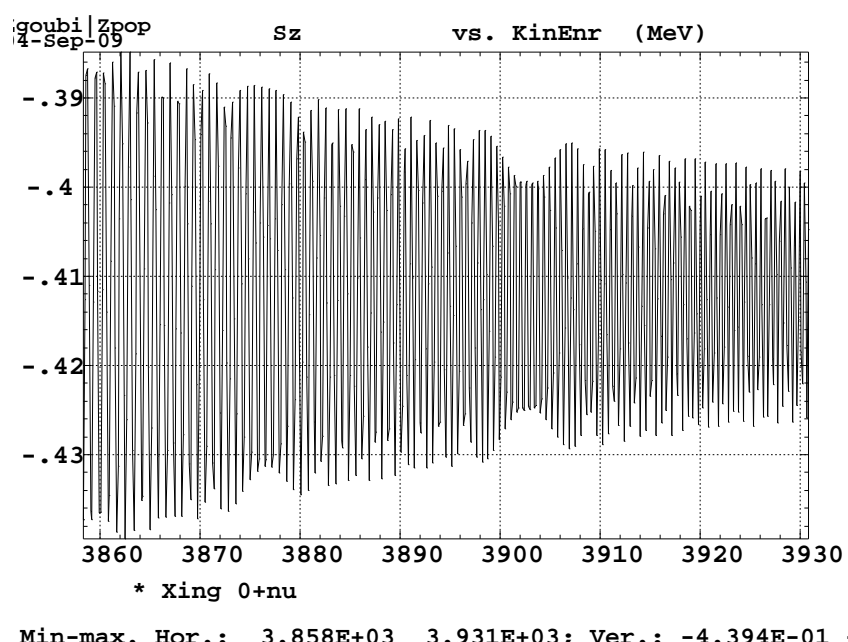

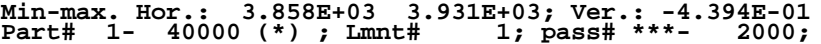

Figure 97: Zoom on final $S_{z}$.

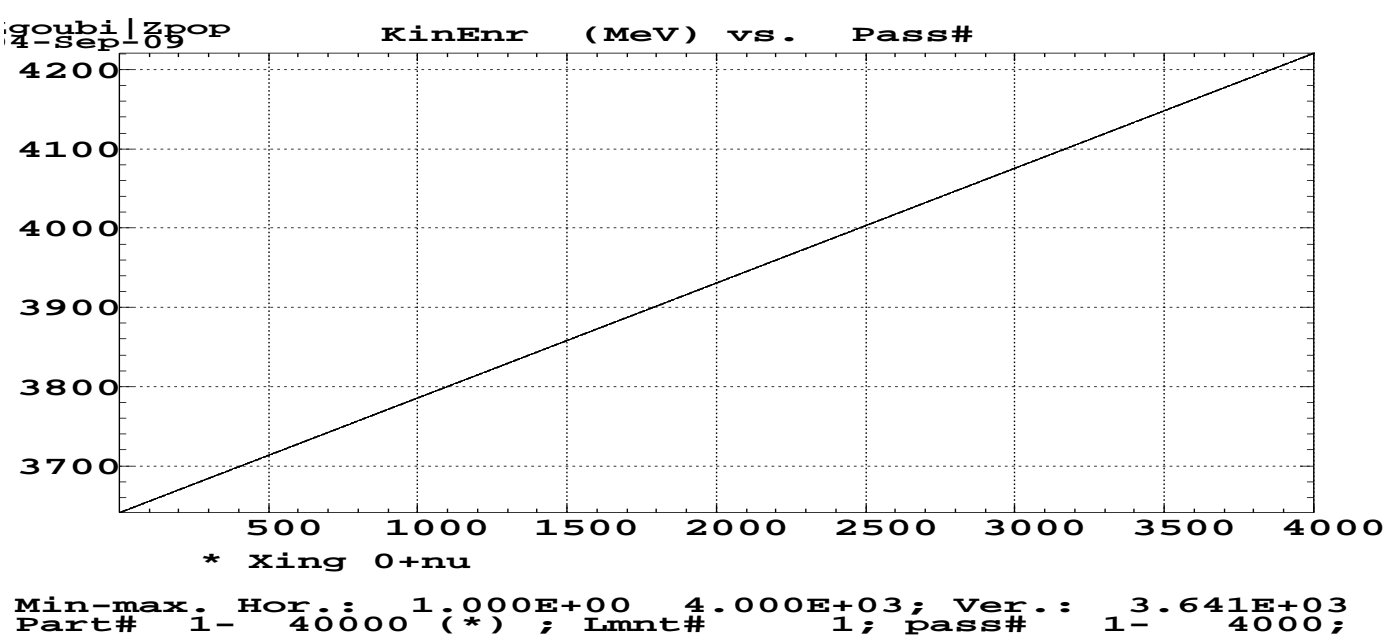

Figure 98: Kinetic E versus turn number. 
$\gamma G=9-\hat{z}_{c o}=2.77 \mathrm{~mm}$
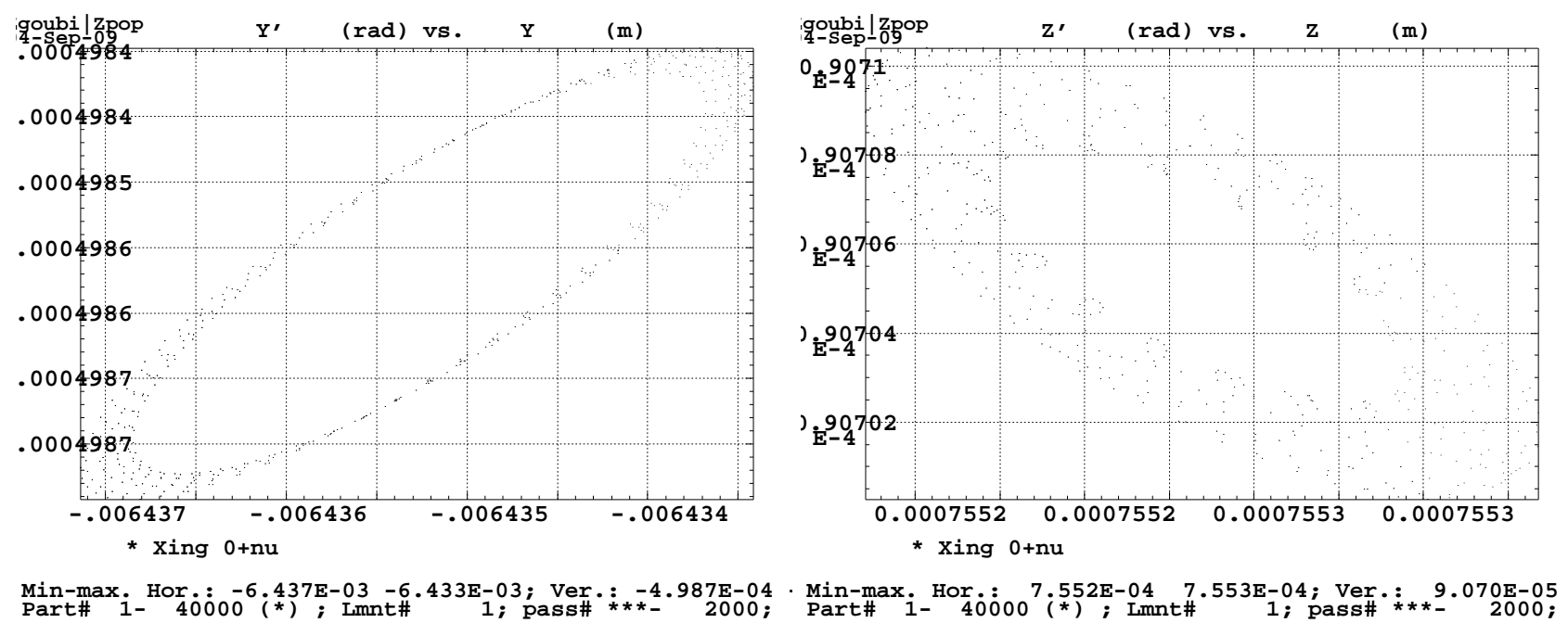

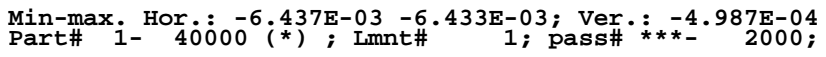

Figure 99: Left : x-x', right : z-z'.

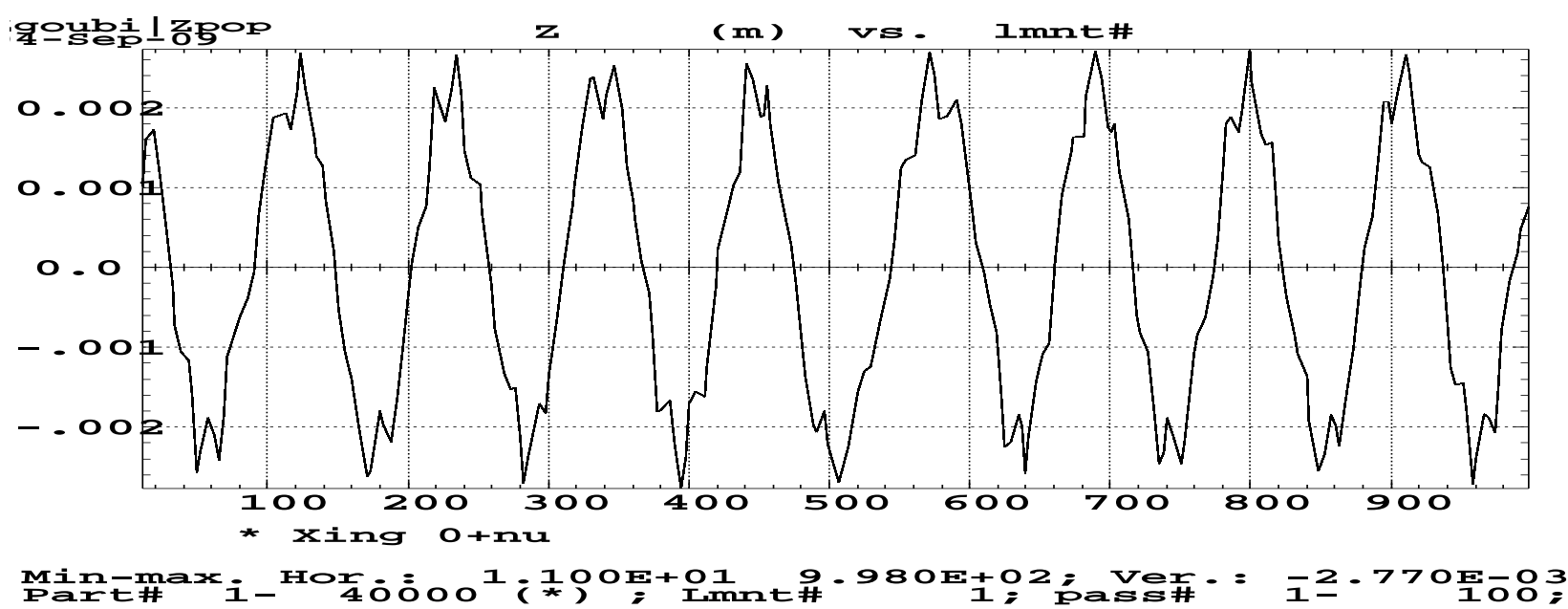

Figure 100: Vertical closed orbit along the ring circumference, versus pick-up number (about 990 P-Us over the $807 \mathrm{~m}$ circumference).

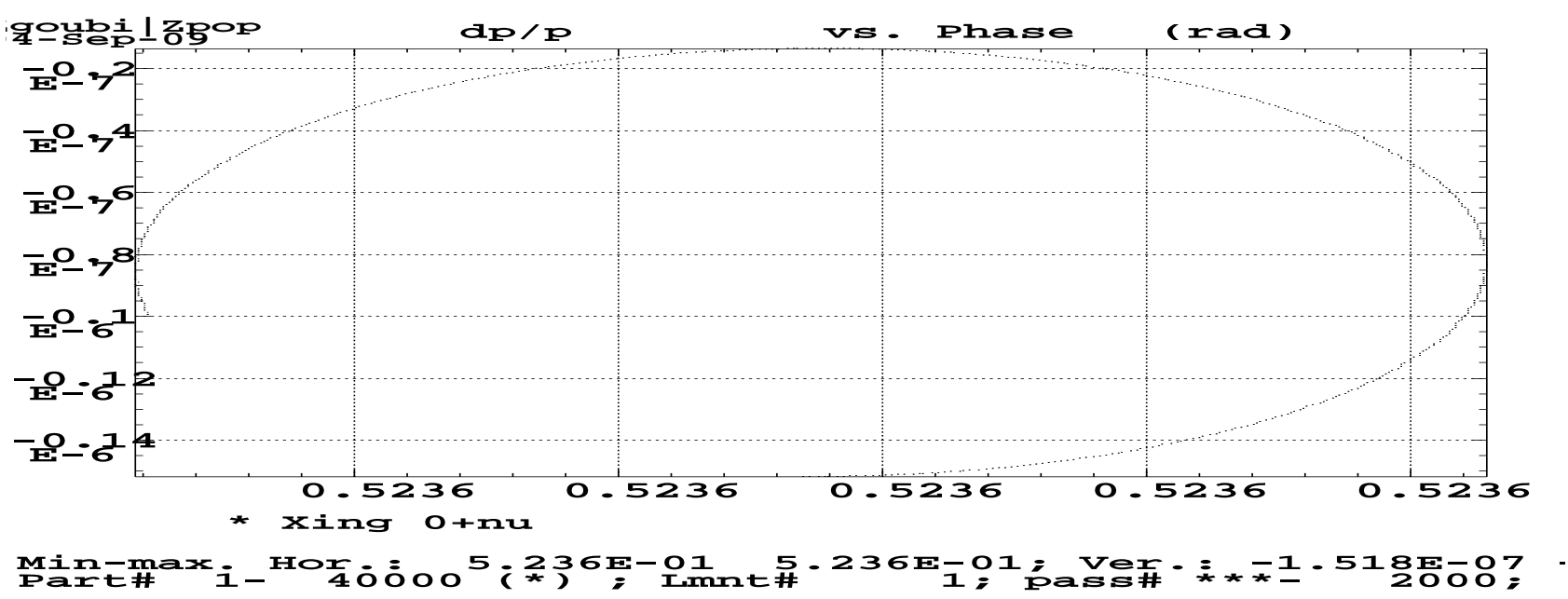

Figure 101: dp-phase. 


\subsection{2 $\gamma G=12(\mathbf{5 . 3 4 1 8 3 0} \mathbf{G e V})$}

\section{Tracking data}

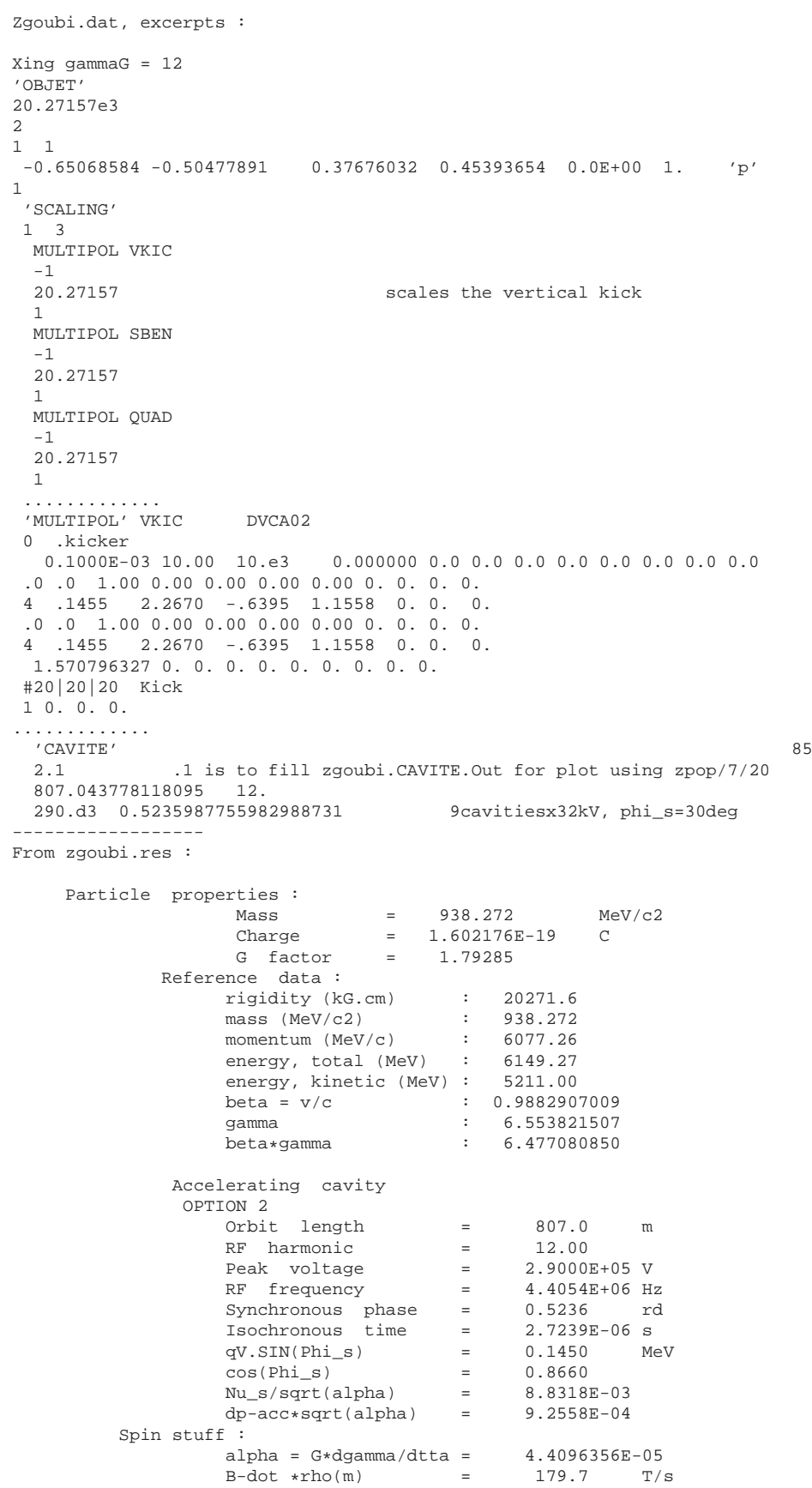

\section{Strength}

From Figs. 103, 104 one gets

$p_{\text {init }} \approx 0.9999, \quad p_{\text {final }} \approx 5.674 E-2$

Eq. 2 yields

$$
\begin{aligned}
& A^{2}=0.6379532 \\
& \left|J_{n}\right|^{2}=9.9891440 E-06
\end{aligned}
$$




$$
\gamma G=12(5.341830 \mathrm{GeV})
$$

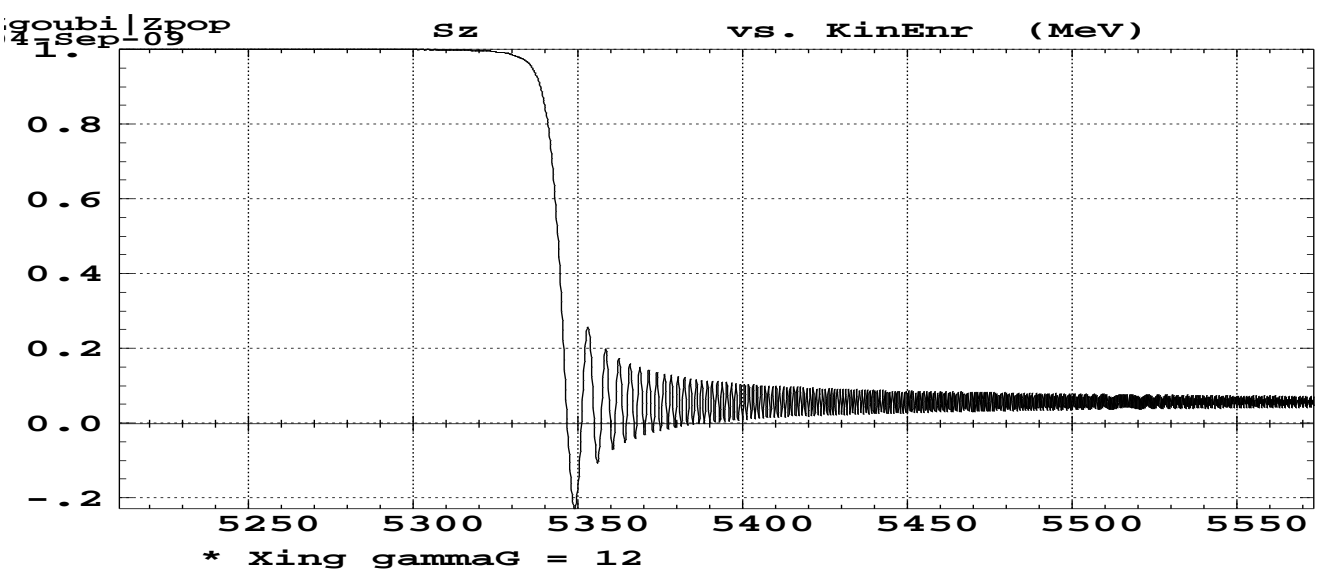

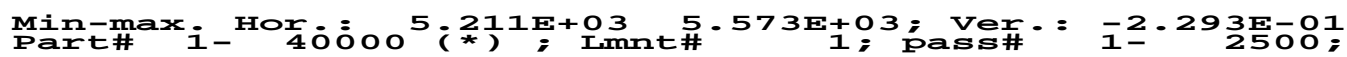

Figure 102: $S_{z}$ versus kinetic energy.

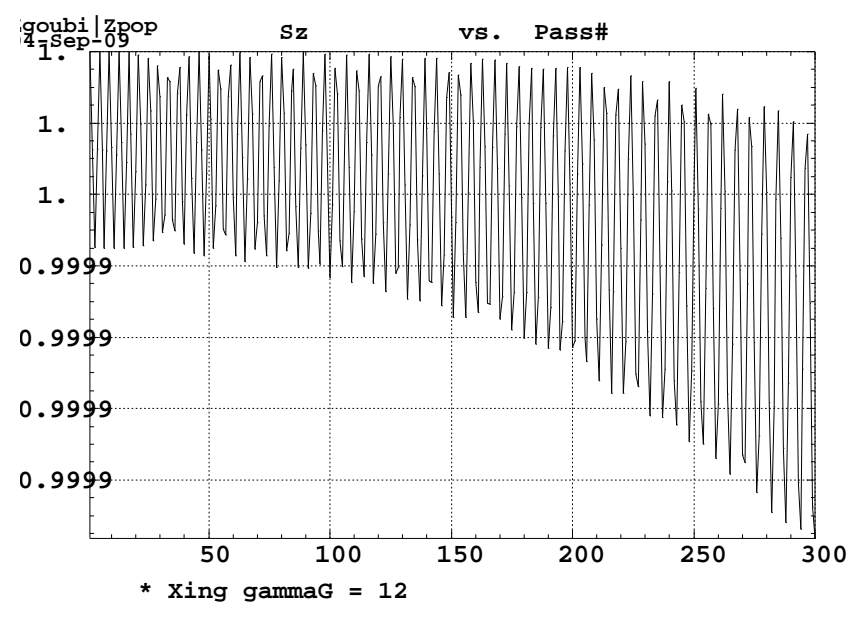

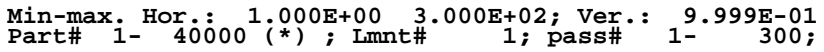

Figure 103: Zoom on initial $S_{z}$.

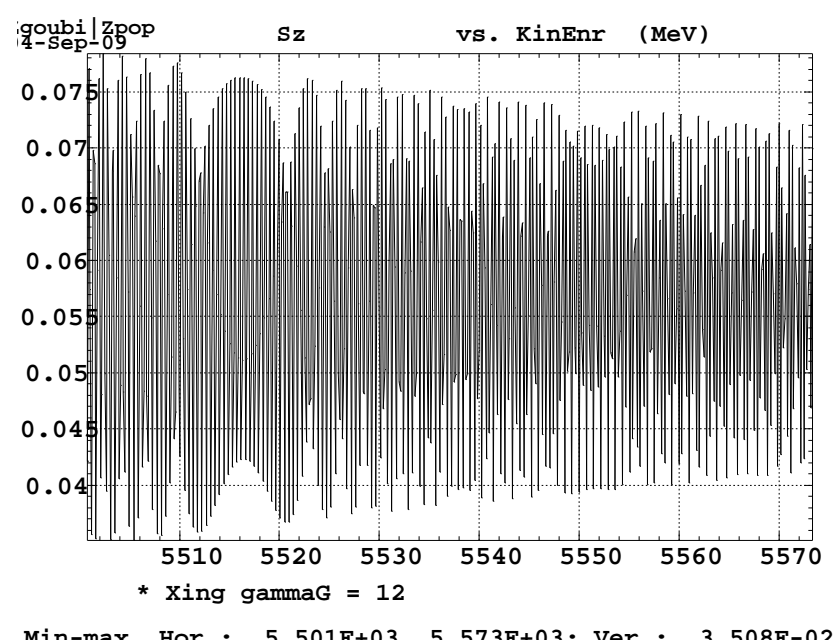

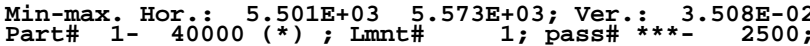

Figure 104: Zoom on final $S_{z}$.

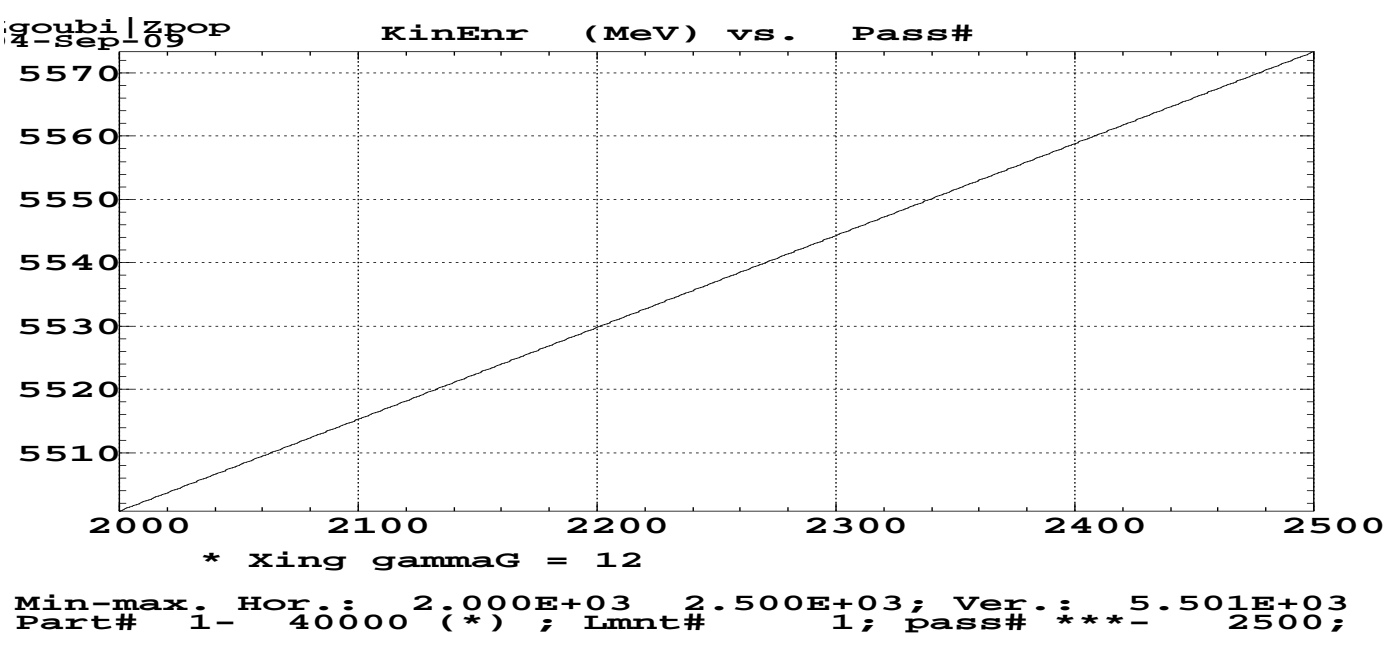

Figure 105: Kinetic E versus turn number. 


$$
\gamma G=12(5.341830 \mathrm{GeV})
$$
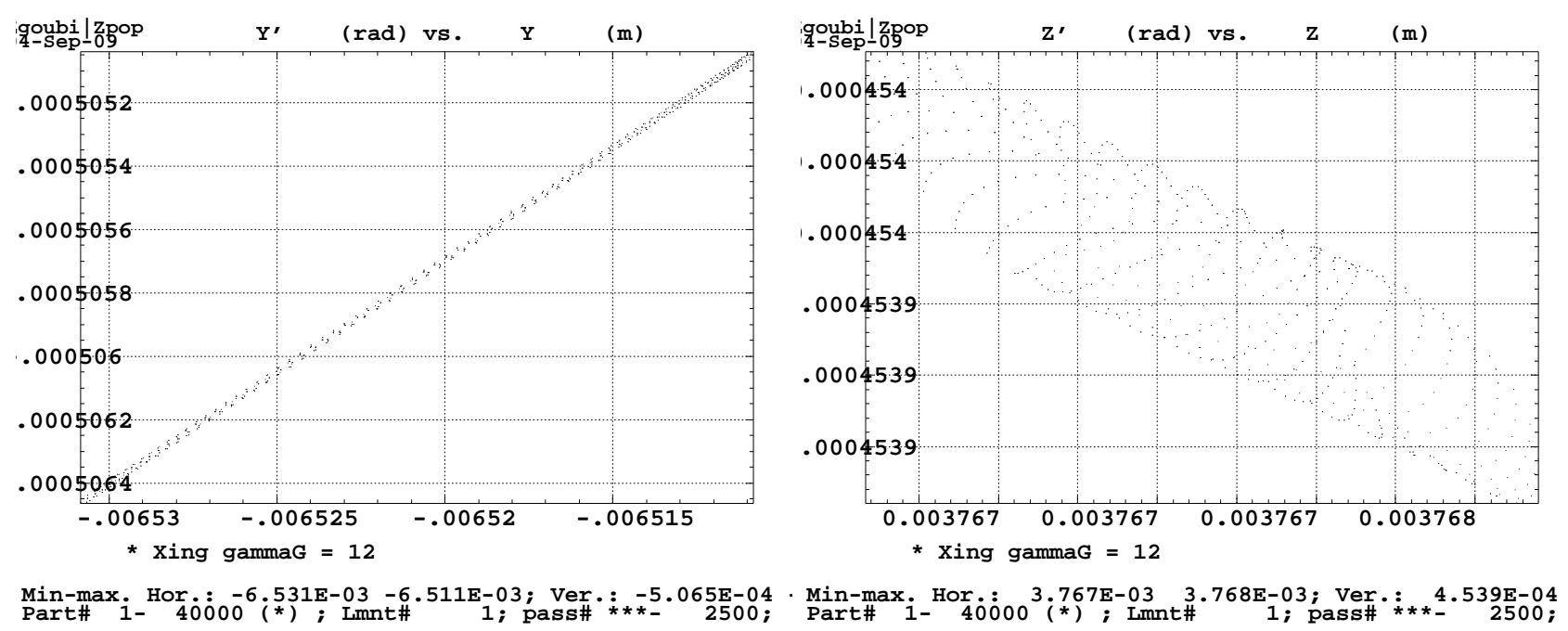

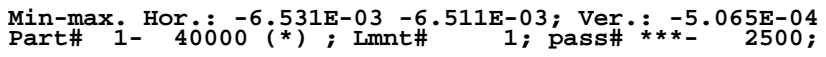

Figure 106: Left : x-x’, right : z-z’.

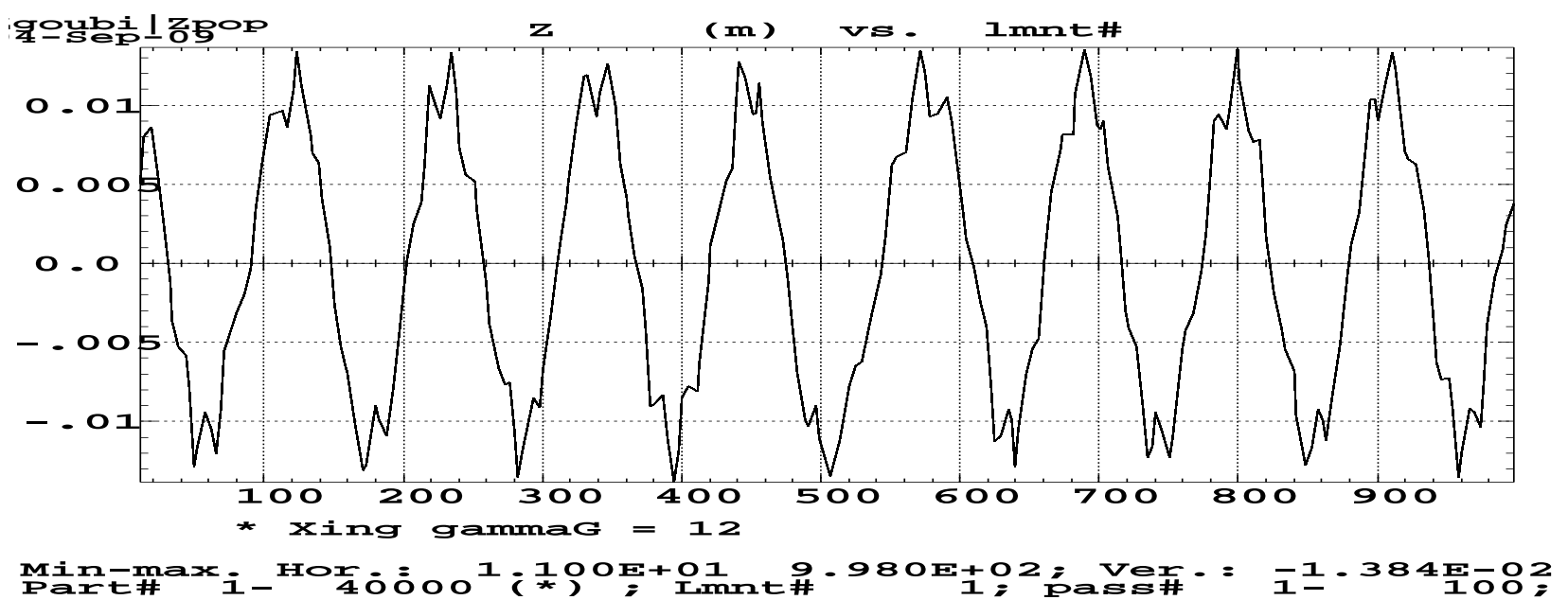

Figure 107: $\mathrm{z}$ closed orbit along the ring circumference versus pick-up number (about 990 P-Us over the $807 \mathrm{~m}$ circumference).

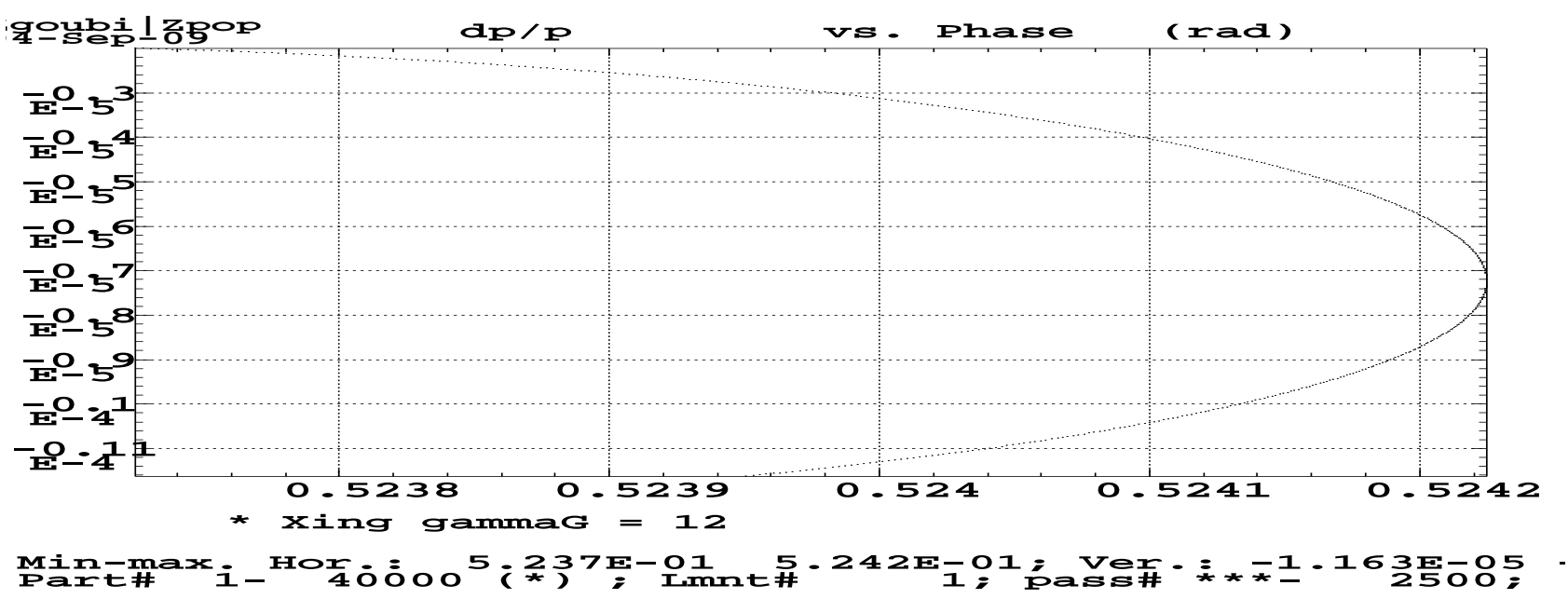

Figure 108: dp-phase. 


\subsection{3 $\gamma G=13(\mathbf{5 . 8 6 5 1 7 1} \mathbf{~ G e V})$}

\section{Tracking data}

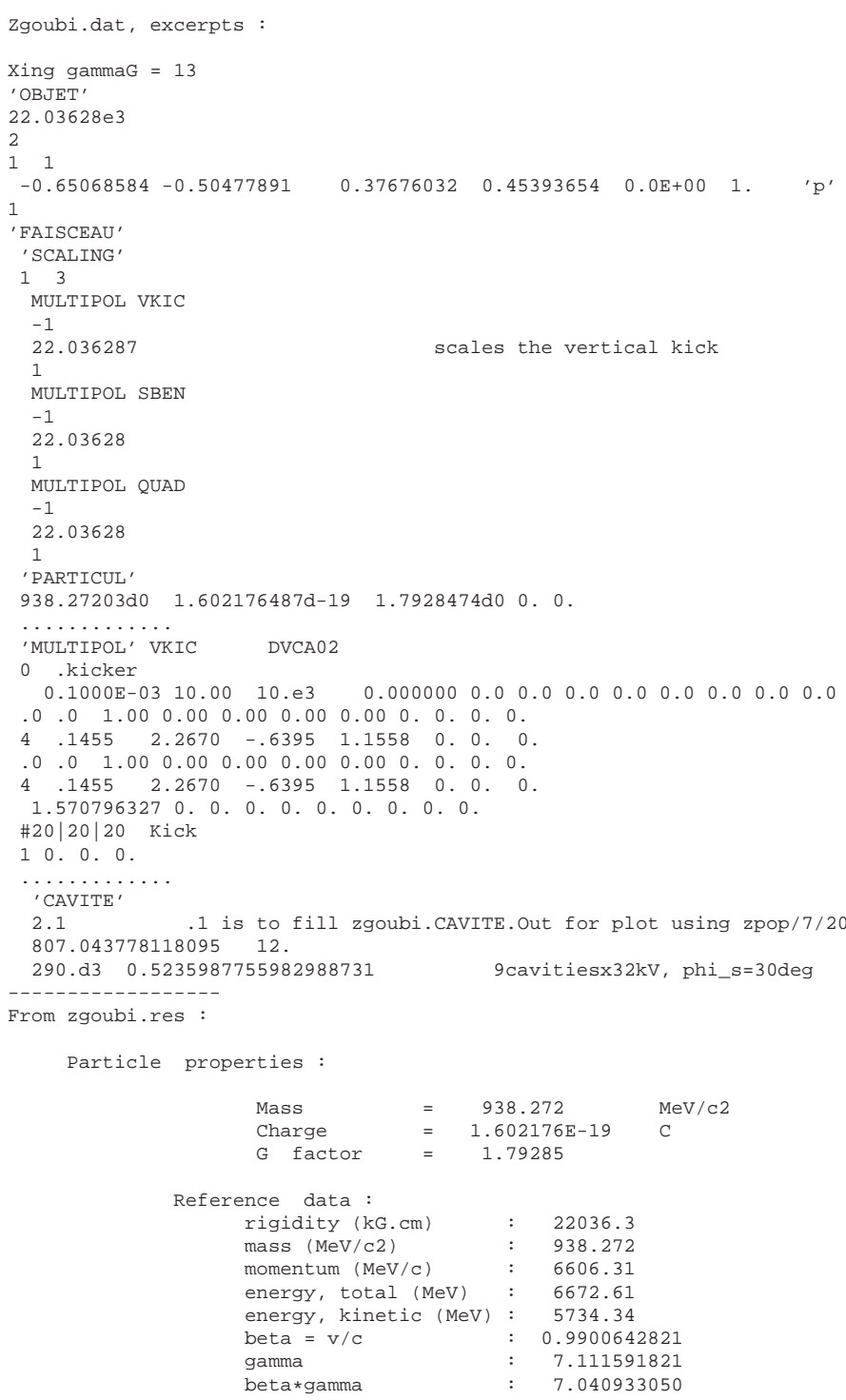

\section{Strength}

From Figs. 110, 111 one gets

$p_{\text {init }} \approx 1 ., \quad p_{\text {final }} \approx 0.1574500$

Eq. 2 yields

$$
\begin{aligned}
& A^{2}=0.5469143 \\
& \left|J_{n}\right|^{2}=1.5353313 E-05
\end{aligned}
$$




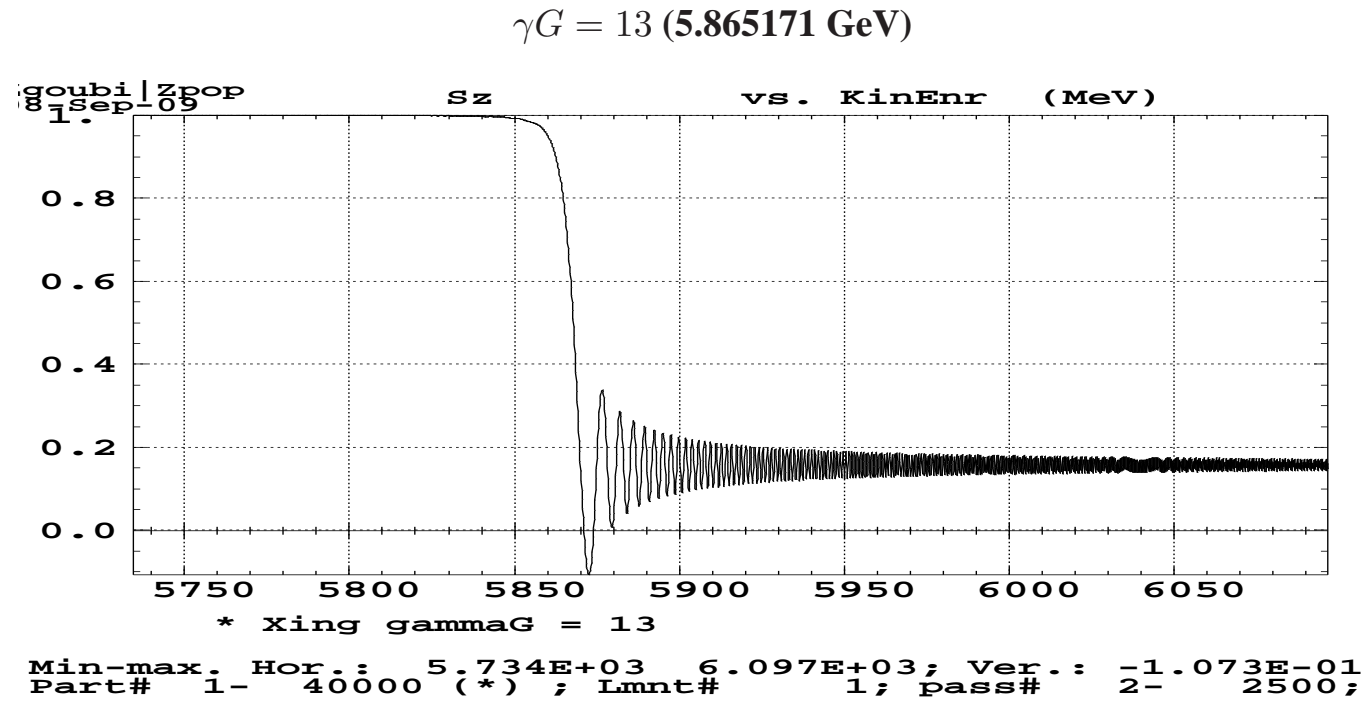

Figure 109: $S_{z}$ versus kinetic energy.

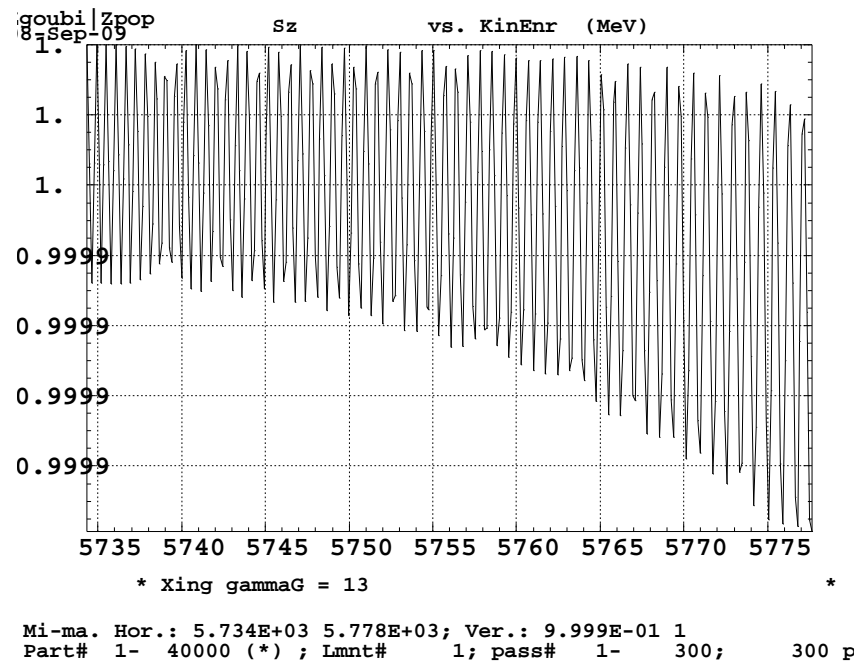

Figure 110: Zoom on initial $S_{z}$.

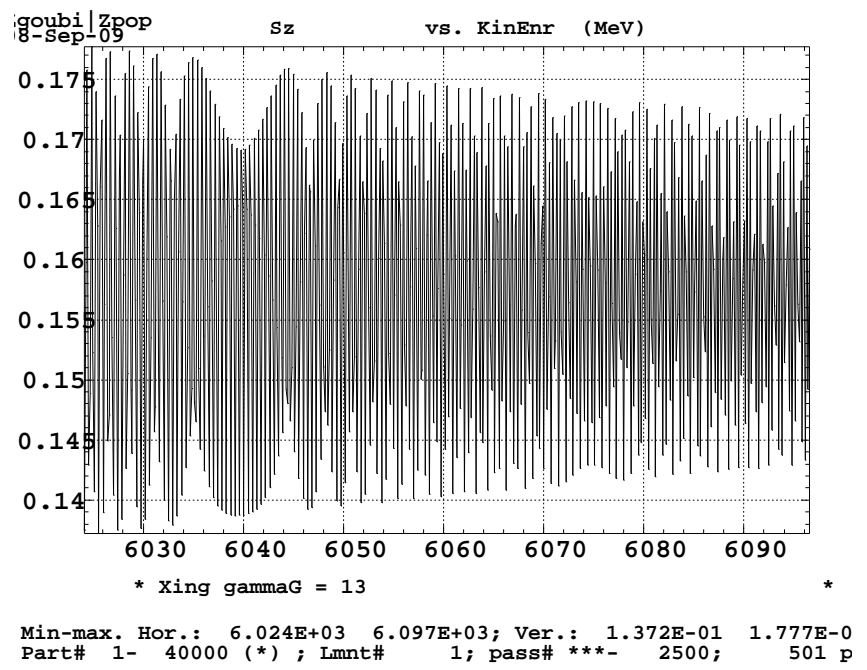

Figure 111: Zoom on final $S_{z}$.

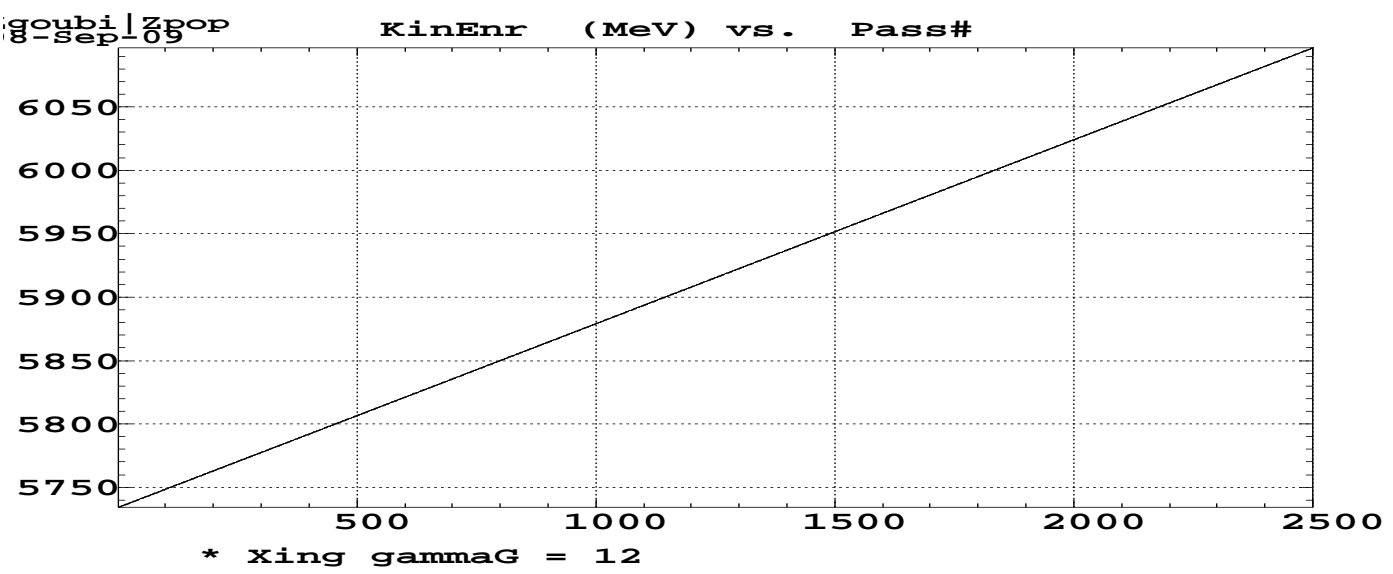

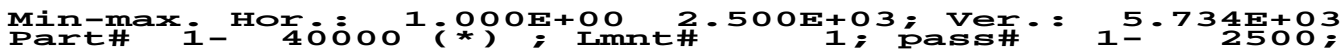

Figure 112: Kinetic E versus turn number. 


$$
\gamma G=13(\mathbf{5 . 8 6 5 1 7 1} \mathbf{G e V})
$$
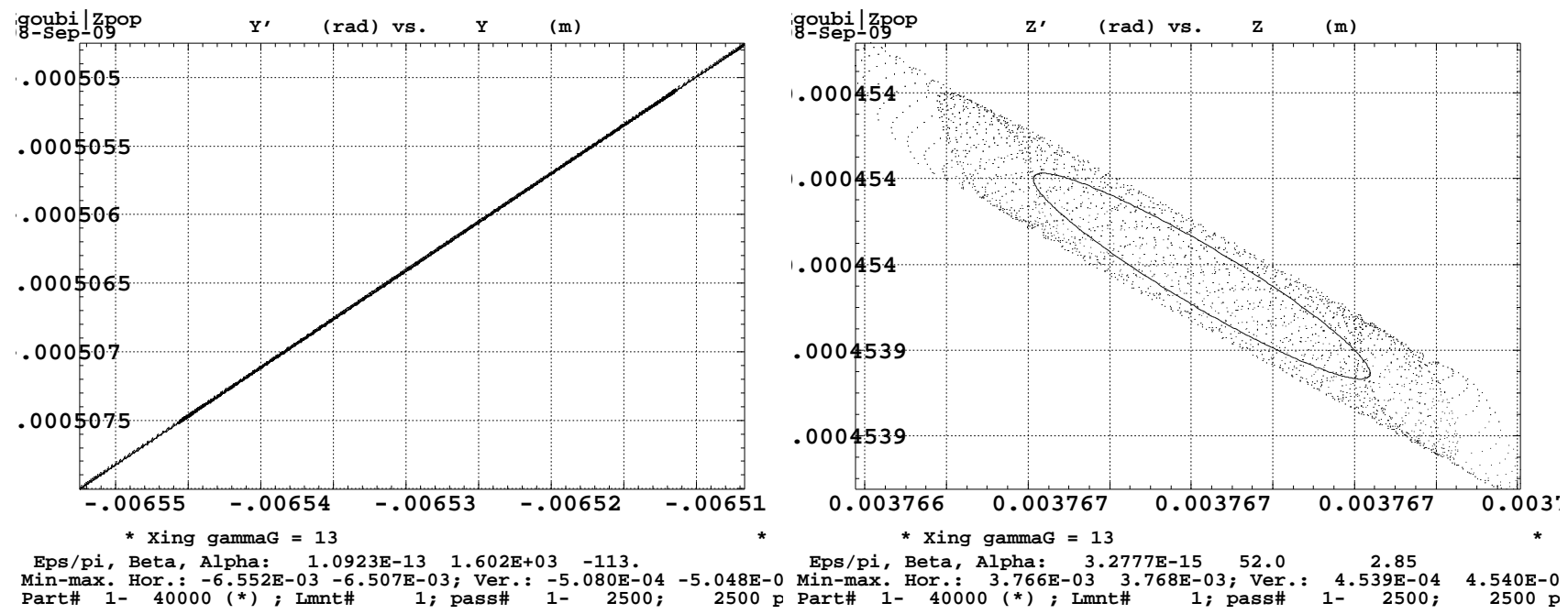

Figure 113: Left : x-x’, right : z-z'.

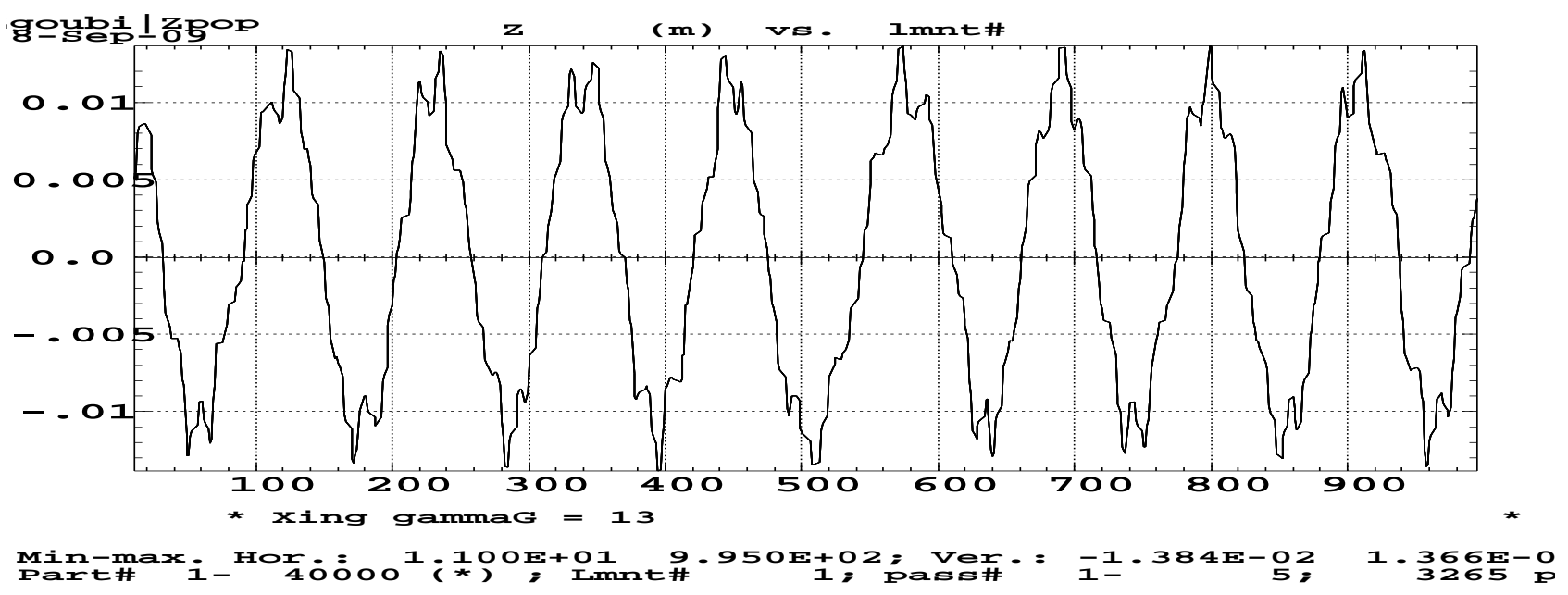

Figure 114: $\mathrm{z}$ closed orbit along the ring circumference (horizontal axis is pick-up number).

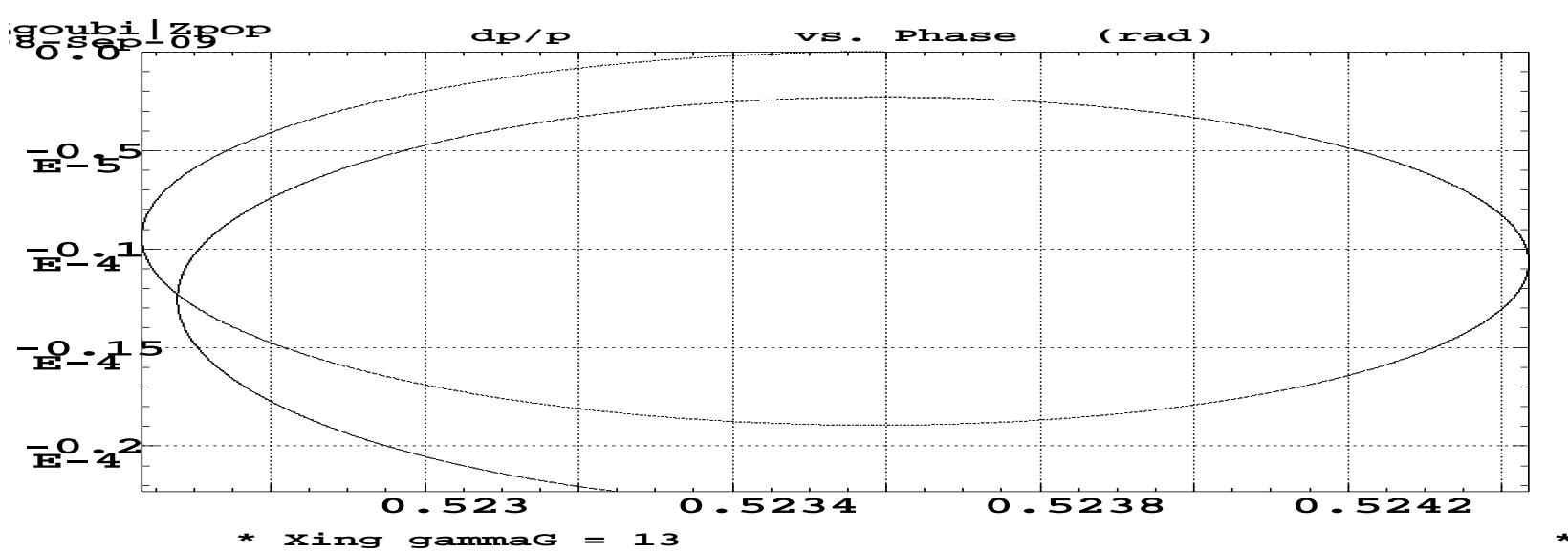

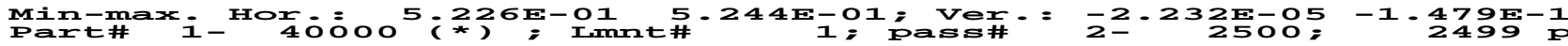

Figure 115: dp-phase. 


\subsection{4 $\gamma G=23(\mathbf{1 1 . 0 9 8 5 9} \mathbf{G e V})$}

\section{Tracking data}

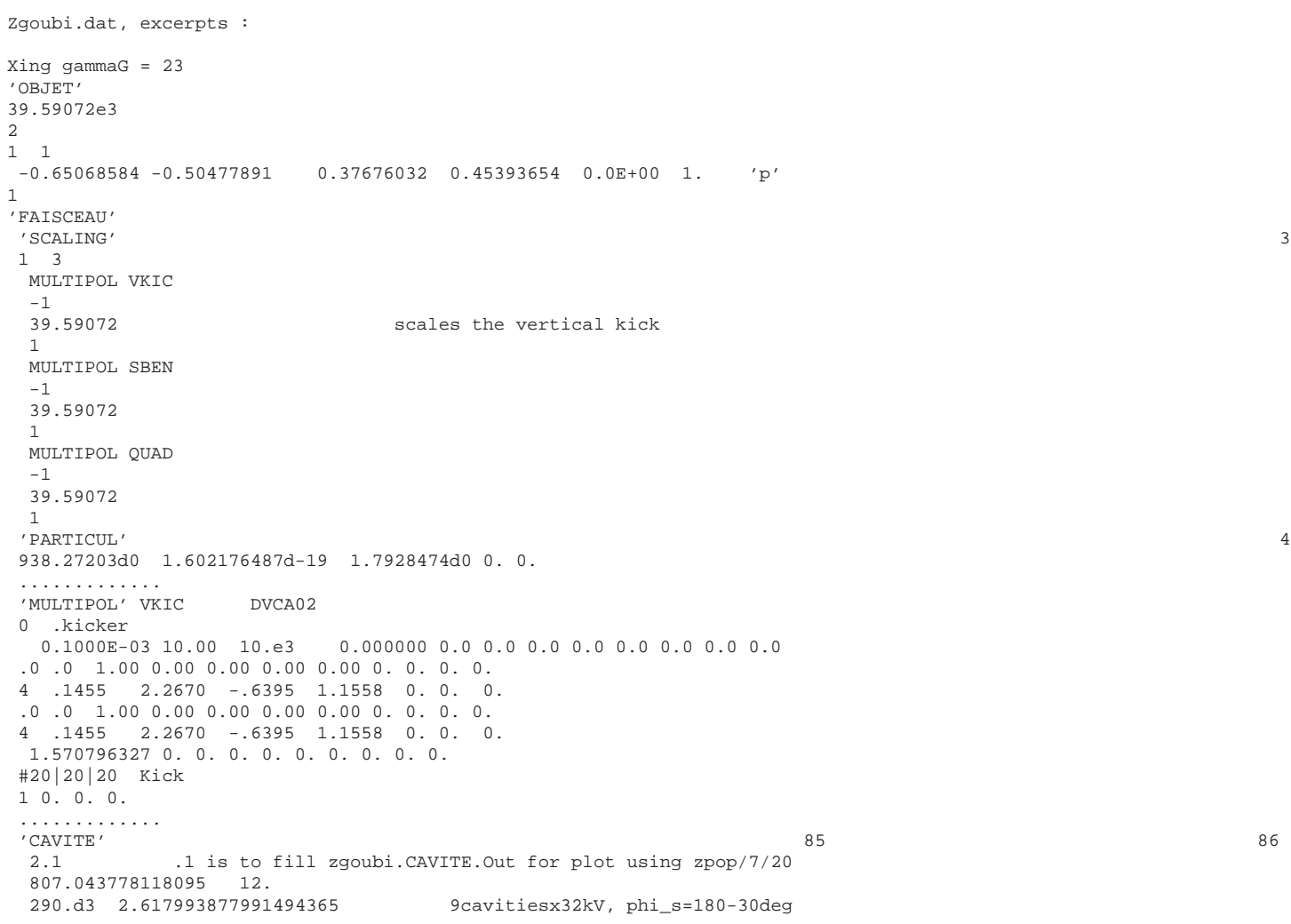

\section{Strength}

From Figs. 117, 118 one gets

$p_{\text {init }} \approx 0.9975, p_{\text {final }} \approx-0.647$

Eq. 2 yields

$A^{2}=1.734326$

$\left|J_{n}\right|^{2}=4.8687059 E-05$ 


$$
\gamma G=23(\mathbf{1 1 . 0 9 8 5 9} \mathbf{G e V})
$$

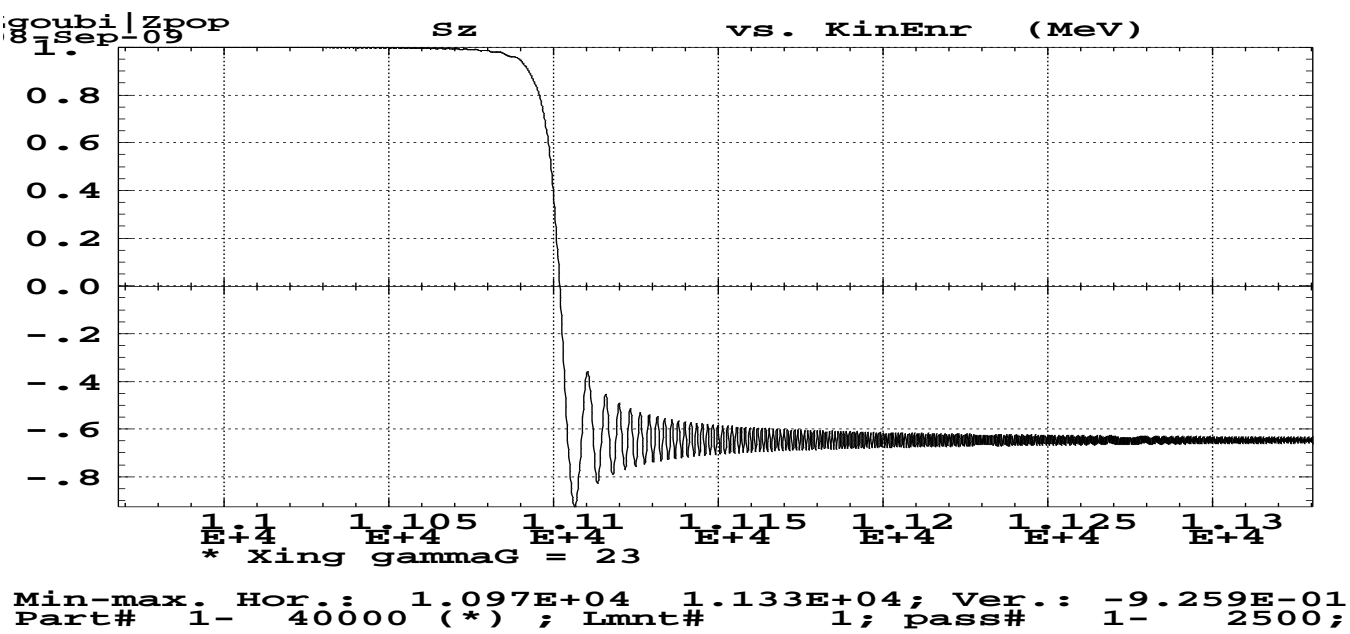

Figure 116: $S_{z}$ versus kinetic energy.

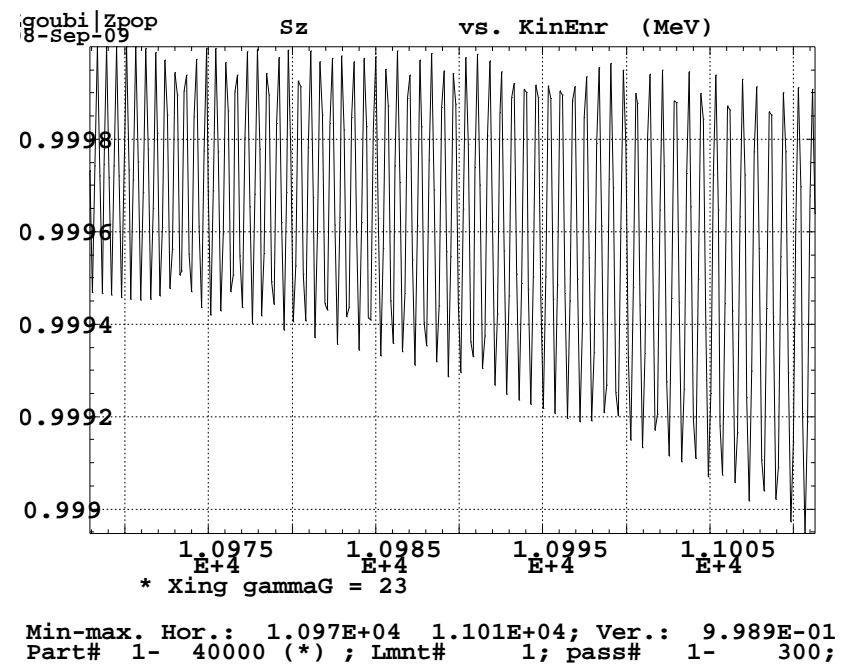

Figure 117: Zoom on initial $S_{z}$.

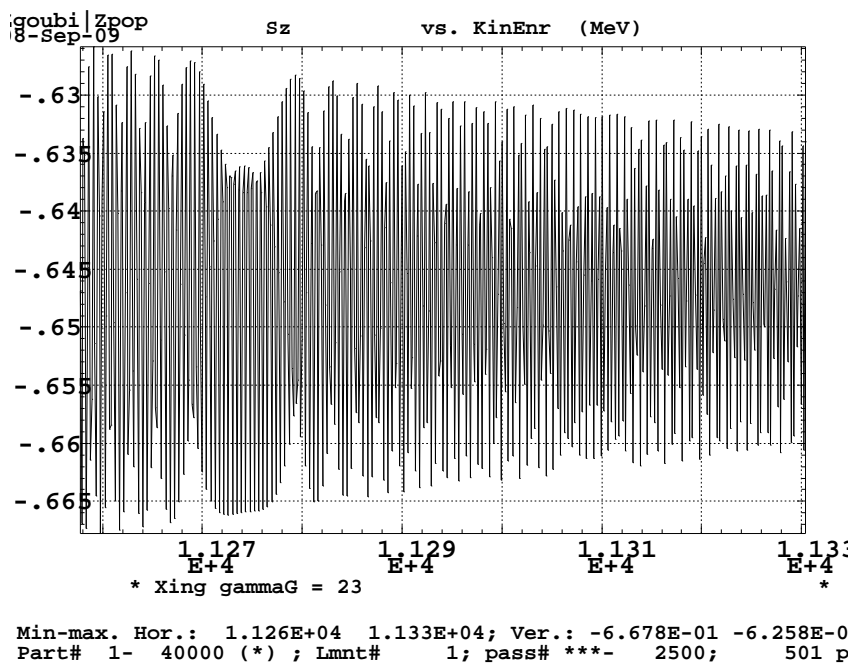

Figure 118: Zoom on final $S_{z}$.

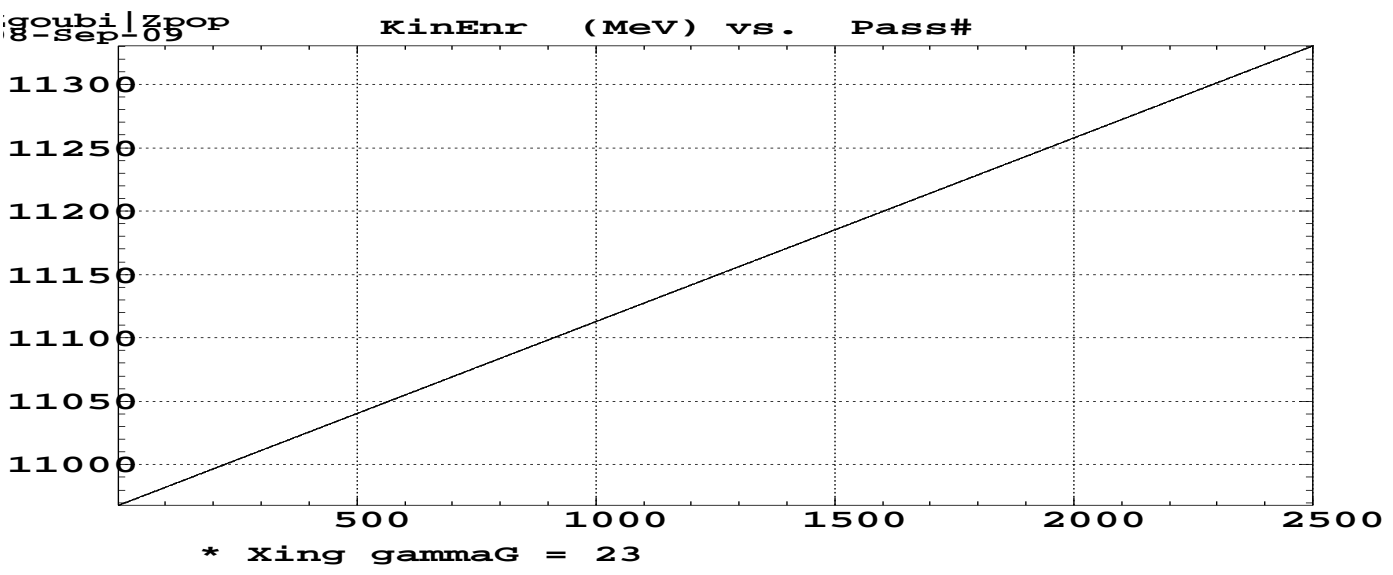

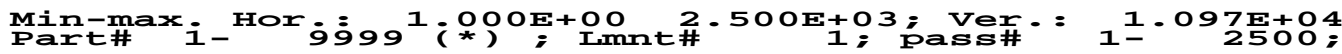

Figure 119: Kinetic E versus turn number. 


$$
\gamma G=23(\mathbf{1 1 . 0 9 8 5 9} \mathbf{G e V})
$$
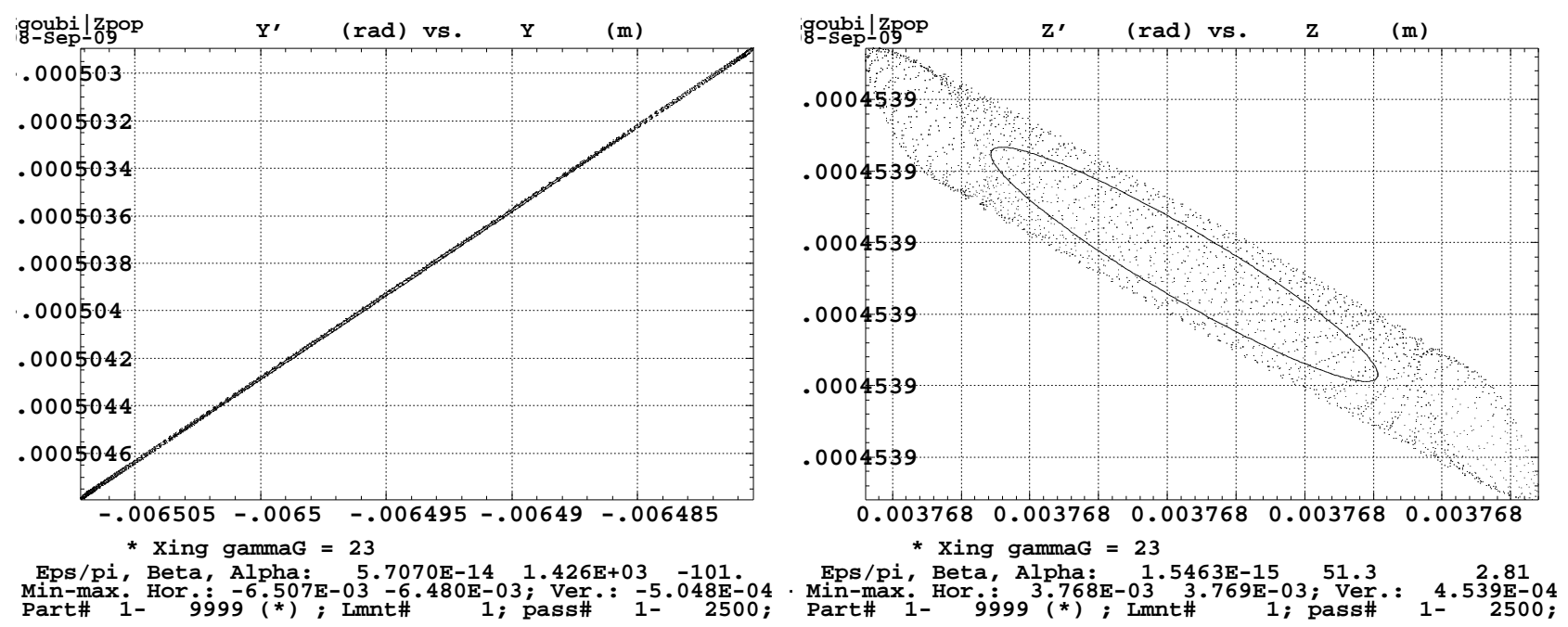

Figure 120: Left : $\mathrm{x}-\mathrm{x}^{\prime}$, right : z-z'.

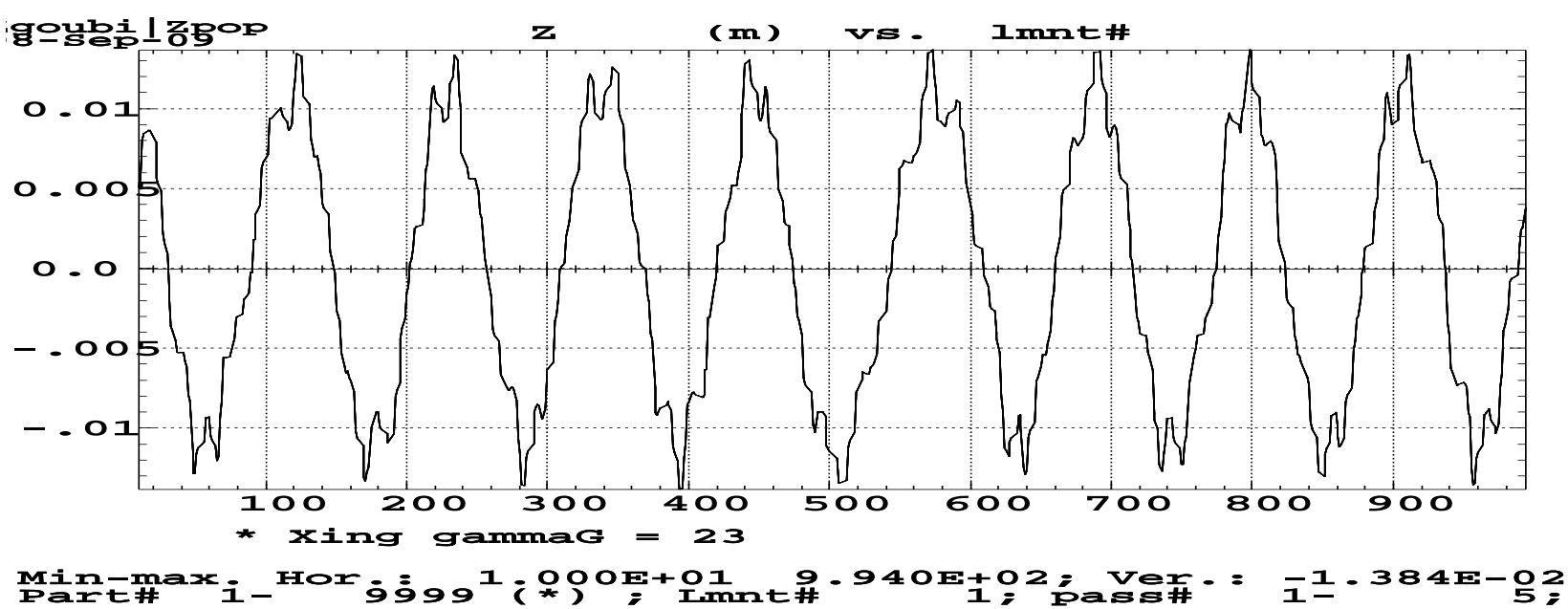

Figure 121: z closed orbit along the ring circumference (horizontal axis is pick-up number).

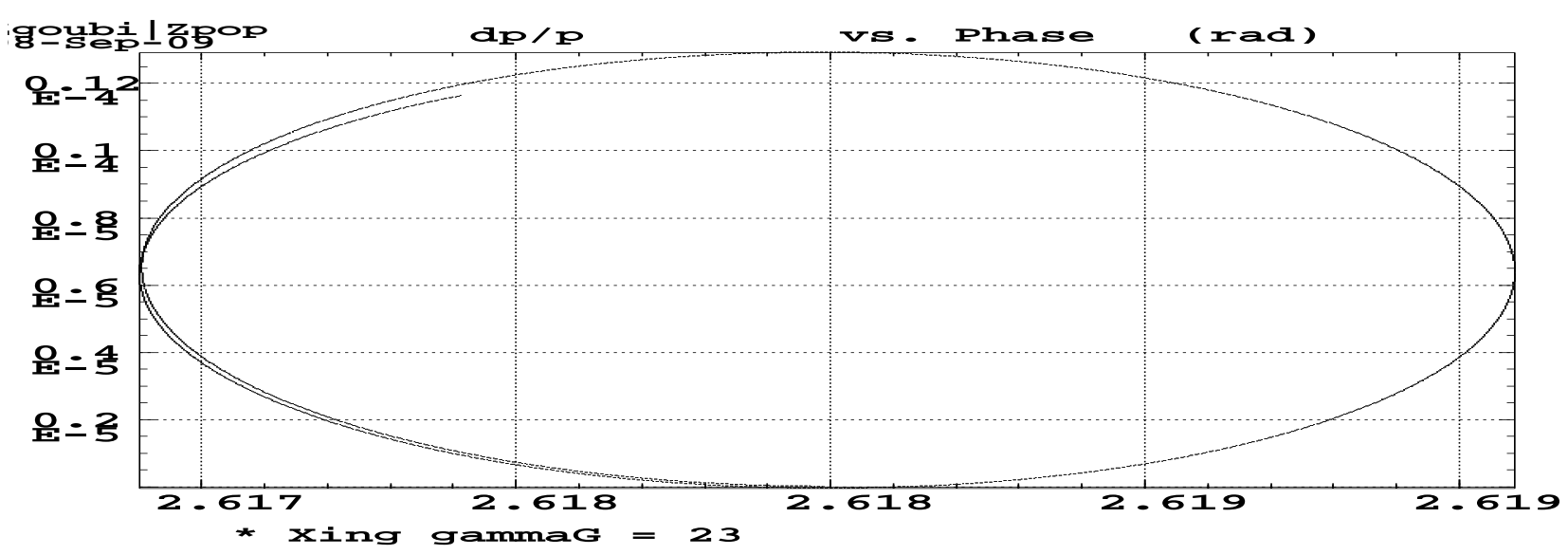

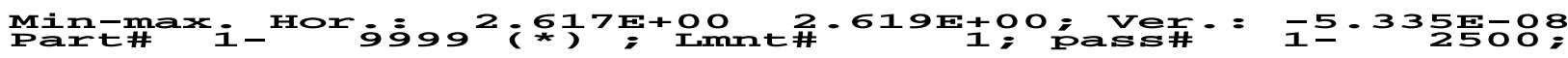

Figure 122: dp-phase. 


\subsection{5 $\gamma G=27(13.19196 \mathrm{GeV})$}

\section{Tracking data}

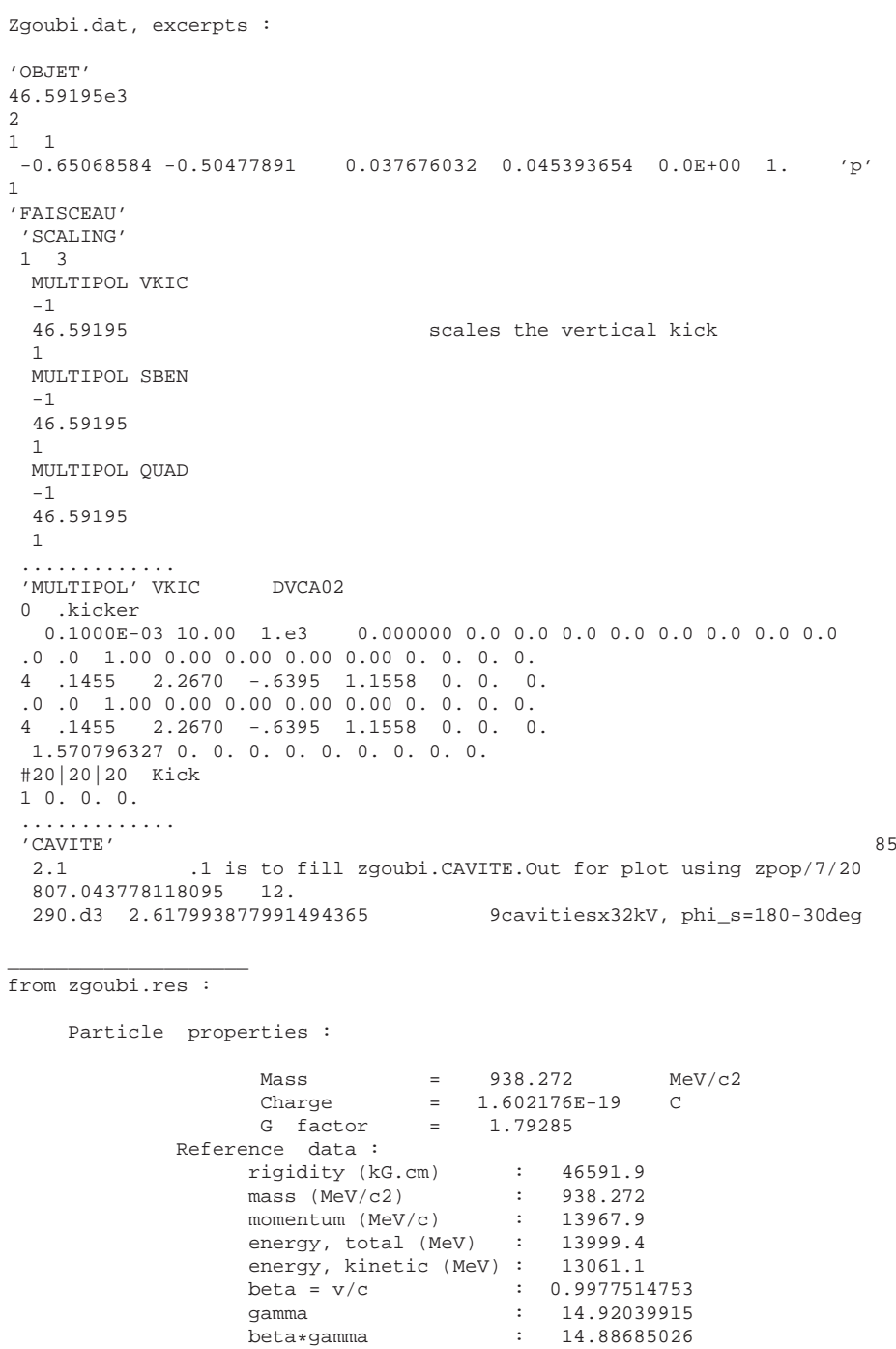

\section{Strength}

From Figs. 124, 125 one gets

$p_{\text {init }} \approx 0.9998, \quad p_{\text {final }} \approx-0.167355$

Eq. 2 yields

$$
\begin{aligned}
& A^{2}=0.8763353 \\
& \left|J_{n}\right|^{2}=2.4601020 E-05
\end{aligned}
$$




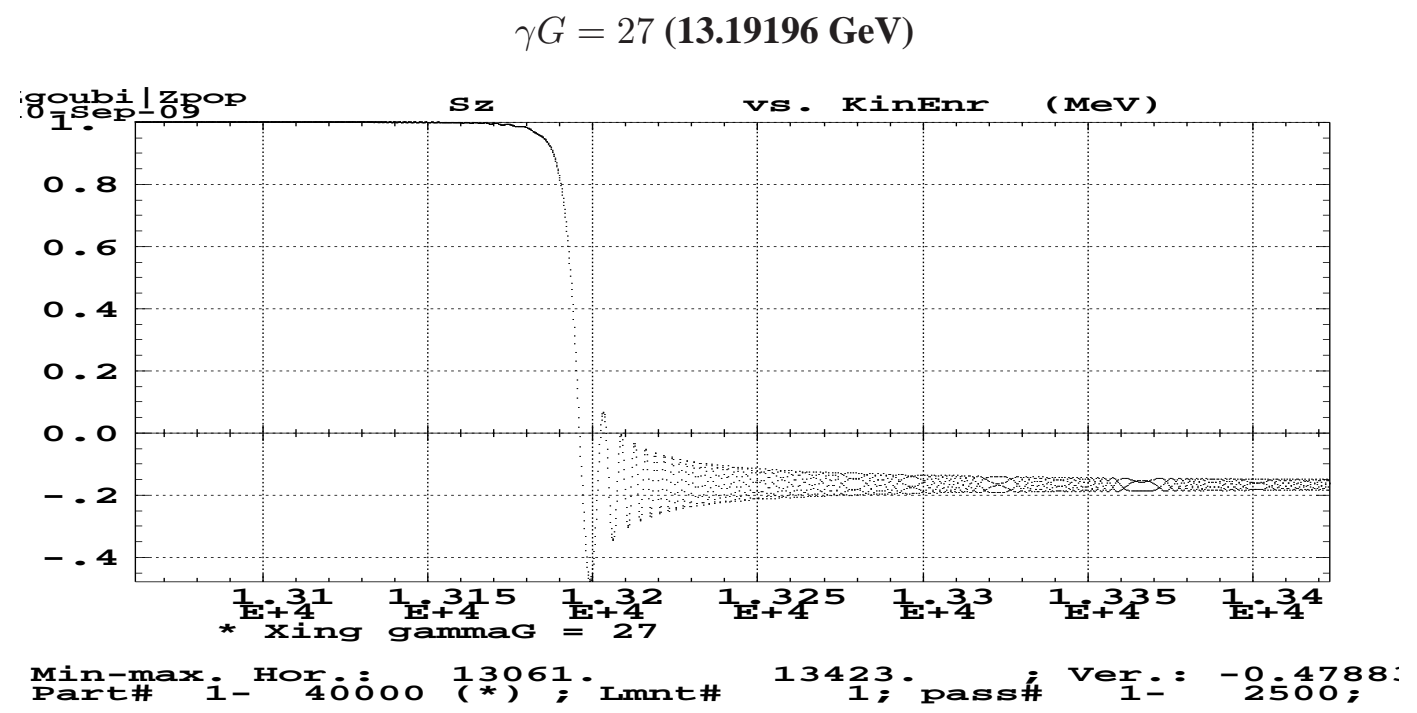

Figure 123: $S_{z}$ versus kinetic energy. Check : 36-Qy next to $\mathrm{gG}=27$ is on the path at $\mathrm{E}=13.31575 \mathrm{GeV}$, yet not visible as expected since $\epsilon_{z} \approx 0$.

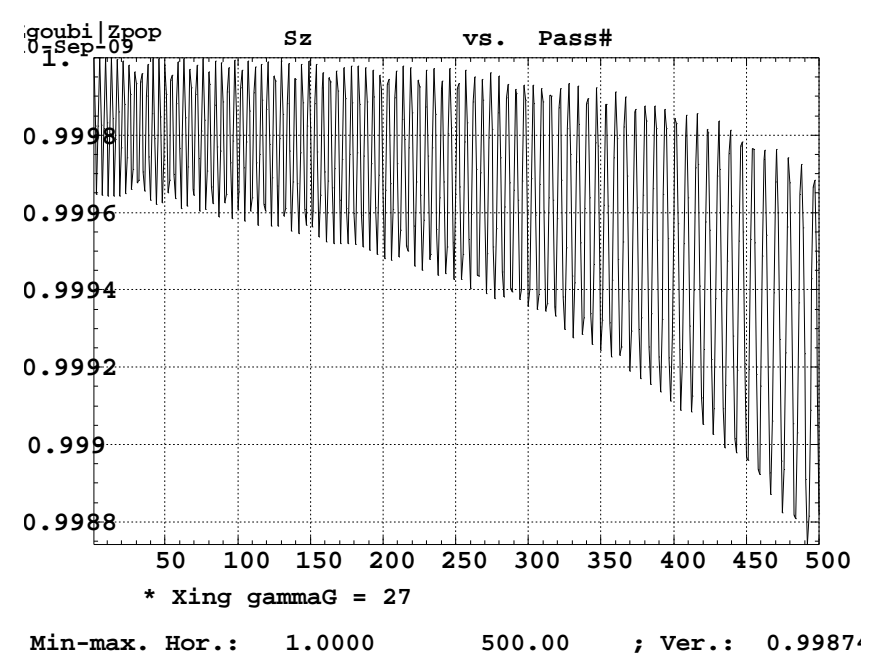

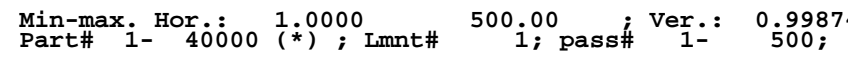

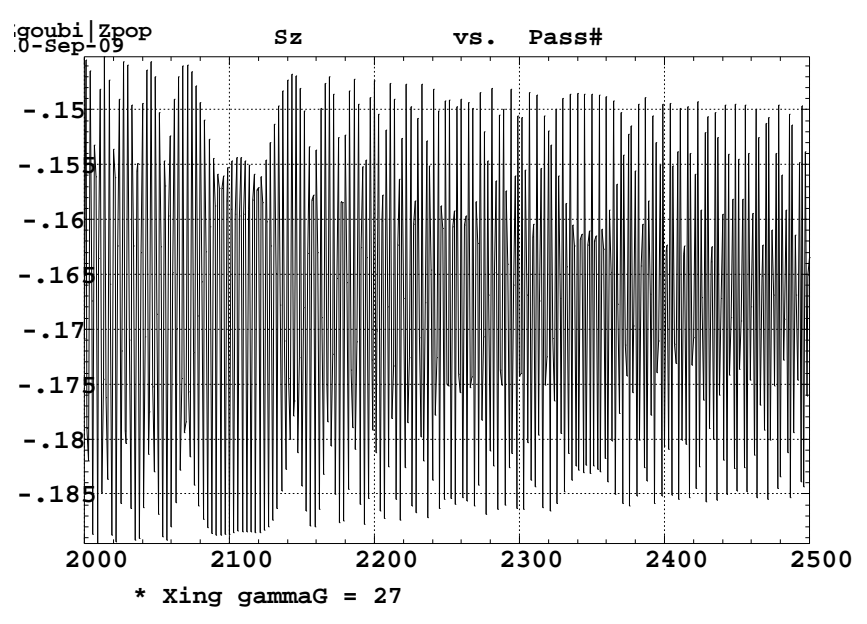

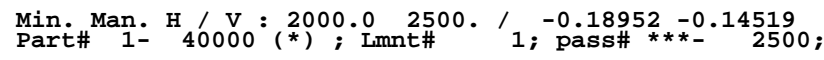

Figure 124: Zoom on initial $S_{z}$.

Figure 125: Zoom on final $S_{z}$.

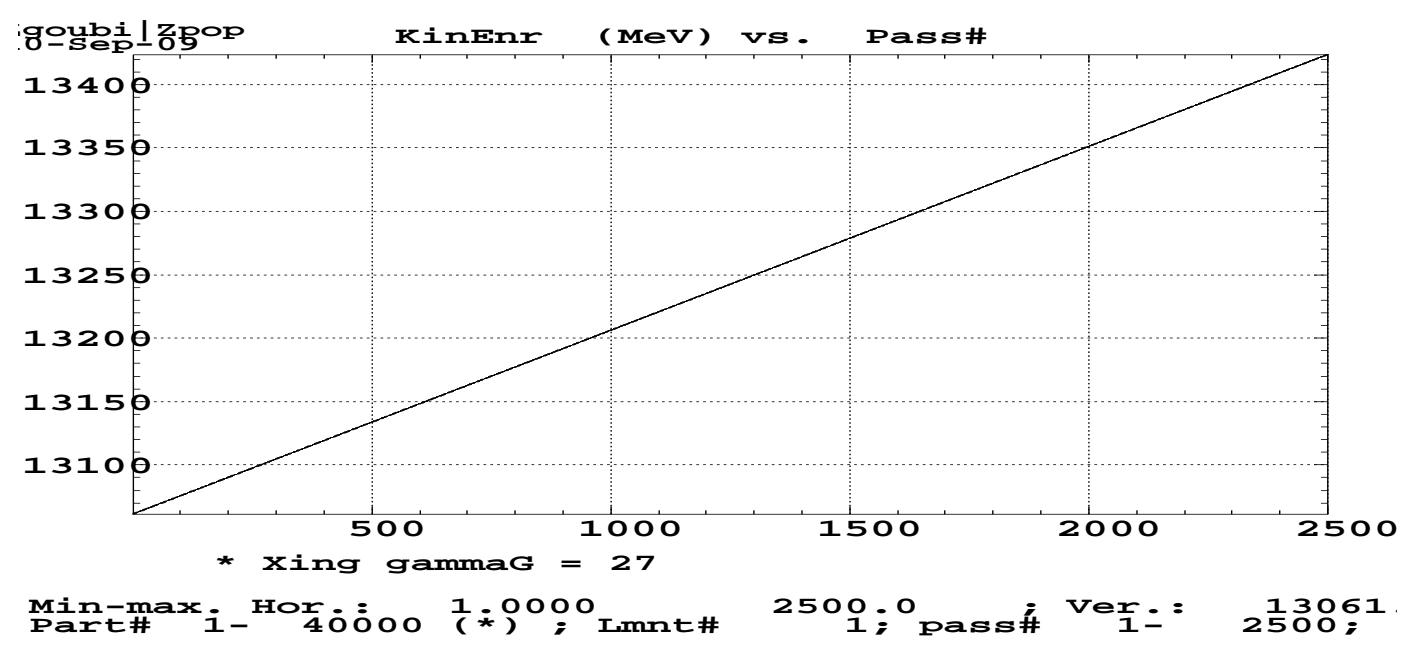

Figure 126: Kinetic E versus turn number. 


$$
\gamma G=27(13.19196 \mathbf{G e V})
$$

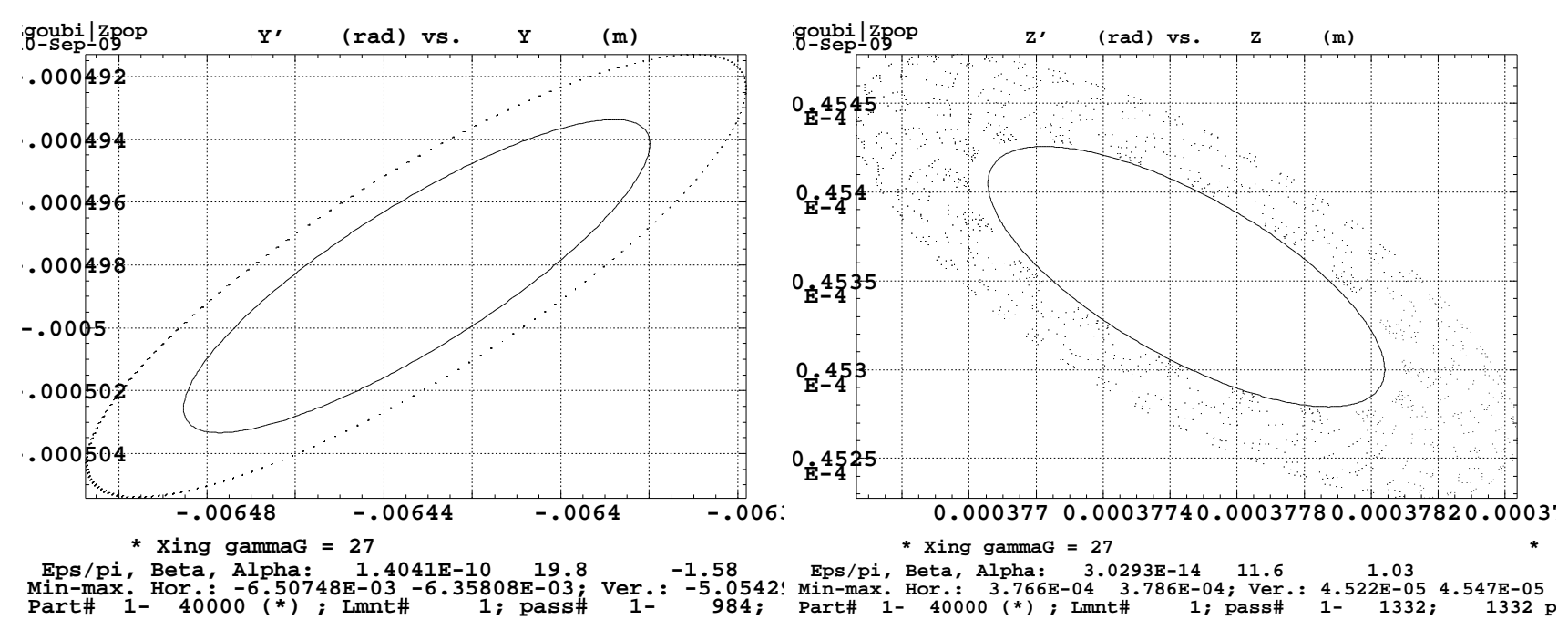

Figure 127: Left : $\mathrm{x}-\mathrm{x}^{\prime}$, right : $\mathrm{z}-\mathrm{z}^{\prime}$.

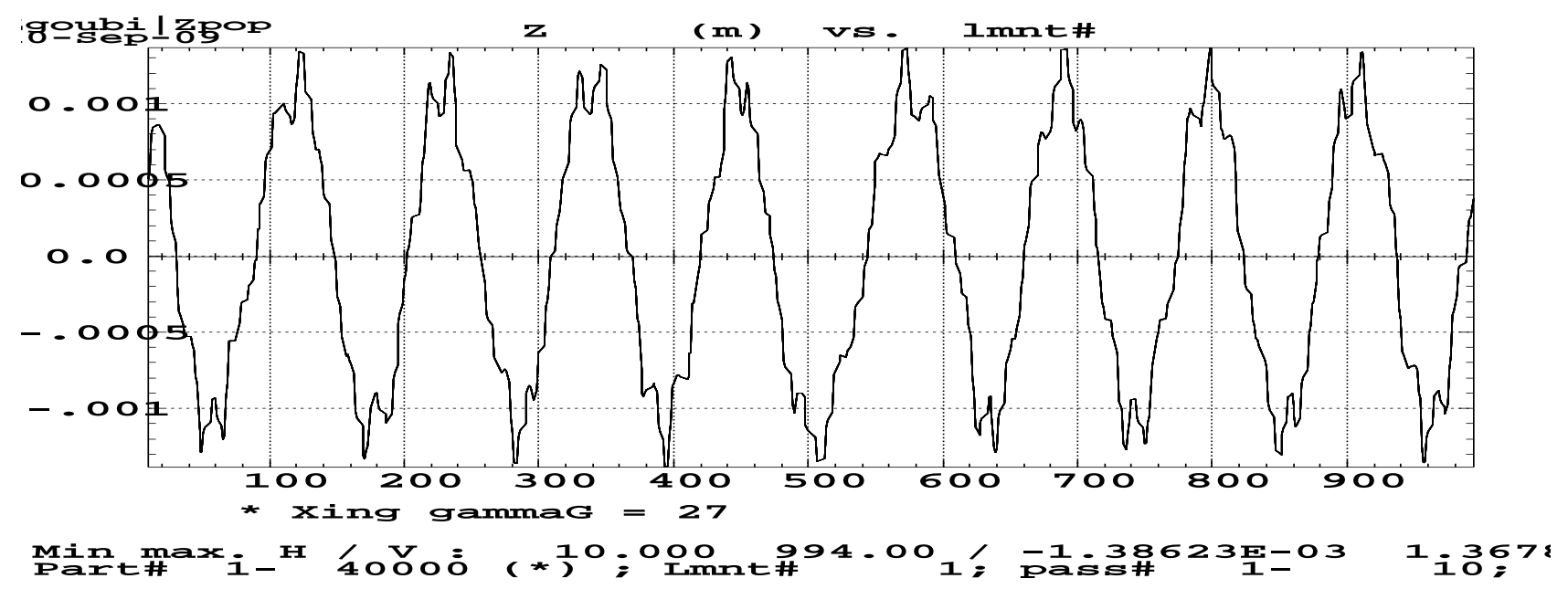

Figure 128: $\mathrm{z}$ closed orbit along the ring circumference (horizontal axis is pick-up number), 9 turns over the ring are superimposed.

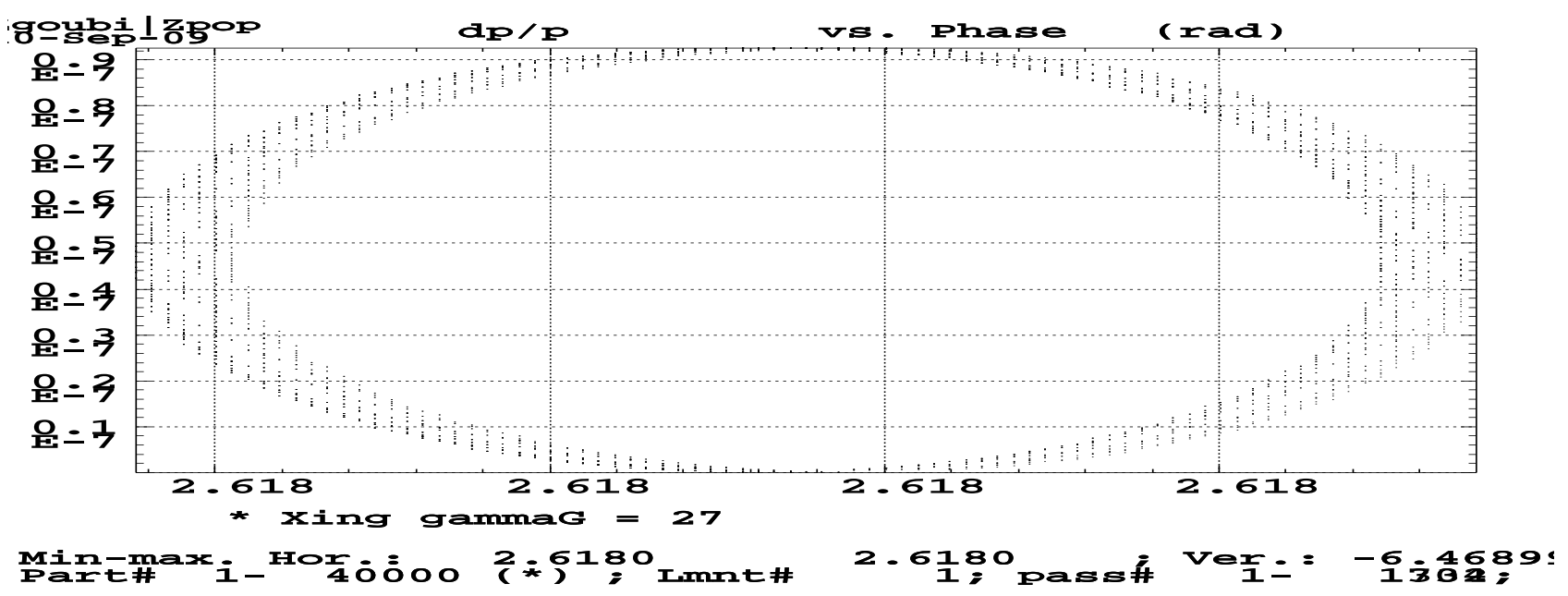

Figure 129: dp-phase. 


\subsection{6 $\gamma G=45(\mathbf{2 2 . 6 1 2 1 1} \mathbf{G e V})$}

\section{Tracking data}

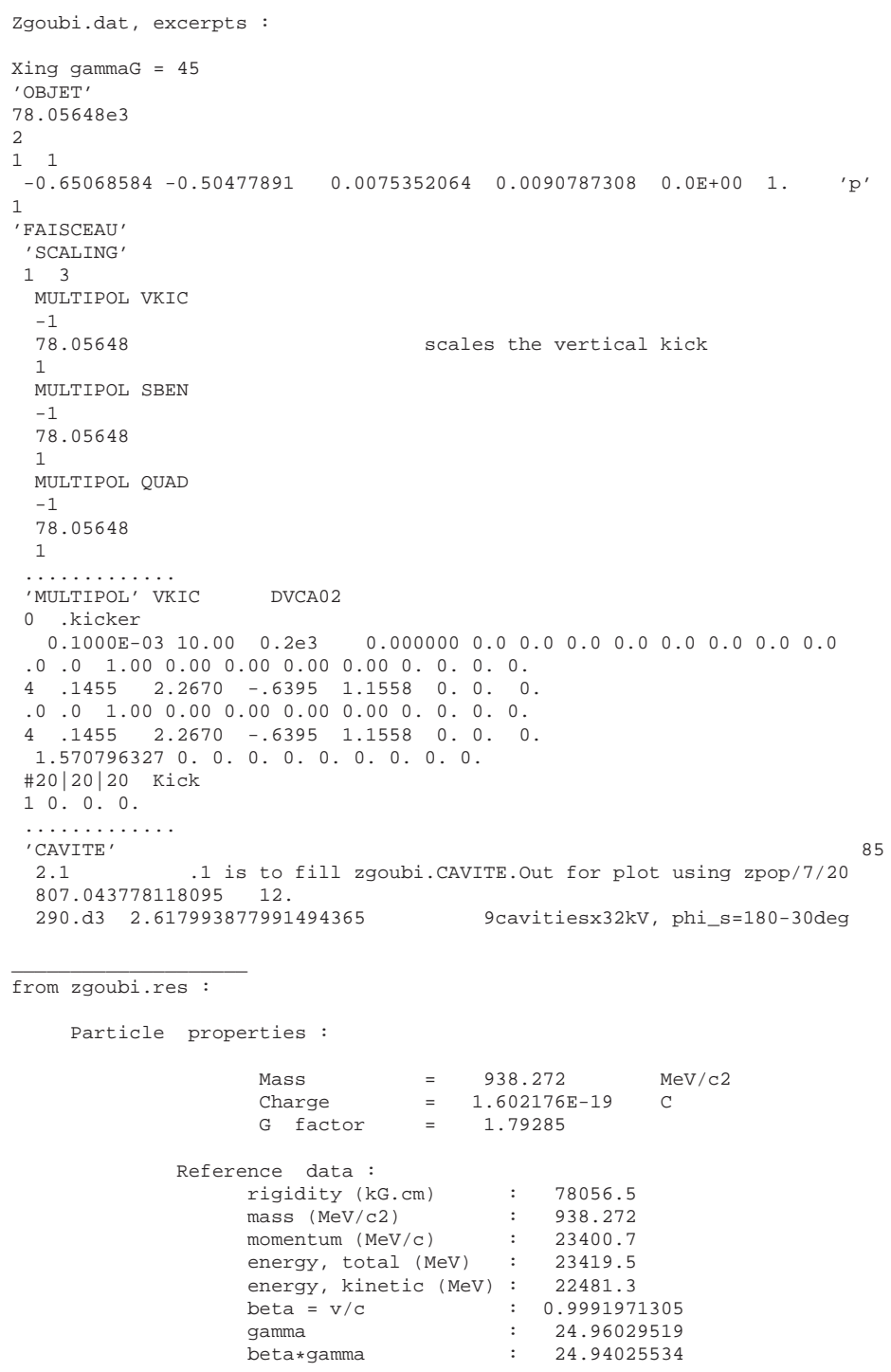

\section{Strength}

From Figs. 131, 132 one gets

$p_{\text {init }} \approx 0.999755, \quad p_{\text {final }} \approx 0.62962$

Eq. 2 yields

$$
\begin{aligned}
& A^{2}=0.2047056, \\
& \left|J_{n}\right|^{2}=5.7466214 E-06 \\
& \hat{z}_{\text {c.o. }}=0.275 \mathrm{~mm}
\end{aligned}
$$




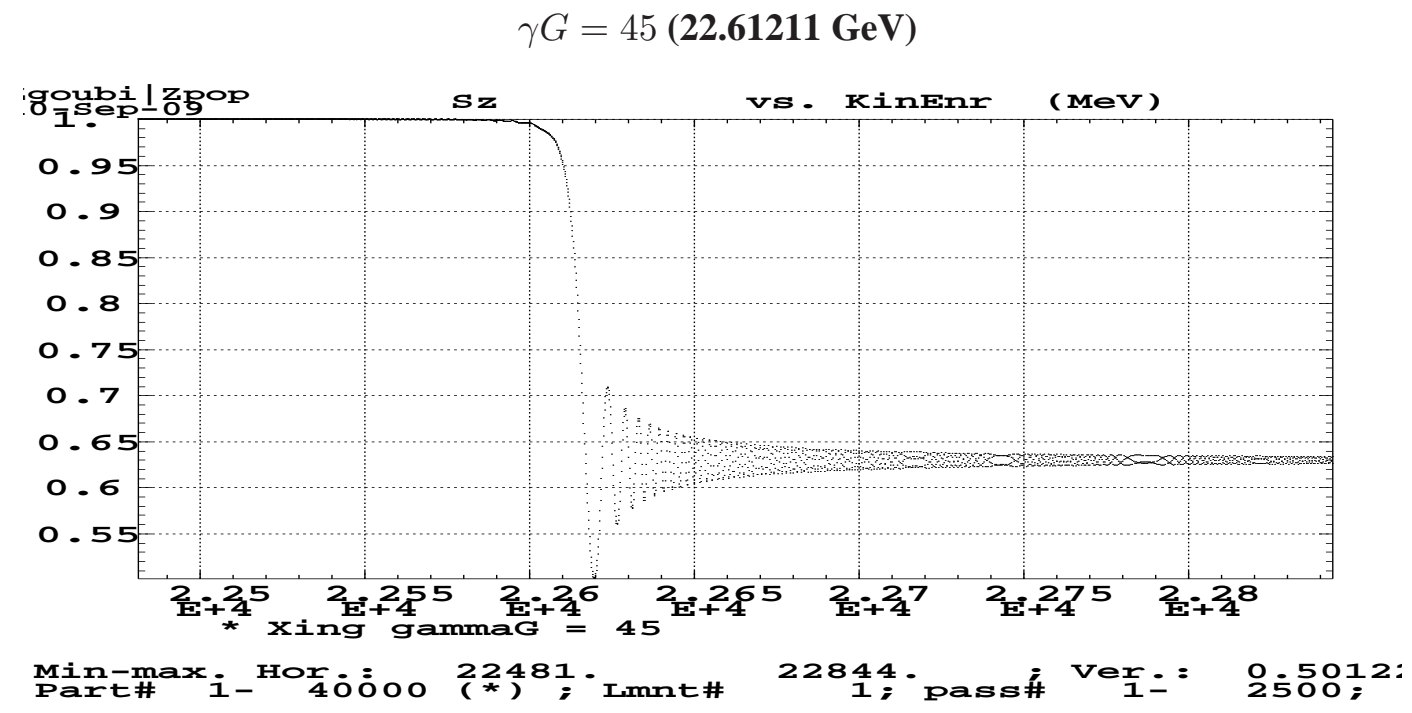

Figure 130: $S_{z}$ versus kinetic energy.

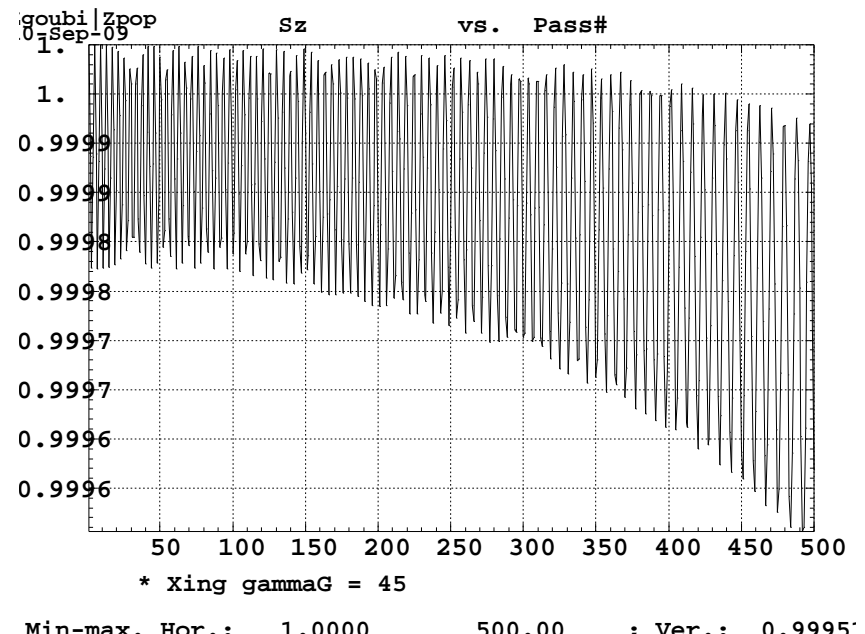

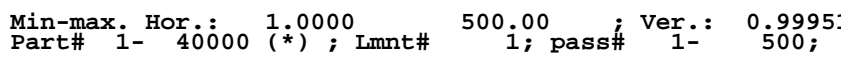

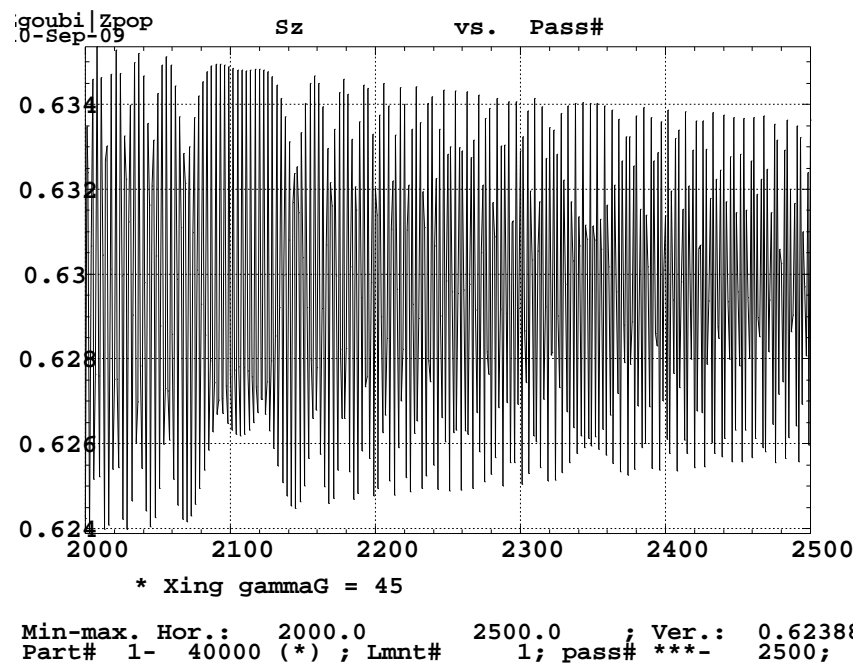

Figure 132: Zoom on final $S_{z}$.

Figure 131: Zoom on initial $S_{z}$.

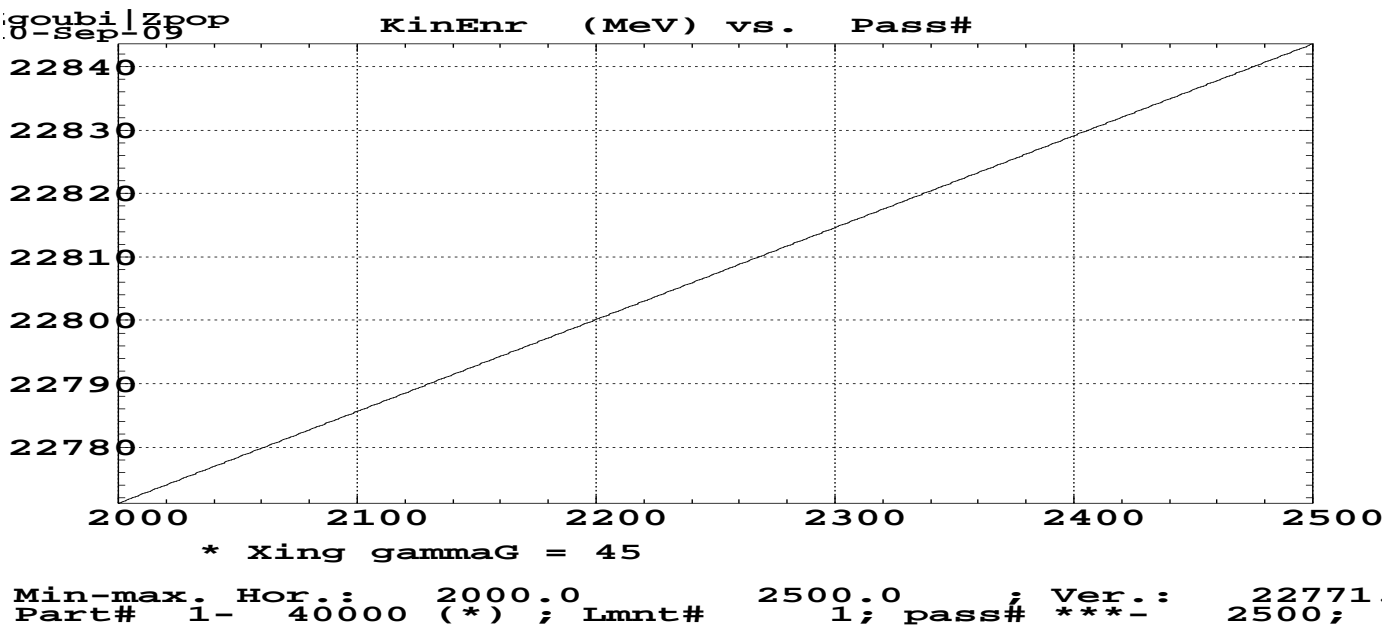

Figure 133: Kinetic E versus turn number. 


$$
\gamma G=45 \text { (22.61211 GeV) }
$$
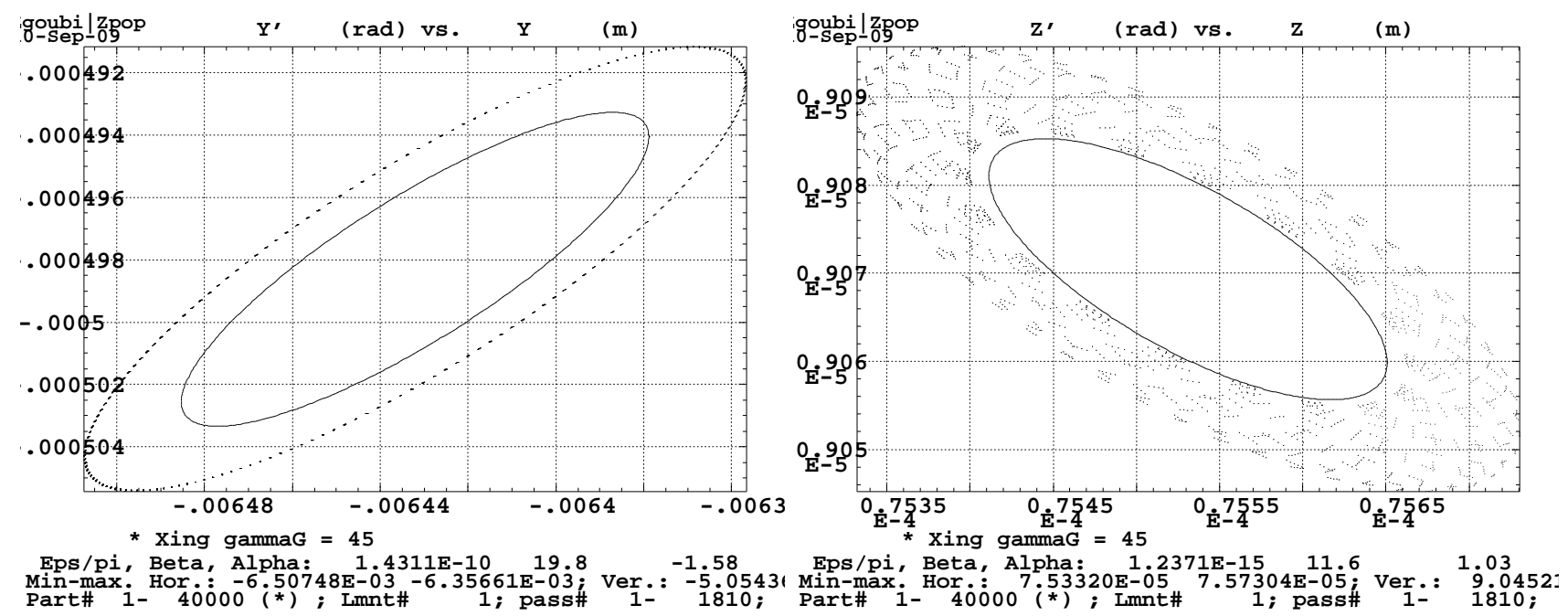

Figure 134: Left : x-x', right : z-z'.

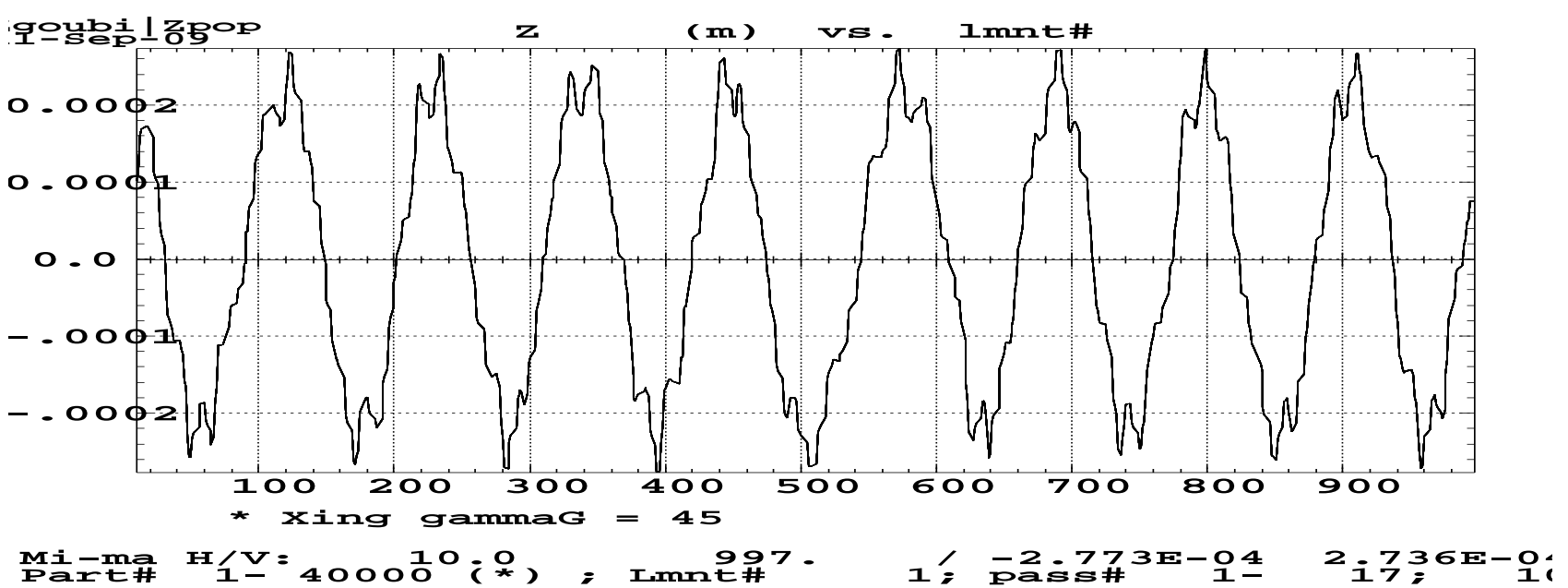

Figure 135: $\mathrm{z}$ closed orbit along the ring circumference (horizontal axis is pick-up number).

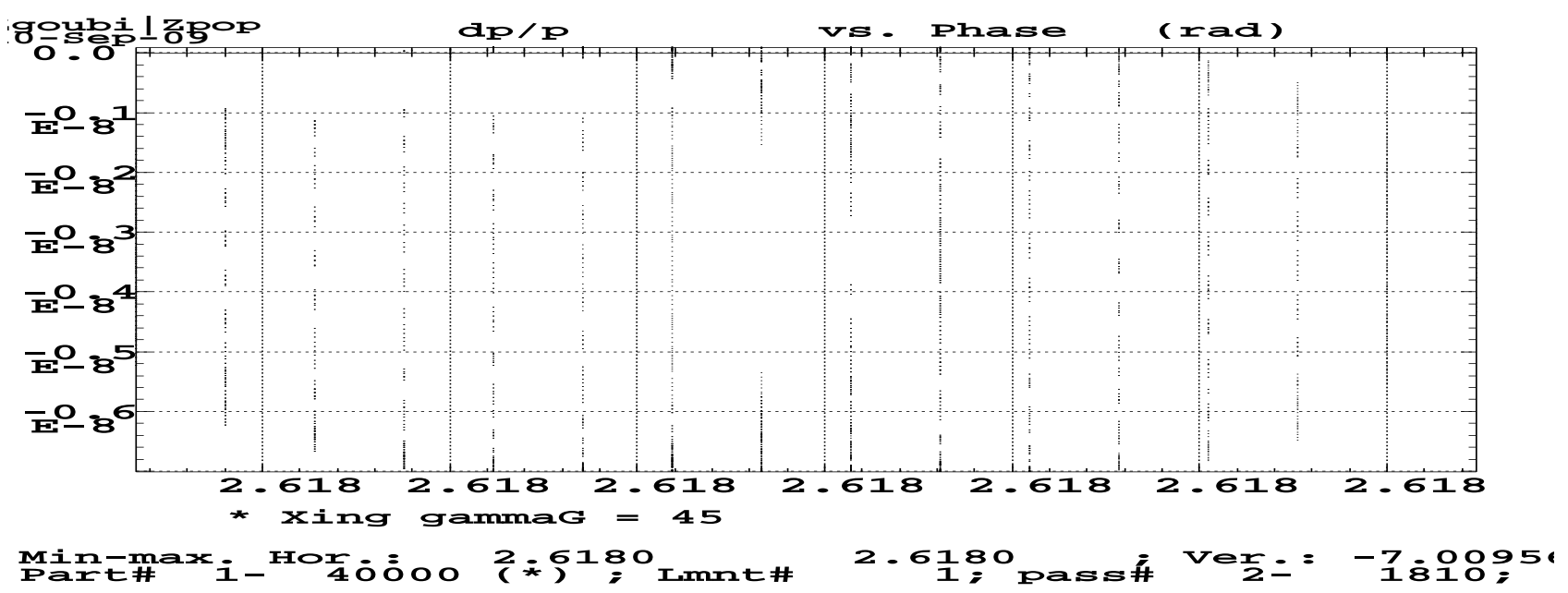

Figure 136: dp-phase. 


\section{Static neighboring of resonances}

\subsection{Intrinsic resonances}

In static mode, at distance

$$
\Delta=\text { distance to the resonance }=\gamma G-\left(n \times M-\nu_{z}\right)
$$

from an isolated spin resonance, the average value of the vertical component of an initially vertical $\vec{S}$ satisfies

$$
\bar{S}_{z}^{2}=\frac{1}{1+\left|J_{n}\right|^{2} / \Delta^{2}}, \quad \text { hence } \quad\left|J_{n}\right|^{2}=\Delta^{2} /\left(\frac{1}{1-\bar{S}_{z}^{2}}-1\right)
$$

which yields $\left|J_{n}\right|$ from a measurement of $S_{z}(\Delta)$.

In order to perform a simulation of stable precession of $\vec{S}$ around a fixed axis, test particles are launched at various, fixed, energies $\gamma=\left(\Delta+\left(n \times M-\nu_{z}\right)\right) / G$ (hence various $\left.\Delta\right)$, on non-zero vertical invariant $\epsilon_{z}$, on chromatic horizontal closed orbit corresponding to that energy and with initial $\vec{S} \equiv \vec{S}_{z}$ polarization.

The matching of the $z$-projection of the ray-traced $S_{z}$ component with $\bar{S}_{z}$, Eq. above, delivers best $\nu_{z}$ and $\epsilon$ values, these are displayed in Tab. 7 page 70 .

\subsection{1 $\gamma G=\nu_{z}(3.648013 \mathrm{GeV})$}

\section{Strengths}

Resonance strengths and vertical tunes are derived from the numerical simulation results by matching of numerical $\bar{S}_{z}(\gamma G)$ using Eq. 4, page 10.

This yields the results in Tab. 6 .

Table 6: Dependence of resonance strength squared $\left(\left|J_{n}\right|^{2}\right)$ on vertical invariant $\left(\epsilon_{z}\right) . \quad \nu_{z}$ compares fairly well with Fourier analysis of particle motion on $\epsilon_{z}$ invariant.

$\begin{array}{ccccc}\epsilon_{z} / \pi & \nu_{z} & \nu_{z} & \left|J_{n}\right|^{2} & \left|J_{n}\right|^{2} / \epsilon_{z} / \pi \\ \left(10^{-6} \mathrm{~m} . \mathrm{rad}\right) & \text { (Fourier) } & \text { (fit) } & \left(10^{-5}\right) & \\ 0.00188 & & 8.7634 & 0.07406 & 390 \\ 0.0164 & & 8.7630 & 0.7458 & 455\end{array}$




$$
\gamma G=\nu_{z}(3.648013 \mathrm{GeV})-\epsilon_{z} / \pi=0.001910^{-6}
$$

\section{Tracking data}

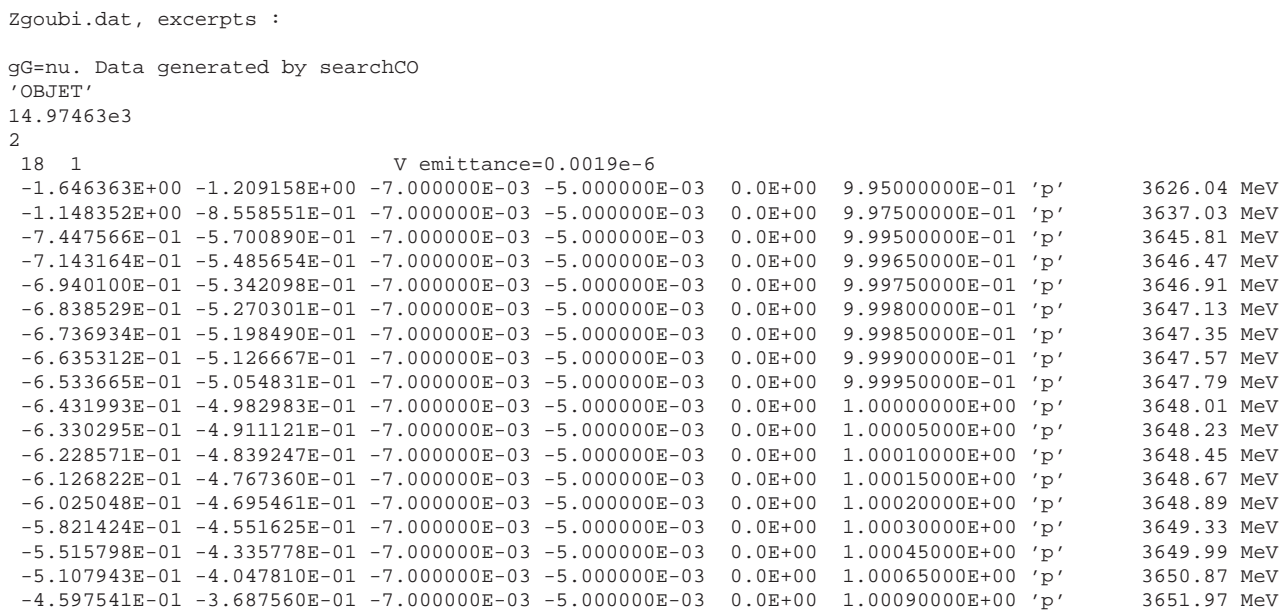

Checking computation results : for the 20 particles above, from 1200 turns analysis, their $\mathrm{H}$ closed orbits, fractional tunes (Fourier analysis) and complements to $1, \mathrm{H}$ and $\mathrm{V}$ invariant values, energy :

\begin{tabular}{|c|c|c|c|c|c|c|c|c|c|c|}
\hline $\mathrm{xCO}$ & $x^{\prime} \mathrm{CO}$ & $\mathrm{XNU}$ & ZNU & $1-\mathrm{XNU}$ & $1-\mathrm{ZNU}$ & \multicolumn{3}{|c|}{ epsilon/pi_x, z, 1} & time (mus) & $(\mathrm{MeV})$ \\
\hline $1.646363 \mathrm{E}-02$ & $-1.209158 \mathrm{E}-03$ & 184293 & 243681 & .815707 & 0.756319 & $1.983219 \mathrm{E}-17$ & $9.463248 \mathrm{E}-10$ & $1.078902 \mathrm{E}-07$ & 0.00000 & 3626.04 \\
\hline$-1.148352 \mathrm{E}-02$ & $-8.558569 \mathrm{E}-04$ & 0.236103 & 0.240276 & 0.763897 & 0.759724 & $6.203007 \mathrm{E}-18$ & $9.437355 \mathrm{E}-10$ & $7.235493 \mathrm{E}-08$ & 1.37518 & 3637.03 \\
\hline$-7.447570 \mathrm{E}-03$ & $-5.700879 \mathrm{E}-04$ & 277672 & 237293 & .722328 & 0.762707 & $5.720399 \mathrm{E}-18$ & $9.416535 \mathrm{E}-10$ & $1.039773 \mathrm{E}-08$ & 1.83348 & 645.81 \\
\hline $43173 E-03$ & $-5.485666 \mathrm{E}-04$ & 80750 & 237144 & 719250 & .762856 & $51323 E-18$ & $9.414963 \mathrm{E}-10$ & $02687 \mathrm{E}-08$ & 265 & \\
\hline$-6.940089 \mathrm{E}-03$ & $-5.342087 \mathrm{E}-04$ & 0.282798 & 0.236887 & 0.717202 & 0.763113 & $4.297505 \mathrm{E}-18$ & $9.413920 \mathrm{E}-10$ & $5.285475 \mathrm{E}-08$ & 016 & 91 \\
\hline$-6.838527 \mathrm{E}-03$ & $-5.270293 \mathrm{E}-04$ & 283828 & .236814 & 0.716172 & 0.763186 & $18882 \mathrm{E}-18$ & $9.413402 \mathrm{E}-10$ & $45 E-08$ & 83 & 13 \\
\hline $942 \mathrm{E}-03$ & $-5.198491 \mathrm{E}-04$ & 4866 & 236765 & 134 & 235 & -18 & -10 & & & \\
\hline$-6.635313 \mathrm{E}-03$ & $-5.126674 \mathrm{E}-04$ & 85919 & 236722 & 0.714081 & 0.763278 & $E-18$ & $366 \mathrm{E}-10$ & -08 & 41 & 7 \\
\hline$-6.533653 E-03$ & $-5.054814 \mathrm{E}-04$ & .287004 & .236668 & 0.712996 & 0.763332 & $E-18$ & $43 \mathrm{E}-10$ & $E-08$ & & 7.79 \\
\hline$-6.432005 E-03$ & $-4.982988 E-04$ & 085 & 881 & 15 & 419 & -18 & -10 & 08 & & \\
\hline$-6.330296 \mathrm{E}-03$ & $-4.911123 \mathrm{E}-04$ & 0.289138 & 0.236422 & 0.710862 & 0.763578 & $3.260158 \mathrm{E}-18$ & $9.410789 \mathrm{E}-10$ & $1.516312 \mathrm{E}-08$ & 2.50016 & 3648.23 \\
\hline$-6.228566 \mathrm{E}-03$ & $-4.839242 \mathrm{E}-04$ & 0.290175 & 0.236333 & 0.709825 & 0.763667 & $30 \mathrm{E}-18$ & $9.410263 \mathrm{E}-10$ & $E-08$ & 2.52099 & 48.45 \\
\hline$-6.126822 \mathrm{E}-03$ & $-4.767353 \mathrm{E}-04$ & 291205 & 279 & 0.70 & 21 & $E-18$ & -10 & 08 & & 3.67 \\
\hline$-6.025061 E-03$ & $-4.695472 \mathrm{E}-04$ & 292230 & 236235 & 0.70 & 0.763765 & $2 \mathrm{E}-18$ & $9.409217 \mathrm{E}-10$ & $36 \mathrm{E}-08$ & 72 & 8.89 \\
\hline$-5.821414 \mathrm{E}-03$ & $-4.551620 \mathrm{E}-04$ & 0.294278 & 0.236110 & 0.705722 & 0.763890 & $08 \mathrm{E}-18$ & $9.408175 \mathrm{E}-10$ & $35 \mathrm{E}-08$ & 2.56681 & 3649.33 \\
\hline$-5.515801 \mathrm{E}-03$ & $-4.335775 \mathrm{E}-04$ & .297380 & 235790 & 0.702620 & 0.764210 & $21 \mathrm{E}-18$ & $618 \mathrm{E}-10$ & $81 \mathrm{E}-08$ & & $\$ 49.99$ \\
\hline$-5.107945 \mathrm{E}-03$ & $-4.047817 \mathrm{E}-04$ & .301655 & 235489 & 345 & 64511 & $E-18$ & $511 \mathrm{E}-10$ & 1. & 35 & 0.87 \\
\hline$-4.597556 \mathrm{E}-03$ & $-3.687562 \mathrm{E}-04$ & .306793 & 0.235137 & 0.693207 & 0.764863 & $1.077477 \mathrm{E}-17$ & $9.401906 \mathrm{E}-10$ & $3.335808 \mathrm{E}-08$ & 2.59732 & 3651.97 \\
\hline
\end{tabular}


$\gamma G=\nu_{z}(3.648013 \mathrm{GeV})-\epsilon_{z} / \pi=0.001910^{-6}$

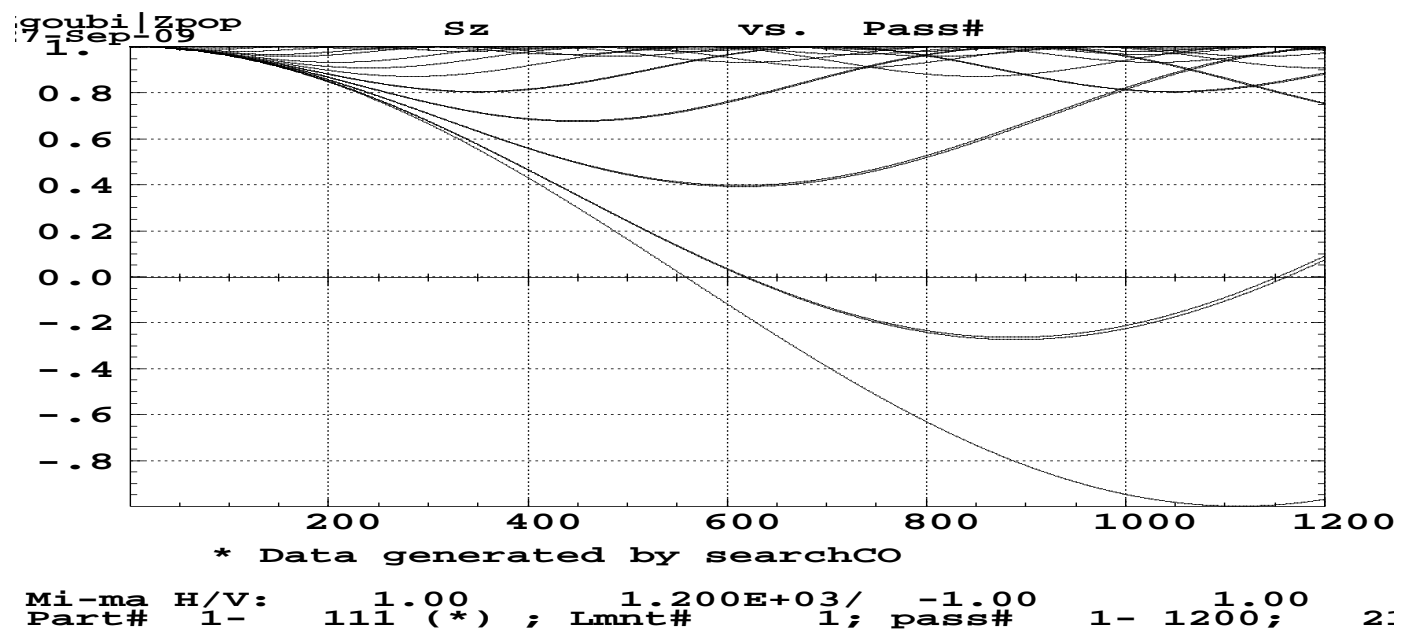

Figure 137: $S_{z}$ versus turn number for various distances to the resonance.
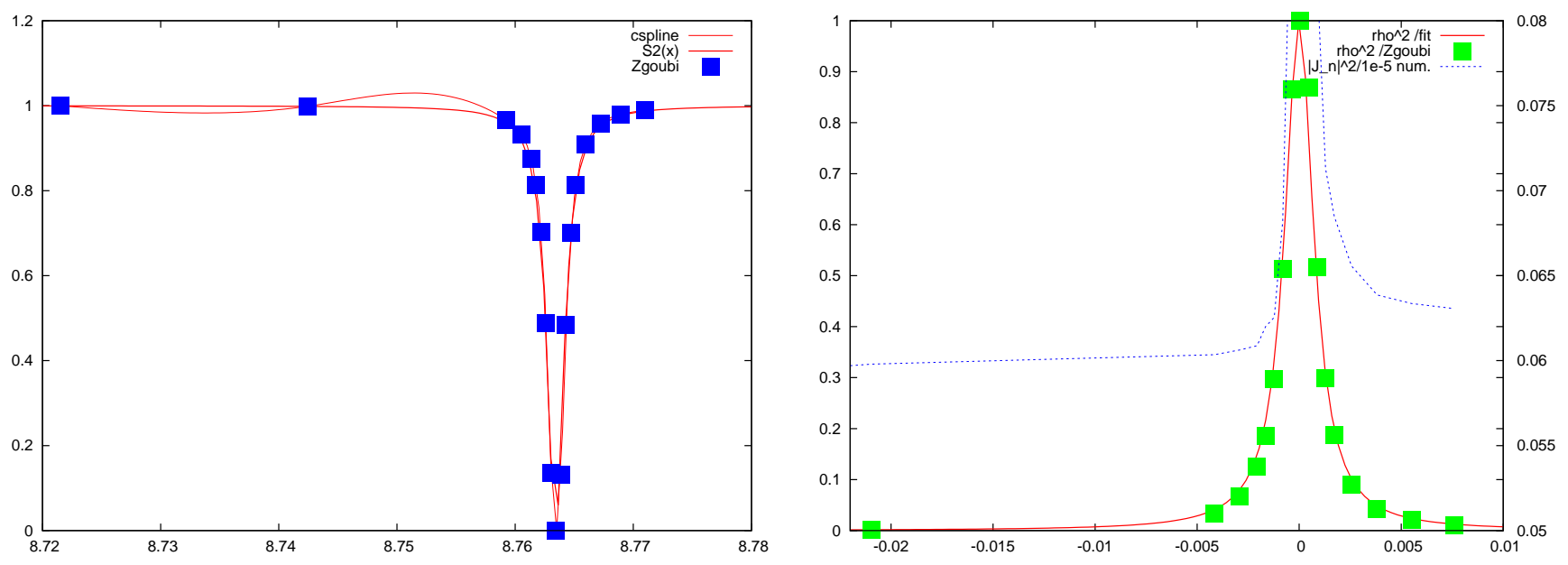

Figure 138: Matching $S_{z}^{2}(\Delta)$ (left, Eq. 4) and $\rho^{2}(\Delta)$ (right, Eq. 5) The right plot also shows $\left|J_{n}\right|^{2}$ from numerical data (with expectable lack of accuracy in the region $\Delta \rightarrow 0$ ).
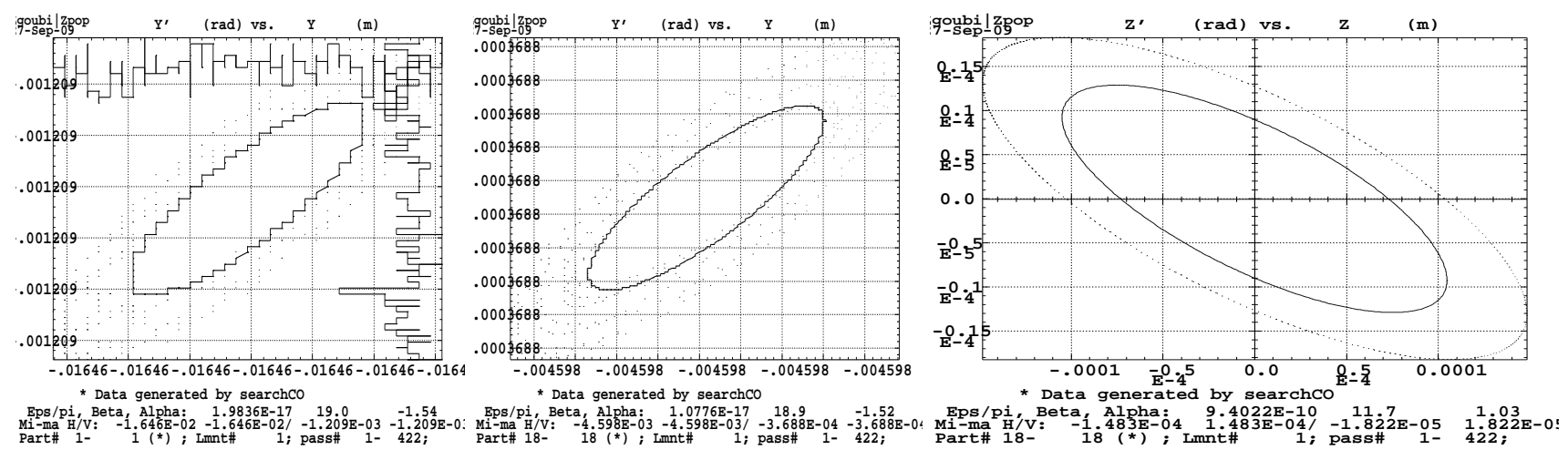

Figure 139: Left : $\mathrm{x}-\mathrm{x}$ ' of particles at min. and max. $p / p_{0}$. Right : z-z' (all particles superimposed). 


$$
\gamma G=\nu_{z}(3.648013 \mathrm{GeV})-\epsilon_{z} / \pi=0.01710^{-6}
$$

\section{Tracking data}

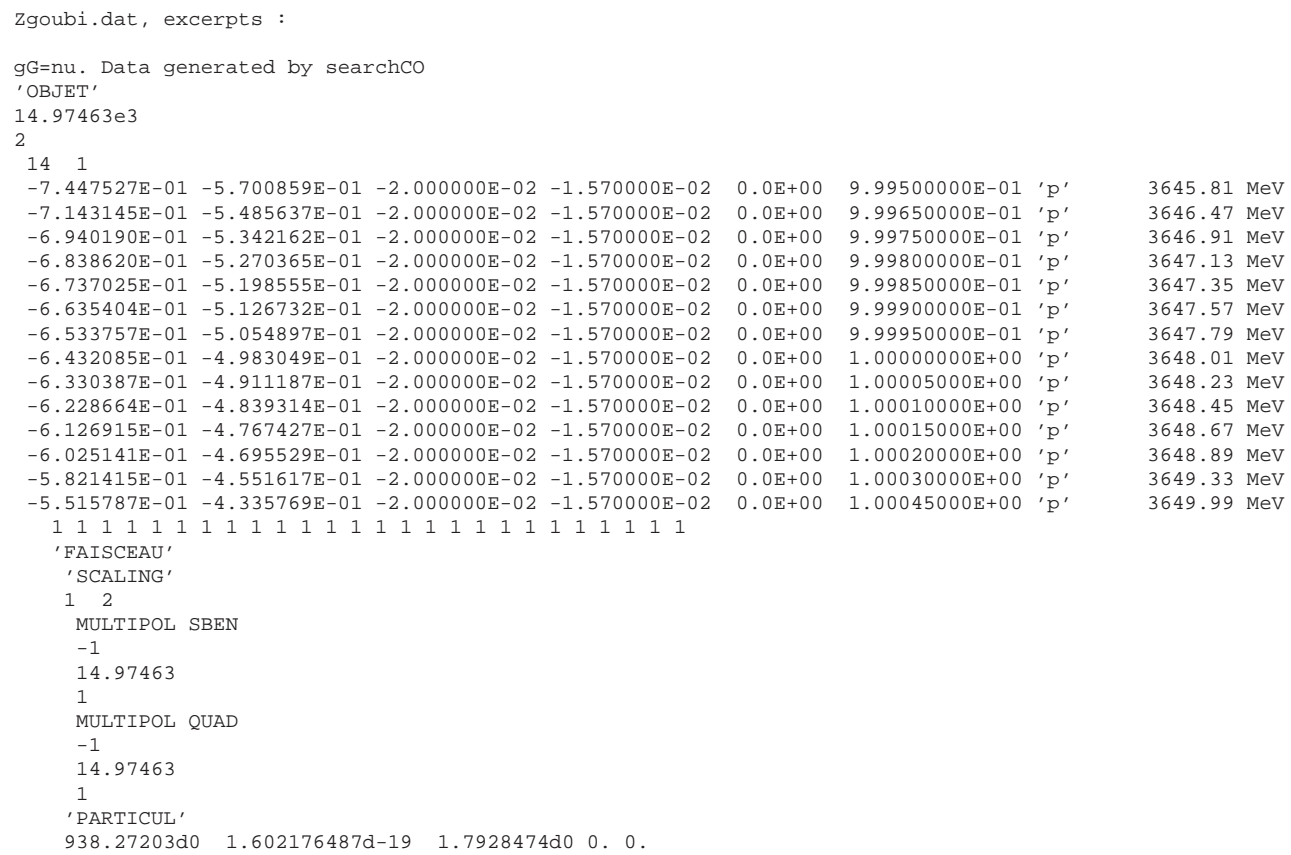

Checking computation results : for the 20 particles above, from 200 turns analysis, their $\mathrm{H}$ closed orbits, fractional tunes (Fourier analysis) and complements to $1, \mathrm{H}$ and $\mathrm{V}$ invariant values, energy : 
$\gamma G=\nu_{z}(3.648013 \mathrm{GeV})-\epsilon_{z} / \pi=0.01710^{-6}$

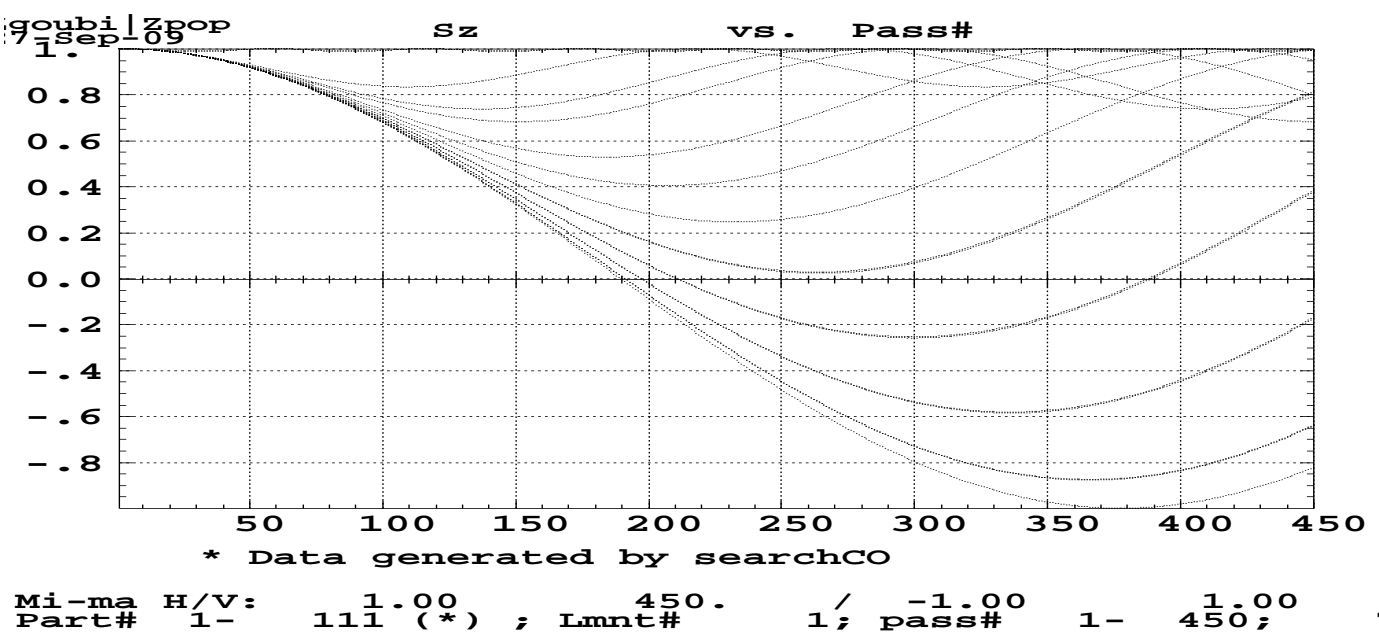

Figure 140: $S_{z}$ versus turn number for various distances to the resonance.
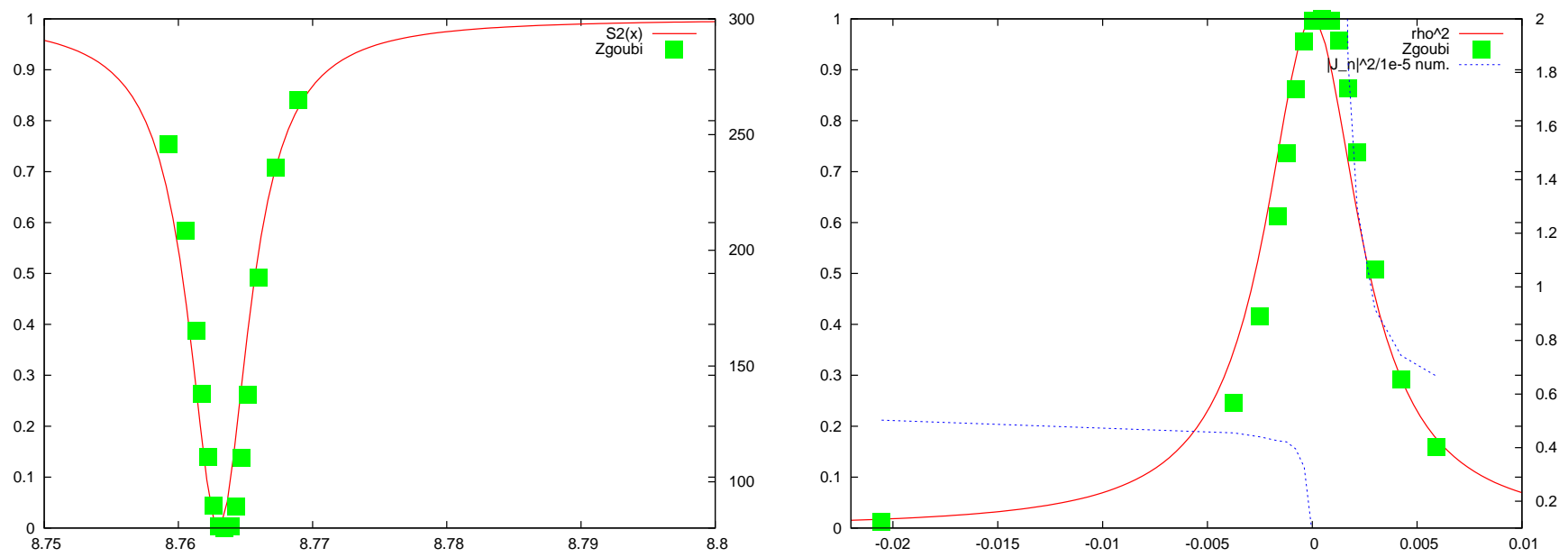

Figure 141: Matching $S_{z}^{2}(\Delta)$ (left, Eq. 4) and $\rho^{2}(\Delta)$ (right, Eq. 5) The right plot also shows $\left|J_{n}\right|^{2}$ from numerical data (with expectable lack of accuracy in the region $\Delta \rightarrow 0$ ).
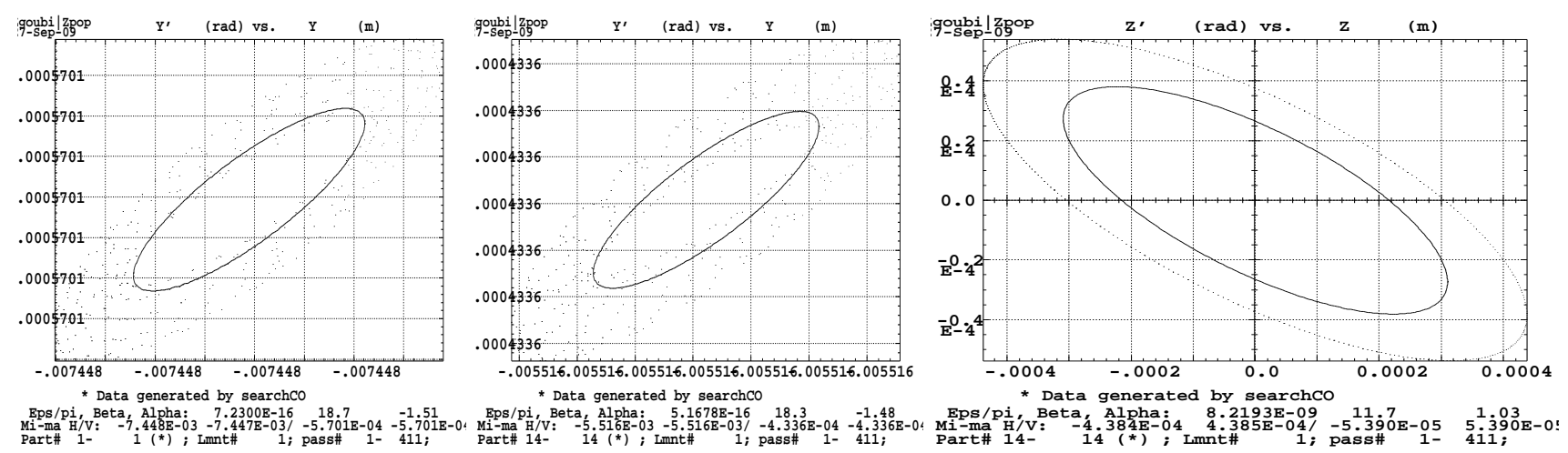

Figure 142: Left : $\mathrm{x}-\mathrm{x}$ ' of particles at min. and max. $p / p_{0}$. Right : z-z' (all particles superimposed). 


\subsection{2 $\gamma G=48-\nu_{z}(\mathbf{1 9 . 5 9 5 8 5} \mathbf{G e V})$}

\section{Strengths}

Resonance strengths and vertical tunes are derived from the numerical simulation results by matching of numerical $\bar{S}_{z}(\gamma G)$ using Eq. 4, page 10 .

This yields the results in Tab. 7. $\nu_{z}$ compares fairly well with Fourier analysis of particle motion on $\epsilon_{z}$ invariant. $\left|J_{n}\right|^{2} / \epsilon_{z} / \pi$ appears to be but weakly dependent on $\epsilon_{z}$ in agreement with the results of resonance crossing, Sec. 4.1 .7 (Tab. 4 page 35), and with Eq. 10.

Table 7: Dependence of resonance strength squared, $\left|J_{n}\right|^{2}$, on vertical invariant $\left(\epsilon_{z}\right)$.

$\begin{array}{ccccc}\epsilon_{z} / \pi & \nu_{z} & \nu_{z} & \left|J_{n}\right|^{2} & \left|J_{n}\right|^{2} / \epsilon_{z} / \pi \\ \left(10^{-6} \mathrm{~m} . \text { rad }\right) & (\text { Fourier }) & \text { (fit) } & \left(10^{-5}\right) & \\ 0.125 & .774 & 8.7740 & 0.354 & 27 \\ 2 & .774 & 8.77 & 6.249 & 31\end{array}$




$$
\gamma G=48-\nu_{z}(19.59585 \mathrm{GeV})-\epsilon_{z} / \pi=0.12510^{-6}
$$

\section{Tracking data}

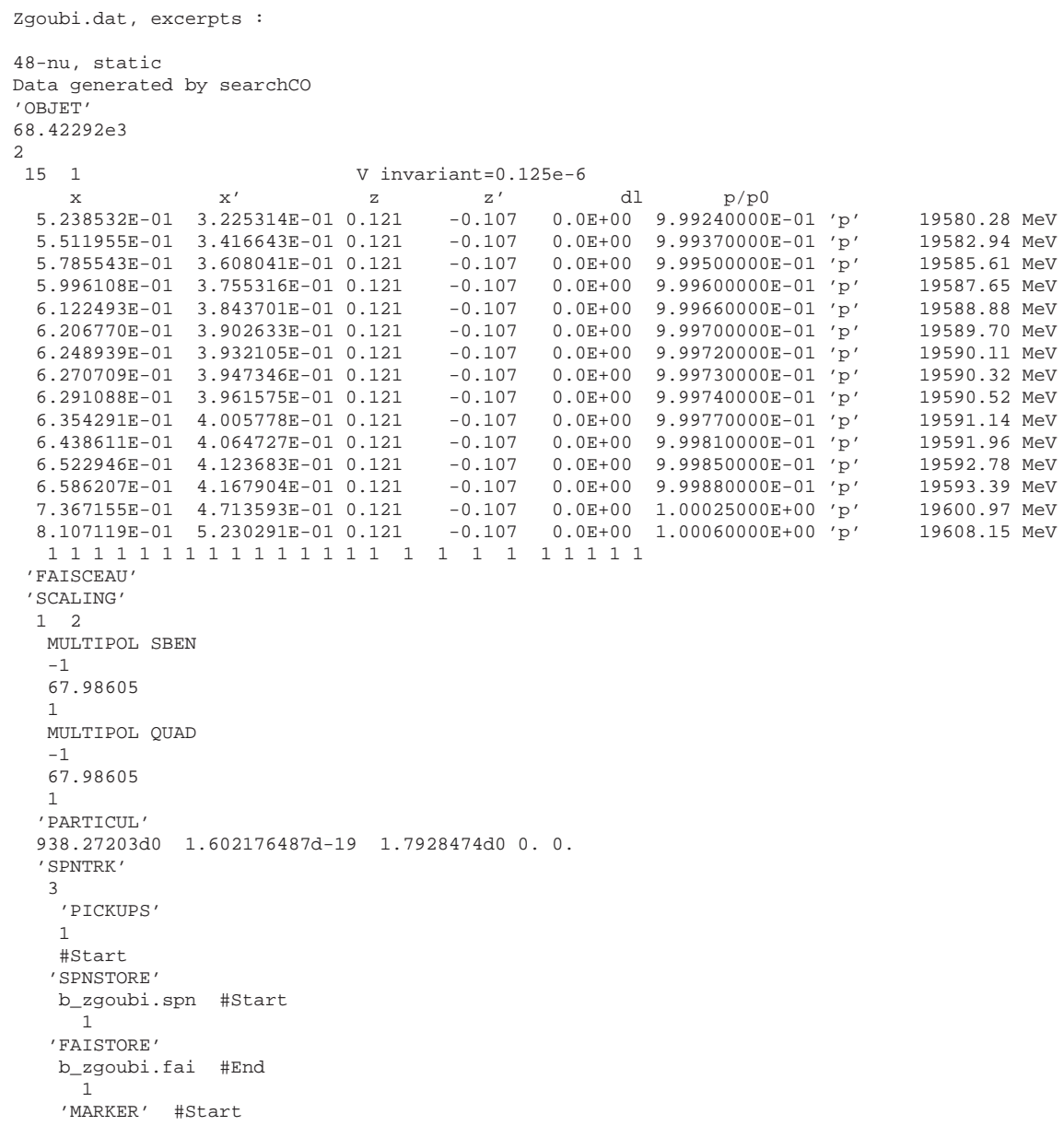

Checking computation results : for the 15 particles above, , from 400 turns analysis, their $\mathrm{H}$ closed orbits, fractional tunes and complements to $1, \mathrm{H}$ and $\mathrm{V}$ invariant values, energy :

\begin{tabular}{|c|c|c|c|c|c|c|c|c|c|c|}
\hline $\mathrm{CO}$ & $\mathrm{CO}$ & $\mathrm{U}$ & ZNU & $1-\mathrm{XNU}$ & $\mathrm{U}$ & & 1 & & us) & V) \\
\hline $5.238426 \mathrm{E}-03$ & $225236 \mathrm{E}-04$ & 452922 & 226474 & .547078 & 773526 & $944626 \mathrm{E}-15$ & $260669 \mathrm{E}-08$ & $8.490941 \mathrm{E}-08$ & 2.69504 & 580.3 \\
\hline $45 \mathrm{E}-03$ & $6666 \mathrm{E}-04$ & 2434 & 6220 & 47566 & 73780 & תר & & $53 \mathrm{E}-08$ & & \\
\hline $9 \mathrm{E}-03$ & $964 \mathrm{E}-04$ & & & & & & & & & \\
\hline $6270 E-03$ & $5278 \mathrm{E}-04$ & 51525 & 0.225760 & 548475 & & $3 E-15$ & & & & \\
\hline & & & & & & & & & & \\
\hline 3 & 4 & 1102 & 5 & 98 & 0.7 & & & & & \\
\hline & & & & & & & & & & \\
\hline & & & & & & & & & & \\
\hline-03 & -04 & 0930 & 0.225473 & 70 & 0.77 & -15 & & 08 & & \\
\hline & & & & & & & & & & \\
\hline & & & & & & & & & & \\
\hline 6.5 & 4 & 3 & 225252 & & 0.7 & -15 & & & & \\
\hline 6.5 & -04 & 343 & 25196 & 57 & 0.7 & 15 & & & & \\
\hline & 然 & & & & & -15 & & 8 & & \\
\hline $12 \mathrm{E}-03$ & $230102 \mathrm{E}-04$ & 1.447343 & 223718 & 552657 & 776282 & $407776 \mathrm{E}-15$ & $6.261412 \mathrm{E}-08$ & $4.670097 \mathrm{E}-08$ & 2.69509 & 9608.1 \\
\hline
\end{tabular}


$\gamma G=48-\nu_{z}(19.59585 \mathrm{GeV})-\epsilon_{z} / \pi=0.12510^{-6}$

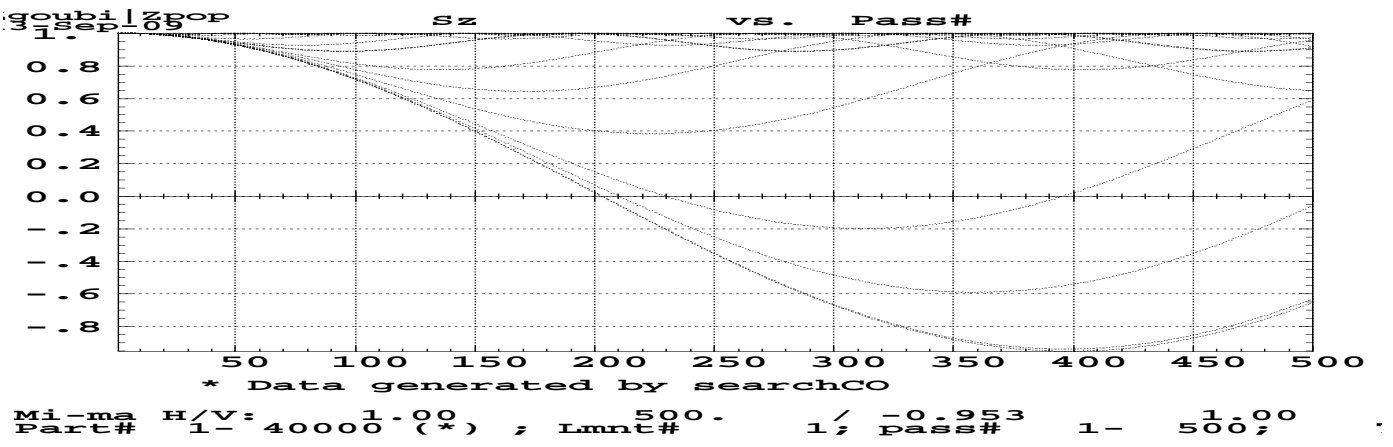

Figure 143: $S_{z}$ versus turn number for various distances to the resonance.
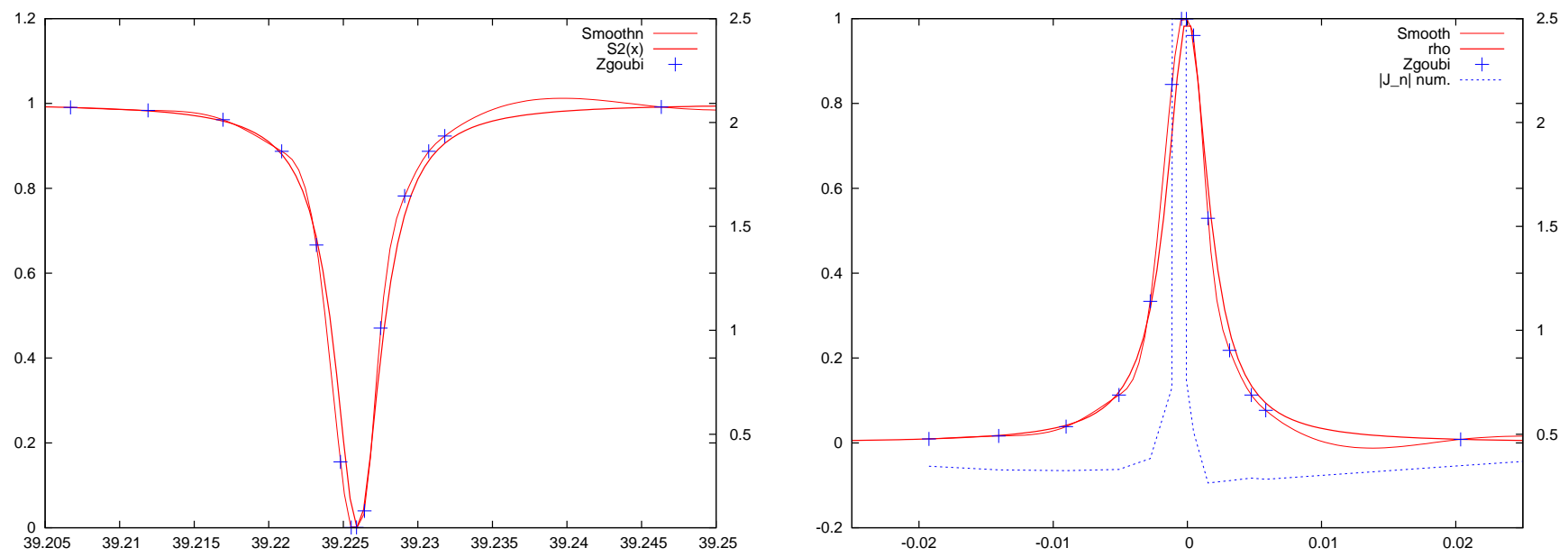

Figure 144: Matching $S_{z}^{2}(\Delta)$ (left, Eq. 4) and $\rho^{2}(\Delta)$ (right, Eq. 5) The right plot also shows $\left|J_{n}\right|^{2}$ from numerical data (with expectable lack of accuracy in the region $\Delta \rightarrow 0$ ).
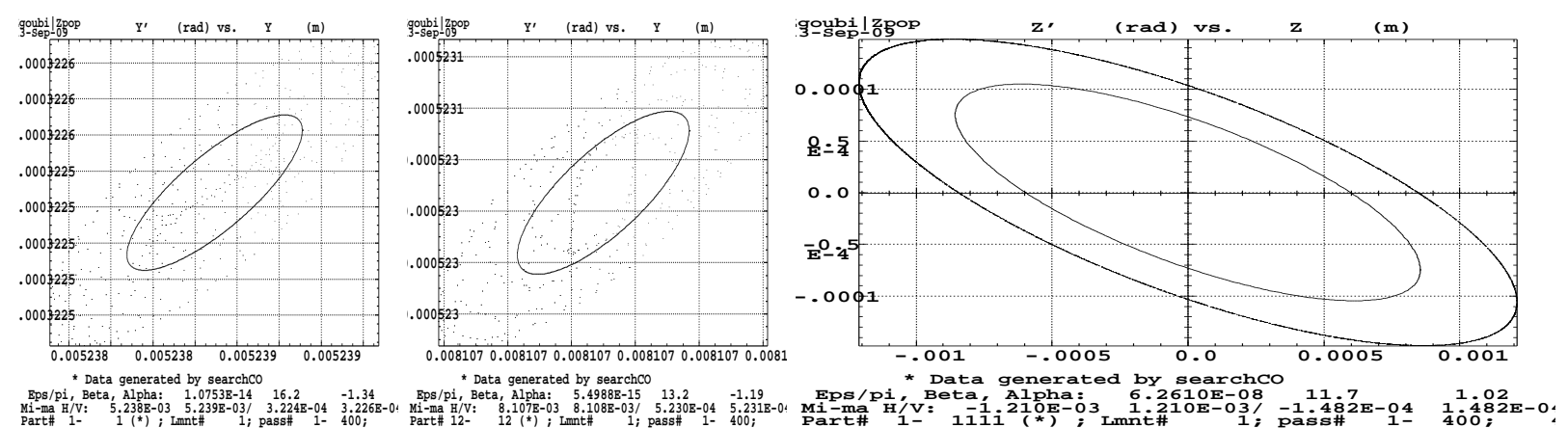

Figure 145: Left : $\mathrm{x}-\mathrm{x}^{\prime}$ of particles at min. and max. $p / p_{0}$. Right : z-z' (all particles superimposed). 
$\gamma G=48-\nu_{z}(19.59585 \mathrm{GeV})-\epsilon_{z} / \pi=210^{-6}$

\section{Tracking data}

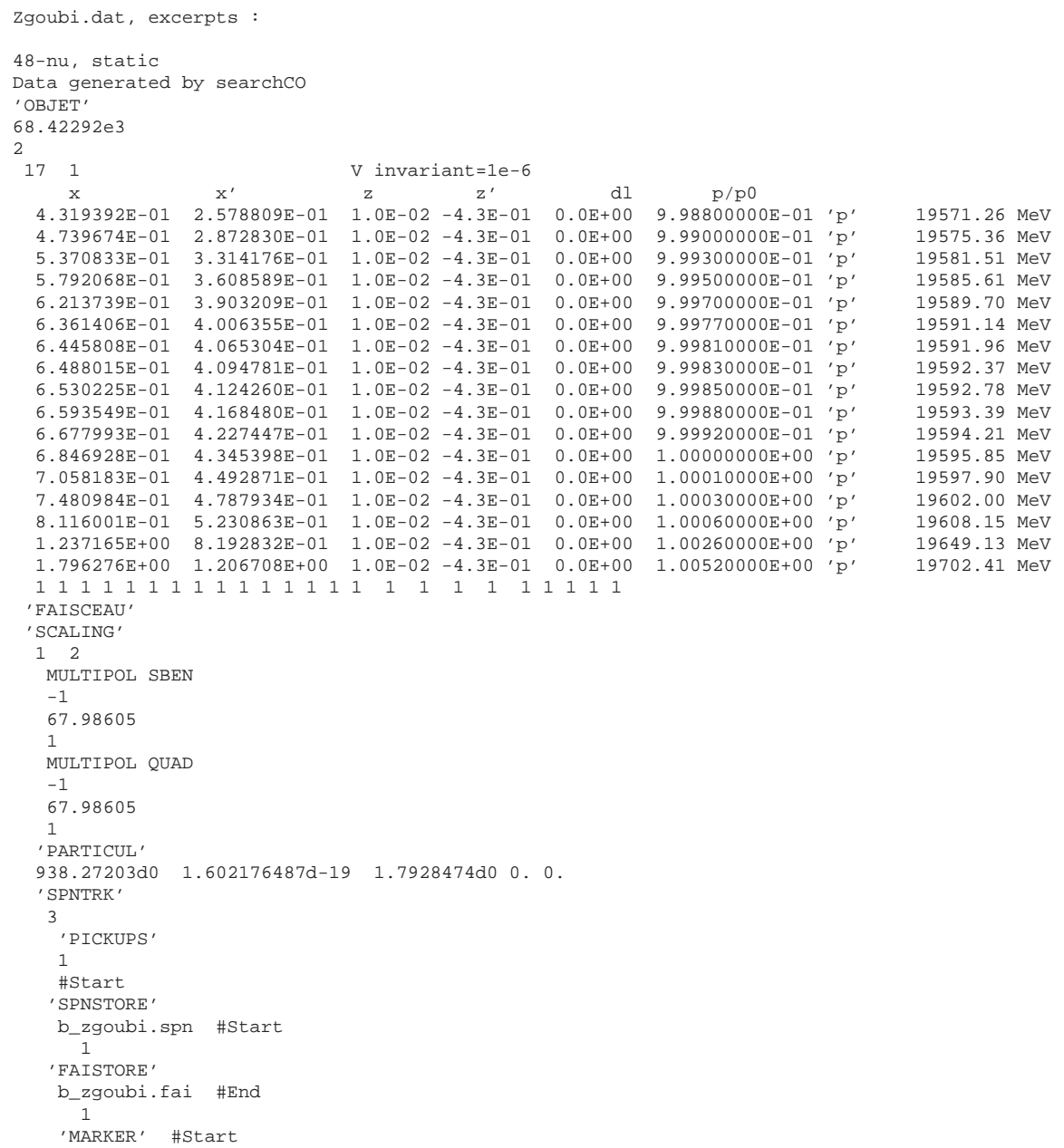

Checking computation results : for the 17 particles above, from 400 turns analysis, their $\mathrm{H}$ closed orbits, fractional tunes and complements to $1, \mathrm{H}$ and $\mathrm{V}$ invariant values, energy :

$\begin{array}{cc}\mathrm{XCO} & \mathrm{X}^{\prime} \mathrm{CO} \\ 4.292329 \mathrm{E}-03 & 2.565613 \mathrm{E}-04 \\ 4.727305 \mathrm{E}-03 & 2.860852 \mathrm{E}-04 \\ 5.352981 \mathrm{E}-03 & 3.312343 \mathrm{E}-04 \\ 5.768571 \mathrm{E}-03 & 3.588075 \mathrm{E}-04 \\ 6.203395 \mathrm{E}-03 & 3.906452 \mathrm{E}-04 \\ 6.331626 \mathrm{E}-03 & 3.984651 \mathrm{E}-04 \\ 6.437418 \mathrm{E}-03 & 4.065558 \mathrm{E}-04 \\ 6.460069 \mathrm{E}-03 & 4.079638 \mathrm{E}-04 \\ 6.516286 \mathrm{E}-03 & 4.115880 \mathrm{E}-04 \\ 6.572890 \mathrm{E}-03 & 4.162095 \mathrm{E}-04 \\ 6.656351 \mathrm{E}-03 & 4.211746 \mathrm{E}-04 \\ 6.832306 \mathrm{E}-03 & 4.344203 \mathrm{E}-04 \\ 7.031513 \mathrm{E}-03 & 4.473885 \mathrm{E}-04 \\ 7.469509 \mathrm{E}-03 & 4.789586 \mathrm{E}-04 \\ 8.084513 \mathrm{E}-03 & 5.208150 \mathrm{E}-04 \\ 1.234425 \mathrm{E}-02 & 8.178755 \mathrm{E}-04 \\ 1.794068 \mathrm{E}-02 & 1.207013 \mathrm{E}-03\end{array}$ $\begin{array}{llll}\mathrm{XNU} & \mathrm{ZNU} & 1-\mathrm{XNU} & 1-\mathrm{ZNU}\end{array}$ $0.3973910 .2273870 .6026090 .7726132 .782303 E-12$ silon/pi_X, Z, 1 $\begin{array}{lllllll}0.401652 & 0.227005 & 0.598348 & 0.772995 & 2.813073 \mathrm{E}-12 & 9.975898 \mathrm{E}-07 & 5.660611 \mathrm{E}-09\end{array}$ $\begin{array}{llllllll}0.408019 & 0.226401 & 0.591981 & 0.773599 & 2.810842 \mathrm{E}-12 & 9.976181 \mathrm{E}-07 & 4.660611 \mathrm{E}-09\end{array}$ $\begin{array}{llllllll}0.412253 & 0.226013 & 0.587747 & 0.773987 & 2.777554 \mathrm{E}-12 & 9.976402 \mathrm{E}-07 & 1.556678 \mathrm{E}-08\end{array}$ $\begin{array}{llllllll}0.416524 & 0.225618 & 0.583476 & 0.774382 & 2.819153 \mathrm{E}-12 & 9.976524 \mathrm{E}-07 & 1.415165 \mathrm{E}-08\end{array}$ $\begin{array}{llllllll}0.418009 & 0.225463 & 0.581991 & 0.774537 & 2.803603 \mathrm{E}-12 & 9.976563 \mathrm{E}-07 & 2.830332 \mathrm{E}-09\end{array}$ $\begin{array}{llllllll}0.418836 & 0.225378 & 0.581164 & 0.774622 & 2.789871 \mathrm{E}-12 & 9.976590 \mathrm{E}-07 & 4.953084 \mathrm{E}-08\end{array}$ $\begin{array}{llllllll}0.419275 & 0.225338 & 0.580725 & 0.774662 & 2.770508 \mathrm{E}-12 & 9.976604 \mathrm{E}-07 & 4.103985 \mathrm{E}-08\end{array}$ $\begin{array}{llllllll}0.419715 & 0.225300 & 0.580285 & 0.774700 & 2.804960 \mathrm{E}-12 & 9.976619 \mathrm{E}-07 & 6.368254 \mathrm{E}-08\end{array}$ $\begin{array}{llllllll}0.420325 & 0.225244 & 0.579675 & 0.774756 & 2.806966 \mathrm{E}-12 & 9.976642 \mathrm{E}-07 & 5.236122 \mathrm{E}-08\end{array}$ $\begin{array}{llllllll}0.421203 & 0.225169 & 0.578797 & 0.774831 & 2.783820 \mathrm{E}-12 & 9.976677 \mathrm{E}-07 & 2.688821 \mathrm{E}-08\end{array}$ $\begin{array}{llllllll}0.422894 & 0.225002 & 0.577106 & 0.774998 & 2.783264 \mathrm{E}-12 & 9.976748 \mathrm{E}-07 & 7.075852 \mathrm{E}-09\end{array}$ $\begin{array}{llllllll}0.425016 & 0.224795 & 0.574984 & 0.775205 & 2.819403 \mathrm{E}-12 & 9.976841 \mathrm{E}-07 & 4.387033 \mathrm{E}-08\end{array}$ $\begin{array}{lllllllll}0.429270 & 0.224377 & 0.570730 & 0.775623 & 2.732493 \mathrm{E}-12 & 9.977074 \mathrm{E}-07 & 1.556693 \mathrm{E}-08\end{array}$ $\begin{array}{llllllll}0.435641 & 0.223766 & 0.564359 & 0.776234 & 2.7324931 \mathrm{E}-12 & 9.9770732 \mathrm{E}-07 & 4.676093 \mathrm{E}-08\end{array}$ $\begin{array}{lllllll}0.478400 & 0.219459 & 0.521600 & 0.780541 & 2.889050 \mathrm{E}-12 & 9.979560 \mathrm{E}-07 & 3.396520 \mathrm{E}-08\end{array}$ $\begin{array}{llllllll}0.465735 & 0.213439 & 0.534265 & 0.786561 & 2.910560 \mathrm{E}-12 & 9.983185 \mathrm{E}-07 & 3.396634 \mathrm{E}-08\end{array}$

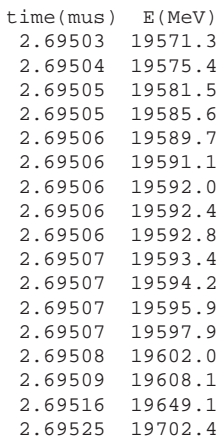


$\gamma G=48-\nu_{z}(19.59585 \mathrm{GeV})-\epsilon_{z} / \pi=210^{-6}$

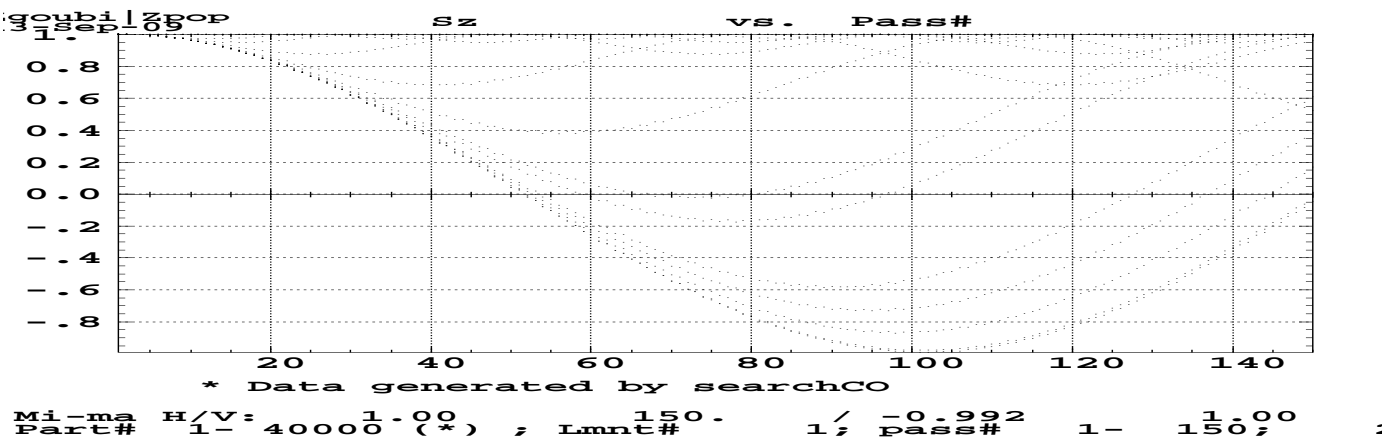

Figure 146: $S_{z}$ versus turn number for various distances to the resonance.
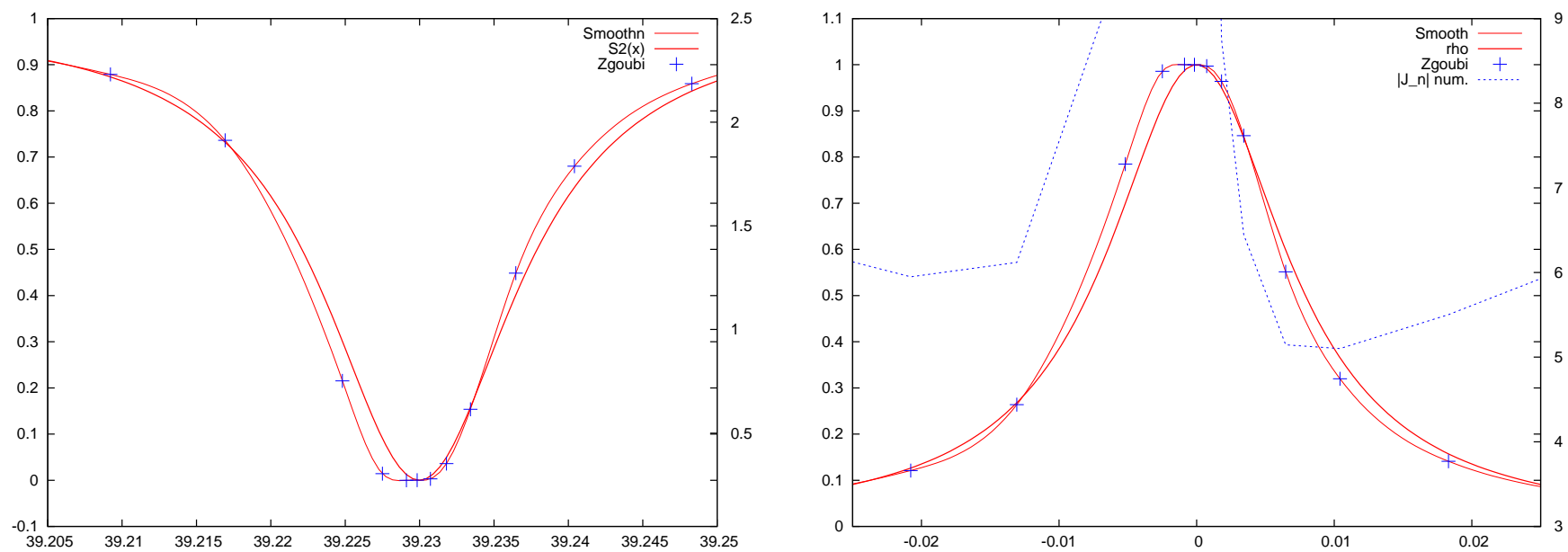

Figure 147: Matching $S_{z}^{2}(\Delta)$ (left, Eq. 4) and $\rho^{2}(\Delta)$ (right, Eq. 5) The right plot also shows $\left|J_{n}\right|^{2}$ from numerical data (with expectable lack of accuracy in the region $\Delta \rightarrow 0$ ).
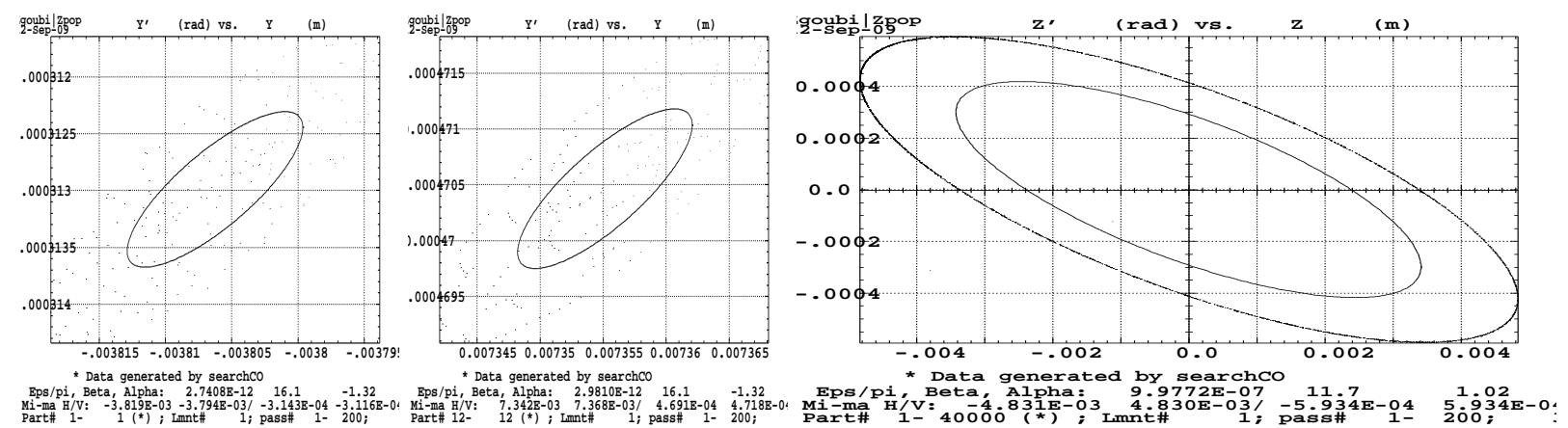

Figure 148: Left : $\mathrm{x}-\mathrm{x}^{\prime}$ of particles at min. and $\max . p / p_{0}$. 


\subsection{3 $\gamma G=36+\nu_{z}(\mathbf{2 2 . 4 8 8 3 2} \mathbf{~ G e V})$}

\section{Strengths}

Resonance strengths and vertical tunes are derived from the numerical simulation results by matching of numerical $\bar{S}_{z}(\gamma G)$ using Eq. 4, page 10.

This yields the results in Tab. 8 .

Table 8: Dependence of resonance strength squared $\left(\left|J_{n}\right|^{2}\right)$ on vertical invariant $\left(\epsilon_{z}\right) . \quad \nu_{z}$ compares fairly well with Fourier analysis of particle motion on $\epsilon_{z}$ invariant.

$\begin{array}{ccccc}\epsilon_{z} / \pi & \nu_{z} & \nu_{z} & \left|J_{n}\right|^{2} & \left|J_{n}\right|^{2} / \epsilon_{z} / \pi \\ \left(10^{-6} \mathrm{~m} . \mathrm{rad}\right) & \text { (Fourier) } & \text { (fit) } & \left(10^{-5}\right) & \\ 0.0017 & .763 & 8.7631 & 0.80592 & 4700 \\ 0.17 & & 8.7639 & 94.5166 & 5559\end{array}$


$\gamma G=36+\nu_{z}(22.48832 \mathrm{GeV})-\epsilon_{z} / \pi=0.001710^{-6}$

\section{Tracking data}

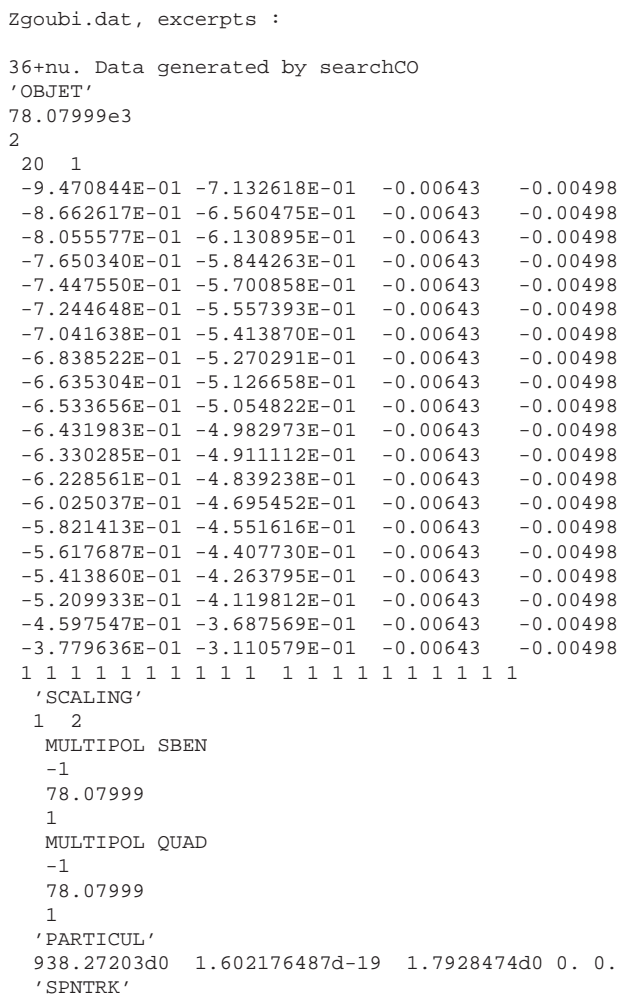

$0.0 \mathrm{E}+00 \quad 9.98500000 \mathrm{E}-01^{\prime} \mathrm{p} \mathrm{p}^{\prime}$

$22453.23 \mathrm{MeV}$ $0.0 \mathrm{E}+00 \quad 9.99200000 \mathrm{e}-01, \mathrm{~s}^{\prime}$, $0.0 \mathrm{E}+00 \mathrm{~g} 2469.61 \mathrm{MeV}$ $0.0 \mathrm{E}+00 \quad 9.99400000 \mathrm{E}-01 \mathrm{C}^{\prime} \mathrm{p}$ ' $22474.28 \mathrm{MeV}$ $0.0 \mathrm{E}+00 \quad 9.99500000 \mathrm{E}-011^{\prime} \mathrm{p} \mathrm{p}^{\prime} \quad 22476.62 \mathrm{MeV}$ $0.0 \mathrm{E}+00 \quad 9.99600000 \mathrm{E}-01^{\prime} \mathrm{p}^{\prime} \quad 22478.96 \mathrm{MeV}$ $0.0 \mathrm{E}+00 \quad 9.99700000 \mathrm{E}-01^{\prime} \mathrm{p}^{\prime} \quad 22481.30 \mathrm{MeV}$ $0.0 \mathrm{E}+00 \quad 9.99800000 \mathrm{E}-011^{\prime} \mathrm{p}$ ' $22483.64 \mathrm{MeV}$ $0.0 \mathrm{E}+00 \quad 9.99900000 \mathrm{E}-011^{\prime} \mathrm{p}$ ' $22485.98 \mathrm{MeV}$ $0.0 \mathrm{E}+00 \quad 9.99950000 \mathrm{E}-01$ ' $^{\prime} \mathrm{p}$ ' $22487.15 \mathrm{MeV}$ $0.0 \mathrm{E}+00 \quad 1.00000000 \mathrm{E}+00^{\prime} \mathrm{p}^{\prime} \quad 22488.32 \mathrm{MeV}$

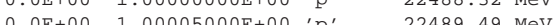
$0.0 \mathrm{E}+001.00005000 \mathrm{E}+00$ " $\mathrm{p}$ ", $22489.49 \mathrm{MeV}$ $0.0 \mathrm{E}+001.00010000 \mathrm{E}+00 \mathrm{c}$ "p" $22490.66 \mathrm{MeV}$ $0.0 \mathrm{E}+00 \quad 1.00020000 \mathrm{E}+00^{\prime} \mathrm{p}^{\prime} \quad 22493.00 \mathrm{MeV}$ $0.0 \mathrm{E}+00 \quad 1.00030000 \mathrm{E}+00^{\prime} \mathrm{p}^{\prime} \quad 22495.33 \mathrm{MeV}$ $0.0 \mathrm{E}+00 \quad 1.00040000 \mathrm{E}+00^{\prime} \mathrm{p}$ ' $22497.67 \mathrm{MeV}$ $0.0 \mathrm{E}+00 \quad 1.00050000 \mathrm{E}+00^{\prime} \mathrm{p}^{\prime} \quad 22500.01 \mathrm{MeV}$ $0.0 \mathrm{E}+00 \quad 1.00060000 \mathrm{E}+00^{\prime} \mathrm{p}^{\prime} \quad 22502.35 \mathrm{MeV}$ $0.0 \mathrm{E}+00 \quad 1.00090000 \mathrm{E}+00^{\prime} \mathrm{p}^{\prime} \quad 22509.37 \mathrm{MeV}$ $0.0 \mathrm{E}+00 \quad 1.00130000 \mathrm{E}+00^{\prime} \mathrm{p}^{\prime} \quad 22518.72 \mathrm{MeV}$

Checking computation results : for the 20 particles above, from 200 turns analysis, their $\mathrm{H}$ closed orbits, fractional tunes (Fourier analysis) and complements to $1, \mathrm{H}$ and $\mathrm{V}$ invariant values, energy :

\begin{tabular}{|c|c|c|c|c|c|c|c|c|c|c|}
\hline $\mathrm{xCO}$ & $x^{\prime} \mathrm{CO}$ & $\mathrm{XNU}$ & $\mathrm{zNU}$ & NU & $\mathrm{ZNU}$ & \multicolumn{3}{|c|}{ epsilon/pi_X,z, I } & time (mus) & $\mathrm{E}(\mathrm{MeV})$ \\
\hline $9.470872 \mathrm{E}-03$ & $-7.132596 \mathrm{E}-04$ & 0.256813 & 0.238834 & 0.743187 & 0.761166 & $1.324362 \mathrm{E}-16$ & $8.428865 \mathrm{E}-10$ & $5.941624 \mathrm{E}-08$ & 2.69412 & 22453.2 \\
\hline $663059 \mathrm{E}-03$ & $-6.560778 \mathrm{E}-04$ & .265148 & 238243 & 734852 & 761757 & $1.321423 \mathrm{E}-15$ & $8.425258 \mathrm{E}-10$ & $1.188331 \mathrm{E}-07$ & 2.69413 & 22462.6 \\
\hline$-8.055589 \mathrm{E}-03$ & $-6.130968 \mathrm{E}-04$ & 0.271368 & 0.237784 & 0.728632 & 0.762216 & $2.727146 \mathrm{E}-16$ & $8.422477 \mathrm{E}-10$ & $8.318346 \mathrm{E}-08$ & .69414 & \\
\hline$-7.650366 \mathrm{E}-03$ & $-5.844278 \mathrm{E}-04$ & .275550 & .237473 & 0.724450 & .762527 & $6.013444 \mathrm{E}-17$ & $8.420647 \mathrm{E}-10$ & $1.222291 \mathrm{E}-07$ & 69415 & 2474.3 \\
\hline $447593 \mathrm{E}-03$ & $-5.700916 \mathrm{E}-04$ & 277636 & 315 & 2364 & 685 & $300 \mathrm{E}-17$ & $8.419734 \mathrm{E}-10$ & $679 E-08$ & & 76.6 \\
\hline$-7.244645 \mathrm{E}-03$ & $-5.557404 \mathrm{E}-04$ & .279712 & .237178 & 20288 & 762822 & & -10 & 8. & & 9.0 \\
\hline$-7.041638 \mathrm{E}-03$ & $-5.413877 \mathrm{E}-04$ & .281785 & 0.237017 & 0.718215 & 0.762983 & -18 & -10 & $E-08$ & & 1.3 \\
\hline-6.838523 & $-5.270286 \mathrm{E}-04$ & 283866 & .2 & & & -18 & -10 & & & \\
\hline-6.635325 & $-5.126677 \mathrm{E}-04$ & .285951 & 0.236703 & 0.714049 & 763297 & $E-18$ & $8.416126 \mathrm{E}-10$ & 6.960 & 17 & \\
\hline$-6.533659 \mathrm{E}-03$ & $-5.054828 \mathrm{E}-04$ & 0.287002 & 0.236629 & 0.712998 & 0.763371 & & $669 \mathrm{E}-10$ & $E-08$ & & \\
\hline$-6.431985 \mathrm{E}-03$ & $-4.982972 \mathrm{E}-04$ & 288054 & 46 & 6 & 54 & -18 & -10 & -07 & & 3.3 \\
\hline$-6.330300 \mathrm{E}-03$ & $-4.911119 \mathrm{E}-04$ & .289100 & .23 & 00 & 548 & -18 & -10 & $9 \mathrm{E}-08$ & 2.6 & 9.5 \\
\hline$-6.228580 \mathrm{E}-03$ & $-4.839258 \mathrm{E}-04$ & 0.290140 & 0.236369 & 0.709860 & 0.763631 & -18 & $E-10$ & $5 \mathrm{E}-08$ & 17 & 0.7 \\
\hline$-6-025030 F-03$ & $-4.695446 \mathrm{E}-04$ & 92219 & 25 & & & -18 & -10 & $E-08$ & & \\
\hline$-5.821433 \mathrm{E}-03$ & $-4.551624 \mathrm{E}-04$ & 294294 & 72 & 06 & 28 & $E-18$ & -10 & $7 \mathrm{E}-07$ & & 22 \\
\hline$-5.617702 \mathrm{E}-03$ & $-4.407750 \mathrm{E}-04$ & 0.296376 & 0.235887 & 0.703624 & 0.764113 & $5.242630 \mathrm{E}-18$ & $8.411499 \mathrm{E}-10$ & $352 \mathrm{E}-08$ & 2.69418 & 22497.7 \\
\hline $5 \quad 1138595=03$ & $-4.263791 \mathrm{E}-04$ & & & & & & -10 & $E-08$ & & \\
\hline$-5.209948 \mathrm{E}-03$ & $20 \mathrm{E}-04$ & 71 & 37 & 9 & 3 & -18 & -10 & -07 & & .4 \\
\hline$-4.597559 \mathrm{E}-03$ & $-3.687581 \mathrm{E}-04$ & 0.306818 & 0.235096 & 0.693182 & 0.764904 & $6.153343 \mathrm{E}-18$ & $8.406965 \mathrm{E}-10$ & $1.018594 \mathrm{E}-08$ & 2.69420 & 22509.4 \\
\hline$-3.779643 \mathrm{E}-03$ & $-3.110580 \mathrm{E}-04$ & 0.315191 & 0.234410 & 0.684809 & 0.765590 & $5.334159 \mathrm{E}-18$ & $8.403249 \mathrm{E}-10$ & $8.318563 \mathrm{E}-08$ & 2.69421 & 22518.7 \\
\hline
\end{tabular}


$\gamma G=36+\nu_{z}(22.48832 \mathrm{GeV})-\epsilon_{z} / \pi=0.001710^{-6}$

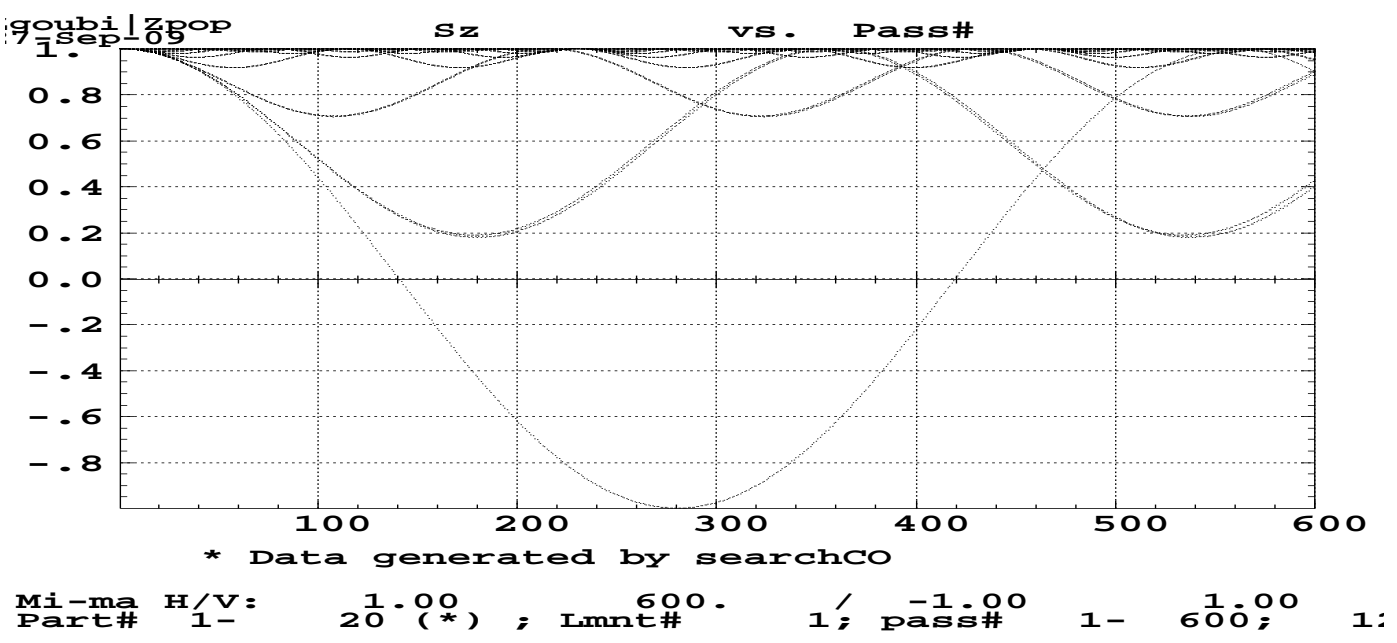

Figure 149: $S_{z}$ versus turn number for various distances to the resonance.
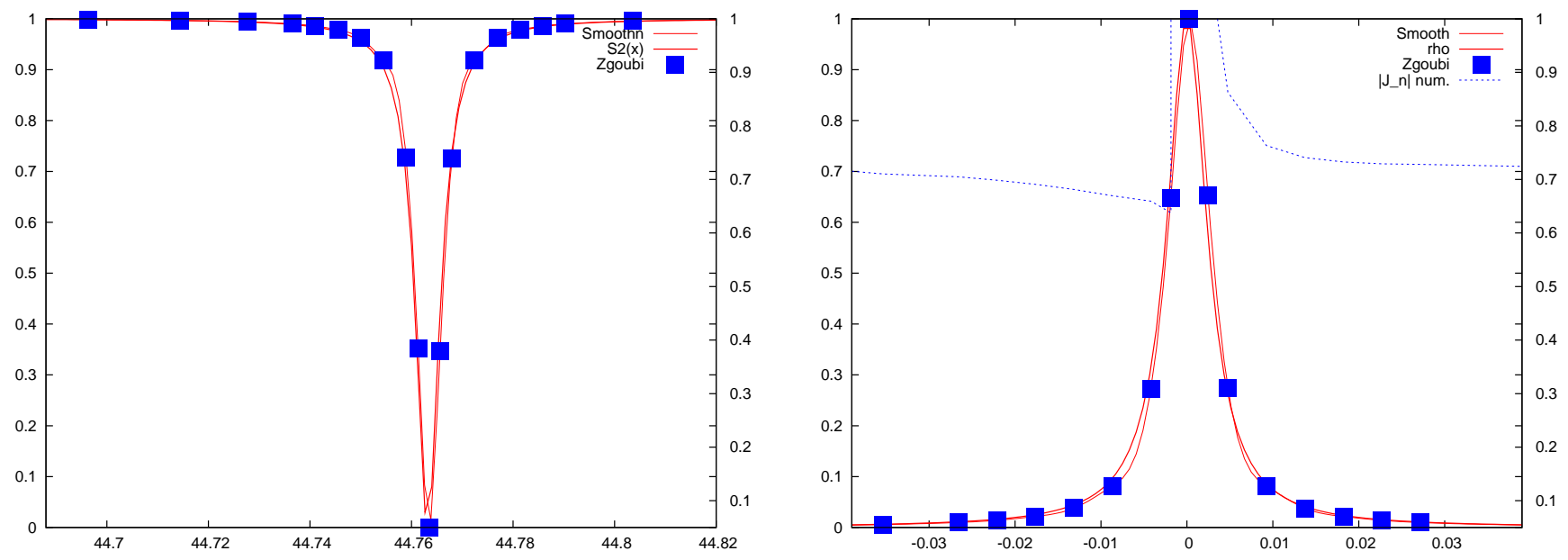

Figure 150: Matching $S_{z}^{2}(\Delta)$ (left, Eq. 4) and $\rho^{2}(\Delta)$ (right, Eq. 5) The right plot also shows $\left|J_{n}\right|^{2}$ from numerical data (with expectable lack of accuracy in the region $\Delta \rightarrow 0$ ).
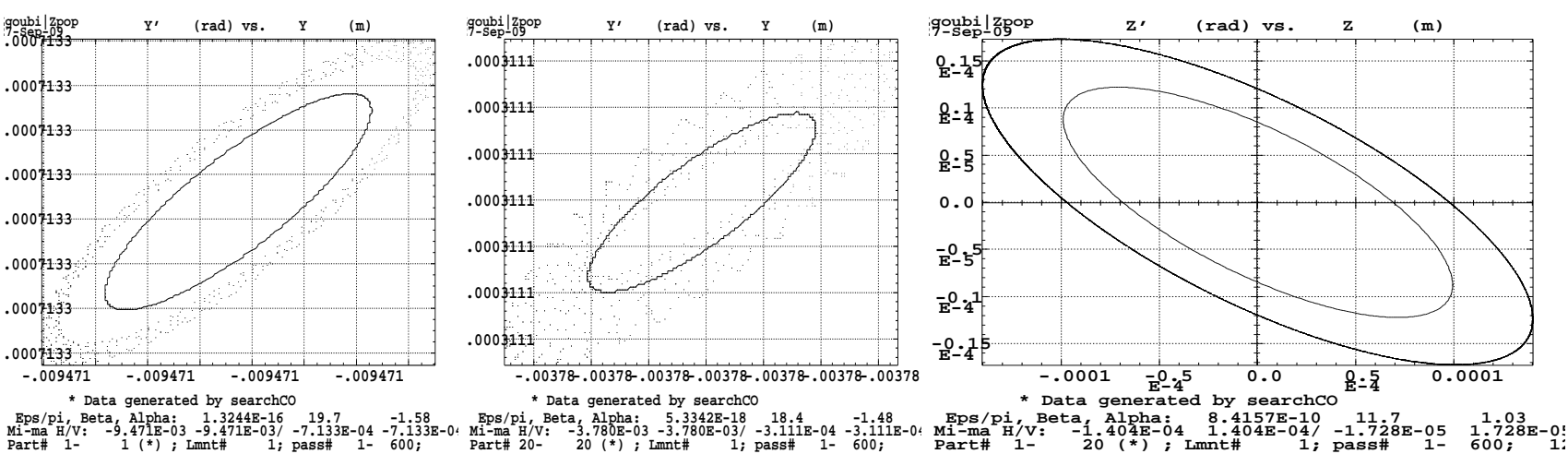

Figure 151: Left : $\mathrm{x}-\mathrm{x}$ ' of particles at min. and max. $p / p_{0}$. Right : z-z' (all particles superimposed). 
$\gamma G=36+\nu_{z}(22.48832 \mathrm{GeV})-\epsilon_{z} / \pi=0.1710^{-6}$

\section{Tracking data}

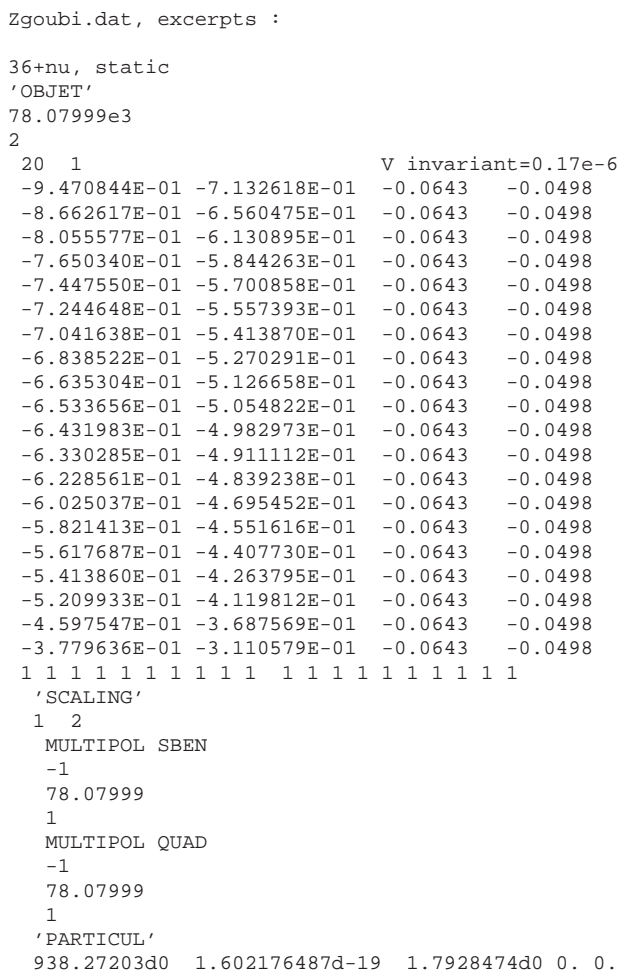

Checking computation results : for the 20 particles above, from 200 turns analysis, their $\mathrm{H}$ closed orbits, fractional tunes (Fourier analysis) and complements to $1, \mathrm{H}$ and $\mathrm{V}$ invariant values, energy :

\begin{tabular}{|c|c|c|c|c|c|c|c|c|c|c|}
\hline $\mathrm{xCO}$ & $\mathrm{X}^{\prime} \mathrm{CO}$ & $\mathrm{XNU}$ & $\mathrm{zNU}$ & $-\mathrm{XNU}$ & $1-\mathrm{ZNU}$ & epsilor & pi_x, z, I & & time (mus) & $\mathrm{E}(\mathrm{MeV})$ \\
\hline $471191 \mathrm{E}-03$ & $-7.132492 \mathrm{E}-04$ & 256831 & .238846 & 0.7431 & 0.7611 & $320 \mathrm{E}-14$ & $8.428749 \mathrm{E}-08$ & $38 \mathrm{E}-$ & 2.69412 & 22453.2 \\
\hline$-8.665436 \mathrm{E}-03$ & $-6.562084 E-04$ & 0.265155 & 0.238248 & 0.734845 & 0.761752 & $.102949 \mathrm{E}-14$ & $8.424973 \mathrm{E}-08$ & $1.188318 \mathrm{E}-08$ & 59413 & 462.6 \\
\hline $6784 \mathrm{E}-03$ & $-6.132896 \mathrm{E}-04$ & 71387 & 19 & 28613 & & $524 \mathrm{E}-14$ & $212 \mathrm{E}-08$ & & & \\
\hline $649985 \mathrm{E}-03$ & $-5.843489 E-04$ & 275548 & .237463 & .724452 & 0.762537 & $96437 \mathrm{E}-14$ & $8.420384 \mathrm{E}-08$ & $51 \mathrm{E}-08$ & 15 & 1.3 \\
\hline$-7.449236 \mathrm{E}-03$ & $-5.701867 \mathrm{E}-04$ & 0.277640 & 0.237307 & .722360 & 0.762693 & $424775 \mathrm{E}-14$ & $8.419471 \mathrm{E}-08$ & $36 \mathrm{E}-09$ & & 6.6 \\
\hline$-7.246618 \mathrm{E}-03$ & $-5.559006 \mathrm{E}-04$ & .279730 & .237154 & 20270 & 346 & $044 \mathrm{E}-14$ & $59 \mathrm{E}-08$ & 88 & & 9.0 \\
\hline-03 & -5.41 & & 998 & 3199 & & -14 & & & & \\
\hline$-6.838782 \mathrm{E}-03$ & $-5.269547 \mathrm{E}-04$ & 0.283885 & .236844 & & 0.7 & $327 E-14$ & $E-08$ & & & 3.6 \\
\hline-03 & -5.128 & 44 & 93 & & & -14 & $\mathrm{E}-08$ & & & 5.0 \\
\hline$-6.534031 \mathrm{E}-03$ & $-5.055584 \mathrm{E}-04$ & .287019 & 6616 & 81 & & -14 & -08 & & & 7.1 \\
\hline$-6.432217 \mathrm{E}-03$ & $-4.982846 \mathrm{E}-04$ & 0.288055 & 0.236539 & 0.711945 & 0.763461 & $5.505559 \mathrm{E}-14$ & $8.414952 \mathrm{E}-08$ & EE-09 & 17 & 22488.3 \\
\hline-03 & -4 . & & 0 & & & -14 & -08 & & & \\
\hline $6 \mathrm{E}-03$ & $E-04$ & & & & & 14 & 8 & & & .7 \\
\hline$-6.024385 E-03$ & $-4.694925 E-04$ & 0.292236 & 0.236227 & 0.707764 & 0.763773 & $5.515216 \mathrm{E}-14$ & $3197 \mathrm{E}-08$ & 1.18 & 18 & 22493.0 \\
\hline & & & & & & -14 & -08 & & & .3 \\
\hline $26 \mathrm{E}-03$ & -4.409 & & & & & -14 & 08 & & & \\
\hline $413718 \mathrm{E}-03$ & $-4.263393 \mathrm{E}-04$ & 60 & .235742 & 10 & 0.7 & $948 \mathrm{E}-14$ & $E-08$ & $E-09$ & & 0.0 \\
\hline$-5.211530 \mathrm{E}-03$ & $-4.120730 \mathrm{E}-04$ & 0.300571 & 0.235577 & 0.699429 & 0.764423 & $5.505439 \mathrm{E}-14$ & $8.409755 \mathrm{E}-08$ & $84 \mathrm{E}-$ & 2 . & 22502.4 \\
\hline & -3 . & & & & & -14 & & & & \\
\hline $30573 E-03$ & $-3.110925 E-04$ & 315188 & 234408 & 0.684812 & .765592 & $5.530870 \mathrm{E}-14$ & $8.403336 \mathrm{E}-08$ & $1.697647 \mathrm{E}-08$ & 2.69421 & 22518.7 \\
\hline
\end{tabular}


$\gamma G=36+\nu_{z}(22.48832 \mathrm{GeV})-\epsilon_{z} / \pi=0.1710^{-6}$

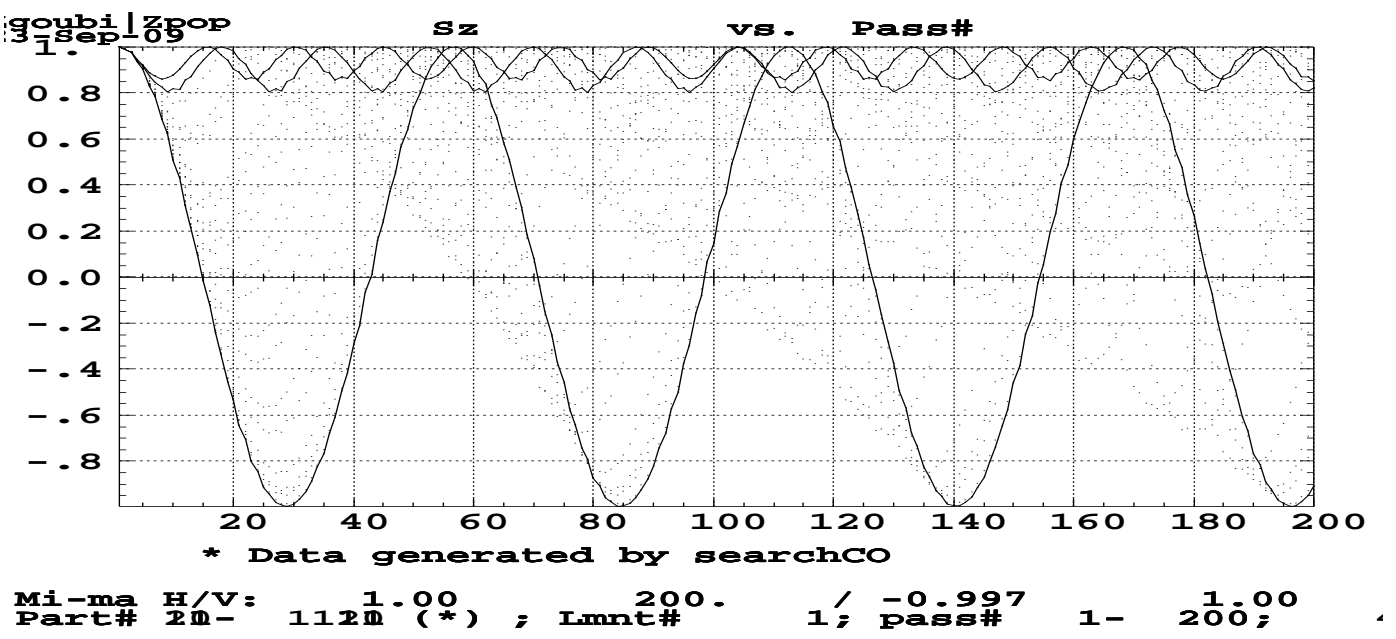

Figure 152: $S_{z}$ versus turn number for various distances to the resonance.
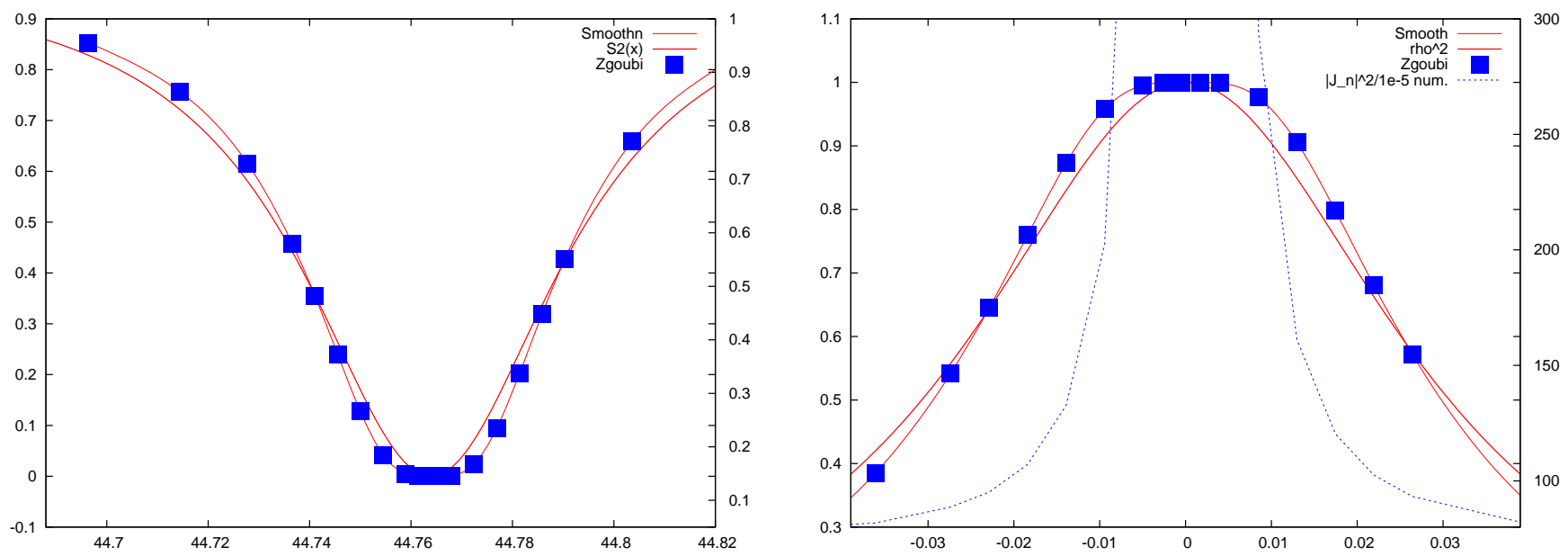

Figure 153: Matching $S_{z}^{2}(\Delta)$ (left, Eq. 4) and $\rho^{2}(\Delta)$ (right, Eq. 5) The right plot also shows $\left|J_{n}\right|^{2}$ from numerical data (with expectable lack of accuracy in the region $\Delta \rightarrow 0$ ).
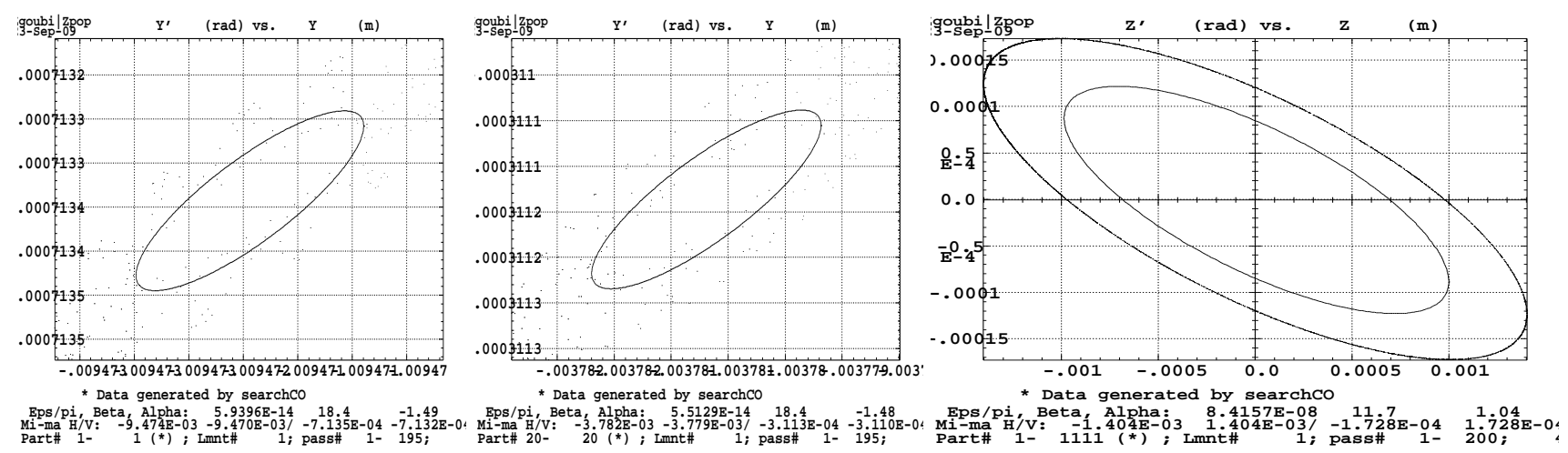

Figure 154: Left : $\mathrm{x}-\mathrm{x}$ ' of particles at min. and max. $p / p_{0}$. Right : z-z' (all particles superimposed). 


\section{Fresnel integrals approximation of weak resonances}

The goal of this section is to check zgoubi against the weak resonance approximation of resonance crossing dynamics, see Sec. 2.3.4.

Matching the tracking results by the Fresnel integral approximation would yield the crossing speed $\alpha$ and the resonance strength $\left|J_{n}\right|$. In the figures below crossing velocity is taken to be the same as used earlier, $\alpha=$ $4.409635610^{-5}$, and resonance strength is taken from Tabs. 2, 3, Froissard-Stora crossing conditions, whereas $\nu_{z}=8.76346$ from Zgoubi, as earlier.

- $\gamma G=\nu_{z}(3.648013 \mathrm{GeV})-\epsilon_{z} / \pi=0.00210^{-6}$

$\pi\left|J_{n}\right|^{2} / \alpha \approx 0.014$, higher order terms have negligible effect.

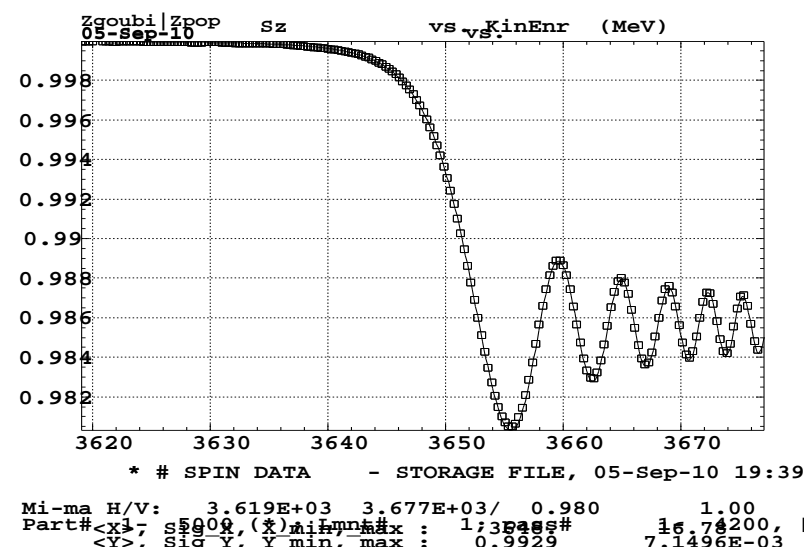

Figure 155: Superimposition of Fresnel integral model (squares) and zgoubi tracking (solid line), $\epsilon_{z} / \pi=0.00210^{-6}$.

- $\gamma G=\nu_{z}(3.648013 \mathrm{GeV})-\epsilon_{z} / \pi=0.0110^{-6}$

$\pi\left|J_{n}\right|^{2} / \alpha \approx 0.071$, , higher order terms have sensible effect.

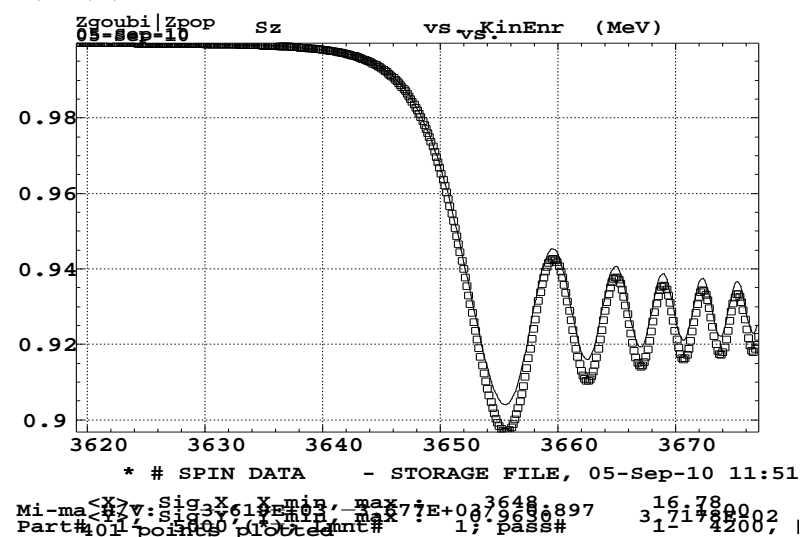

Figure 156: Superimposition of Fresnel integral model (squares) and zgoubi tracking (solid line) in a case of greater effect compared to the previous Fig. $155: \epsilon_{z} / \pi=$ $0.0110^{-6}$. The truncation of Froissard-Stora formula leads to an overestimate of $p_{f} / p_{i}$ (Eq. 7).

$$
\begin{gathered}
\left(\frac{p(\theta)}{p_{i}}\right)^{2}=1-\frac{\pi}{\alpha}\left|J_{n}\right|^{2}\left[\left(0.5-C\left(-\theta \sqrt{\frac{a}{\pi}}\right)\right)^{2}+\right. \\
\left.\left(0.5-S\left(-\theta \sqrt{\frac{a}{\pi}}\right)\right)^{2}\right] \\
\bullet \theta>0: \\
\left(\frac{p(\theta)}{p_{i}}\right)^{2}=1-\frac{\pi}{\alpha}\left|J_{n}\right|^{2}\left[\left(0.5+C\left(\theta \sqrt{\frac{a}{\pi}}\right)\right)^{2}+\right. \\
\left.\left(0.5+S\left(\theta \sqrt{\frac{a}{\pi}}\right)\right)^{2}\right] \\
C(x)=\int_{0}^{x} \cos \left(\frac{\pi}{2} t^{2}\right) d t, S(x)=\int_{0}^{x} \sin \left(\frac{\pi}{2} t^{2}\right) d t
\end{gathered}
$$

$2 \exp \left(-A^{2}\right)-1 \approx 1-A^{2}$ no longer holds 
- $\gamma G=36+\nu_{z}(22.4883 \mathrm{GeV})-\epsilon_{z} / \pi=0.000110^{-6}$

$\pi\left|J_{n}\right|^{2} / \alpha \approx 0.013$, higher order terms have negligible effect.

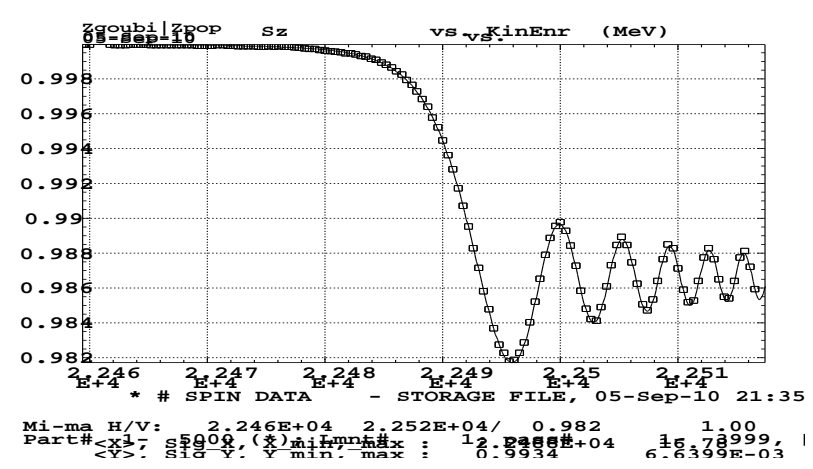

Figure 157: Superimposition of Fresnel integral model (squares) and zgoubi tracking (solid line) $\epsilon_{z} / \pi=0.000110^{-6}$.

- $\gamma G=9(3.7718 \mathrm{GeV})-z_{C O}=1.3 \mathrm{~mm}$

$\pi\left|J_{n}\right|^{2} / \alpha \approx 0.0059$, higher order terms have negligible effect.

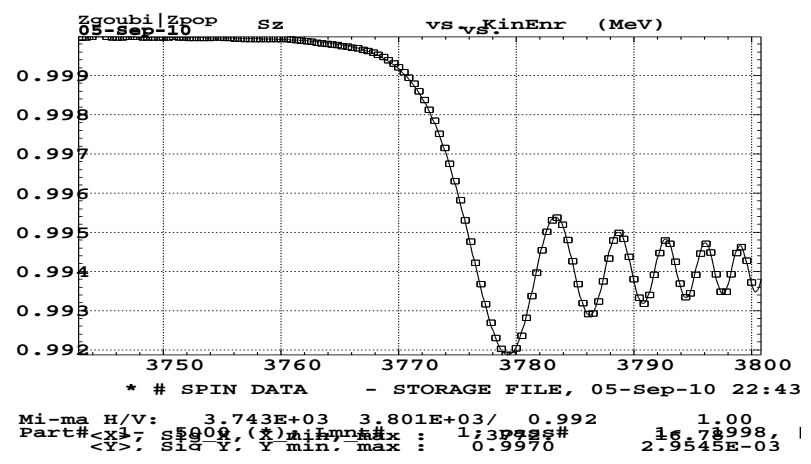

Figure 158: Superimposition of Fresnel integral model (squares) and zgoubi tracking (solid line).

- $\gamma G=45(22.61211 \mathrm{GeV})-z_{C O}=0.028 \mathrm{~mm}$

$\pi\left|J_{n}\right|^{2} / \alpha \approx 0.004$, higher order terms have negligible effect.

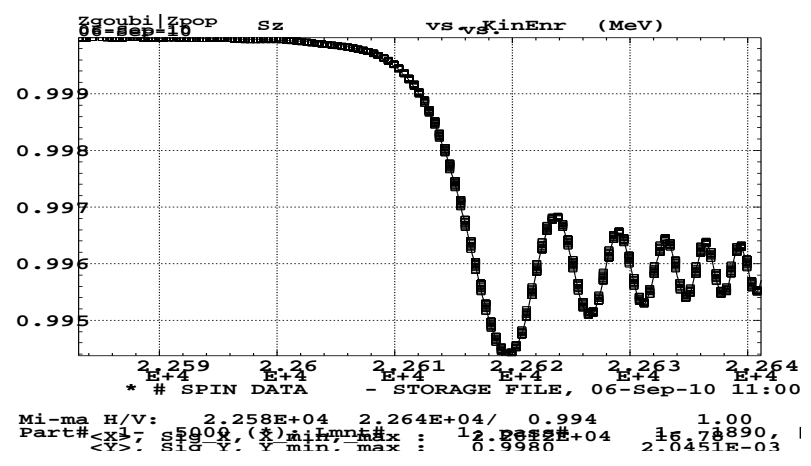

Figure 159: Superimposition of Fresnel integral model (squares) and zgoubi tracking (solid line). 


\section{$7 \quad \vec{n}_{0}$ spin vector, AGS bare lattice}

To perform this experiment, we consider a single particle at fixed energy, we sit at $T=3775.00 \mathrm{GeV}$ within the span of $\gamma G=9(\mathrm{~T}=3.7716 \mathrm{GeV})$. The resonance is excited by a $\hat{z} \approx 2.8 \mathrm{~mm}$ vertical closed orbit defect (same as earlier, Fig. 100 page 49).

The particle is launched on closed $\mathrm{H}$ and $\mathrm{V}$ orbit $\left(\epsilon_{x}=\epsilon_{z}=0\right.$, Fig. 160) with first $S_{z}=1$, the polarization vector then oscillates around the $\vec{n}_{0}$ vector, Fig. 161.

The FIT procedure allows finding $\vec{n}_{0}$, typical zgoubi.dat input in App. B.2.

Given the FIT results, the particle is then launched, again on closed $\mathrm{H}$ and V orbit, with now $\vec{S}=$ closed orbit $\vec{S}$. Fig. 162 shows that the polarization vector, as observed turn after turn at 'Begin SUPERA' (entrance to A1BF main dipole), is now stationary.
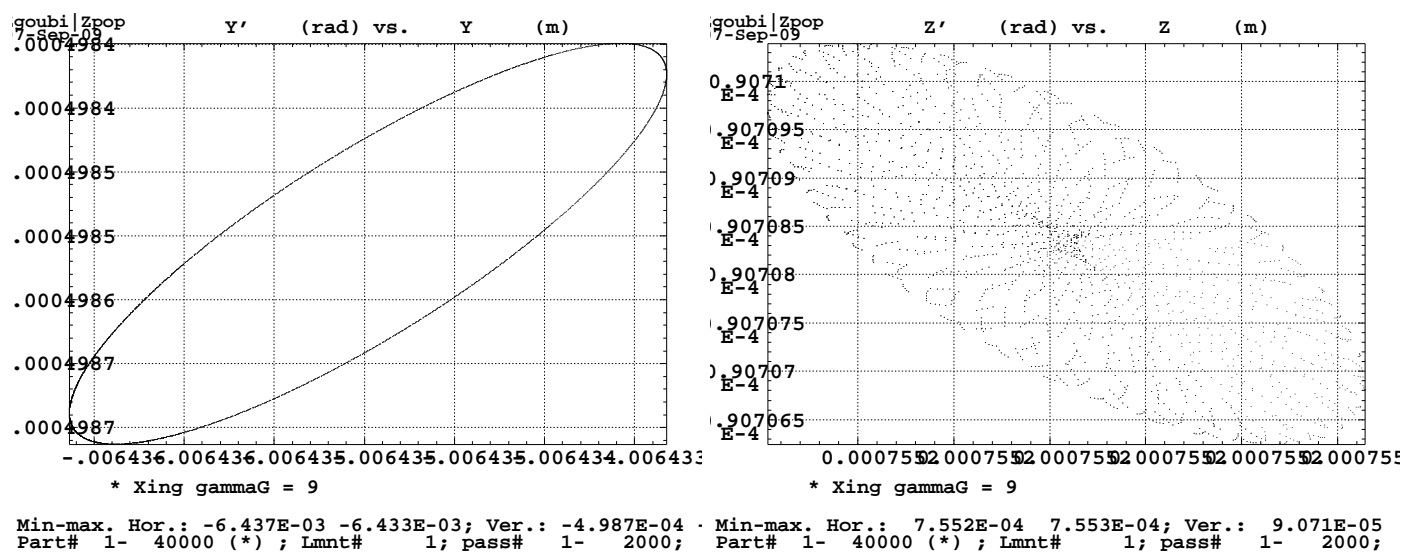

Figure 160: The particle sits on $\mathrm{H}$ and $\mathrm{V}$ closed orbits - observed at 'Begin SUPERA' (entrance to A1BF main dipole). Namely, the particle describes quasi zero $\epsilon_{x}$ and $\epsilon_{y}$ invariants, as shown here.

$$
\vec{S}_{\text {initial }}=\vec{S}_{z}
$$
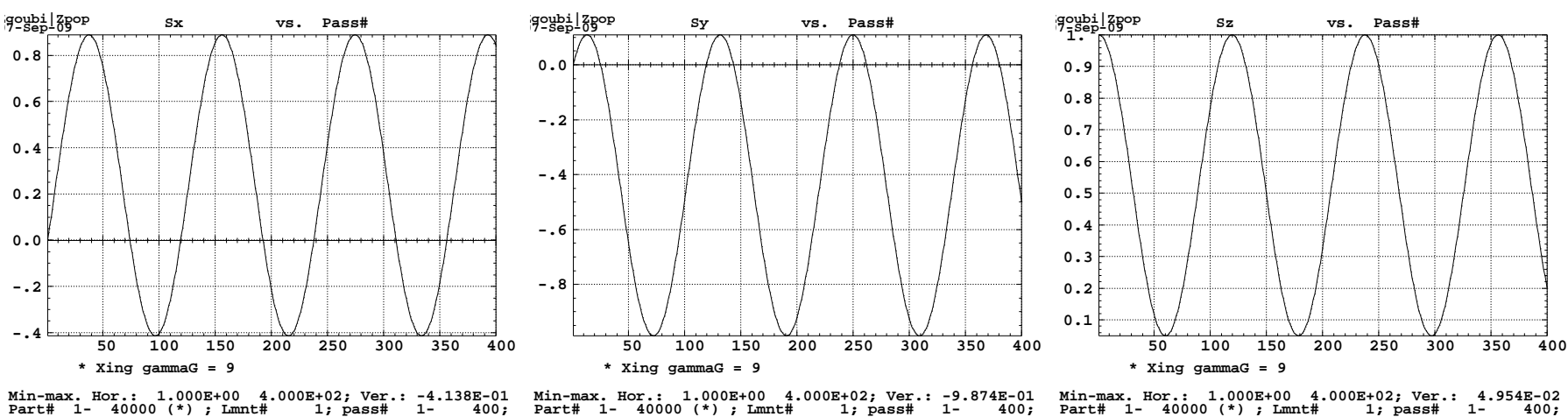

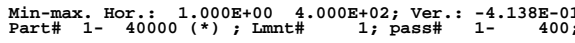

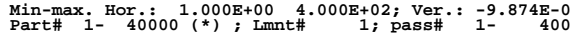

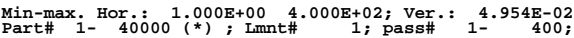

Figure 161: From top to bottom, $S_{x} S_{y} S_{z}$ versus turn number observed at 'Begin SUPERA'. Static case $(T=$ $3775.00 \mathrm{GeV}$ ), given $\vec{S}_{\text {initial }}=\vec{S}_{z}$. 


\section{$\vec{n}_{0}$ vector over a turn, $\vec{S}_{\text {initial }}=\vec{S}_{\text {final }} \equiv$ closed-orbit $\vec{S}$}

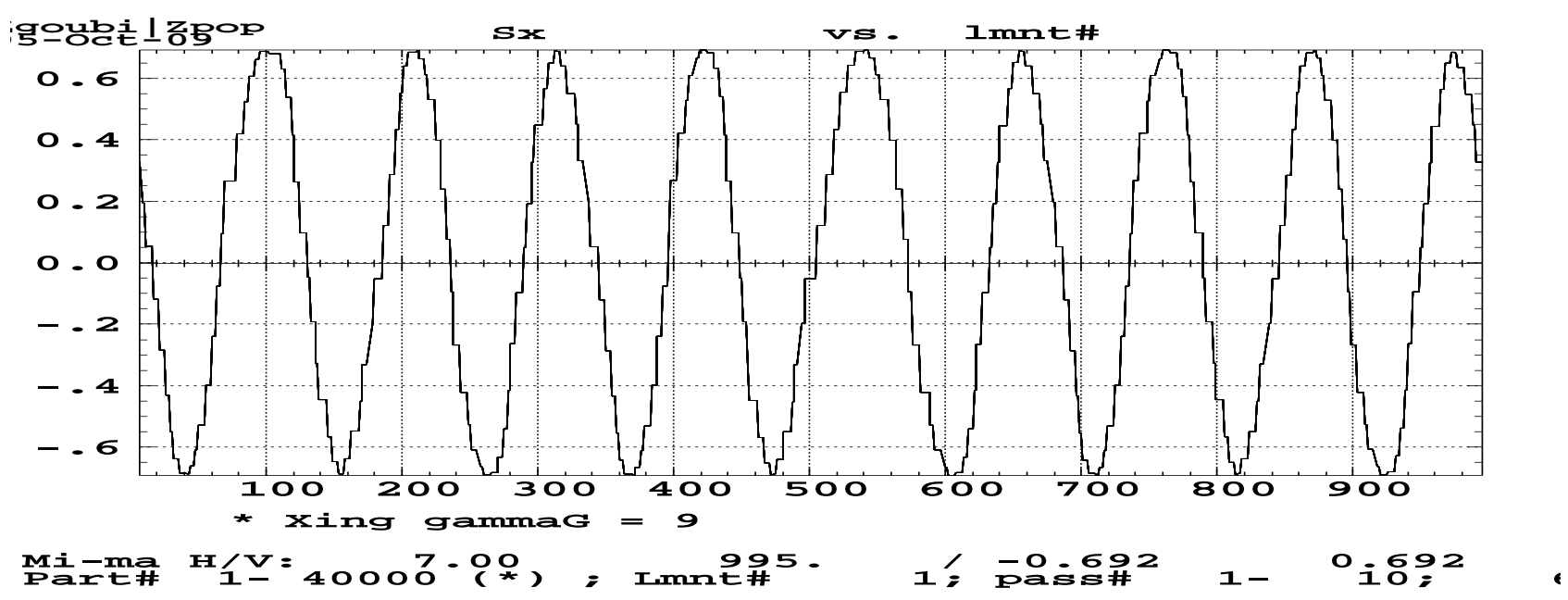

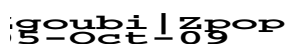

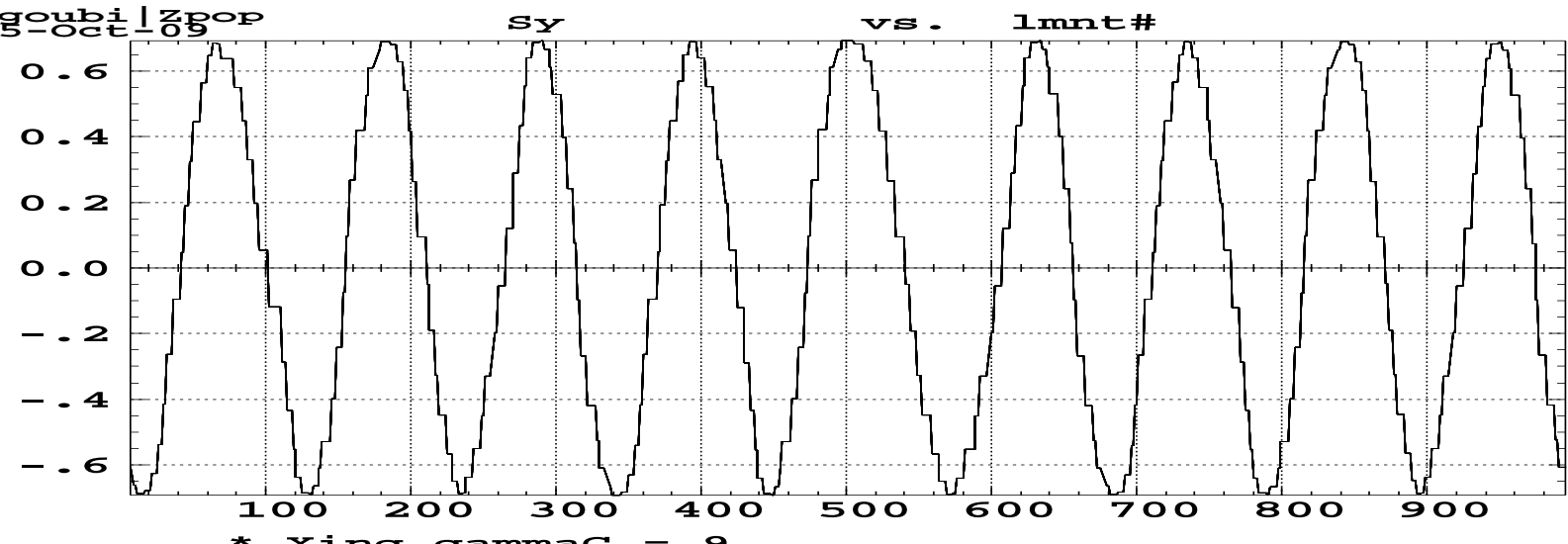

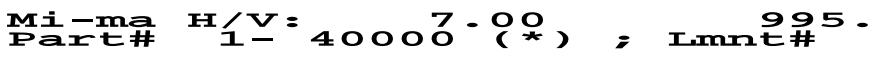

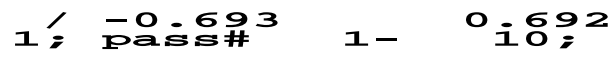

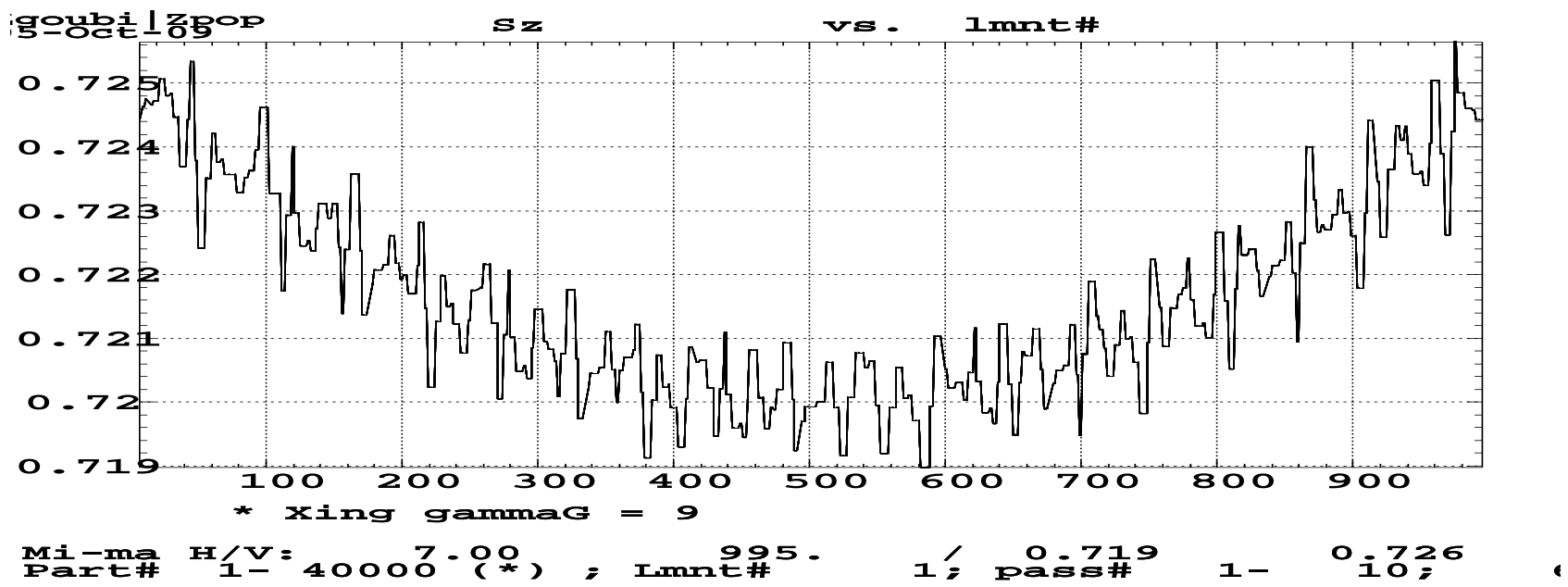

Figure 162: From top to bottom, $S_{x} S_{y} S_{z}$ along machine circumference versus pick-up number (about 990 P-Us over the $807 \mathrm{~m}$ circumference). Static case $(T=3775.00 \mathrm{GeV})$, given $\vec{S}_{\text {initial }} \equiv$ closed-orbit $\vec{S}$. 


\section{APPENDIX}

\section{A MAD files}

Excerpts of MAD files are reproduced, for reference.

\section{A.1 Command file}

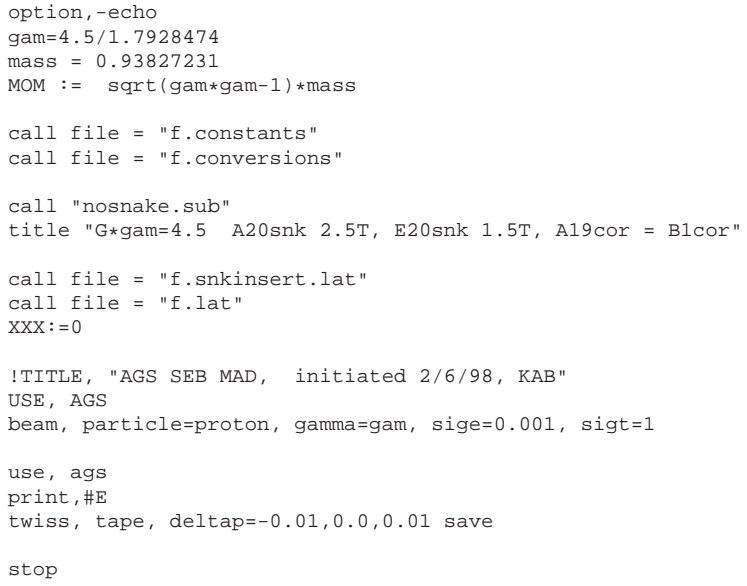

\section{A.2 "print" file}

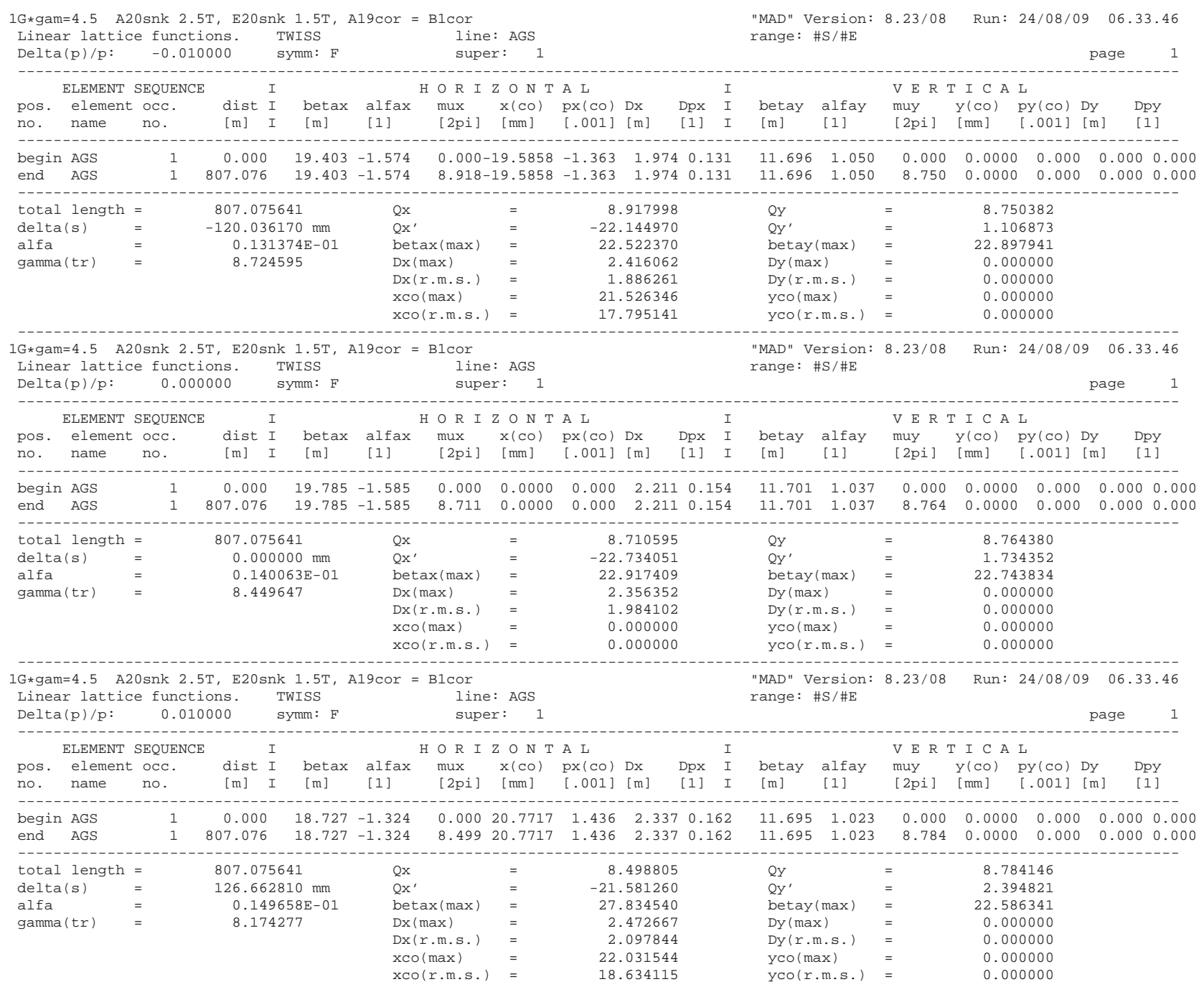




\section{B Zgoubi data file specimen}

\section{B.1 1-turn first order mapping calculation}

Typical zgoubi.dat file including appropriate commands for MATRIX calculation.

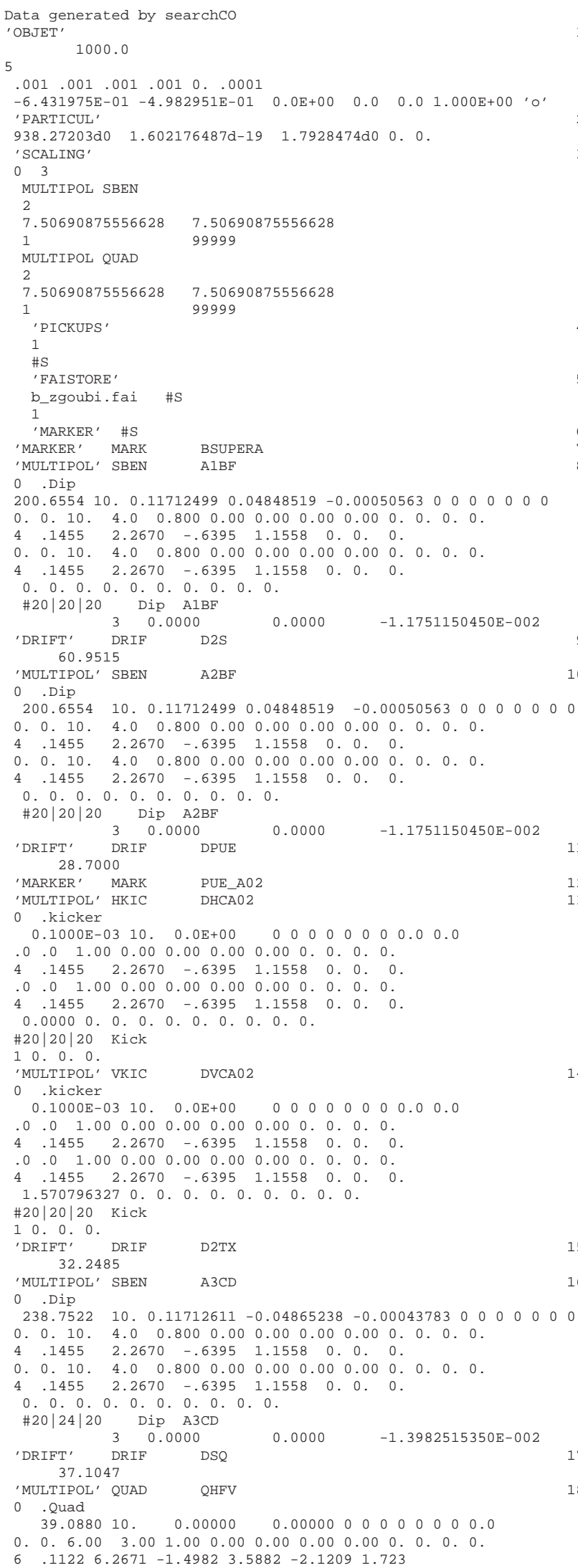




\section{B.2 Zgoubi files, $\vec{n}_{0}$ vector search using FIT}

\section{zgoubi.dat including FIT input :}

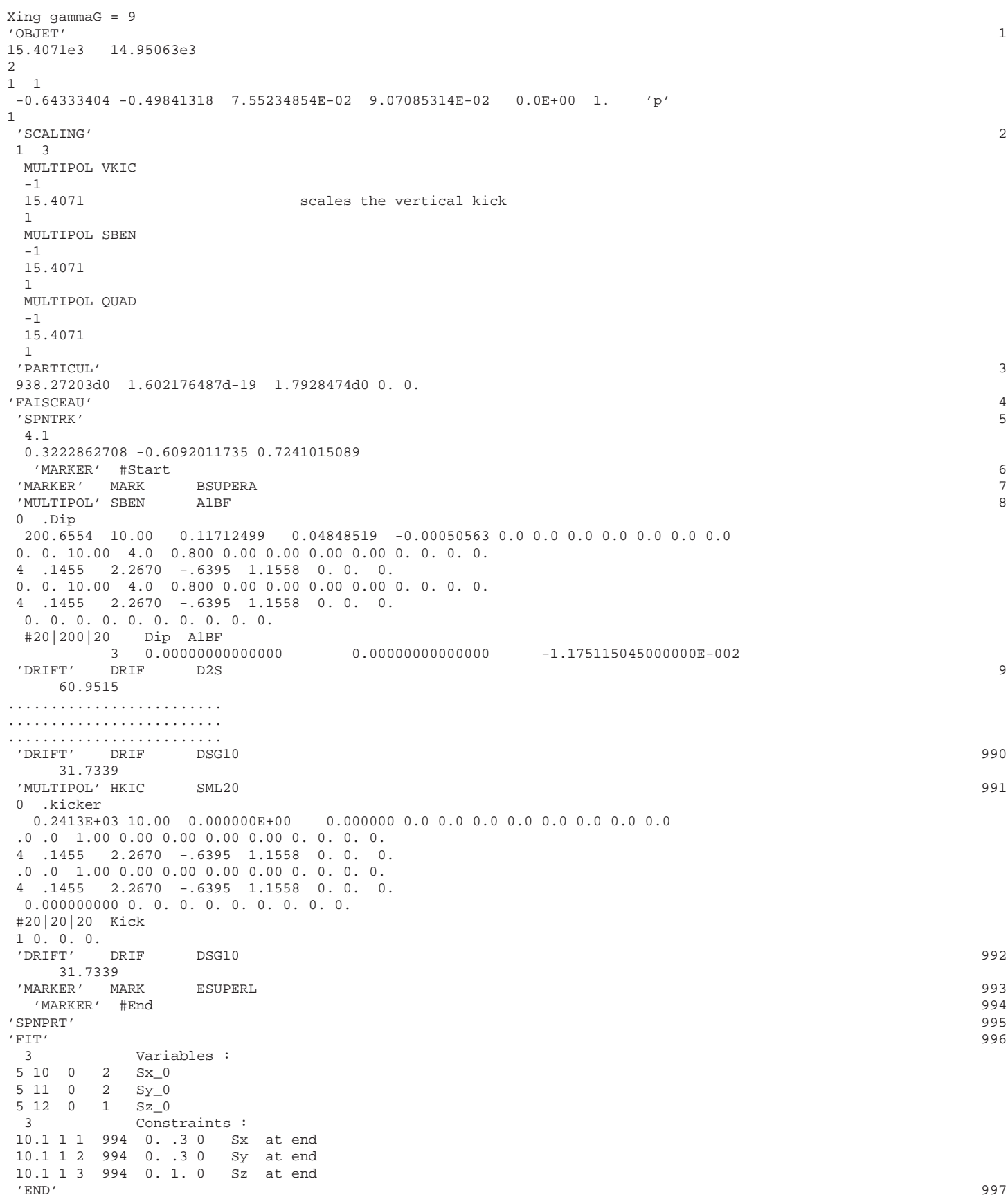

\section{zgoubi.res including FIT output :}

996 FIT

\begin{tabular}{|c|c|c|c|c|c|c|}
\hline variable \# & 1 & $\mathrm{IR}=$ & & 5, & & ok \\
\hline variable \# & 1 & IP $=$ & & 10, & & ok \\
\hline variable \# & 2 & $I R=$ & & 5, & & ok \\
\hline variable \# & 2 & IP $=$ & & 11, & & ok \\
\hline variable \# & 3 & $\mathrm{IR}=$ & & 5, & & ok \\
\hline variable \# & 3 & IP $=$ & & 12, & & ok \\
\hline constraint \# & 1 & IR $=$ & $=$ & 994 & 4 & \\
\hline constraint \# & 2 & $\mathrm{IR}=$ & $=$ & 994 & 4 & \\
\hline constraint \# & 3 & $\mathrm{IR}=$ & $=$ & 994 & 4 & \\
\hline
\end{tabular}

\begin{tabular}{|c|c|c|c|c|c|c|c|c|}
\hline \multicolumn{2}{|c|}{ STATUS OF } & \multicolumn{3}{|c|}{ (Iteration \# 0} & & & \multirow{2}{*}{\multicolumn{2}{|c|}{ STEP }} \\
\hline LMNT & VAR & PARAM & MINIMUM & INITIAL & FINAL & MAXIMUM & & \\
\hline 5 & 1 & 10 & -0.322 & 0.328 & 0.3278000720 & 0.967 & $9.764 \mathrm{E}-15$ & \\
\hline 5 & 2 & 11 & -1.83 & -0.606 & -0.6064336572 & 0.609 & $1.729 \mathrm{E}-14$ & \\
\hline 5 & 3 & 12 & 0.00 & 0.724 & 0.7244206873 & 1.45 & $1.081 \mathrm{E}-14$ & \\
\hline \multirow{2}{*}{\multicolumn{5}{|c|}{ STATUS OF CONSTRAINTS }} & & & & \\
\hline & & & & & WEIGHT & CHED & $\mathrm{KI} 2$ & * Parameter (s) \\
\hline
\end{tabular}




$\begin{array}{llllllllll}10 & 1 & 1 & 994 & 0.0000 & 0.3000 & 1.2911894 \mathrm{E}-13 & 2.0915 \mathrm{E}-03 & * & 0 \\ 10 & 1 & 2 & 994 & 0.0000 & 0.3000 & 1.1151080 \mathrm{E}-12 & 0.1560 & * & 0 \\ 10 & 1 & 3 & 994 & 0.0000 & 1.000 & 8.6353147 \mathrm{E}-12 & 0.8419 & * & 0\end{array}$

\section{B.3 Zgoubi files, ring closed orbit search using FIT}

\section{zgoubi.dat including FIT input :}

Data generated by searchco

' OBJET'

.001 .001 .001 .0010 .01 .001

$\begin{array}{llllll}-6.431975 \mathrm{E}-01 & -4.982951 \mathrm{E}-01 & 0.0 \mathrm{E}+00 & 0.0 \mathrm{E}+00 & 0.0 \mathrm{E}+00 & 1.00000000 \mathrm{E}+00\end{array}$

'MARKER' MARK BSUPERA

'MULTIPOL' SBEN AIBE

0 . Dip

$\begin{array}{lllllllllllllll}200.6554 & 10.00 & 0.11712499 & 0.04848519 & -0.00050563 & 0.0 & 0.0 & 0.0 & 0.0 & 0.0 & 0.0 & 0.0\end{array}$

$0.0 .10 .00 \quad 4.0 \quad 0.800 \quad 0.00 \quad 0.00 \quad 0.00 \quad 0.00 \quad 0.0 .0 .0$.

$\begin{array}{llllllll}4 & .1455 & 2.2670 & -.6395 & 1.1558 & 0 . & 0 . & 0 .\end{array}$

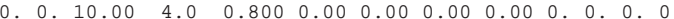

$\begin{array}{lllllllll}4 & .1455 & 2.2670 & -.6395 & 1.1558 & 0 . & 0 . & 0\end{array}$

$0.0,0.0,0.0,0.0,0.0$.

$\# 20|20| 20$ Dip AiBF

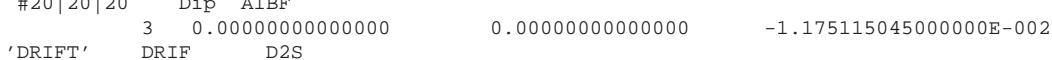

60.9515

..................

$\cdots \cdots \cdots \cdots \cdots$

'MULTIPOL' HKIC SML20

0 .kicker

$\begin{array}{lllllllllllllll}0.2413 \mathrm{E}+03 & 10.00 & 0.000000 \mathrm{E}+00 & 0.000000 & 0.0 & 0.0 & 0.0 & 0.0 & 0.0 & 0.0 & 0.0 & 0.0\end{array}$

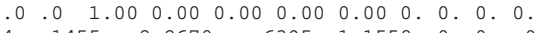

$\begin{array}{lllllllll}4 & .1455 & 2.2670 & -.6395 & 1.1558 & 0 . & 0 . & 0 .\end{array}$

$\begin{array}{lllllllllllll}.0 & .0 & 1.00 & 0.00 & 0.00 & 0.00 & 0.00 & 0.0 .0 .0 .0 .0 & 0.0 .0\end{array}$

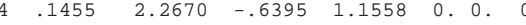

0.000000000 0.0.0.0.0.0.0.0.0.

$\# 20|20| 20$ Kick

1 0. 0.0 . 0 .

'DRIFT' DRIF

31.7339
'MARKER' MARK

111

'FAISCEAU'

'FIT'

$\begin{array}{llll}3 & 30 & 0 & 3\end{array}$

$\begin{array}{llll}1 & 31 & 0 & 3\end{array}$

$\begin{array}{llll}1 & 35 & 0 & .1\end{array}$

$\begin{array}{llllllll}3.1 & 1 & 2 & 978 & 0 . & 1 & 0 & \text { x_co }\end{array}$

$\begin{array}{lllllllll}3.1 & 1 & 3 & 978 & 0 . & 1 & 0 & x p+c 0\end{array}$

3.1 697880707.610 co length

\section{zgoubi.res including FIT output :}

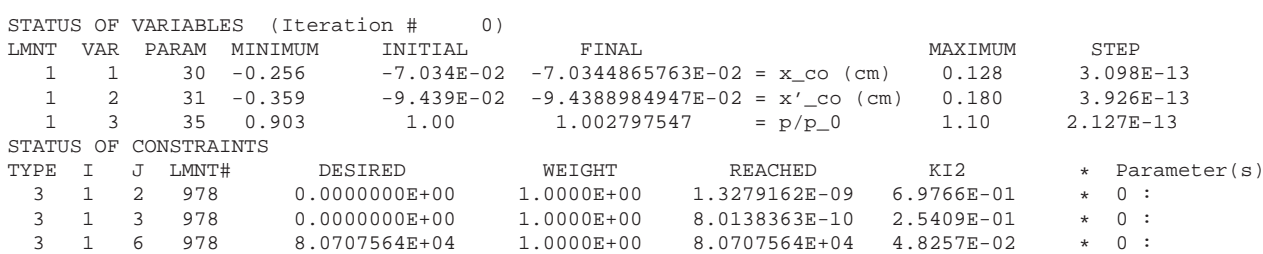

Path length of particle 1 : $80707.564 \mathrm{~cm}$

$\begin{array}{rrrrrr}\text { TRANSFER MATRIX } & \text { ORDRE } & 1 \text { (MKSA units) } & & \\ 0.732535 & -16.3490 & 0.00000 & 0.00000 & 0.00000 & 2.92379 \\ 0.145067 & -1.87262 & 0.00000 & 0.00000 & 0.00000 & 0.118147 \\ 0.00000 & 0.00000 & -0.909176 & -11.6183 & 0.00000 & 0.00000 \\ 0.00000 & 0.00000 & 0.175032 & 1.13689 & 0.00000 & 0.00000 \\ -0.338693 & 3.56095 & 0.00000 & 0.00000 & 1.00000 & 11.6934 \\ 0.00000 & 0.00000 & 0.00000 & 0.00000 & 0.00000 & 1.00000\end{array}$

$\begin{array}{rccccc}\text { First order sympletic conditions (expected values }=0 \text { ) } & : \\ -4.5006 \mathrm{E}-05 & -5.9300 \mathrm{E}-05 & 0.000 & 0.000 & 0.000 & 0.000 \\ \text { Beam matrix } & \text { (beta/-alpha/-alpha/gamma) } & \text { and periodic } & \text { dispersion } & \text { (MKSA units) } \\ 19.898601 & 1.585380 & 0.000000 & 0.000000 & 0.000000 & 2.059607 \\ 1.585380 & 0.176567 & 0.000000 & 0.000000 & 0.000000 & 0.145139 \\ 0.000000 & 0.000000 & 11.694319 & -1.029730 & 0.000000 & 0.000000 \\ 0.000000 & 0.000000 & -1.029730 & 0.176183 & 0.000000 & 0.000000 \\ 0.000000 & 0.000000 & 0.000000 & 0.000000 & 0.000000 & 0.000000 \\ 0.000000 & 0.000000 & 0.000000 & 0.000000 & 0.000000 & 0.000000\end{array}$

$\begin{array}{rr}\text { Betatron } & \text { tunes } \\ \text { NU_Y }=0.65346381 & \text { NU_Z }=0.76816038\end{array}$ 